\title{
Towards breeding \\ thrips-resistant \\ varieties in \\ Capsicum
}

Pauline van Haperen 


\section{Propositions}

1. In metabolomics, correlations divert scientists from finding causal mechanisms.

(this thesis)

2. Expression of resistance in one specific plant part confers a sufficient resistance level to the whole plant.

(this thesis)

3. The biggest challenge in life sciences is reproducibility.

4. Scientific journals should demand a layman's summary.

5. The biggest threat to academic freedom in the Netherlands is the current funding system.

6. Winning the battle to prevent food waste starts with a school trip to the farm.

Propositions belonging to the thesis entitled

Towards breeding thrips-resistant varieties in Capsicum

Pauline van Haperen

Wageningen, 24 March 2020 



\section{Towards breeding thrips- resistant varieties in Capsicum}

Pauline van Haperen 


\section{Thesis committee}

\section{Promotor}

Prof. Dr J.J.A. van Loon

Personal chair at the Laboratory of Entomology

Wageningen University \& Research

\section{Co-promotors}

Dr B.J. Vosman

Scientist, Plant Breeding

Wageningen University \& Research

Dr R.E. Voorrips

Scientist, Plant Breeding

Wageningen University \& Research

\section{Other members}

Prof. Dr M.E. Schranz, Wageningen University \& Research

Dr H. Huits, Bejo Zaden B.V., Warmenhuizen

Dr W.J. de Kogel, Wageningen University \& Research

Dr J.L. Peters, Radboud University, Nijmegen

This research was conducted under the auspices of the Graduate School of Experimental Plant Sciences (EPS) 


\section{Towards breeding thrips- resistant varieties in Capsicum}

\section{Pauline van Haperen}

\section{Thesis}

submitted in fulfilment of the requirements for the degree of doctor at Wageningen University

by the authority of the Rector Magnificus,

$$
\text { Prof. Dr A.P.J. Mol, }
$$

in the presence of the

Thesis Committee appointed by the Academic Board

to be defended in public

on Tuesday 24 March 2020

at 4 p.m. in the Aula. 
Pauline van Haperen

Towards breeding thrips-resistant varieties in Capsicum, 160 pages

PhD thesis, Wageningen University, Wageningen, the Netherlands (2020) With references, with summaries in English and Dutch

ISBN: 978-94-6395-285-9

DOI: https://doi.org/10.18174/512122 


\section{Table of Contents}

$\begin{array}{lll}\text { Chapter } 1 & \text { General introduction } & 7\end{array}$

Chapter 2 The effect of plant development on thrips resistance in Capsicum

Chapter 3 Fine mapping of a QTL for thrips resistance in Capsicum

Chapter 4 The role of diterpene glycosides in the resistance mechanism controlled by a thrips resistance QTL in Capsicum

Chapter 5 The effect of a thrips resistance QTL in different genetic backgrounds

Chapter 6 The contribution of two QTLs to thrips resistance in Capsicum

Chapter 7 General discussion

Summary

Samenvatting

References

Acknowledgements

About the author 


\section{CHAPTER 1}

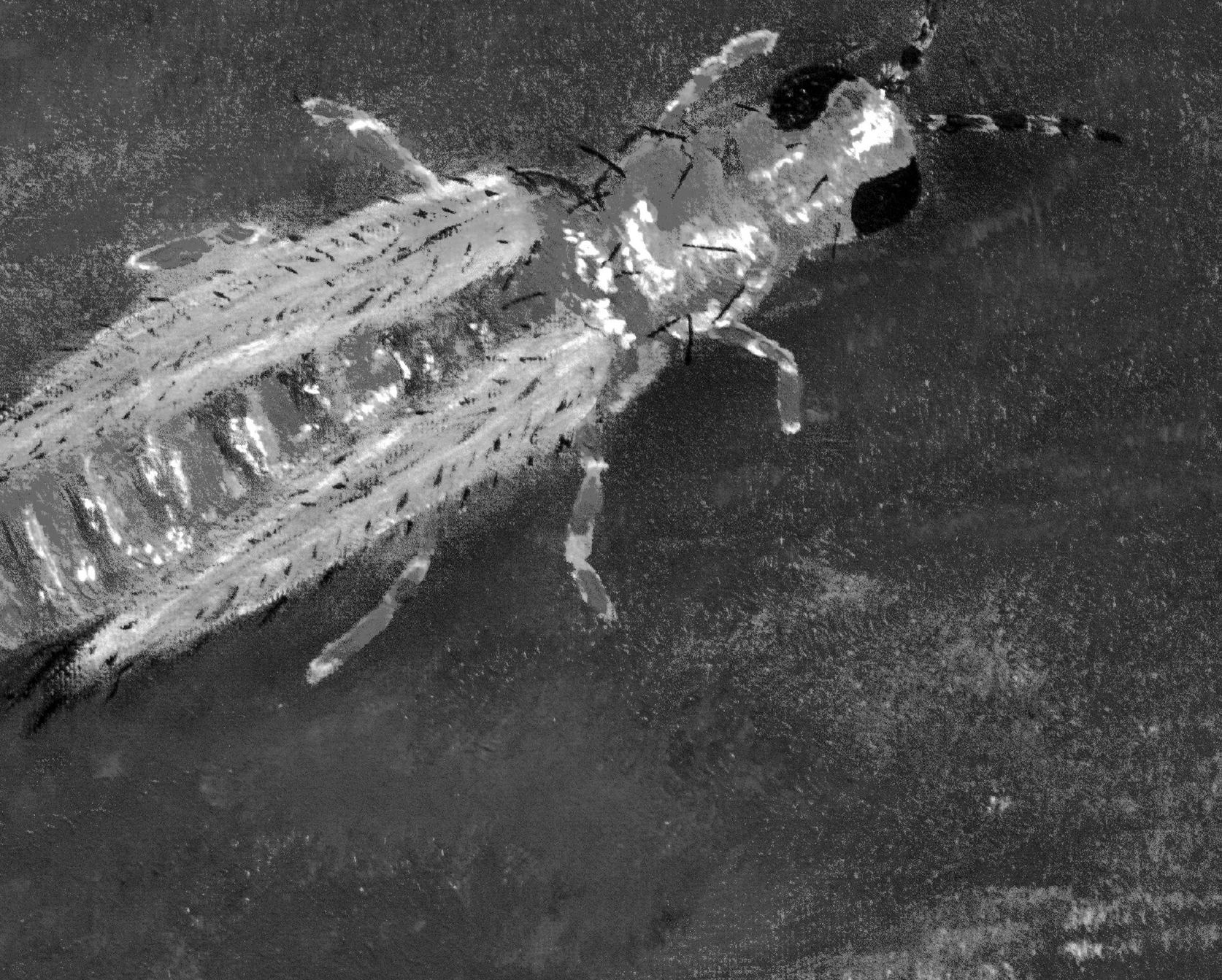


General Introduction 


\section{Crop domestication and its possible threats in pepper}

Domestication of crops has been a key event in human history (Diamond, 2002). Crop domestication is defined as the process of artificial selection of plants to increase their suitability to meet human needs (Doebley et al., 2006; Chen et al., 2015). The selection of plants based on desired traits such as size, shape, quality, nutritious value, and adaptation to cultivation has led to the development of new crops that are phenotypically different from their wild ancestors (Meyer et al., 2012). However, this selection may also have led to unintended loss of traits that are important in plant defence (Herms and Mattson, 1992; Rosenthal and Dirzo, 1997). It has been shown that chemical defence against herbivorous pests has become reduced under crop domestication, which resulted in increased damage to crops by herbivores (Rosenthal and Dirzo, 1997; Chen et al., 2015). For example, a wild relative of maize, teosinte, has higher levels of resistance to a number of pests compared to cultivated maize (de Lange et al., 2014). Another example of reduced resistance in cultivars compared to wild relatives was found in Brassica. It has been shown that generalist and specialist herbivores perform significantly worse on wild Brassica species compared to cultivated Brassica oleracea (Gols et al., 2008).

Similarly, pepper growers face several threats as many pepper (Capsicum annuum L.) cultivars are susceptible to diseases. Therefore, breeders would greatly benefit from host plant resistance mechanisms against these threats. For instance, different Capsicum accessions showed host plant resistance to pepper viruses such as pepper huasteco begomovirus (PHV), pepper golden mosaic virus (PepGMV), tomato spotted wilt virus (TSWV), pepper leaf curl virus (PepLCV) and cucumber mosaic virus (CMV) (Boiteux et al., 1993; Grube et al., 2000; Hernández-Verdugo et al., 2001; Anaya-López et al., 2003; Kumar et al., 2006); others showed resistance to other pathogens causing for instance bacterial wilt, anthracnose, Phytophthora root rot and to root knot nematodes (Kimble and Grogan, 1960; Peter et al., 1984; Matsunaga and Monma, 1999; Voorrips et al., 2004; Lebeau et al., 2011; Heitor Valim et al., 2013; Gonçalves et al., 2014).

Next to viruses and pathogens, pepper growers also face another important threat: pest insects. Insects not only damage pepper plants through feeding, but some species may transmit plant viruses and spread diseases from plant to plant (Kenyon et al., 2014). For instance, some whitefly species such as Bemisia tabaci (Gennadius) and Trialeurodes vaporariorum (Westwood) can act as vectors of over 200 plant viruses, mainly begomoviruses (Morales and Jones, 2004; Morales, 2006; Hidayat and Rahmayani, 2007); some aphids such as Myzus persicae (Sulzer) and Aphis gossypii (Glover) vector viruses such as pepper mottle virus, pepper severe mosaic virus, pepper yellow mosaic virus and potato virus $\mathrm{Y}(\mathrm{Ng}$ 
and Perry, 2004; Kenyon et al., 2014); and some thrips species such as Frankliniella occidentalis (Pergande) and Thrips tabaci (Lindeman) may transmit numerous viruses such as tospoviruses (Jones, 2005). Therefore, many accessions have been screened for resistance against these pest insects, and some accessions with intermediate or high level of resistance have been identified (Láska et al., 1982; Fery and Schalk, 1991; Maris et al., 2003; Frantz et al., 2004; Firdaus et al., 2011; Maharijaya et al., 2011; Sun et al., 2018).

Host plant resistance mechanisms against insects and other arthropods can work in different ways. In general, three categories have been distinguished: antibiosis, antixenosis and tolerance (Painter, 1951; Smith, 2005). In antibiotic resistance mechanisms, the mechanism has a negative effect on the insect's biology, for instance through affecting the insect's survival, fecundity or other life history parameters. In antixenotic resistance mechanisms, previously referred to as nonpreference (Painter, 1951; Kogan and Ortman, 1978), the insect's behaviour is affected, for instance due to production of feeding or oviposition deterrents. When a plant tolerates the insect, it can cope with the insect and recover from insect damage (Koch et al., 2016). Both antibiotic and antixenotic resistance mechanisms can be useful when targeting insects that transmit viruses; in contrast, tolerance may not be useful as insect feeding continues and the virus can still be spread from plant to plant.

\section{Thrips, a major pest insect in Capsicum annuum}

One of the major pest insect groups in Capsicum cultivation is thrips (Thysanoptera) (Siemonsma and Kasem, 1994). Thrips are small piercing-sucking insects of which many species can feed on multiple plants (Lewis, 1973; Mound and Walker, 1982; Kirk, 2002). Female adults lay eggs inside the host plant (Figure 1). First instar larvae hatch from these eggs after a few days (Mollema et al., 1993). First instar larvae develop into second instar larvae. After the second larval instar, pupation occurs. Depending on the species, thrips may have one prepupal stage and one or two pupal stages, before they reach the adult stage (Lewis, 1973). The egg to adult development time depends on environmental factors such as temperature, photoperiod and host plant (Lublinkhof and Foster, 1977; Brødsgaard, 1994; McDonald et al., 1998; Ishida et al., 2003). Female thrips can reproduce both sexually and asexually, depending on the species (Lewis, 1973; Jenser and Szénási, 2004; Wang et al., 2014; Ding et al., 2018).

The most common thrips species that causes damage to Capsicum production in the field and in the greenhouse worldwide is $F$. occidentalis, also known as the western flower thrips (Tommasini and Maini, 1995). Therefore, many studies on thrips in Capsicum focus on this species (Fery and Schalk, 1991; Hansen et al., 
2003; Kindt et al., 2003; Maris et al., 2003; Maharijaya et al., 2011; Maharijaya et al., 2012; Maharijaya et al., 2015; Macel et al., 2019; Maharijaya et al., 2019; Sarde et al., 2019; Visschers et al., 2019a; Visschers et al., 2019b). Other thrips species cause damage to pepper plants as well. For instance, T. tabaci, onion thrips, used to be the most prevalent thrips species in Europe before the introduction of $F$. occidentalis, and can still infest greenhouse crops such as Capsicum (Van Lenteren and Loomans, 1999). Scirtothrips dorsalis (Hood), chili thrips, and Thrips palmi (Karny), melon thrips, are major thrips pests in pepper cultivation in tropical and subtropical regions (Amin, 1979; Nuessly and Nagata, 1995; Krishna Kumar et al., 1996; Cannon et al., 2007; Weintraub, 2007). Another example of a thrips species causing damage to pepper is Thrips parvispinus Karny, one of the major thrips pests in south east Asia (Johari et al., 2014). In total, at least 16 thrips species have been reported to feed on Capsicum (Capinera, 2001).

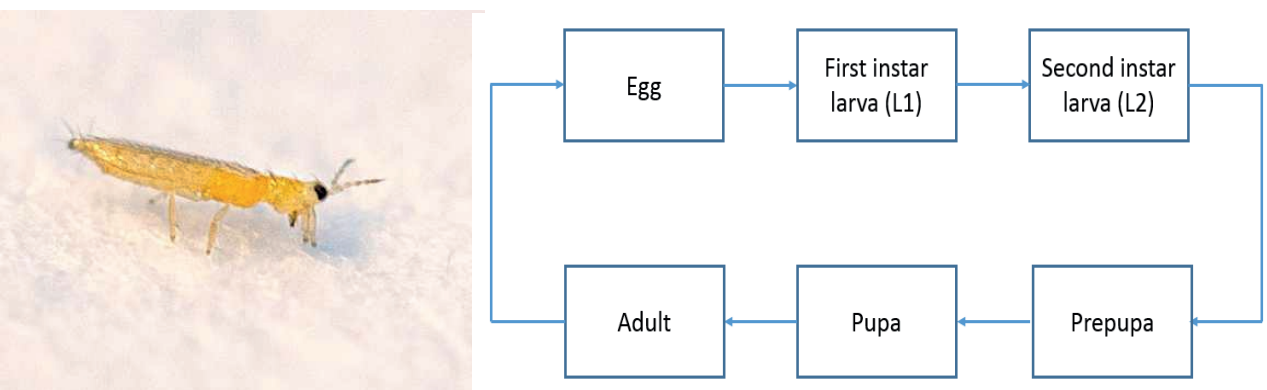

Figure 1: Adult stage of Frankliniella occidentalis (Pergande) and its life cycle. Eggs are laid inside the host plant, after which the larvae hatch and feed on the host plant. The pupal stages occur either on the host plant or in the soil, after which the adult emerges.

\section{Direct damage}

Thrips cause direct damage to plants through piercing epidermal plant cells with their stylet-shaped mouth parts and sucking out the cell content of flowers, leaves and fruits, leaving silvering scars generally referred to as silvering damage (Chisholm and Lewis, 1984; Rosenheim et al., 1990). Some thrips species, including $F$. occidentalis and $T$. tabaci, also feed on pollen, which can lead to an increase in thrips fecundity and a reduction in development time (Kirk, 1985; Murai, 2000; Hulshof and Vanninen, 2002). Thrips feeding leads to plant organ deformation, altered carbon allocation, reduced photosynthesis capacity, reduced plant growth, reduced fruit set, and thus reduced marketable yield (Welter et al., 1990; Tommasini and Maini, 1995; Shipp et al., 1998a). 


\section{Indirect damage}

Thrips can also cause damage to their hosts indirectly through the transmission of plant viruses. Up to now, 14 thrips species have been described that can transmit tospoviruses (Jones, 2005; Riley et al., 2011). The most important vector of tospoviruses is considered to be $F$. occidentalis. It can transmit several species of tospoviruses such as chrysanthemum stem necrosis virus (CSNV), groundnut ringspot virus (GRSV), impatiens necrotic spot virus (INSV), tomato chlorotic spot virus (TCSV) and tomato spotted wilt virus (TSWV) (reviewed by Jones (2005)). First instar larvae and young second instar larvae can acquire tospoviruses when feeding on infected plants (Moritz et al., 2004). The virus replicates in the thrips, mainly in its midgut, salivary glands and surrounding muscle cells and ligaments (reviewed by Ullman et al. (2002)). When the infected thrips larvae develop into second instar larvae that are about to pupate or have become adults, thrips can reintroduce tospoviruses to plants through their saliva during probing (Whitfield et al., 2005). Adult thrips remain viruliferous for life (Jones, 2005).

TSWV is the most widespread of the tospoviruses and the biggest viral threat to Capsicum vectored by thrips (Kenyon et al., 2014). The most common symptoms of TSWV infection in pepper include spotting, bronzing, necrosis of leaves and ringspots on the fruits (Mandal et al., 2006; Salamon and Szabó, 2016). Plants infected in a young stage may show necrosis on petioles and stems as well. Their fruits are deformed and show concentric pale or yellow rings that may become necrotic. Plants infected at later stages may develop symptoms in only part of the plant, whereas other parts remain healthy, due to the inability of the virus to move into mature parts of the plant (Mandal et al., 2007). It is shown that female $F$. occidentalis are more attracted to pepper plants infected with TSWV, both for feeding and oviposition, leading to increased numbers of viruliferous thrips (Maris et al., 2004).

\section{Economic damage threshold in Capsicum}

Several studies have aimed to determine the economic threshold, i.e. the thrips density at which the costs of controlling the population size exceeds the costs of crop damage. Park et al. (2007) showed that the fruit damage was directly correlated to $F$. occidentalis density in pepper, and estimated the economic threshold to range between 0.7 to 2.1 adults or nymphs per flower, or 2.3 to 5.7 adults per four-day sticky card count. Shipp et al. (1998b) estimated the economic threshold to range from 10 to 26 adults per sticky trap per day. These values are highly dependent on market prices and seasonal stages of pepper production, and only consider the direct, and not indirect thrips damage. These economic thresholds show that the thrips population should be controlled in order to prevent large economic damage. 


\section{Control of thrips}

Nowadays growers use protective measures to prevent that thrips densities exceed the economic threshold. These measures consist of mechanical, cultural, physical, chemical and biological control (Mouden et al., 2017). Some examples of protective measures applied in pepper are the use of ultraviolet light reflective mulches, insecticides, natural predators such as Amblyseius swirskii (AthiasHenriot), Orius insidiosus (Say) or Orius laevigatus (Fieber) (Tommasini and Maini, 2002; Reitz et al., 2003; Tommasini et al., 2004; Broughton and Herron, 2009; Calvo et al., 2012). However, these measures only limit thrips population development partially, as they are difficult to control due to their high reproductive rate, short life cycle and cryptic behaviour (Cloyd, 2009). In addition, the use of insecticides against thrips is not desired, not only because of the public demand to avoid chemical control, but also due to the chances that thrips populations develop resistance to insecticides (Jensen, 2000). Therefore, host plant resistance to thrips is a highly desired trait in Capsicum.

\section{Screening for host plant resistance to thrips in Capsicum}

Over the years, different screening methods for host plant resistance to thrips in pepper have been developed and used to identify thrips-resistant accessions. In general, these methods can be divided into two categories: "choice" and "nochoice". In choice experiments, parameters such as thrips damage, reproduction and preference are determined in a setting where thrips can freely move between plants, leaves or leaf discs. The resistance mechanisms determined in choice experiments might affect insect behaviour or preference (antixenosis) rather than life history parameters (antibiosis) and deter thrips in the presence of an accession more suitable or attractive to thrips. Accessions that seem resistant in choice experiments should always be validated in no-choice experiments, as the availability of more suitable plants for feeding and reproduction might lead to identifying false positive resistant accessions. In no-choice experiments, parameters such as thrips damage, reproduction and survival are conducted on thrips that are restricted to plants, leaves or leaf discs of a single accession. Resistance mechanisms identified in no-choice settings are especially interesting to breeders, as thrips also do not have a choice between accessions in commonly applied greenhouse cultivation.

Thrips-resistant pepper accessions have been identified based on level of feeding damage, host preference, host suitability for reproduction, larval survival, pupal survival and oviposition rate, either on intact plants, detached leaves or leaf discs (Fery and Schalk, 1991; Maris et al., 2007; Maharijaya et al., 2011, 2012 and 2015; Visschers et al., 2019a; 2019b). Previously, thrips damage was mainly assessed by eye. Recently, new techniques have become available to 
automatically quantify thrips damage on leaf discs, leading to objective, standardized and high-throughput methods for screening for thrips resistance (Visschers et al., 2018). Other methods to study thrips feeding behaviour on resistant and susceptible accessions are the electrical penetration graph technique (EPG-DC system) and automated video tracking of thrips feeding behaviour. With EPG, thrips are attached to a wire in an electrical circuit consisting of thrips, plant and soil. When thrips penetrate, salivate or ingest plant tissue, the electrical circuit is closed and three different basic EPG waveforms are recorded that are each specific to one of the feeding behaviours (Harrewijn et al., 1996, Kindt et al., 2003). With video tracking, thrips movement is recorded in dual-choice arenas (Thoen et al., 2016). Preference was deduced from the total time spent on either one of the arenas, and movement or non-movements was translated into thrips feeding behaviour parameters (i.e. searching, pausing and feeding).

Several studies using different screening methods have led to the identification of sources of resistance to thrips in Capsicum (Fery and Schalk, 1991; Maris et al., 2003; Maharijaya et al., 2011; Maharijaya et al., 2012; Maharijaya et al., 2015; Macel et al., 2019; Maharijaya et al., 2019; Visschers et al., 2019a; Visschers et al., 2019b). These studies made a start towards understanding the possible resistance mechanisms. Maharijaya et al. (2012) showed that larval development is inhibited in three resistant Capsicum accessions. Macel et al. (2019) and Maharijaya et al. (2019) showed a correlation between thrips resistance and diterpene glycosides as well as some other metabolites in Capsicum.

\section{Mapping thrips resistance in Capsicum}

When thrips-resistant accessions are identified, mapping populations can be made to determine which genetic loci contribute to thrips resistance. To our knowledge, thrips resistance in Capsicum has only been mapped in two independent mapping studies. Both studies used the same resistant accession, i.e. CGN16975, as a resistance donor. In the mapping study of Linders et al. (2015), a quantitative trait locus (QTL) for resistance to $F$. occidentalis was mapped to chromosome 5 in a F2 population that resulted from a cross between CGN16975 and an unknown susceptible Capsicum annuum accession. Silvering damage in a choice setting was used as resistance parameter. In the mapping study of Maharijaya et al. (2015), a QTL for resistance to $F$. occidentalis was identified in a population that resulted from a cross between the susceptible accession CGN17219 and the resistant accession CGN16975 (Maharijaya et al., 2015). Larval survival, pupal survival and larval feeding damage in a no-choice setting were used as resistance parameters. 


\section{Scope and thesis outline}

This study is a continuation of the study of Maharijaya (2013), in which he identified several Capsicum accessions resistant to $F$. occidentalis and $T$. parvispinus (Maharijaya et al., 2011). The first characterization of the resistance mechanism in three resistant Capsicum accession showed that larval development was inhibited on the youngest fully opened leaves from resistant accessions at a plant age of 12 weeks (Maharijaya et al., 2012). One of the resistant accessions, C. annuum CGN16975, was crossed with a susceptible C. chinense CGN17219 to create a mapping population, in which one major resistance QTL that explained about $50 \%$ of the variance was mapped to chromosome 6 (Maharijaya et al., 2015). This finding contrasted with a different study of Linders et al. (2015) in which a resistance QTL was mapped to chromosome 5 in a mapping population that has the same resistant parent (CGN16975). It was hypothesized that the resistant parent has two major resistance factors, but only one of those resistance factors segregated in each mapping population, which would indicate that both factors are necessary for resistance. Furthermore, a metabolomics approach was used to detect metabolite QTLs (mQTLs), and six mQTLs co-located with the resistance QTL on chromosome 6 (Maharijaya et al., 2019). The causal gene(s) in the QTL region that confer thrips resistance remained unknown.

In my study, I aim to answer research questions that resulted from the work of Maharijaya (2013). An overview of the content of each chapter can be found below:

In Chapter 2, the effect of plant development on thrips resistance in Capsicum was studied. Maharijaya et al. (2012) characterized thrips resistance in leaves of one leaf age, i.e. the youngest fully opened leaves, from plants from the same age, i.e. 12 weeks. Here I aim to determine whether plant age and leaf age affect thrips resistance. Resistance to thrips was determined in plants from nine accessions with different levels of thrips resistance and of three different ages. Also, larval development was determined on leaves of one susceptible and one resistant accession of different age classes. These findings can be implemented in integrated pest management strategies.

In Chapter 3, the QTL on chromosome 6 identified by Maharijaya et al. (2015) was further fine mapped and validated. Expression of genes in the fine mapped QTL region was quantified to determine which candidate genes are most likely to play a role in thrips resistance.

In Chapter 4, I aim to determine whether metabolites play a role in thrips resistance conferred by the resistance QTL. Maharijaya et al. (2019) identified six mQTLs that co-located with the previously identified resistance QTL, two of which 
correlated with high concentrations of diterpene glycosides. In this chapter, levels of metabolites are compared between plants with the resistance allele or the susceptibility allele in homozygous state. In this way I can investigate whether known pathways or metabolites are controlled by the resistance QTL on chromosome 6.

In Chapter 5, the effect of the QTL on chromosome 6 in four different genetic Capsicum annuum backgrounds was studied. This QTL was previously only studied in the genetic background of the mapping population (Maharijaya et al., 2015), thus it is not known whether the QTL would inhibit larval development in different genetic backgrounds. Larval development of $F$. occidentalis and $T$. tabaci was studied in plants with the resistance or the susceptibility allele in homozygous state within the same genetic background. The potential of this QTL in breeding thrips-resistant varieties is discussed.

In Chapter 6, I determined the contribution of the QTLs on chromosome 5 and 6 to thrips resistance. The line from Linders et al. (2015) was characterized in larval development and silvering damage assays in a no-choice setting. Possible explanations for the contradicting findings in the mapping study of Linders et al. (2015) and Maharijaya et al. (2015) are discussed.

In Chapter 7, a general discussion of the most important findings of this thesis is provided. I focus on the potential application of the resistance QTL in breeding for thrips resistance and on future perspectives for thrips resistance research. 


\section{CHAPTER 2}
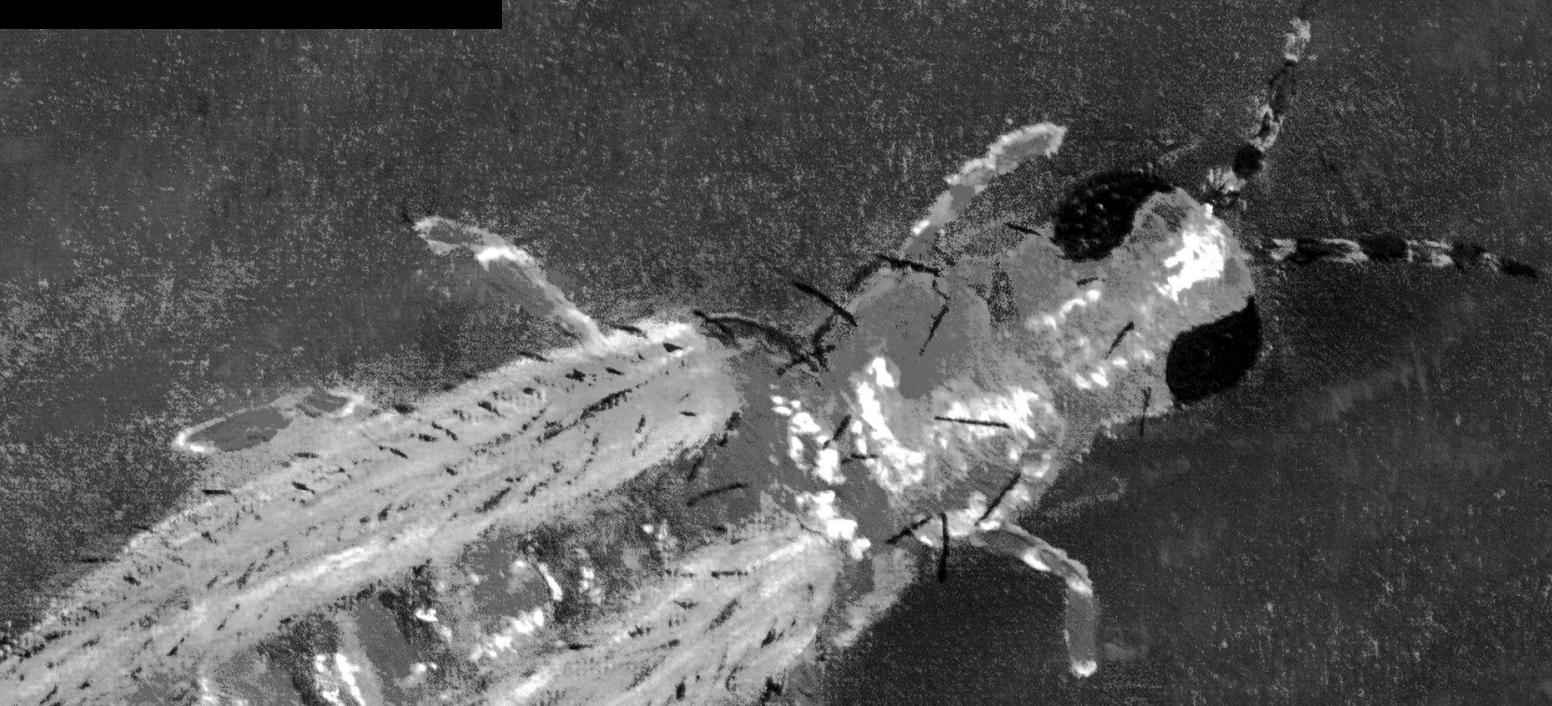

: nd 40

(l)

1.4.

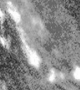

$=-x^{2}$
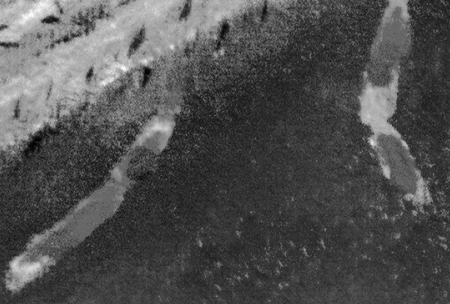


\section{The effect of plant development on thrips resistance in Capsicum}

Pauline van Haperen, Roeland E. Voorrips, Joop J.A. van Loon, Ben Vosman 


\begin{abstract}
Western flower thrips (Frankliniella occidentalis (Pergande)) is a worldwide pest insect that causes damage in pepper cultivation, so growers would benefit from host plant resistance. The objectives of this study were (1) to evaluate the effect of plant age on thrips resistance using nine Capsicum accessions with different levels of thrips resistance at three different plant ages, and (2) to study the effect of leaf age on thrips resistance in a resistant and a susceptible pepper accession. The fraction of first instar larvae that did not develop into second instar was used as a measure for thrips resistance. Our results show that plants start to develop thrips resistance when they are between four and eight weeks old. This transition was clearest on the resistant accession CGN16975, on which about 50\% of the L1 larvae developed into the next stage on four-week-old plants, whereas none of them developed beyond the L1 stage on eight or twelve-week-old plants. Furthermore, it is shown that youngest fully opened leaves of the resistant accession CGN16975 are significantly more resistant to thrips than older leaves; $89 \%$ of the L1 larvae did not develop into the next stage on the youngest leaves, whereas $57 \%$ did not develop beyond the L1 stage on the oldest leaves. Young leaves of the susceptible accession CGN17219 are more susceptible than older leaves; 9 versus $52 \%$ of the L1 larvae did not develop into the next stage on young and old leaves respectively. These findings can be used to improve Integrated Pest Management strategies.
\end{abstract}




\section{Introduction}

Thrips is a worldwide pest insect in agricultural and horticultural crops (Kirk and Terry, 2003). The most important thrips species in Capsicum in Europe is western flower thrips (Frankliniella occidentalis (Pergande)), causing constraints to pepper production both in the field and in the greenhouse (Siemonsma and Kasem, 1994; Tommasini and Maini, 1995). Thrips can damage plants both directly and indirectly. Direct damage is caused by feeding on the leaves, flowers and fruits, resulting in their deformation, reduced plant growth, altered carbon allocation, and thus reduced yield (Welter et al., 1990; Shipp et al., 1998a). Very characteristic is the "silvering damage" which is caused by thrips piercing the plant cells with their stylet-shaped mouth and sucking out the cell content, leaving silver leaf scars (Chisholm and Lewis, 1984). Indirect damage is caused by the viruses they transmit, of which tomato spotted wilt virus (TSWV) is the most important one in pepper (German et al., 1992).

It is difficult to control thrips because of their high reproductive rate, short life cycle and cryptic behaviour (Cloyd, 2009). Monitoring the thrips population and limiting its growth to prevent an outbreak is therefore important. This can at least partly be accomplished by using cultural, mechanical, physical and biological control (Mouden et al., 2017). In addition to these protective measures, it is highly desirable to identify plants that have a natural source of resistance against thrips, either constitutive or induced. Host plant resistance can be found in several plant species and in several forms (Smith, 2005; Broekgaarden et al., 2011) for example, by the presence of a modified epicuticular wax layer on leaves of cabbage (Voorrips et al., 2008; Znidarcic et al., 2008); or by the production of specific compounds such as acylsugars that have a negative effect on insects (Mirnezhad et al., 2010; Glas et al., 2012).

Host plant resistance to insects has been found in a large number of crops. In some cases the resistance is only expressed at specific plant developmental stages. For instance, the epicuticular lipids that play a role in resistance against herbivorous insects can vary with plant part, plant age and environmental condition (Eigenbrode and Espelie, 1995). Another example is the increasing resistance in a Brassica oleracea cultivar against cabbage whitefly when these plants grow older (Broekgaarden et al., 2012). Similar findings have been reported in resistance of Solanum lycopersicum against tomato leafminer (Leite et al., 2001). In addition, it has been shown that resistance levels may vary in different parts of the plant (De Kogel et al., 1997b; Leiss et al., 2009a). 
Host plant resistance in pepper against thrips may also be affected by plant development. Maharijaya et al. (2011) screened several pepper accessions when the plants were twelve weeks old. Larval development and mortality was determined on the youngest fully opened leaves only (Maharijaya et al., 2012). Therefore, it is not known whether the host resistance against thrips that was identified by Maharijaya et al. (2011) changes during plant development, and whether the resistance varies between plants of different age and leaves of different age.

The aims of this study are to determine whether or not the resistance in pepper against thrips changes during plant development, and whether or not the level of resistance varies between leaves of the pepper plant. Based on previous studies on the effect of plant development on insect resistance (De Kogel et al., 1997b; Leite et al., 2001; Leiss et al., 2009a; Broekgaarden et al., 2012), we hypothesized that the level of resistance to thrips in young leaves increases with plant age, and that thrips resistance decreases with leaf age.

\section{Material and methods}

\section{Plant material and growing conditions}

To study whether plant age affects thrips resistance, we used the same nine Capsicum accessions as Maharijaya et al. (2012), which were classified as susceptible, intermediate resistant and fully resistant to thrips, based on the damage to the youngest fully opened leaves by female adults of $F$. occidentalis and development of first instar larvae (L1). Seeds were obtained from the Centre of Genetic Resources, The Netherlands. Three groups of four plants of each accession were sown with a four week interval in potting compost in a greenhouse of Unifarm, Wageningen University and Research, Wageningen, The Netherlands. Plants were grown at $25^{\circ} \mathrm{C}$, with a photoperiod of L16:D8, and $70 \% \mathrm{RH}$. No insecticides were applied. Thrips were controlled biologically using the predatory hemipteran Orius laevigatus (Fieber) (Entocare C.V., Wageningen, The Netherlands). Plants were watered three times per week, two times per week nutrients were added. The three youngest fully opened leaves of each plant were evaluated for thrips resistance 12 weeks after the first group of plants was sown, so at plant ages of 12,8 and 4 weeks, respectively. Plants were not yet flowering when the youngest fully opened leaves were collected.

To study whether different levels of thrips resistance are found in leaves of different age, we selected one resistant (CGN16975) and one susceptible (CGN17219) accession. Five plants of each accession were sown and grown in the same conditions as the plants of the plant age experiment. At a plant age of 12 weeks we collected leaves of five age classes (1-5, corresponding to a leaf age of 
about 0, 2, 4, 6 and 8 weeks, respectively) for use in a detached leaf assay for thrips resistance (Additional material 1, Electronic Supplementary Material 1). Three leaves of each leaf age class were collected per plant. These leaves were the three youngest fully opened leaves of each plant at a plant age of $12,10,8$, 6 and 4 week. Plants of CGN16975 and CGN17219 had 4 and 6 real leaves respectively at a plant age of 4 weeks. Plants of both accessions showed the same number of leaves at a plant age of 6 weeks or older. The plants did not flower until after the leaves were evaluated for thrips resistance at the plant age of 12 weeks.

Plants from accessions CGN16975 and CGN17219 were used to determine the thrips damage in a no-choice whole plant damage assay. Five plants of each accession were sown and grown in the same conditions as the plants of the plant age experiment. Four weeks after sowing, the plants were enclosed in thrips-proof sleeves to prevent thrips from escaping from the plant.

Three plants from accession CGN16975 were used to study oviposition by female thrips. From each plant the main stem was removed at a plant age of 20 weeks, and the largest side branch that emerged from the base of the main stem was enclosed in a thrips-proof sleeve to prevent thrips from escaping. Each side branch contained at least 40 old and 40 young leaves. The plants were flowering at the start of the oviposition experiment.

\section{Thrips rearing}

A population of Frankliniella occidentalis (acquired from Greenhouse Horticulture of Wageningen University and Research, Bleiswijk, The Netherlands) was reared on Phaseolus vulgaris beans in glass rearing pots covered with thrips-proof gauze in a growth cabinet at $25^{\circ} \mathrm{C}, \mathrm{L16}$ :D8, $70 \%$ relative humidity. Female adults were allowed to lay eggs on snack cucumbers for 24 hours, after which the thrips were brushed off and the cucumbers were kept in the growth cabinet at $25^{\circ} \mathrm{C}$. After 4 days, new synchronized first instar larvae (L1) emerged (Mollema et al., 1993).

\section{Detached leaf assay}

Each detached leaf, selected as discussed in the Plant material section, was placed with the petiole in a droplet of $1.5 \%$ water agar in a Petri dish (BD Falcon, tightfit lid $50 \times 9 \mathrm{~mm}$ ). Five synchronized L1 larvae were placed on each detached leaf. The Petri dish was firmly closed to prevent the larvae from escaping. The Petri dishes were incubated at $25^{\circ} \mathrm{C}$ in a growth cabinet. The developmental stage of each thrips was determined at day 3,5 and 7 post infestation. The fraction of larvae that did not develop from the first into the second larval stage (from L1 to L2) was used as a measure for thrips resistance. Larval development was more 
consistent as a measure for thrips resistance than larval survival. In some replicates survival was affected by the condensation of water causing droplets on the lids of the firmly closed Petri dishes after 5 to 7 days, that caused some larvae to drown. Since under optimal conditions, first instar larvae develop into second instar larvae in 1-2 days (Lublinkhof and Foster, 1977), larval development was not affected by the formation of water droplets, and was therefore selected as resistance criterion.

\section{Whole plant damage assay}

For the whole plant damage assay, female adults were carefully collected by using an aspirator and anesthetized with carbon dioxide $\left(\mathrm{CO}_{2}\right)$. When seven weeks old plants were infested each with 20 female adult thrips. To ensure high thrips pressure, the infestation was repeated one week later. The five most damaged leaves of each plant were scored for silvering damage, caused by thrips feeding, when the plants were ten weeks old. The plants did not yet flower when they were scored. The damage scale is shown in Table 1.

Table 1: Evaluation scale for a whole plant thrips damage assay. The five most affected leaves of each plant were evaluated. The damage scale is adapted from Linders et al. (2015)

\begin{tabular}{|l|l|l|}
\hline $\begin{array}{l}\text { Leaf } \\
\text { damage } \\
\text { scale }\end{array}$ & Description & $\begin{array}{l}\text { \% of } \\
\text { silvering } \\
\text { damage }\end{array}$ \\
\hline 1 & No damage & 0 \\
\hline 2 & Tiny silvering spots & $<0.1$ \\
\hline 3 & Some small spots near the mid vein or edge of the leaf & $0.1-1$ \\
\hline 4 & $\begin{array}{l}\text { Moderate number of spots near the mid vein or edge of the leaf, } \\
\text { or some small spots more regularly distributed over the entire } \\
\text { leaf }\end{array}$ & $1-2$ \\
\hline 5 & $\begin{array}{l}\text { Moderate number of spots, more regularly distributed over the } \\
\text { entire leaf }\end{array}$ & $3-5$ \\
\hline 6 & Intermediate between 5 and 7 & $6-10$ \\
\hline 7 & Many silvering spots over the entire leaf & $11-20$ \\
\hline 8 & Intermediate between 7 and 9 & $21-40$ \\
\hline 9 & Very heavy silvering, large leaf surface area damaged & $>40$ \\
\hline
\end{tabular}

\section{Oviposition}

For the oviposition assay, female adults were carefully collected by using an aspirator and anesthetized with $\mathrm{CO}_{2}$. Each selected side branch was infested with 75 adult females. After three days, the 40 oldest and 40 youngest leaves of each side branch were removed and each leaf was placed in a separate Petri dish as described above. All adult thrips were removed. The leaf area was determined by analysing pictures of the leaves in Image] $1.51 \mathrm{f}$ (Schneider et al., 2012). The Petri dishes were incubated at $25^{\circ} \mathrm{C}$ in a growth cabinet. Emerged larvae were counted 
and removed on 6,8 and 10 days after the initial infestation. As the average development time from egg to the first instar is three to four days at $25^{\circ} \mathrm{C}$, we assumed that all viable eggs were hatched within ten days after infestation (McDonald et al., 1998; Zhang et al., 2007). The average leaf area of young leaves and the average leaf area of old leaves respectively were used to determine the number of larvae per $\mathrm{cm}^{2}$ of leaf area per leaf age category.

\section{Statistical analysis}

The fractions of larvae arrested at the L1 stage were transformed as $y=\arcsin (\sqrt{ } x)$. Analysis of variance (ANOVA) was carried out to test for significant differences in larval development between plants and leaves of different ages. For the plant and leaf age experiment, a two-way ANOVA was carried out to determine the effects of age, accession and the interaction between age and accession respectively. When significant F-values $(P<0.05)$ were obtained, all mean values in the plant and leaf age experiment respectively were compared using Fisher's Protected least-significant-difference (LSD) test. For the whole plant damage assay, a MannWhitney $U$ test was used to determine whether the differences between damage score of the most affected leaves of the two tested accessions were significant. For the oviposition assay, a Wilcoxon matched-pairs signed-ranks test was used to determine whether the differences between emerged larvae on old and young leaves per $\mathrm{cm}^{2}$ of leaf area were significant. These statistical analyses were conducted using Genstat $18^{\text {th }}$ edition (VSN International, 2015).

\section{Results}

\section{Plant age experiment}

The two-way ANOVA showed a significant interaction between accession and plant age in their effect on the fraction of larvae arrested at the first instar stage $(P<$ 0.001 , Table 2). A significant effect of plant age on larval development was found in six of the nine tested accessions (Table 3). In five of these accessions (PRI1996112, PRI1996236, CGN17042, CGN20503 and CGN16975), a significantly lower average fraction of L1 was found after seven days when the plants were four weeks old, compared to the plants from the same accession that were eight or twelve weeks old. In one of the accessions, the most susceptible accession CGN17219, a significantly higher average fraction of L1 was found after seven days in four-week-old plants compared to eight- and twelve-week-old plants. We did not find a significant difference between eight- and twelve-weekold plants among all tested accessions. 
Table 2: Two-way ANOVA results for the effects of plant age and Capsicum accession on larval development of Frankliniella occidentalis

\begin{tabular}{lrrrrr}
\hline Source of variation & d.f. & Sum of squares & Mean square & F-ratio & P \\
\hline Plant age & 2 & 3.90 & 1.95 & 27.87 & $<.001$ \\
Accession & 8 & 28.86 & 3.61 & 51.62 & $<.001$ \\
Plant age.Accession & 16 & 5.40 & 0.34 & 4.83 & $<.001$ \\
Residual & 183 & 12.79 & 0.07 & & \\
Total & 209 & 50.95 & & & \\
\hline
\end{tabular}

Table 3: Larval development of Frankliniella occidentalis in nine Capsicum accessions at different plant ages. At the indicated plant age, the three youngest fully opened leaves of four plants of the same accession were infested with five L1 larvae in a detached leaf assay and seven days later the fraction that remained in L1 stage was determined (average fraction of L1)

\begin{tabular}{|c|c|c|c|c|c|}
\hline \multirow[b]{2}{*}{$\begin{array}{l}\text { Accession } \\
\text { code }\end{array}$} & \multirow[b]{2}{*}{ Accession } & \multirow[b]{2}{*}{$\begin{array}{l}\text { Level of } \\
\text { resistance }\end{array}$} & \multicolumn{3}{|c|}{ Average Fraction of L1 (7dpi) per plant age $\mathrm{e}^{\mathrm{b}}$} \\
\hline & & & 4 weeks & 8 weeks & 12 weeks \\
\hline CGN17219 & $\begin{array}{l}\text { C. chinense } \\
\text { no } 4661\end{array}$ & $S(2.6)$ & 0.06 (bc) & $0.00(a)$ & $0.00(a)$ \\
\hline PRI1996108 & $\begin{array}{l}\text { C. chinense } \\
\text { PI281428 }\end{array}$ & $\mathrm{S}(2.9)$ & $0.11(\mathrm{~cd})$ & 0.17 (cde) & $0.13(\mathrm{~cd})$ \\
\hline PRI1996112 & $\begin{array}{l}\text { C. chinense } \\
\text { PI315023 }\end{array}$ & $\mathrm{S}(2.8)$ & $0.02(a b)$ & 0.37 (defg) & 0.29 (def) \\
\hline PRI2004001 & $\begin{array}{l}\text { C. annuum } \\
\text { Bruinsma Wonder }\end{array}$ & $M(1.8)$ & 0.43 (cdefgh) & 0.18 (cde) & 0.15 (cde) \\
\hline CGN22817 & $\begin{array}{l}\text { C. frutescens } \\
\text { Lombok }\end{array}$ & $M(1.4)$ & 0.24 (def) & 0.40 (fgh) & 0.30 (efgh) \\
\hline PRI1996236 & $\begin{array}{l}\text { C. annuum } \\
\text { Laris }\end{array}$ & $M(1.1)$ & 0.17 (cde) & $0.45(h)$ & $0.43(\mathrm{gh})$ \\
\hline CGN17042 & $\begin{array}{l}\text { C. baccatum } \\
\text { no1553 }\end{array}$ & $\mathrm{R}(0.8)$ & $0.30(\mathrm{efgh})$ & $0.77(\mathrm{i})$ & $0.88(\mathrm{ij})$ \\
\hline CGN20503 & $\begin{array}{l}\text { C. annuum } \\
\text { Bisbas }\end{array}$ & $\mathrm{R}(0.6)$ & 0.32 (efgh) & $0.88(\mathrm{ij})$ & $0.93(j)$ \\
\hline CGN16975 & $\begin{array}{l}\text { C annuum } \\
\text { AC1979 }\end{array}$ & $\mathrm{R}(0.3)$ & $0.47(h)$ & $1.00(\mathrm{k})$ & $1.00(\mathrm{k})$ \\
\hline
\end{tabular}

a Based on the results of previous screening by Maharijaya et al. (2011), in which damage scores were given ( $0=$ no injury, $3=$ severe injury, $S=$ susceptible, $M=$ intermediate resistant, $\mathrm{R}=$ resistant)

${ }^{b}$ All means followed by the same letter are not significantly different (Fisher's protected LSD test, $\mathrm{P}>0.05$ ) 


\section{Leaf age experiment}

To study the possible effect of leaf age on resistance we selected the most susceptible and the most resistant accession from the plant age experiment, CGN17219 and CGN16975 respectively. The two-way ANOVA showed a significant interaction between accession and leaf age on larval development $(P<0.001$, Table 4). Leaf age affected larval development in the resistant and susceptible accession in opposite ways (Table 5). In the resistant accession, we found that on the youngest fully opened leaf the fraction of L1 larvae was significantly higher than on leaves of the other ages. In the susceptible accession, we found that larval development significantly increases as leaf age decreases.

Furthermore, it was shown that the differences in larval development when comparing leaves of the same age between the susceptible and resistant accession were significant when the leaf was six weeks or younger (Table 5). The largest contrast between the accessions was found in the youngest leaves. When leaves were eight weeks old, no significant differences between the resistant and susceptible accession were found.

Table 4: Two-way ANOVA results for the effects of leaf age and Capsicum accession on larval development of Frankliniella occidentalis

\begin{tabular}{lrrrrr}
\hline Source of variation & d.f. & Sum of squares & Mean square & F-ratio & P \\
\hline Leaf age & 4 & 0.84 & 0.21 & 4.54 & 0.002 \\
Accession & 1 & 8.49 & 8.49 & 183.12 & $<.001$ \\
Leaf age.Accession & 4 & 5.56 & 1.39 & 29.99 & $<.001$ \\
Residual & 134 & 6.21 & 0.05 & & \\
Total & 143 & 21.11 & & & \\
\hline
\end{tabular}

Table 5: Larval development of Frankliniella occidentalis in a resistant (CGN16975) and a susceptible (CGN17219) pepper accession on leaves of different ages. The leaves were tested for larval development when the plant was 12 weeks old in a detached leaf assay, by infesting each leaf with five L1 larvae and determining the fraction of larvae that remained in the $L 1$ stage at seven days after infestation (average fraction of $L 1$ ). Leaves of ages 1-5 were about $0,2,4,6$ and 8 weeks old at time of testing, respectively

\begin{tabular}{lll}
\hline & \multicolumn{2}{l}{ Average fraction of L1 } \\
\cline { 2 - 3 } Leaf age & CGN16975 & CGN17219 \\
\hline 1 & 0.89 (a) & 0.09 (e) \\
2 & 0.69 (b) & 0.15 (e) \\
3 & 0.58 (bc) & 0.31 (d) \\
4 & 0.61 (b) & 0.46 (c) \\
5 & 0.57 (bc) & 0.52 (bc) \\
\hline
\end{tabular}

${ }^{a}$ All means followed by the same letter are not significantly different (Fisher's protected LSD test, $\mathrm{P}>0.05$ ) 


\section{Whole plant damage assay}

In addition to the leaf age experiment, the most resistant accession CGN16975 and most susceptible accession CGN17219 were also used in a no-choice whole plant damage assay when plants were ten weeks old. The damage was determined by evaluating the five most damaged leaves in five plants of these two accessions. Most thrips damage was found on the oldest leaves in both accessions. Thrips damage score was significantly higher for the susceptible accession CGN17219 than for the resistant accession CGN16975 (Figure 1).

\section{Oviposition assay}

Plants of the resistant accession CGN16975 were used in an oviposition assay to study whether there is a difference in oviposition on young and old leaves. The total number of emerged larvae per $\mathrm{cm}^{2}$ of leaf area at ten days after infestation was determined. A significantly higher number of larvae per $\mathrm{cm}^{2}$ emerged from young leaves compared to old leaves (Figure 2).

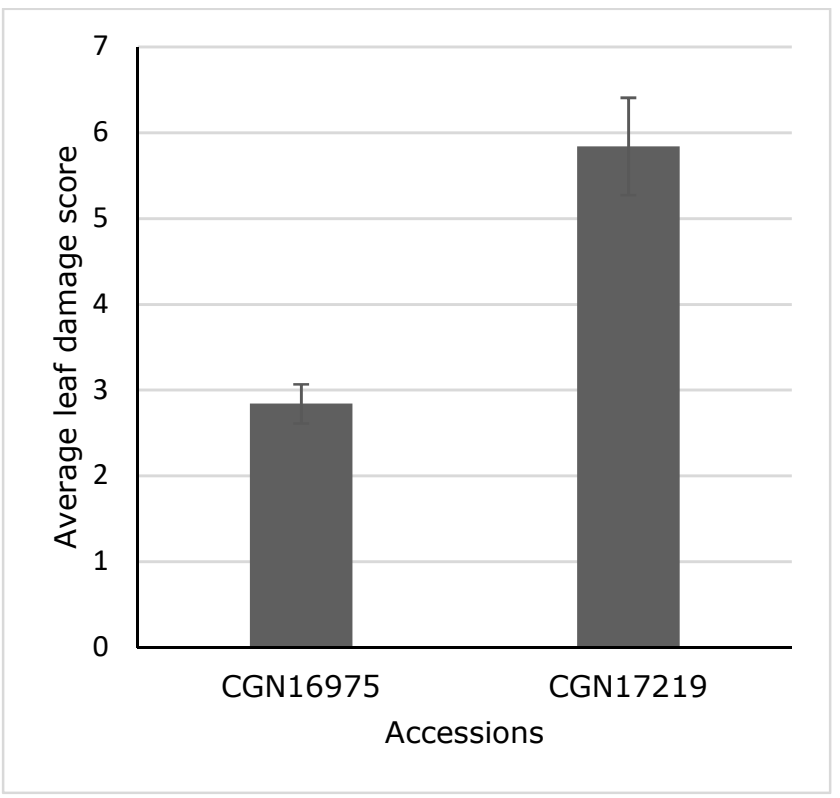

Figure 1: Average leaf damage score of the five most affected leaves of five whole plants of the resistant accession CGN16975 and the susceptible accession CGN17219. Each plant was infested twice with twenty female adult Frankliniella occidentalis when the plants were seven and eight weeks old. The damage of each of the five most affected leaves per plant was scored according to the scale in Table 1 when the plants were 10 weeks old. The error bars indicate the standard error. The average leaf damage score for CGN16975 was significantly lower than the score for CGN17219 $(P<0.001)$ 


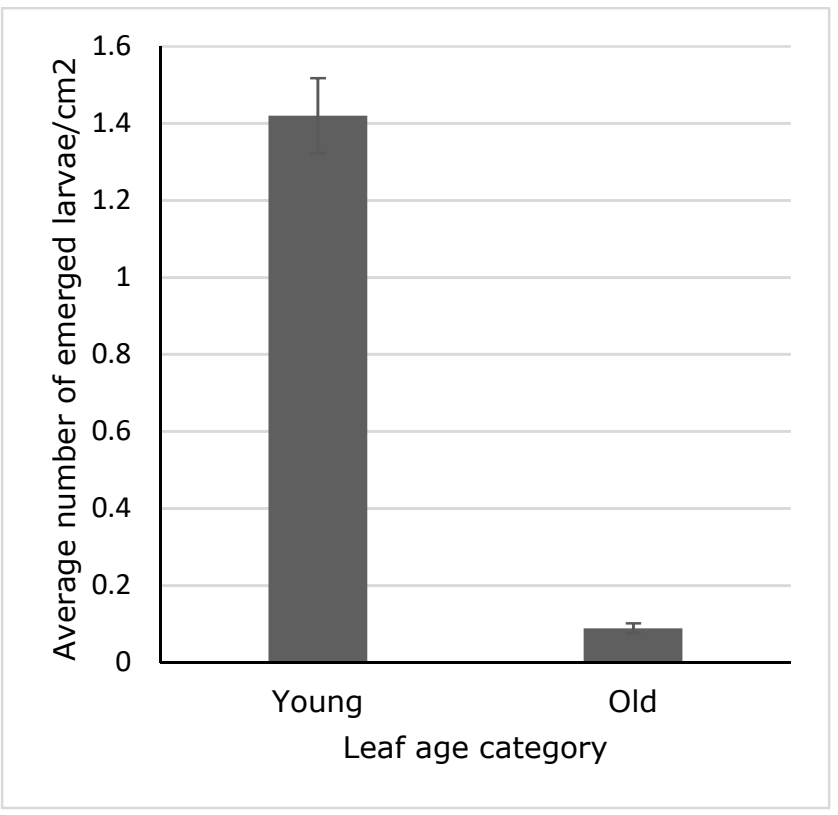

Figure 2: Average number of emerged larvae per $\mathrm{cm}^{2}$ of leaf area in young and old leaves of three lower side branches of three plants of CGN16975 at $10 \mathrm{dpi}$. Each side branch was infested with 75 female adult Frankliniella occidentalis for three days. Up to ten days after infestation, the emerged larvae on the 40 youngest and 40 oldest leaves of each side branch were counted. The error bars indicate the standard error of the mean. The average number of emerged larvae per $\mathrm{cm}^{2}$ was significantly lower in old leaves compared to young leaves $(P<0.001)$

\section{Discussion}

\section{Plant age affects thrips resistance}

We showed that on four-week-old plants of all accessions L1 larvae can develop to the L2 stage, although there are significant differences between the accessions. Between plant ages of four and eight weeks we observed an increased resistance in five of the accessions (Table 3). This transition was most marked on CGN16975, the most resistant accession included in this study, on which about half of the L1 larvae were able to develop into the next stage on four weeks old plants, whereas on eight or twelve-week-old plants they did not develop at all beyond the L1 stage. This finding suggests that thrips resistance increases with plant age. Differences in insect resistance between plants of different ages is also found in other crops. For instance, Bemisia tabaci Gennadius resistance in Solanum pennellii L. is higher in twenty-week-old plants than in six-week-old plants (Van den Oever-Van den Elsen et al., 2016), and twelve-week-old Brassica oleracea L. plants have higher resistance to the whitefly Aleyrodes proletella L. than six-week-old plants (Broekgaarden et al., 2012). Whether the resistance that is developing with plant 
age in the resistant Capsicum accessions is constitutive (also present in the absence of the thrips) or induced by thrips feeding remains an open question. Further research may shed light on this. We also observed a clear interaction between plant age and accession. In contrast to resistant accessions, susceptible accessions do not show a significant increase in thrips resistance when plant age increases.

\section{Leaf age affects thrips resistance}

We observed significant differences in thrips resistance between young and older leaves of the resistant accession CGN16975 and the susceptible accession CGN17219. When comparing the leaves between the two accessions, no significant difference in thrips resistance was found between the oldest leaves of the resistant and susceptible accession; on these leaves almost half of the L1 larvae develop into the next stages on both accessions. In the resistant accession CGN16975 the youngest fully opened leaves show the highest thrips resistance. A similar observation was made in Senecio, in which Leiss et al (2009a) found a significant positive correlation between leaf age and silvering damage in a choice assay, suggesting a higher thrips resistance in younger leaves. Another example of higher insect resistance in younger leaves is described by Alvarez et al. (2014), who found that green peach aphid Myzus persicae Sulzer resistance in younger leaves of potato was higher than in older leaves. In contrast, the susceptible pepper accession CGN17219 shows a higher larval development on younger leaves compared to older leaves. The contrasting effect on larval development on the resistant and susceptible accession can explain the observed interaction between leaf age and accession in their effect on larval development. The higher larval development on younger leaves of the susceptible accession may be explained by higher nutrition and water contents of young compared to old or senescent leaves (Mattson, 1980; Boege and Marquis, 2005). A similar observation was made by De Kogel et al. (1997b), who observed a higher thrips reproduction on apical leaves compared to middle or basal leaves, both in a choice and non-choice study on three partially resistant and one susceptible cucumber accession. These studies used different methods and criteria than we did, so a direct comparison with the outcomes of these studies is not possible. However, these results suggests that in general young leaves are better suited for thrips performance than older leaves, but that resistance, if present, is expressed most strongly in the younger leaves, resulting in a trade-off. The differences in thrips resistance between young and old leaves in both the resistant and the susceptible accession might be related to the levels of defence compounds. In general, young leaves often contain higher concentrations of defence compounds than older leaves (McCall and Fordyce, 2010). 
The results from the leaf age experiment agree with our observations in the whole plant damage assay. The average damage score of all leaves evaluated for thrips damage per accession shows that the resistant CGN16975 plants received a significantly lower damage score than the susceptible CGN17219. This difference is found despite the lower level of thrips resistance in the older leaves compared to the youngest leaves within plants of the resistant accession, indicating that the thrips resistance in the youngest leaves does reduce whole plant thrips damage. In this no-choice experimental set-up, the thrips caused silvering damage to the older leaves in the resistant accession as well, which raises the question whether the thrips oviposit on the older leaves, enabling them to reproduce on this accession. In potato, Alvarez et al. (2014) showed that young leaves were resistant to the green peach aphid, but that the insects survive and reproduce on older leaves. The oviposition assay showed that female adult thrips oviposit on both old and young leaves. Despite the resistance against thrips in the young leaves, females laid a significantly higher number of eggs per $\mathrm{cm}^{2}$ on young leaves compared to old leaves, suggesting that the resistance in the young leaves is useful, as newly emerged larvae on young leaves are directly exposed to host plant resistance. Badenes-Perez et al. (2014) described a similar observation in plants of the genus Barbarea: Plutella xylostella L. laid more eggs on young leaves compared to older leaves although the abundance of two plant secondary metabolites that were suggested to play a role in plant defence and feeding deterrence was higher in young leaves.

Based on these findings, we formulated the following hypothesis. In general, leaf nutritional quality decreases as leaves grow older (Mattson, 1980; Boege and Marquis, 2005). Because of the better leaf quality, thrips larvae perform better on young leaves than on old leaves of susceptible accessions. In resistant accessions, the resistance is highly expressed only in young leaves, thus preventing the thrips to develop there. During aging of the leaves the resistance factor decays, resulting in similar levels of susceptibility in the older leaves of both resistant and susceptible accessions. As female adult thrips strongly prefer to oviposit on young leaves of a resistant plant, this suggests they are unable to detect or react to (a) resistance factor(s) that affect first instar larvae negatively. Therefore a high resistance level in young leaves is useful for the plant despite the susceptibility of older leaves.

\section{Implications for plant resistance breeding and Integrated Pest Management}

This study provides information to improve the methods to determine host resistance against thrips in Capsicum. The plant age experiment shows that the level of thrips resistance can be determined when plants are eight weeks old 
instead of twelve weeks old, because no significant difference in thrips resistance in eight and twelve week old plants was found. The leaf age experiment shows that the largest contrast in thrips resistance between a resistant and a susceptible accession was found in the youngest leaves, indicating that a comparison between these leaves could provide most information on the mechanism of the resistance based e.g. on gene and protein expression and metabolite profile. The whole plant damage assay shows that, although the older leaves of the resistant accession do not provide resistance against thrips, the resistance in the younger leaves does affect thrips damage, probably because oviposition predominantly takes place on these young leaves as shown by the oviposition assay. Therefore, this study shows that CGN16975 is a potential donor for host plant resistance against thrips.

The outcome of this study also provides information for pest management and crop protection. Considering that female adults can lay four to five eggs per day in an optimal environment in the absence of pollen (Van Rijn et al., 1995), the number of thrips can still increase rapidly and can subsequently cause large damage to the plant as almost half of the $L 1$ can develop into $L 2$ in young resistant plants. Therefore, it is important to protect plants until thrips resistance is fully expressed. It is recommended to use protection measures in combination with host plant resistance to limit the growth and spread of the thrips population. Extra protection can be provided for instance by applying UV-reflective mulch (Reitz et al., 2003) or by using water or sticky traps with attractive volatiles (Teulon et al., 1993; Koschier et al., 2000; Tang et al., 2016), leading to a decrease in number of $F$. occidentalis adults. Moreover, using a natural predator such as Orius insidiosus in an early plant stage can lead to a much greater decrease in number of $F$. occidentalis, thus keeping the thrips population below the economic threshold (Ramachandran et al., 2001).

\section{Acknowledgements}

We thank Betty Henken and Mengjing Sun for assistance with carrying out the detached leaf assays. This research was financially supported by a grant (TKI 1409-045) from the Ministry of Economic Affairs of The Netherlands and the breeding companies Nunhems Netherlands B.V. and Bejo Zaden B.V.

\section{Supplementary data}

Supplementary data Additional material 1 can be accessed through the following link: https://static-content.springer.com/esm/art\%3A10.1007\%2Fs11829-0189645-6/MediaObjects/11829 20189645 MOESM1 ESM.pdf 



\section{CHAPTER 3}

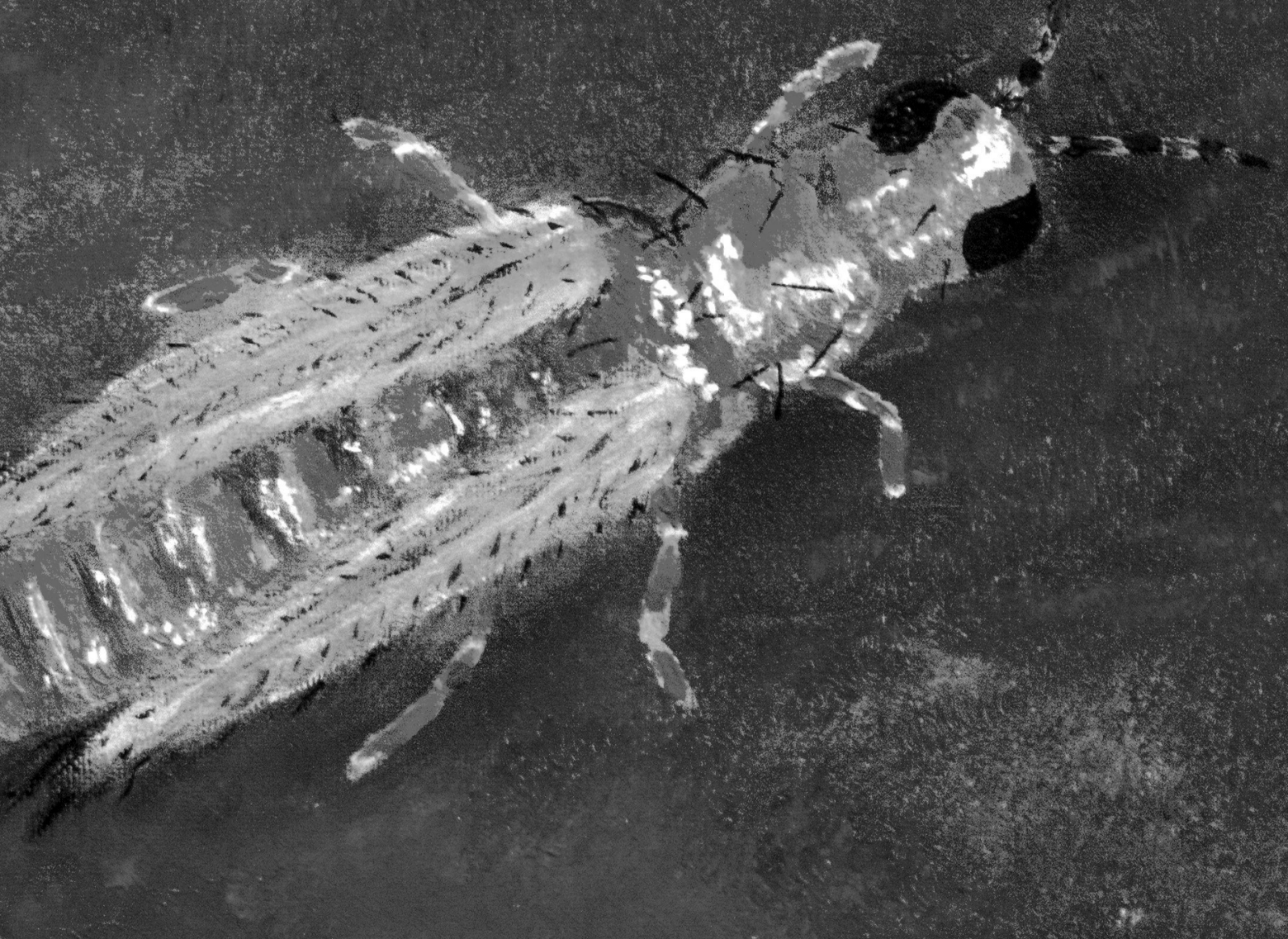




\section{Fine mapping of a QTL for thrips resistance in Capsicum}

Pauline van Haperen, Roeland E. Voorrips, Martijn van Kaauwen, Joop J.A. van Loon, Ben Vosman 


\section{Abstract}

Thrips resistance is an important trait for pepper/paprika growers. These insects can cause extensive damage to fruits, flowers and leaves, both on the field and greenhouse grown plants worldwide. In this study, we fine mapped a previously defined thrips resistance QTL on chromosome 6, to identify candidate genes that play a role in the resistance mechanism, to a region of $0.4 \mathrm{Mbp}$ harbouring 15 genes. Three of these 15 candidate genes showed either differences in gene expression when comparing plants carrying the resistance allele in homozygous state to plants with the susceptibility allele in homozygous state for the QTL region upon thrips induction, or had a SNP that was predicted to lead to changes in protein structure. Therefore, these three genes, i.e. an acid phosphatase 1 (APS1), an organic cation/carnitine transporter 7 (OCT7) and an uncharacterized locus LOC107874801, are the most likely candidates for playing a role in thrips resistance, and are a first step in further elucidating the thrips resistance pathway in Capsicum. 


\section{Introduction}

Thrips are major pest insects in crops worldwide, both in the field and in the greenhouse (Kirk and Terry, 2003; Morse and Hoddle, 2006). Thrips can cause direct damage to the crops by feeding on fruits, flowers and leaves, leading to their deformation, reduced growth, altered carbon allocation, and thus reduced quality and yield (Welter et al., 1990; Shipp et al., 1998a). Thrips can also damage plants indirectly by transmitting viruses such as tomato spotted wilt virus (TSWV) (reviewed by Jones (2005)).

Growers use protective measures to prevent thrips damage by limiting population growth. These protective measures consist of cultural, mechanical, physical and biological control (Mouden et al., 2017a). Current protective measures only partially affect thrips population growth because of the high reproductive rate, the short life cycle and the cryptic behaviour of the thrips (Cloyd, 2009). Biological control employing predation by Orius spp. (Hemiptera: Anthocoridae) limits thrips population growth, but predator pressure should remain high, as thrips population growth will occur if the predator population is too small (Sanchez and Lacasa, 2002; Tommasini et al., 2004). Controlling thrips through spraying crops with insecticides is also not desired, as, next to the public demand to avoid using insecticides, thrips populations can develop resistance to insecticides by changing their behaviour or by evolving detoxification enzymes that render active compounds inactive (reviewed by Jensen (2000)). Therefore, natural host plant resistance against thrips is a highly desired trait.

Screening wild accessions for thrips resistance and subsequent quantitative trait loci (QTL) mapping of the resistance factor(s) are important first steps in narrowing down the number of candidate genes that play a role in thrips resistance, and in developing thrips-resistant crops. Previous studies showed QTLS involved in resistance to Thrips palmi Karny in common bean (Phaseolus vulgaris) (Frei et al., 2005) and to Megalurothrips sjostedti Trybom (Omo-Ikerodah, 2008; Sobda, 2017), Thrips tabaci Lindeman and Frankliniella schultzei Trybom (Muchero 2010) in cowpea (Vigna unguiculata). However, in all these cases further fine mapping of the QTLs is still needed to find the gene or genes that are involved in thrips resistance.

In pepper, Maharijaya et al. (2011) identified the wild Capsicum annuum accession CGN16975 as a potential source for thrips resistance. Further characterization of the resistance showed that the larval development is inhibited on young leaves (Maharijaya et al., 2012)(Chapter 2). QTL mapping was carried out in an F2 population derived from an interspecific cross between the resistant Capsicum annuum CGN16975 and the susceptible Capsicum chinense CGN17219, 
and resistance to Frankliniella occidentalis was determined (Maharijaya et al., 2015). A single QTL that explained around $50 \%$ of the genetic variation was identified on chromosome 6. The resistance parameters used in this QTL analysis, i.e. larval survival from the L1 to $L 2$ stage, larval survival from $L 2$ to pupal stage, and leaf damage in a no-choice assay, all co-localize near the same marker on chromosome 6. No additional QTLs were found. The gene or genes that confer thrips resistance in Capsicum remain unknown.

The aim of this study was to fine map the QTL on chromosome 6 in order to identify the gene or genes that may play a key role in thrips resistance in Capsicum. We used plants that originated from the F2 mapping population that was developed by Maharijaya et al. (2015). In addition, we used gene expression data to determine which genes in the fine mapped QTL region are most likely to play a role in thrips resistance.

\section{Material and Methods}

\section{Overview of experiments and plant material}

The plant material that was used for QTL validation and fine mapping of the QTL originated from the mapping population of Maharijaya et al. (2015) (Table 1). Eleven F2 plants that were heterozygous for the QTL region were selfed. Five of the resulting F3 lines were randomly selected to validate the QTL. For each line, larval development on plants with the resistance ("R") allele in homozygous state was compared to larval development on plants with the susceptibility (" $S$ ") allele in homozygous state. Then, F3 plants with a recombination in the QTL region were selected for fine mapping the resistance QTL. These F3 plants were phenotyped and subsequently selfed for one or two generations, in order to obtain F4 and F5 plants with the recombination in the new fine mapped region in homozygous state, or the "R" or " $\mathrm{S}$ " allele in homozygous state, to further fine map and validate the new QTL region. Selfings from CGN16975 (CGN16975A; first generation) and CGN17219 (CGN17219A; second generation) were included as resistant and susceptible references respectively. Seeds were sown in potting compost in a greenhouse of Unifarm (Wageningen University and Research, Wageningen, the Netherlands). Plants were grown with a photoperiod of L16:D8 and 70\% RH at $25^{\circ} \mathrm{C}$. No insecticides were applied. Thrips were controlled using the Orius laevigatus (Fieber) (Entocare C.V., Wageningen, the Netherlands). Plants were watered three times a week. Two times a week, nutrients were added.

Gene expression was studied in two groups of plants, one of plants with the "R" allele and one of plants with the " $\mathrm{S}$ " allele in homozygous state. A heterozygous F3 plant for the QTL region on chromosome 6 was selfed to obtain F4 plants with 
the " $\mathrm{R}$ " or " $\mathrm{S}$ " allele in homozygous state. Plants were sown and grown under the same conditions as described above.

Table 1: Plant lines used in validation and fine mapping of the QTL region on chromosome 6. CGN16975A and CGN17219A were included as resistant and susceptible references. Plants with the resistance allele ("R") or susceptibility allele ("S") in homozygous state were selected to validate the QTL region on chromosome 6. F3 plants with a recombination in the QTL region were selected for phenotyping to fine map the resistance QTL. F3 plants with a recombination in the new fine mapped region were then selfed for one or two generations, and F4 or F5 plants with the recombination in homozygous state were selected for phenotyping to further fine map the QTL and to validate the fine mapped QTL region.

\begin{tabular}{|l|l|l|}
\hline Plant line & QTL chromosome 6 & Experiment \\
\hline CGN16975A & R & QTL validation and fine mapping \\
\hline CGN17219A & S & QTL validation and fine mapping \\
\hline F3 lines 1-5 & R or S & QTL validation \\
\hline F3 lines 1-10 & Recombination & Fine mapping \\
\hline F4 lines 1-9 & $\begin{array}{l}\text { Recombination, R or S in } \\
\text { homozygous state }\end{array}$ & Fine mapping and validation \\
\hline F5 lines 1-4 & $\begin{array}{l}\text { Recombination, R or S in } \\
\text { homozygous state }\end{array}$ & Fine mapping and validation \\
\hline F4 line 10 & R or S & Gene expression \\
\hline
\end{tabular}

\section{DNA extraction}

Young leaves of each individual plant were collected for DNA extraction in tubes containing two $2-\mathrm{mm}$ stainless steel beads each and stored in a $-80^{\circ} \mathrm{C}$ freezer until use. Samples were ground with a TissueLyser II (Qiagen) at $25 \mathrm{~Hz}$ for 70 seconds. Microprep buffer was prepared as described by Fulton et al. (1995), and $500 \mu \mathrm{L}$ was added to each ground leaf sample. The samples were shaken until homogenized and incubated at $65^{\circ} \mathrm{C}$ for 30 minutes, after which they were cooled for 5 minutes in ice water and $500 \mu \mathrm{L}$ chloroform was added. After gently shaking the samples for 40 times, the samples were centrifuged at $3500 \mathrm{rpm}$ for 15 minutes. The supernatant was transferred to a new tube and 0.8 volume of Isopropanol was added. The samples were turned for 40 times and centrifuged at $3500 \mathrm{rpm}$ for 15 minutes. The supernatant was discarded. The pellet was washed with $175 \mu \mathrm{L} 70 \%$ ethanol and dried. The pellet was re-suspended in $100 \mu \mathrm{L} \mathrm{MQ}$ and stored in the fridge at $-20{ }^{\circ} \mathrm{C}$ until use.

\section{Molecular markers design}

Single Nucleotide Polymorphism (SNP) discovery was carried out on RNAseq data of the $\mathrm{F} 1$ that resulted from a cross between the parents of the mapping population, i.e. the resistant accession C. annuum CGN16795 and the susceptible accession C. chinense CGN17219. RNA extracted from the leaves of the F1 plant was sequenced by BGI Genomics. SOAPdenovo (Luo et al., 2012) was used to 
make the assembly. The reads were mapped to the assembly using Bowtie2 (Langmead and Salzberg, 2012). We used qualitySNPng to detect SNPs (Tang et al., 2006; Nijveen et al., 2013). Fifteen SNP markers (Table 2) were selected in the previously identified QTL region (Maharijaya et al., 2015). Forward and reverse primers were designed using Primer3Plus (Untergasser et al., 2007).

An additional marker for fine mapping of the resistance was designed based on the whole genome re-sequencing of the resistant accession Capsicum annuum CGN16975 (M13.4). DNA was extracted from CGN16975A and sent to Novogene Technology Co., Ltd (Hong Kong), for library preparation and sequencing. Reads were mapped to the Capsicum annuum Zunla-1 assembly (Qin et al., 2014) and the UCD10X assembly (Hulse-Kemp et al., 2018) using BWA-mem (Li, 2013). The Integrative Genomics Viewer (Robinson et al., 2011) was used to find SNPs and their flanking sequences. Primers were designed using Primer3Plus (Untergasser et al., 2007).

\section{Genotyping}

Plants that were used to validate the previously identified QTL region and to fine map the thrips resistance were genotyped by Dr. van Haeringen Laboratorium B.V. (VHL Genetics company, Wageningen, Netherlands) with two SNP-markers (M2 and M15, Table 2), flanking the 2-LOD interval of the QTL-region as determined by Maharijaya et al. (2015), using KASP assays (Semagn et al., 2014). F3 plants with a recombination between these flanking markers were genotyped with additional markers M6, M7, M8, M10, M12, M13, and M14, Table 2) in the QTL region using KASP assays (Bejo Zaden B.V., Warmenhuizen, the Netherlands; Table 2). Additional markers in the area of interest (M10.1, M10.2, M10.3, M10.4, M11 and M441) were tested with LightScanner ${ }^{\circledR}$ System (Idaho Technology Inc.) using the small amplicon approach (Liew et al., 2004). F4 and F5 plants that were used to further fine map and validate the new QTL region were genotyped using KASP assays for M10, M10.1, M10.2, M10.3, M10.4, M12, M13, M14 and M15, and LightScanner assays for M11 and M13.4. LightScanner assays for M11 and M15 were used to genotype and select plants for differential gene expression analysis.

\section{Thrips rearing and synchronization}

The population of Frankliniella occidentalis originated from Greenhouse Horticulture of Wageningen University and Research (Bleiswijk, the Netherlands). Frankliniella occidentalis was reared in a growth cabinet at $25^{\circ} \mathrm{C}, \mathrm{L} 16: \mathrm{D} 8,70 \%$ relative humidity, on Phaseolus vulgaris beans in glass rearing jars covered with thrips-proof gauze. The first instar larvae (L1) were synchronized by allowing female adult thrips to lay eggs on snack cucumbers. After 24 hours, the female 
adults were brushed off and the cucumber was kept in the growth cabinet at $25^{\circ} \mathrm{C}$. Synchronized L1s emerged after 4 days.

Table 2: SNP markers and their flanking sequences in the QTL region on chromosome 6 used for genotyping

\begin{tabular}{|c|c|}
\hline Marker ID & SNP flanking sequence \\
\hline M2 & $\begin{array}{l}\text { GTGAGGAAAAGAAAAGTTIGTCGTATGAGATTACCTTTACTAGTAAGAGCAGGGG } \\
\text { TGGAGTGGAGATGGT[A/G]GAGGGGGTTCAATCTGCATTTGGATCTATTGAGTGG } \\
\text { AGTGATGGGGTTCACAATGTGAGAAGTCCAATTG }\end{array}$ \\
\hline M6 & $\begin{array}{l}\text { CTCTTGTGTGCTITCTITCTCTCACTCACTITCTACCTGTAACTGCTCT[G/A]CCT } \\
\text { CCTCAAACAAAGAGAGTGATGCCTAGCTGCGGAGAGAATTGAAAAAA }\end{array}$ \\
\hline M7 & $\begin{array}{l}\text { GCATAATTCTCTCCACAAGCACCACACAGTGTCTCGCCATGCTCATCCTC[G/A]TC } \\
\text { CTGCTCATCCAAGCCCTCCTCTTCATCTTTAGGCTGCATCTTTGTAAG }\end{array}$ \\
\hline M8 & $\begin{array}{l}\text { TTTGTGAAGAAGCAACCGGTGACAGGATAAAAGTGGCCGGTGCAAACCACCGGTT } \\
\text { CGGTGCACCAACTCCATTACCGGCCACACTGAGAATTATCGTCTATCGAGC(GAG) } \\
\text { 5GAAGAAACTGTTGGTGCTTCAGTAGCTACCACTAAAATATCGGTTCTTCGTIT } \\
\text { TATTCCTTCGCACTTCTTCCTCACA }{ }^{1}\end{array}$ \\
\hline M10 & $\begin{array}{l}\text { TTTGTTGGAAGCTACCTTGGATATAAAAAACCAGCTATTGAAGACCCTGT[T/C]AA } \\
\text { GACGAATAAAA } \\
\text { TCCCTAGGCAAATACCAGAGCAGGCATGGTACATGAA }\end{array}$ \\
\hline M10.1 & $\begin{array}{l}\text { AGAAAATATCTGACTTGAATGTGGACCCTTACTTAGGTGTTGCAAATGGC[A/T]GC } \\
\text { ATGCCCAGTTCAAGCGTTCTTCCAGTCAAAAACAGCATCTTGCAAAT }\end{array}$ \\
\hline M10.2 & $\begin{array}{l}\text { GTTCCAGGGCAATTAGTTGGGAGATTGTCAAGAAAAACAACTCTITIT[A/G]GT } \\
\text { TAAGGAGTTGGTAATGGTACTGCTGGTGTTGTCTTCAGCAAAGAGCC }\end{array}$ \\
\hline M10.3 & $\begin{array}{l}\text { CTATTCTCCTTCTCATITGACTTGATTCTGGAAAGTGGATTCATTTGCTC[G/A]ATA } \\
\text { GCTGAACTTGAAGCAGATGGCTITATCACAGGCATGGAATAAGGTTG }\end{array}$ \\
\hline M10.4 & $\begin{array}{l}\text { GATCATGGTTCTACTGGTGGCCTATGGAATATCCACACTAACAATGCAGT[C/T]TC } \\
\text { ATCCGGAGAATCTGCAATCAATGCCTTCAAATCGAAGTCTATTGATGC }\end{array}$ \\
\hline M11 & $\begin{array}{l}\text { TATCTTCGATGGGTAAATGCTTGCCTGCGGTATGAGCTGAGGAACTATCA[G/A]C } \\
\text { CTGTCCCAGGCAAAACAACCGCAAGGGATCTCAGTAAAACGTTAAGCCC }\end{array}$ \\
\hline M12 & $\begin{array}{l}\text { GCGTACTCAATAGCCACCTTAGCTACATACTCACAGTCGC[A/G]GCGATATTGTTT } \\
\text { GCCTAGCATGTAGTGACCTACATAGTCT }\end{array}$ \\
\hline M13 & $\begin{array}{l}\text { CGTCATTGATTTTATATTTCATTTGTGCTTCCATTCTTGCTCATTTGCCA[T/C]AAG } \\
\text { CGCCGTACTATTATTGCAATTTCATGGTTCTGACCTTTGATCAGTC }\end{array}$ \\
\hline M13.4 & $\begin{array}{l}\text { AACTCAAATAAGCTTCAATTAGGATGGATTAGCTGGTCTAAGGATCCATTTCTTA } \\
\text { CTGTTCCCCATGTCTATGTCTACG[C/T]ACAGACTTCCACGATGACTAGAAGCCTA } \\
\text { TCATCTGTACGTGGAAGATTCCTTAATGTCTITAGATTAGATTAAGAAATGGCTCA } \\
\text { GTAACGAAGCTCAAGTGC }\end{array}$ \\
\hline M14 & $\begin{array}{l}\text { CAACGGCAATAAAGCTTTCATCAAGAAGATGACATGTITTGATTGGAGT[C/T]GT } \\
\text { TCTTCTTCATTAATTGATCGATTGTTTCTTIATCACCTCCACTTC }\end{array}$ \\
\hline M441 & $\begin{array}{l}\text { GGCAATTGGATTTACTGGGTTGGACCACTCGTTGGTGGTGGTCTGGCTGGTCTAG } \\
\text { TCTACAGTAA[C/T]GTGTACATGAACAACGATCATGTCCCTCTCTCTAGCGATITT } \\
\text { AAATTAATTGTTGAATTGATTGTGTTATGT }\end{array}$ \\
\hline M15 & $\begin{array}{l}\text { CAAGAATTGGATGAATTCTACAGAGACTTTGCGCAACAATTGGAATATCG[C/T]GA } \\
\text { ACAAAATGAGGTCCCGGAGAGAAACAACATCGATGAAGAATCATTAAA }\end{array}$ \\
\hline
\end{tabular}

${ }^{1} \mathrm{M} 8$ is a simple sequence repeat (SSR) marker (Hmps_E078) (Yi et al., 2006)

\section{Detached leaf assay}

Thrips resistance based on larval development was determined in a detached leaf assay, as previously described in chapter 2 . We selected the youngest fully opened leaves of each plant. We placed each detached leaf with the petiole in a droplet of $1.5 \%$ water agar in a Petri dish (BD Falcon, tight-fit lid $50 \times 9 \mathrm{~mm}$ ). Five 
synchronized L1s were placed on each leaf, after which the Petri dish was firmly closed and incubated in a growth cabinet at $25^{\circ} \mathrm{C}$. Thrips development was determined at 3, 5 and 7 days post infestation. The level of thrips resistance was determined by the fraction L1 that did not develop into second instar larvae (L2). Development of L1 into L2 occurs in 1-2 days under optimal conditions (Lublinkhof and Foster, 1977). Four leaves per F3 plant with a recombination of 12 weeks old plants were used to fine map the QTL as described by Maharijaya et al (2015). Three leaves per F3 plant (line 1-5, R or S) at a plant age of 12 weeks were used to validate this previously identified QTL region. After selfing the F3 plants that have a recombination within the newly mapped QTL region for one or two generations, three leaves per F4/F5 plant at a plant age of 10 weeks were used to further fine map and validate the new region.

To study the expression of genes in the QTL region upon induction with L1 larvae, the youngest fully opened leaves of 12 weeks old plants were collected and placed in Petri dishes as described above. Half of the leaves was infested with 15 synchronized L1s. After six hours, all leaves were frozen in liquid nitrogen and stored at $-80{ }^{\circ} \mathrm{C}$ until RNA extraction.

\section{RNA extraction and sequencing}

Plants from the F4-line 10 with the resistance (" $R$ ") or susceptibility ("S") allele in homozygous state were selected for RNA sequencing in two different treatments (i.e. induced: infested with 15 L1 larvae, or mock-treated: not infested). Fifteen plants with genotype $\mathrm{R}$ and fifteen plants with genotype $\mathrm{S}$ were randomized in the greenhouse. Leaves of the plants were collected and either infested or mocktreated, as described in the previous paragraph. Three pools of leaves from 5 plants with the same QTL genotype ("R" or " $S$ ") and treatment (induced or mock) were ground to a fine powder with mortar and pestle in liquid nitrogen. Total RNA was extracted from the pooled samples using RNAeasy Plus Mini Kit (QIAGEN) and sent to Novogene Technology Co., LTD (Hong Kong), for library preparation and sequencing. STAR (Dobin et al., 2012) was used to map the reads to the Zunla-1 assembly and the UCD10X assembly. Read count files were generated using HTSeq (Anders et al., 2014). The analysis of differentially expressed genes was done using SARTools in R-3.5.2 (R Core Team, 2018) (Varet et al., 2016). To analyse whether SNPs are predicted to lead to changes in protein structures, RNA sequences of each group (" $R$ " and " $S$ ") were translated into protein sequences using ExPASy (Gasteiger et al., 2003). SNPs that translated into different amino acids were analysed using PROVEAN tools to determine whether the change in amino acid is predicted to lead to a change in protein structure (Choi et al., 2012). 


\section{Data analysis and statistics}

Fractions $\mathrm{L} 1$ were transformed by $\mathrm{y}=\operatorname{arcsine}(\sqrt{ } \mathrm{x})$ before analysing the data. $A$ Student's t-test was used to determine whether the transformed fractions $L 1$ significantly differed between plants with the " $\mathrm{R}$ " or " $\mathrm{S}$ " allele within the same F3 line to validate the previously identified QTL region. Student's t-test was also used to determine significant differences in resistance between plants that had the recombination in homozygous state and plants with the " $\mathrm{R}$ " or " $\mathrm{S}$ " allele within each F4 or F5 line. All statistical analyses were conducted using Genstat $18^{\text {th }}$ edition (VSN International, 2015).

\section{Results}

\section{QTL validation}

To validate the previously identified QTL in the lines that were derived from the mapping population of Maharijaya et al. (2015), plants from five F3 lines that either have the resistance allele ("R") or susceptibility allele (" $S$ ") in homozygous state in the current QTL region were scored for first instar larval development. A high fraction L1 indicates thrips resistance. Plants with the " $R$ " allele showed a significantly higher fraction L1 compared to plants that have the " $\mathrm{S}$ " allele for all F3 lines (Figure 1).

\section{Fine mapping and validation}

In order to fine map the thrips resistance $Q T L$, fraction $L 1$ was determined for the 120 plants from F3 lines 1-10 with a recombination in heterozygous state in the 2-LOD interval of the QTL region, between markers M2 and M15 (Supplementary data Table S1). The highest correlation between larval development and marker score (RR, SS or RS) was found with marker M12.

In order to validate this result and to further narrow down the interval, $13 \mathrm{~F} 3$ plants with a recombination between M10 and M15 were selfed for one or two generation and the F4 and F5 plants with the recombination in homozygous state were selected. They were compared to plants from the same line with the " $\mathrm{R}^{\prime \prime}$ or "S" allele in homozygous state (Table 3). From the results of line 2009070-159 it appears that the causal gene is located to the right of marker M12, while from the results of line 247-309 we conclude that it is located to the left of marker M13.4. The physical distance is $0.39 \mathrm{Mbp}$ according to the Zunla-1 assembly and 0.40 Mbp according to the UCD10X assembly.

\section{Candidate genes}

The Genome Data Viewer of NCBI was used to list the candidate genes in the fine mapped QTL region (Table 4)(Bethesda (MD), 2012). Nine genes are predicted in 
the region between the flanking markers (M12 and M13.4) based on the $C$. annuum Zunla-1 assembly (Qin et al., 2014). In the UCD10X assembly of CM334, the region between M13.4 and M14 is inverted (Hulse-Kemp et al., 2018). Therefore, the six genes in the region between M13.4 and M14 are also included in the overview of candidate genes.

The RNAseq data of two groups of plants, i.e. with the resistance allele ("R") or the susceptibility allele ("S") in homozygous state, in two different treatments, i.e. induced or mock, were used to compare differentially expressed genes (DEGs) in the fine mapped QTL region. Two DEGs were found in the region of interest, i.e. LOC107876110, an organic cation transporter (OCT7) and LOC107874801, an uncharacterized locus (Figure 2A-B). We observed significantly lower counts of OCT7 reads in the " $S$ " group when leaves were induced with L1s compared to mock-treated leaves (Figure 2A). In the " $R$ " group, we did not observe significant differences between mock and induced leaves. We observed a significant increase in counts of the uncharacterized locus reads upon induction in the " $\mathrm{S}$ " group (Figure 2B) and no significant differences between the " $\mathrm{R}$ " groups with different treatments. Expression of other genes in the QTL region did not significantly differ between the groups or treatments (Supplementary data Table S2).

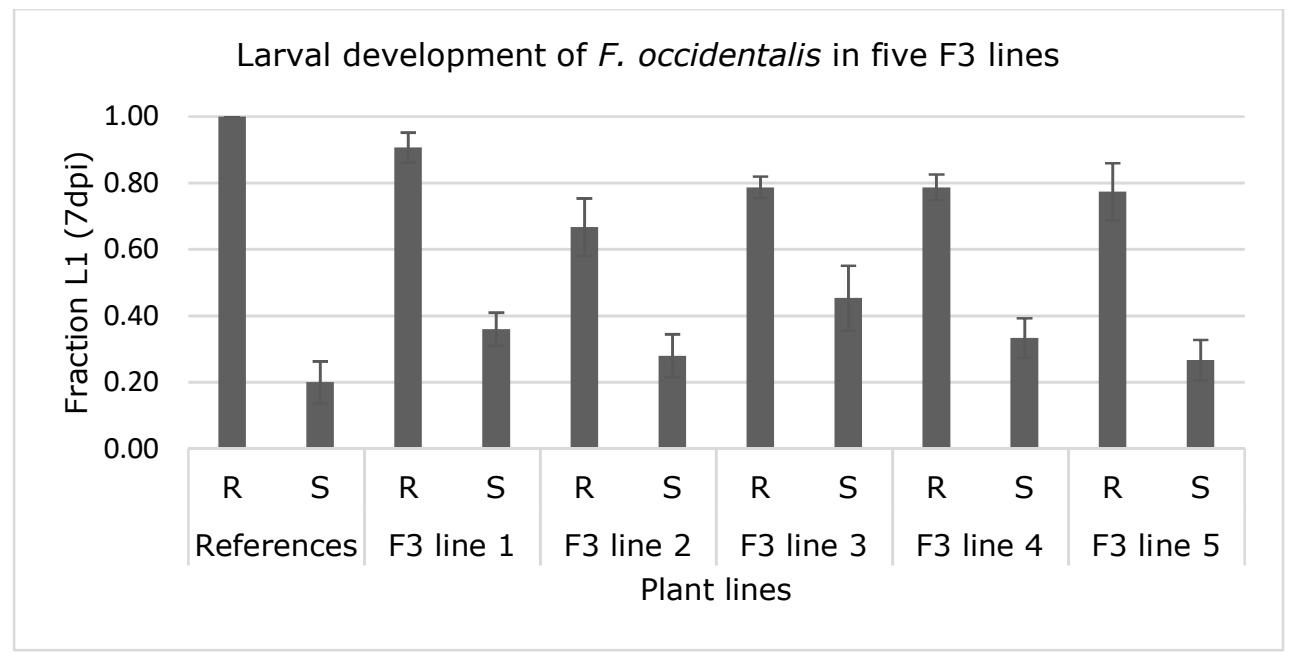

Figure 1: Validation of the QTL for thrips resistance in Capsicum. Larval development of $F$. occidentalis was studied on leaves of five $\mathrm{F} 3$ lines with either the resistance (" $\mathrm{R}$ ") or susceptibility ("S") allele in homozygous state. CGN16975A and CGN17219A were included as resistant ("R") and susceptible (" $S$ ") references. All differences between plants from group " $R$ " and group " $S$ " within the same line were significant $(P<0.05)$. 
in

믇

o.

高

कि

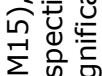

$\sum$ कू.

$+0$

을 究

$\sum \frac{\pi}{\omega} \frac{d}{0}$

这资

항워

है ते

당

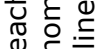

ᄒᄒ $\subseteq \stackrel{\square}{~}$

ᄂ

$0 \div$

$0 \stackrel{\bar{\sigma}}{0}$

ยิ

번 든

오는

인 궁

넝

0

ธ ข

늠

웅후

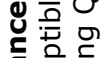

응.

出

佥

흥

ํㅡㄴ돈

농

$+60$

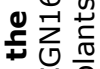

¿ U

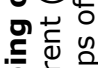

흔

응음

든

覀

돈

0

용

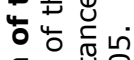

도 뽕웅

은 원원

흐

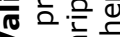

$>$ 至

$\ddot{m}$ 등

능 인

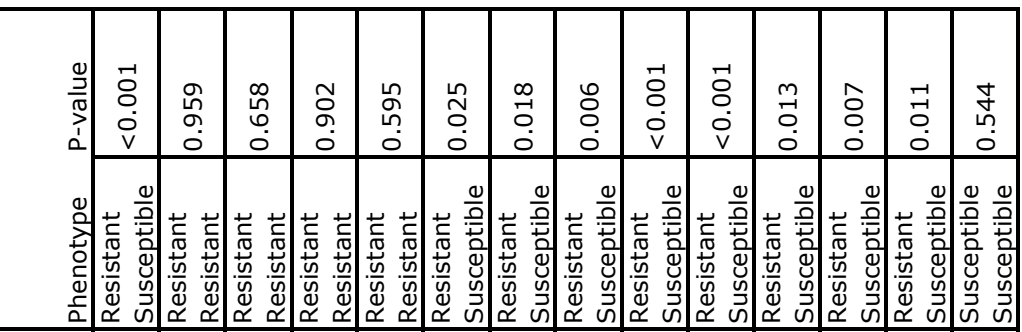

$\exists$

든

䒕 司同

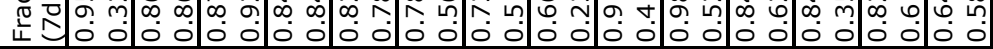

ㅇ.

$\sum$ 就 +

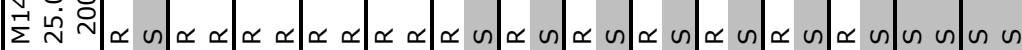
$\forall-7$.

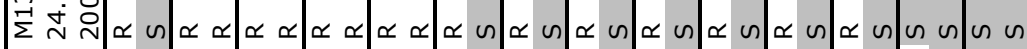
m

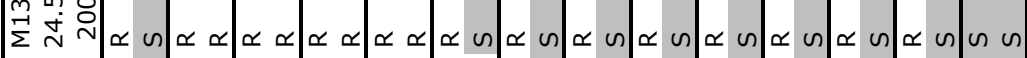

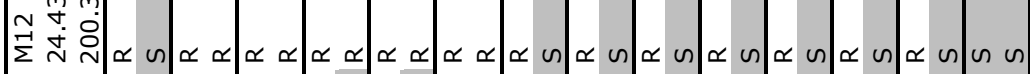

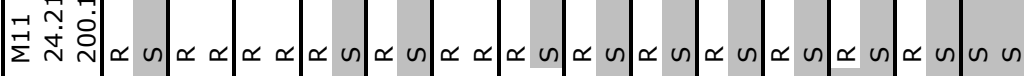

$\nabla$ o

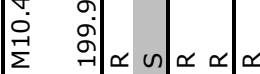

$\infty$

एं बे

$\sim \infty$

एक

$\rightarrow \quad \forall$

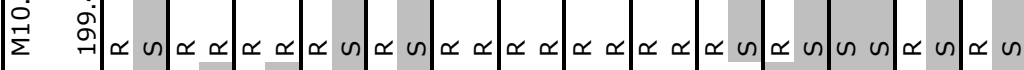
은

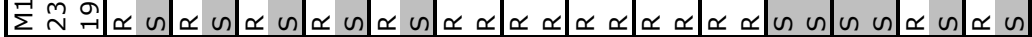
6 응 음

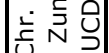

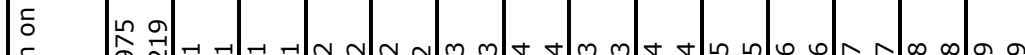

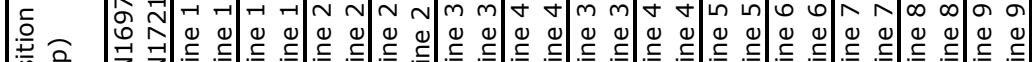


Three genes in our region of interest have SNPs that are predicted to lead to changes in protein structures, based on the analysis with ExPASy and PROVEAN (Table 5A-C), including the two genes found in the DEG analysis (OCT 7 and LOC107874801); the third gene is an acid phosphatase 1 (APS1).

Table 4: List of predicted genes in the fine mapped QTL region on chromosome 6. The gene list is based on the nine predicted genes on the Capsicum annuum Zunla-1 assembly for the region between marker M12 and M13.4. The six predicted genes between M13.4 and M14 are also included. The Genome Data Viewer from NCBI is used to enlist the predicted genes and their gene description. Expression data of these predicted genes can be found in Supplementary data (Table S2)

\begin{tabular}{|l|l|l|}
\hline $\begin{array}{l}\text { Location (Zunla-1, } \\
\text { chromosome 6) }\end{array}$ & Gene symbol & Gene description \\
\hline $24.42-24.43 \mathrm{Mbp}$ & LOC107876103 & acid phosphatase 1-like \\
\hline $24.46-24.47 \mathrm{Mbp}$ & LOC107876104 & uncharacterized LOC107876104 \\
\hline $24.52-24.52 \mathrm{Mbp}$ & LOC107876105 & profilin-2 \\
\hline $24.53-24.54 \mathrm{Mbp}$ & LOC107874799 & putative protein TPRXL \\
\hline $24.54-24.55 \mathrm{Mbp}$ & LOC107876106 & protein IQ-DOMAIN 31-like \\
\hline $24.56-24.56 \mathrm{Mbp}$ & LOC107876107 & hexokinase-2 \\
\hline $24.56-24.57 \mathrm{Mbp}$ & LOC107876108 & nuclear poly(A) polymerase 4-like \\
\hline $24.73-24.74 \mathrm{Mbp}$ & LOC107876109 & organic cation/carnitine transporter 7-like \\
\hline $24.81-24.82 \mathrm{Mbp}$ & LOC107876110 & organic cation/carnitine transporter 7-like \\
\hline $24.83-24.84 \mathrm{Mbp}$ & LOC107874800 & uncharacterized LOC107874800 \\
\hline $24.95-24.95 \mathrm{Mbp}$ & LOC107874801 & uncharacterized LOC107874801 \\
\hline $24.96-24.96 \mathrm{Mbp}$ & TRNAK-CUU & transfer RNA lysine (anticodon CUU) \\
\hline $24.97-24.97 \mathrm{Mbp}$ & LOC107873994 & F-box/LRR-repeat protein At3g59250-like \\
\hline $25.01-25.01 \mathrm{Mbp}$ & LOC107873995 & uncharacterized LOC107873995 \\
\hline $25.06-25.07 \mathrm{Mbp}$ & LOC107876111 & uncharacterized LOC107876111 \\
\hline
\end{tabular}

\section{Discussion}

\section{Validation and fine mapping of the resistance QTL}

The evaluation of F3 plants that have the resistance or susceptibility allele in homozygous state in the previously described QTL region on chromosome 6 (Maharijaya et al., 2015) showed that plants with the resistance allele had a significantly higher fraction $L 1$, indicating that larval development is impaired and thus confirming the previously defined QTL region. This (partial) inhibition of larval development affects population development, as only part of the L1 larvae is able to develop into the next larval stage on the leaves expressing the resistance. This way, the resistance mechanism not only plays a role in controlling the insect population growth through interruption of the thrips life cycle, but also in the spread of tospoviruses. Thrips can only acquire tospoviruses such as TSWV in the 
L1 stage or early L2 stage, and re-infect plants through their saliva during the adult stage (Whitfield et al., 2005), thus interrupting the development of L1 larvae into next developmental stage is a promising mechanism to prevent or limit tospovirus outbreaks. Previous thrips resistance QTL mapping studies used reproductive adaptation (Frei et al., 2005), damage score (Frei et al., 2005; OmoIkerodah, 2008; Muchero et al., 2010; Maharijaya et al., 2015; Sobda, 2017) or larval and pupal development and survival (Maharijaya et al., 2015) as parameters for thrips resistance. By validating the QTL region using larval development as a resistance parameter, we confirm that this method is appropriate to further fine map the resistance gene(s).

Fine mapping of the resistance QTL resulted in narrowing down our region of interest to an interval between markers M12 and M13.4. The physical distance between these markers is $0.39 \mathrm{Mbp}$, based on the Zunla-1 assembly and $0.40 \mathrm{Mbp}$ based on the UCD10X assembly (Qin et al., 2014; Hulse-Kemp et al, 2018). As we only found a few plants with a recombination between M12 and M13.4 in our screening of 2500 plants, thousands of plants would have to be screened to find plants with new recombinations between these two markers. Therefore, we used gene expression and gene functionality to further limit the number of candidate genes in the current interval.

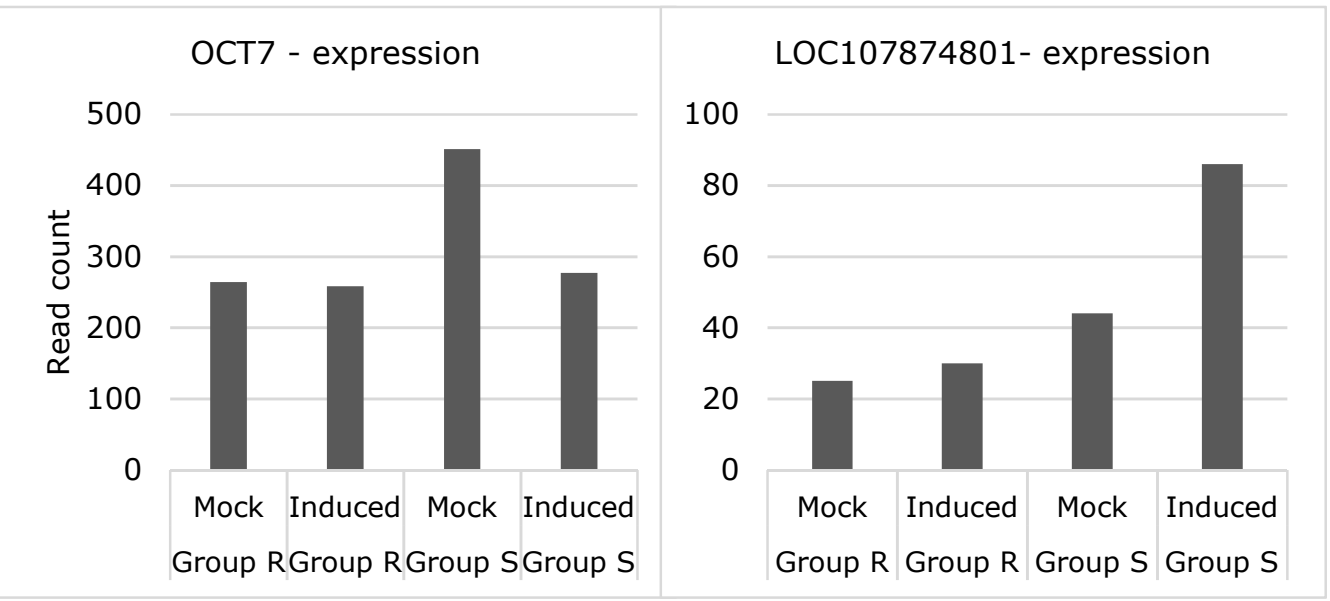

Figure 2: Differentially expressed genes in the fine mapped QTL region on chromosome 6. Group " $R$ " and " $S$ " indicate the groups of plants that have the resistance ("R") or susceptibility ("S") allele in homozygous state for the QTL region. "Induced" indicates the leaves that were induced with 15 L1 larvae for six hours, whereas no L1s were added to the "Mock" treated leaves. OCT7 and LOC107874801 show significant differences in gene counts between "Mock" and "Induced" treatments within "Group S" (P-adjusted for multiple comparisons <0.05). 


\begin{tabular}{|c|c|c|c|c|}
\hline $\begin{array}{l}\text { Amino acid } \\
\text { group } R\end{array}$ & $\begin{array}{l}\text { Amino acid } \\
\text { group S }\end{array}$ & Position & $\begin{array}{l}\text { PROVEAN } \\
\text { score }\end{array}$ & Prediction \\
\hline Glutamic acid & Lysine & 14 & -0.3 & Neutral \\
\hline Cysteine & Tyrosine & 216 & -7.321 & Deleterious \\
\hline Phenylalanine & Leucine & 482 & 0.578 & Neutral \\
\hline Valine & Deletion & $483-490$ & 2.237 & Neutral \\
\hline
\end{tabular}

\begin{tabular}{|l|l|l|l|l|}
\hline \multicolumn{4}{|l|}{ Table 5B - LOC107874801 } \\
\hline $\begin{array}{l}\text { Amino acid } \\
\text { group R }\end{array}$ & $\begin{array}{l}\text { Amino acid } \\
\text { group S }\end{array}$ & Position & $\begin{array}{l}\text { PROVEAN } \\
\text { score }\end{array}$ & Prediction \\
\hline Lysine & Glutamine & 3 & 0.550 & Neutral \\
\hline Aspartic acid & Asparagine & 169 & -4.722 & Deleterious \\
\hline
\end{tabular}

\begin{tabular}{|c|c|c|c|c|}
\hline $\begin{array}{l}\text { Amino acid } \\
\text { group } R\end{array}$ & $\begin{array}{l}\text { Amino acid } \\
\text { group S }\end{array}$ & Position & $\begin{array}{l}\text { PROVEAN } \\
\text { score }\end{array}$ & Prediction \\
\hline Valine & Isoleucine & 48 & -0.118 & Neutral \\
\hline Phenylalanine & Tyrosine & 131 & 2.828 & Neutral \\
\hline Glycine & Alanine & 137 & -5.119 & Deleterious \\
\hline Alanine & Proline & 159 & -3.747 & Deleterious \\
\hline
\end{tabular}

Table5A-C: SNPs in the fine mapped region of the QTL on chromosome 6 that are predicted to lead to changes in protein structure. The columns "Amino acid group $R$ " and

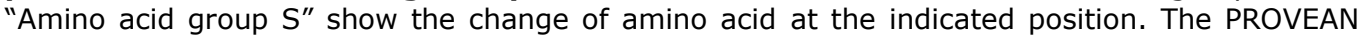
score indicates the prediction score that the changed amino acid leads to a change in protein structure. A PROVEAN score that is lower than the threshold indicates that the altered amino acid is likely to lead to a loss of function (deleterious). The threshold is set at -2.5 by default.

\section{Candidate genes}

Fifteen candidate genes are predicted in the current fine mapped QTL region on chromosome 6 . Five of the predicted genes are uncharacterized loci. Based on the differential gene expression analysis and the prediction of SNPs that lead to protein structural changes, three genes, of which one is an uncharacterized locus, are the most likely candidates in the resistance mechanism.

The first candidate is the predicted gene encoding a protein similar to acid phosphatase 1 (APS1). Acid phosphatase 1 is similar to vegetative storage protein 2 in Arabidopsis thaliana (AtVSP), an acid phosphatase which is induced upon wounding, insect feeding, methyl jasmonate and phosphate deprivation (Mason and Mullet, 1990; Berger et al., 1995; McConn et al., 1997; Stotz et al., 2000; Berger et al., 2002; Reymond et al., 2004). Due to its acid phosphatase activity, AtVSP causes developmental delays and increased mortality when included in the diet of insects with an acidic gut lumen (Liu et al., 2005). It is hypothesized that 
thrips have an acidic gut as well, as proteases present in the thrips midgut have an optimum at pH 3.5 (Annadana et al., 2002; Outchkourov et al., 2004). The proteins predicted to result from the susceptibility and resistance alleles of APS1 differ. When the predicted protein change in the susceptibility allele would result in a loss of function of the APS1 protein, it could explain the difference in thrips resistance that we observe when comparing plants with the susceptibility allele in homozygous state, compared to plants with the resistance allele in homozygous state.

The second candidate gene is an organic cation/carnitine transporter (OCT7), which plays a role in plant adaptation to environmental stresses such as cold stress, drought stress and salt stress in $A$. thaliana (Küfner and Koch, 2008). It can transport organic cations, nicotinate and compounds such as trigonelline (Berardini et al., 2015). Trigonelline has also been connected to thrips resistance. Mirnezhad et al. (2010) observed lower amounts of this compound in thripsresistant tomato accessions. They hypothesized that this was a trade-off to favour acyl sugar production. The proteins predicted to result from the susceptibility and resistance alleles of OCT7 differ. This might lead to non-functional or less than optimal functioning transporters, leading to disruptions of cation or carnitine balances and deficiencies. In Arabidopsis, a mutation in organic cation transporter 1 (AtOCT1) affected the expression of carnitine-related genes and led to developmental defects (Lelandais-Brière et al., 2007). Also, we observed that the level of expression of OCT7 in mock-treated plants that have the susceptibility allele in homozygous state was significantly higher than OCT7 expression in mocktreated plants with the resistance allele in homozygous state. The expression of OCT7 in the susceptible group of plants was decreased upon induction with L1 larvae. This might indicate that high expression of OCT7 in the susceptible group of plants favours larval development, but that plant carrying the resistance allele can induce a reduction in OCT7 expression upon recognition of L1 larvae.

The third candidate gene is an uncharacterized locus, LOC107874801. This candidate gene did not show significant difference in expression between mocktreated and L1 induced plants that have the resistance allele in homozygous state, but did show a significantly increased expression when plants with the susceptibility allele in homozygous state were induced with L1 larvae. This might indicate that LOC107874801 is a susceptibility gene that plays a role in increasing the compatibility between plant and insect, for instance by blocking the plant's defence pathway upon induction (Schie and Takken, 2014).

In addition, differences between the resistance and susceptibility allele in DNA sequences that are not transcribed into mRNA might affect gene expression. For instance, SNPs in enhancer regions might lead to differences in affinity for 
transcription factors, thus to differences in gene expression. Also, variants in the promotor region of the predicted genes might lead to differences in expression. Given that the enhancers and promotor regions are located within the boundaries of the fine mapped QTL, these variants should also be considered. As we only studied gene expression at one time point (6 hours), we might have missed genes that have differential expression between group $\mathrm{R}$ and group $\mathrm{S}$ with different treatments at earlier or later time points. For instance, Sarde (2019) showed that many changes in gene expression in pepper upon thrips feeding start $1 \mathrm{~h}$ and $2 \mathrm{~h}$ after infestation. Therefore, the potential role of other genes that are located in the QTL region between M12 and M13.4 should not be ignored.

\section{Conclusions}

In this study, we validated the previously identified QTL region on chromosome 6 using larval development as a resistance parameter. The QTL region was fine mapped to a region of $0.4 \mathrm{Mbp}$. Fifteen candidate genes were found in this region. Combining a QTL mapping study with other methods such as RNA-sequencing can result in a powerful tool for exploring QTL regions and for selecting candidate genes. In this study, we used the RNA-sequencing approach to find DEGs and SNPs that are predicted to lead to changes in protein structure. This resulted in the selection of three candidate genes that are likely to play a role in thrips resistance. OCT7 and LOC107874801 showed altered gene expression upon induction with first instar larvae in susceptible plants, whereas no significant differences in gene expression were observed in resistant plants. Furthermore, OCT7, LOC107874801 and APS1 have SNPs that are predicted to lead to protein changes, which might indicate loss of function. Further validation of the altered gene expression and predicted protein structures is needed to confirm our findings. The selection of candidate genes is a first step in further elucidating the mechanism of thrips resistance in Capsicum.

\section{Acknowledgment}

We thank Betty Henken, Wendy van 't Westende and Mengjing Sun for their assistance with carrying out the detached leaf assays with the fine mapping (BH) and with the induction of leaf material used for RNA-seq (BH, WvtW and MS). This project was financially supported by the Dutch Topsector Horticulture \& Starting Materials TKI-grant (TKI-1409-045), from the Ministery of Economic Affairs of the Netherlands, and by breeding companies Nunhems Netherlands B.V. and Bejo Zaden B.V.. Within the Topsector, private industry, knowledge institutes and the government are working together on innovations for sustainable production of safe and healthy food and the development of a healthy green environment. 


\section{Author contributions}

$\mathrm{PVH}, \mathrm{BV}, \mathrm{REV}$ and JvL conceived and designed the experiments. MvK processed the RNAseq data and contributed to designing the molecular markers. PvH carried out the experimental work, data analysis and wrote the first draft of the manuscript. BV, REV and JvL were involved in revision of the manuscript.

\section{Supplementary data}

\section{Table S1: Overview of the genotypes and phenotypes of plants which were selected}

to fine map the QTL on chromosome 6. Each individual line represents a single plant with a recombination between the two flanking markers of the previously identified QTL region (Maharijaya et al., 2015). "RR" indicates plants that show the same allele as the resistant accession CGN16975 in homozygous state for the corresponding marker. "SS" indicates plants with the same allele as the susceptible accession CGN17219 in homozygous state for the corresponding marker. When plants score heterozygous for a marker, they are scored with "RS". M11 could not distinguish marker score "RS" or "SS", thus these scores are indicated as "RS/SS". Unknown marker scores are marked as "U". Unknown "Fraction L1 (7dpi) are marked as "*". High fractions L1 (7dpi) indicate inhibition in larval development from the L1 into the L2 stage.

\begin{tabular}{|c|c|c|c|c|c|c|c|c|c|c|c|}
\hline Zunla & M2 & M6 & M7 & M8 & M10 & M11 & M12 & M13 & M14 & M15 & \begin{tabular}{|l} 
Fraction \\
L1 \\
(7dpi)
\end{tabular} \\
\hline $\begin{array}{l}\text { Position } \\
\text { (Mbp) }\end{array}$ & $\begin{array}{l}\text { Chr0 } \\
6 \\
\end{array}$ & Chr06 & Chr06 & Chr06 & Chr06 & Chr06 & Chr06 & Chr06 & Chr06 & Chr06 & \\
\hline Plant line & 22.3 & 22.7 & 22.7 & 22.8 & 23.6 & 24.2 & 24.4 & 24.6 & 25.1 & 28.2 & \\
\hline CGN16975 & RR & RR & RR & RR & RR & RR & RR & $\overline{R R}$ & RR & RR & 1.00 \\
\hline CGN17219 & SS & SS & SS & SS & SS & SS & SS & SS & SS & SS & 0.00 \\
\hline 2009119 & RR & RR & RR & $\mathrm{RR}$ & $\mathrm{U}$ & RR & RR & RR & RR & RS & 0.64 \\
\hline 2009073 & RR & RR & RR & $R R$ & RR & RR & RR & RR & RR & RS & 1.00 \\
\hline 2009171 & RR & RR & RR & $\mathrm{RR}$ & RR & RR & RR & RR & RR & RS & 0.80 \\
\hline 2009209 & RR & RR & RR & RR & RR & RR & RR & RR & RR & RS & 0.70 \\
\hline 2009070 & RR & RR & RR & $R R$ & RR & RR & RR & RR & RR & RS & 0.60 \\
\hline 2009070 & RR & RR & RR & $\mathrm{RR}$ & RR & RR & RR & RS & RS & RS & 0.80 \\
\hline 2009111 & RR & RR & RR & $R R$ & RR & RS/SS & RS & RS & RS & RS & $*$ \\
\hline 2009247 & RR & RR & RR & RR & RR & RS & RS & RS & RS & RS & $*$ \\
\hline 2009247 & RR & RR & RR & $R R$ & RR & RS & RS & RS & RS & RS & * \\
\hline 2009247 & RR & RR & RR & $\mathrm{RR}$ & RR & $\mathrm{U}$ & RS & RS & RS & RS & $*$ \\
\hline 2009247 & RR & RR & RR & $R R$ & RR & RS & RS & RS & RS & RS & $*$ \\
\hline 2009247 & RR & RR & RR & $\mathrm{RR}$ & RR & RS & RS & RS & RS & RS & $*$ \\
\hline 2009233 & RR & RR & RR & RR & RR & RS/SS & RS & RS & RS & RS & 1.00 \\
\hline 2009247 & RR & RR & RR & RR & RR & RS & RS & RS & RS & RS & 1.00 \\
\hline 2009111 & RR & RR & RR & $\mathrm{RR}$ & RR & RS/SS & RS & RS & RS & RS & 0.80 \\
\hline 2009119 & RR & RR & RR & $\mathrm{RR}$ & RR & RS/SS & RS & RS & RS & RS & 0.80 \\
\hline 2009111 & RR & RR & RR & RR & RR & RS/SS & RS & RS & RS & RS & 0.22 \\
\hline 2009111 & RR & RR & RR & RR & RR & RS/SS & RS & RS & RS & RS & 0.20 \\
\hline 2009070 & RR & RR & RR & RR & RR & RS/SS & RS & RS & RS & RS & 0.00 \\
\hline 2009247 & RR & RR & RR & RR & RR & RS & RS & RS & RS & RS & 0.00 \\
\hline 2009209 & RR & RR & RR & $R R$ & RR & RS/SS & RS & RS & RS & RS & 0.70 \\
\hline 2009247 & RR & RR & RR & RR & RR & $\mathrm{U}$ & RS & RS & RS & RS & 0.00 \\
\hline 2009247 & RR & RR & RR & RR & RR & RS & RS & RS & RS & RS & 0.00 \\
\hline
\end{tabular}




\begin{tabular}{|c|c|c|c|c|c|c|c|c|c|c|c|}
\hline Zunla & M2 & M6 & M7 & M8 & M10 & M11 & M12 & M13 & M14 & M15 & \begin{tabular}{|l|} 
Fraction \\
L1 \\
(7dpi)
\end{tabular} \\
\hline $\begin{array}{l}\text { Position } \\
\text { (Mbp) }\end{array}$ & $\begin{array}{l}\text { Chr0 } \\
6\end{array}$ & Chr06 & Chr06 & Chr06 & Chr06 & Chr06 & Chr06 & Chr06 & Chr06 & Chr06 & \\
\hline Plant line & 22.3 & 22.7 & 22.7 & 22.8 & 23.6 & 24.2 & 24.4 & 24.6 & 25.1 & 28.2 & \\
\hline 2009171 & RR & RR & RR & RR & RS & RS/SS & RS & RS & RS & RS & 1.00 \\
\hline 2009233 & RR & RR & RR & RR & RS & RS/SS & RS & RS & RS & RS & 0.00 \\
\hline 2009111 & RR & RR & RR & RR & RS & RS/SS & RS & RS & RS & RS & 0.40 \\
\hline 2009119 & RR & RR & RR & RR & RS & RS/SS & RS & RS & RS & RS & 0.80 \\
\hline 2009111 & RR & RR & RR & RS & RS & RS/SS & RS & RS & RS & RS & 0.40 \\
\hline 2009111 & RR & RR & RS & RS & RS & $\mathrm{RS} / \mathrm{SS}$ & RS & RS & RS & RS & 0.40 \\
\hline 2009247 & RR & $\mathrm{U}$ & U & $\mathrm{U}$ & RS & $U$ & RS & RS & RS & RS & 0.00 \\
\hline 2009247 & RR & $\mathrm{U}$ & $\mathrm{U}$ & $\mathrm{U}$ & RS & RS & RS & RS & RS & RS & 1.00 \\
\hline 2009209 & RR & RS & RS & RS & RS & RS/SS & RS & RS & RS & RS & 0.70 \\
\hline 2009171 & RR & RS & RS & RS & RS & RS/SS & RS & RS & RS & RS & 0.60 \\
\hline 2009070 & RR & RS & RS & RS & RS & RS/SS & RS & RS & RS & RS & 0.60 \\
\hline 2009208 & RR & RS & RS & RS & RS & RS/SS & RS & RS & RS & RS & 0.00 \\
\hline 2009247 & RS & RS & RS & RS & $\mathrm{U}$ & $U$ & $\mathrm{U}$ & U & $\mathrm{U}$ & RS & 0.00 \\
\hline 2009111 & RS & RR & RR & RR & RR & RR & RR & RR & RR & RR & 0.50 \\
\hline 2009070 & RS & RR & RR & RR & RR & RR & RR & RR & RR & RR & 1.00 \\
\hline 2009119 & RS & RS & RS & RR & RR & RR & RR & RR & RR & RR & 0.40 \\
\hline 2009070 & RS & RS & RS & RS & RR & RR & RR & RR & RR & RR & 1.00 \\
\hline 2009233 & RS & RS & RS & RS & RR & RR & RR & RR & RR & RR & 1.00 \\
\hline 2009171 & RS & $\mathrm{U}$ & $\mathrm{U}$ & $\mathrm{U}$ & RR & RR & RR & RR & RR & RR & 1.00 \\
\hline 2009233 & RS & $\mathrm{U}$ & U & $\mathrm{U}$ & RR & RR & RR & RR & RR & RR & 1.00 \\
\hline 2009111 & RS & $\mathrm{U}$ & $\mathrm{U}$ & $\mathrm{U}$ & RR & RR & RR & RR & RR & RR & 1.00 \\
\hline 2009171 & RS & RS & RS & RS & RR & RR & RR & RR & RR & RR & 0.80 \\
\hline 2009247 & RS & RS & RS & RS & RS & RR & RR & RR & RR & RR & $*$ \\
\hline 2009094 & RS & RS & RS & RS & RS & RR & RR & RR & RR & RR & 1.00 \\
\hline 2009094 & RS & RS & RS & RS & RS & RS/SS & RR & RR & RR & RR & 1.00 \\
\hline 2009209 & RS & RS & RS & RS & RS & RR & RR & RR & RR & RR & 1.00 \\
\hline 2009119 & RS & RS & RS & RS & RS & RR & RR & RR & RR & RR & 0.80 \\
\hline 2009119 & RS & RS & RS & RS & RS & RR & RR & RR & RR & RR & 0.80 \\
\hline 2009119 & RS & RS & RS & RS & RS & RR & RR & RR & RR & RR & 0.80 \\
\hline 2009119 & RS & RS & RS & RS & RS & RR & RR & RR & RR & RR & 0.60 \\
\hline 2009247 & RS & RS & RS & RS & RS & RS & RR & RR & RR & RR & 1.00 \\
\hline 2009111 & RS & RS & RS & RS & RS & RS/SS & RS & RS & RR & RR & 0.40 \\
\hline 2009088 & RS & RS & RS & RS & RS & RS/SS & RS & RS & RS & RR & 0.90 \\
\hline 2009088 & RS & RS & RS & RS & RS & RS/SS & RS & RS & RS & RR & 0.90 \\
\hline 2009119 & RS & RS & RS & RS & RS & RS/SS & RS & RS & RS & RR & 0.80 \\
\hline 2009209 & RS & RS & RS & RS & RS & RS/SS & RS & RS & RS & RR & 0.75 \\
\hline 2009088 & RS & RS & RS & RS & RS & RS/SS & RS & RS & RS & RR & 0.60 \\
\hline 2009088 & RS & RS & RS & RS & RS & RS/SS & RS & RS & RS & RR & 0.60 \\
\hline 2009088 & RS & RS & RS & RS & RS & RS/SS & SS & SS & SS & RR & 0.50 \\
\hline 2009247 & RS & RS & RS & RS & RS & $\mathrm{U}$ & SS & RS & RS & RS & 0.00 \\
\hline 2009111 & RS & RS & RS & RS & RS & RS/SS & RS & RS & RS & SS & 1.00 \\
\hline 2009233 & RS & RS & RS & RS & RS & RS/SS & RS & RS & RS & SS & 0.70 \\
\hline 2009119 & RS & RS & RS & RS & RS & RS/SS & RS & RS & RS & SS & 0.60 \\
\hline 2009094 & RS & RS & RS & RS & RS & RS/SS & RS & RS & RS & SS & 0.40 \\
\hline 2009070 & RS & RS & RS & RS & RS & RS/SS & RS & RS & RS & SS & 0.40 \\
\hline 2009209 & RS & RS & RS & RS & RS & RS/SS & RS & RS & RS & SS & 0.20 \\
\hline 2009094 & RS & RS & RS & RS & RS & RS/SS & RS & SS & SS & SS & 0.50 \\
\hline 2009119 & RS & RS & RS & RS & RS & RS/SS & SS & SS & SS & SS & 0.80 \\
\hline 2009073 & RS & RS & RS & RS & RS & RS/SS & RS & RS & SS & SS & 0.33 \\
\hline
\end{tabular}




\begin{tabular}{|c|c|c|c|c|c|c|c|c|c|c|c|}
\hline Zunla & M2 & M6 & M7 & M8 & M10 & M11 & M12 & M13 & M14 & M15 & \begin{tabular}{|l} 
Fraction \\
L1 \\
(7dpi)
\end{tabular} \\
\hline $\begin{array}{l}\text { Position } \\
\text { (Mbp) }\end{array}$ & $\begin{array}{l}\text { Chr0 } \\
6\end{array}$ & Chr06 & Chr06 & Chr06 & Chr06 & Chr06 & Chr06 & Chr06 & Chr06 & Chr06 & \\
\hline Plant line & 22.3 & 22.7 & 22.7 & 22.8 & 23.6 & 24.2 & 24.4 & 24.6 & 25.1 & 28.2 & \\
\hline 2009070 & RS & RS & RS & RS & RS & RS/SS & SS & $\overline{S S}$ & SS & $\overline{S S}$ & 0.60 \\
\hline 2009209 & RS & RS & RS & RS & RS & RS/SS & SS & SS & SS & SS & 0.60 \\
\hline 2009070 & RS & RS & RS & RS & RS & RS/SS & SS & SS & SS & SS & 0.60 \\
\hline 2009094 & RS & RS & RS & RS & RS & RS/SS & SS & SS & SS & SS & 0.50 \\
\hline 2009209 & RS & RS & RS & RS & RS & RS/SS & SS & SS & SS & SS & 0.40 \\
\hline 2009073 & RS & RS & RS & RS & RS & RS/SS & SS & SS & SS & SS & 0.30 \\
\hline 2009247 & RS & RS & RS & RS & RS & SS & SS & SS & SS & SS & 0.00 \\
\hline 2009247 & RS & RS & RS & RS & RS & SS & SS & SS & SS & SS & 0.00 \\
\hline 2009247 & RS & RS & RS & RS & RS & SS & SS & SS & SS & SS & 0.00 \\
\hline 2009247 & RS & RS & RS & RS & RS & SS & SS & SS & SS & SS & 0.00 \\
\hline 2009209 & RS & RS & RS & RS & SS & RS/SS & SS & SS & SS & SS & 0.00 \\
\hline 2009247 & RS & $U$ & $\mathrm{U}$ & $U$ & SS & SS & SS & SS & SS & SS & 0.00 \\
\hline 2009247 & RS & $U$ & $\mathrm{U}$ & $U$ & SS & SS & SS & SS & SS & SS & 0.00 \\
\hline 2009209 & RS & RS & RS & SS & SS & $\mathrm{RS} / \mathrm{SS}$ & SS & SS & SS & SS & 0.60 \\
\hline 2009233 & RS & RS & RS & SS & SS & RS/SS & SS & SS & SS & SS & 0.00 \\
\hline 2009094 & RS & RS & RS & SS & SS & RS/SS & SS & SS & SS & SS & 0.40 \\
\hline 2009111 & RS & RS & RS & SS & SS & RS/SS & SS & SS & SS & SS & 0.00 \\
\hline 2009070 & RS & SS & SS & SS & SS & RS/SS & SS & SS & SS & SS & 0.60 \\
\hline 2009119 & RS & SS & SS & SS & SS & RS/SS & SS & SS & SS & SS & 0.30 \\
\hline 2009247 & RS & $\mathrm{U}$ & $\mathrm{U}$ & $\mathrm{U}$ & $U$ & SS & SS & SS & SS & SS & 0.00 \\
\hline 2009247 & SS & $U$ & $\mathrm{U}$ & $U$ & RS & RS & RS & RS & RS & RS & 1.00 \\
\hline 2009233 & SS & RS & RS & RS & RS & RS/SS & RS & RS & RS & RS & 0.80 \\
\hline 2009094 & SS & SS & SS & RS & RS & RS/SS & RS & RS & RS & RS & 0.40 \\
\hline 2009209 & SS & SS & SS & RS & RS & $\mathrm{RS} / \mathrm{SS}$ & RS & RS & RS & RS & 0.40 \\
\hline 2009119 & SS & SS & SS & RS & RS & RS/SS & RS & RS & RS & RS & 0.40 \\
\hline 2009247 & SS & $\mathrm{U}$ & $\mathrm{U}$ & U & RS & RS & RS & RS & RS & RS & 0.00 \\
\hline 2009094 & SS & SS & SS & SS & RS & RS/SS & RS & RS & RS & RS & 1.00 \\
\hline 2009247 & SS & SS & SS & SS & SS & RS & RS & RS & RS & RS & * \\
\hline 2009247 & SS & SS & SS & SS & SS & $\mathrm{U}$ & RS & RS & RS & RS & * \\
\hline 2009094 & SS & SS & SS & SS & SS & $\mathrm{RS} / \mathrm{SS}$ & RS & RS & RS & RS & 0.60 \\
\hline 2009209 & SS & SS & SS & SS & SS & RS/SS & RS & RS & RS & RS & 0.60 \\
\hline 2009209 & SS & SS & SS & SS & SS & RS/SS & RS & RS & RS & RS & 0.50 \\
\hline 2009171 & SS & SS & SS & SS & SS & $\mathrm{RS} / \mathrm{SS}$ & RS & RS & RS & RS & 0.40 \\
\hline 2009209 & SS & SS & SS & SS & SS & RS/SS & RS & RS & RS & RS & 0.40 \\
\hline 2009070 & SS & SS & SS & SS & SS & RS/SS & RS & RS & RS & RS & 0.40 \\
\hline 2009209 & SS & SS & SS & SS & SS & RS/SS & RS & RS & RS & RS & 0.20 \\
\hline 2009111 & SS & SS & SS & SS & SS & $\mathrm{RS} / \mathrm{SS}$ & RS & RS & RS & RS & 0.20 \\
\hline 2009247 & SS & SS & SS & SS & SS & RS & RS & RS & RS & RS & 0.00 \\
\hline 2009247 & SS & SS & SS & SS & SS & $U$ & RS & RS & RS & RS & 0.00 \\
\hline 2009247 & $\overline{S S}$ & SS & SS & SS & SS & RS & RS & RS & RS & RS & 0.00 \\
\hline 2009070 & SS & SS & SS & SS & SS & RS/SS & SS & RS & RS & RS & 0.67 \\
\hline 2009119 & SS & SS & SS & SS & SS & $\mathrm{RS} / \mathrm{SS}$ & SS & SS & SS & RS & 0.60 \\
\hline 2009070 & SS & SS & SS & SS & SS & RS/SS & SS & SS & SS & RS & 0.60 \\
\hline 2009209 & SS & SS & SS & SS & SS & RS/SS & SS & SS & SS & RS & 0.60 \\
\hline 2009171 & SS & SS & SS & SS & SS & RS/SS & SS & SS & SS & RS & 0.60 \\
\hline 2009111 & SS & SS & SS & SS & SS & RS/SS & SS & SS & SS & RS & 0.00 \\
\hline
\end{tabular}


Table S2: Read counts for the predicted genes in the QTL region between M12 and M14. Reads were counted for plants from Group R (with the resistance allele in homozygous state) and Group S (with the susceptibility allele in homozygous state) for both treatments (i.e. mock-treated and induced with L1s). Significant differences in gene expression were only observed for LOC107876110 (OCT7) and LOC107874801 (uncharacterized locus). Predicted genes from which no read counts were obtained are indicated with "-".

\begin{tabular}{|l|l|l|l|l|l|}
\hline Gene symbol & Gene description & $\begin{array}{l}\text { Group } \\
\text { R } \\
\text { mock }\end{array}$ & $\begin{array}{l}\text { Group } \\
\text { mock } \\
\text { mock }\end{array}$ & $\begin{array}{l}\text { Group } \\
\text { induced } \\
\text { ind }\end{array}$ & $\begin{array}{l}\text { Group } \\
\text { induced }\end{array}$ \\
\hline LOC107876103 & acid phosphatase 1-like & 292 & 154 & 280 & 186 \\
\hline LOC107876104 & uncharacterized LOC107876104 & - & - & - & - \\
\hline LOC107876105 & profilin-2 & 20 & 27 & 12 & 15 \\
\hline LOC107874799 & putative protein TPRXL & 284 & 201 & 229 & 187 \\
\hline LOC107876106 & protein IQ-DOMAIN 31-like & 32 & 46 & 22 & 34 \\
\hline LOC107876107 & hexokinase-2 & 2236 & 1990 & 2290 & 2334 \\
\hline LOC107876108 & nuclear poly(A) polymerase 4-like & - & - & - & - \\
\hline LOC107876109 & organic cation/carnitine transporter 7-like & 2 & 11 & 2 & 3 \\
\hline LOC107876110 & organic cation/carnitine transporter 7-like & 264 & 451 & 241 & 291 \\
\hline LOC107874800 & uncharacterized LOC107874800 & - & - & - & - \\
\hline LOC107874801 & uncharacterized LOC107874801 & 25 & 44 & 30 & 86 \\
\hline TRNAK-CUU & transfer RNA lysine (anticodon CUU) & - & - & - & - \\
\hline LOC107873994 & F-box/LRR-repeat protein At3g59250-like & - & - & - & - \\
\hline LOC107873995 & uncharacterized LOC107873995 & - & - & - & - \\
\hline LOC107876111 & uncharacterized LOC107876111 & 684 & 1207 & 819 & 1096 \\
\hline
\end{tabular}





\section{CHAPTER 4}

\section{sin 40}

it

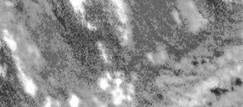

$=-2,1^{2}+t^{2}$

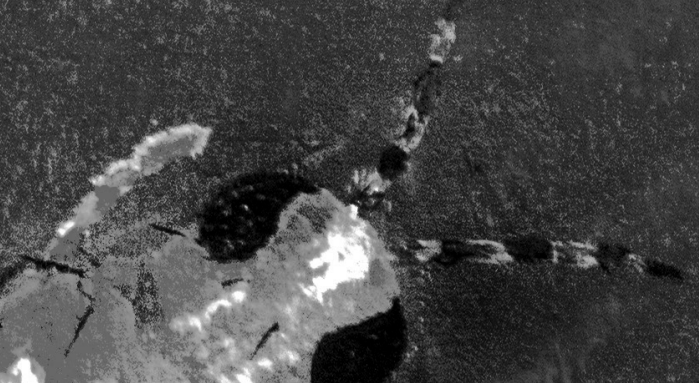




\section{The role of diterpene glycosides in the resistance mechanism controlled by a thrips resistance QTL in Capsicum}

Pauline van Haperen, Roeland E. Voorrips, Henriëtte D.L.M. van Eekelen, Ric C.H. de Vos, Joop J.A. van Loon, Ben Vosman 


\begin{abstract}
Thrips are important pest insects that can cause damage in pepper cultivation worldwide. Two independent studies in Capsicum identified diterpene glycosides as metabolites that are correlated with thrips resistance. The objectives of this study were to determine whether diterpene glycosides, or other metabolites, play a role in the thrips resistance mechanism encoded by genes located in a thrips resistance QTL on chromosome 6 . We did not observe significant differences in diterpene glycoside abundance between plants with the resistance allele and plants with the susceptibility allele in homozygous state for the QTL region, and between leaves exposed to first instar larvae and mock-treated samples. We did observe differences in diterpene glycoside abundance between the resistant and susceptible references. Our findings suggest that the resistance mechanism controlled by the thrips resistance QTL on chromosome 6 works independently from the diterpene glycoside pathway.
\end{abstract}




\section{Introduction}

Several thrips species are important pest insects that can cause severe damage to fruits, leaves and flowers in multiple crops worldwide (Kirk and Terry, 2003). They are minute insects that damage the plant directly through feeding, leading to silvering scars, malformation, altered carbon allocation and thus decreased yield (Welter et al., 1990; Shipp et al., 1998a). Thrips are difficult to control, because of their high reproduction rate, short life cycle, thigmotactic behaviour, their ability to feed on multiple host plants and to develop resistance to insecticides (Hansen et al., 2003; Bielza, 2008). Therefore, they are a threat to a large number of crops, including vegetables, fruits and ornamentals, and host plant resistance to thrips is a desired trait for breeders.

Several studies have identified sources of host plant resistance to thrips in different crops. Resistance quantitative trait loci (QTLs) have been mapped in common bean (Frei et al., 2005), cowpea (Omo-Ikerodah, 2008; Muchero et al., 2010; Sobda, 2017) and pepper (Maharijaya et al., 2015).

Several studies focussed on the potential role of secondary plant metabolites in thrips resistance. Leiss et al. (2009a) showed that concentrations of the pyrrolizidine alkaloids (PA) jacobine and jaconine and the flavonoid kaempferol glucoside were higher in resistant Senecio plants. In thrips-resistant chrysanthemum, higher concentrations of chlorogenic acid (caffeoyl quinic acid) and feruloyl quinic acid were found (Leiss et al., 2009b). Moreover, an unsaturated isobutylamide was suggested to repel thrips in chrysanthemum (Tsao et al., 2005). In the leaves of thrips-resistant carrots, high amounts of luteolin, sinapic acid and B-alanine were found (Leiss et al., 2013). Mirnezhad et al. (2010) and Vosman et al. (2018) showed that acylsugars are associated with thrips resistance in tomato.

In pepper leaves, Maharijaya et al. (2019) combined QTL mapping and an untargeted metabolomics approach to detect metabolite QTLs (mQTLs). Six mQTLs, of which four metabolites were correlated with thrips resistance, colocated with the thrips resistance QTL that was previously mapped to chromosome 6 (Maharijaya et al., 2015). These four metabolites included two acyclic diterpene glycosides and a flavonoid conjugate. In an independent study by Macel et al. (2019), monomer and dimer acyclic diterpene glycosides were identified as metabolites related to thrips resistance when comparing leaves of thrips-resistant and susceptible Capsicum accessions. Altogether, these two studies suggest a possible role of diterpene glycosides (capsianosides) in thrips resistance in Capsicum. 
The initial steps in the diterpene glycoside biosynthesis occur through the methylerythritol 4-phosphate (MEP) pathway in the plastids (Lange et al., 2000). Along the MEP pathway, the precursors of the terpenoids, i.e. isopentenyl diphosphate (IPP) and dimethylallyl diphosphate (DMAPP) are produced (Supplementary data Table S1A). Condensation of one DMAPP molecule and three IPP molecules yields geranylgeranyl pyrophosphate (GGPP), which is needed to synthesize diterpenes (Bohlmann et al., 1998; Takahashi and Koyama, 2006). These precursors are the substrates of enzymes called terpene synthases (TPSs) for the formation of terpenes (Chen et al., 2011)(Supplementary data Table 1B). Other enzymes such as Cytochrome P450 monooxygenases and UDP-glycosyl transferases (UGTs) also play a role in terpenoid glycoside synthesis (Collu et al., 2001; Richman et al., 2005).

The goal of this study is to determine if diterpene glycosides can be linked to the fine-mapped thrips resistance QTL in Capsicum. In chapter 3, the fine mapping of the resistance QTL identified by Maharijaya et al. (2015) resulted in a list of 15 candidate genes. Among these 15 genes, expression data and predicted loss of protein function due to sequence polymorphisms indicated three likely candidate genes; however the resistance mechanisms controlled by genes located in this QTL remain unknown. An untargeted metabolomics approach was used to determine whether or not diterpene glycosides, or other metabolites, are more abundant in F4 plants derived from the mapping population of Maharijaya et al. (2015) that contain the resistance allele for the QTL region in homozygous state, compared to plants from the same F4 line that have the susceptibility allele in homozygous state. In addition, we determined whether genes known to play a role in diterpene glycoside biosynthesis are located in the QTL region as defined by Maharijaya et al. (2015) and in the fine-mapped QTL region as defined in Chapter 3.

\section{Material and methods}

\section{Plant material}

We used the same plant material for metabolomics analysis as was previously used for RNA sequencing (F4 line 10, chapter 3). An F3 plant that was heterozygous for the entire fine-mapped QTL region on chromosome 6 (chapter 3) was selfed, and the next generation F4 plants that either had the resistance or susceptibility allele in homozygous state were selected in order to study differences in metabolite composition and/or content between these groups of plants within the same F4 line. The first generation selfing of a plant from the resistant accession CGN16975 (CGN16975A) and the second generation selfing from the susceptible accession CGN17219 (CGN17219A) were included as resistant and susceptible reference. Seeds were sown in potting compost in a 
greenhouse of Unifarm, Wageningen University and Research, Wageningen, The Netherlands. The plants were grown at a photoperiod of L16:D8 and 70\% RH at $25^{\circ} \mathrm{C}$. Thrips were controlled using Orius laevigatus (Fieber) (Entocare C.V., Wageningen, The Netherlands). Plants were watered three times per week. Two times per week, nutrients were added.

\section{Molecular markers and plant selection}

Young leaves from the plants were used to extract DNA and to determine the plant's genotype for the fine-mapped QTL region (Chapter 3). Two SNP markers, i.e. M11 and Isotig 18067-441, were used to determine which plants have the resistance allele (" $R$ ") or the susceptibility allele (" $S$ ") in homozygous state for the entire QTL region on chromosome 6 (Table 1 ). We selected 15 plants that had the "R" allele, and 15 plants that had the " $\mathrm{S}$ " allele.

Table 1: SNP markers and primers in the QTL and $\mathrm{mQTL}$ region on chromosome 6. Primers M11 and Isotig 18067-441 were used for plant selection. HmpsE088 and HmpsE113 flanked the previously detected mQTLs that show overlap with the previously defined QTL region (Maharijaya et al., 2015; Maharijaya et al., 2019).

\begin{tabular}{|c|c|c|c|}
\hline Marker ID & Position Zunla & $\begin{array}{l}\text { Forward primer } \\
\left(5^{\prime}-3^{\prime}\right)\end{array}$ & $\begin{array}{l}\text { Reverse primer } \\
\left(5^{\prime}-3^{\prime}\right)\end{array}$ \\
\hline$M 11^{1}$ & $24.2 \mathrm{Mbp}$ & $\begin{array}{l}\text { ATCTTCGATGGGTA } \\
\text { AATGCTTG }\end{array}$ & $\begin{array}{l}\text { GGGCTTAACGTIT } \\
\text { ACTGAGATCC }\end{array}$ \\
\hline Isotig $18067-441^{2}$ & $25.1 \mathrm{Mbp}$ & $\begin{array}{l}\text { GGTCTGGCTGGTCT } \\
\text { AGT }\end{array}$ & $\begin{array}{l}\text { GAGAGAGGGACATG } \\
\text { ATCGT }\end{array}$ \\
\hline $\mathrm{HmpsE088}^{3}$ & $5.8 \mathrm{Mbp}$ & $\begin{array}{l}\text { CCAAACGAACCGAT } \\
\text { GAACACTC }\end{array}$ & $\begin{array}{l}\text { GACAATGTTGAAAA } \\
\text { AGGTGGAAGAC }\end{array}$ \\
\hline HmpsE113 3 & 43.1 Mbp & $\begin{array}{l}\text { CCCTAAAGCTCGAG } \\
\text { AAATTGAAGC }\end{array}$ & $\begin{array}{l}\text { GAATGCTGTTGCTG } \\
\text { GGGTTGTT }\end{array}$ \\
\hline
\end{tabular}

\section{Thrips rearing and synchronization}

A population of Frankliniella occidentalis (Pergande) that originated from Greenhouse Horticulture of Wageningen University and Research, Bleiswijk, The Netherlands, was used to phenotype the plants for thrips resistance. $F$. occidentalis was reared in glass jars covered with a thrips proof gauze, in a growth cabinet at $25^{\circ} \mathrm{C}, \mathrm{L} 16: \mathrm{D} 8,70 \% \mathrm{RH}$. The thrips were fed on Phaseolus vulgaris beans. Synchronized first instar larvae (L1) were obtained by allowing female adults to lay eggs on snack cucumber for 24 hours, after which they were carefully removed by using a brush. The snack cucumbers were kept in a growth cabinet at $25^{\circ} \mathrm{C}$. After 4 days, synchronized neonate first instar larvae were obtained. 


\section{Detached leaf assay}

The level of thrips resistance in the selected pepper F4 plants as well as the resistant and susceptible references was determined at a plant age of 12 weeks in a detached leaf assay, as previously described by Van Haperen et al. (2019) (Chapter 2). The seven youngest fully opened leaves per plant were collected. Each detached leaf was placed with the petiole in a droplet of $1.5 \%$ water agar in a Petri dish (BD Falcon, tight-fit lid $50 \times 9 \mathrm{~mm}$ ).

Three leaves were used to determine development of first instar (L1) into second instar larvae (L2). Five synchronized first instar larvae were placed on these leaves. The Petri dishes were stored in a growth cabinet at $25^{\circ} \mathrm{C}$. The developmental stage was determined at 3, 5 and 7 days after infestation. The fraction L1 that did not develop into the next larval stage was used as a measure of thrips resistance. A high fraction L1 means that the first instar larvae did not develop into the next larval stage, and thus indicates resistance. The development of L1 into L2 occurs in 1-2 days under optimal conditions (Lublinkhof and Foster, 1977).

The other four leaves of the F4 plants were used to obtain leaf material for RNAsequencing (Chapter 3 ) and metabolite extraction. Four leaves of each plant that was included as resistant or susceptible reference were obtained to extract metabolites as well. Fifteen synchronized L1s were placed on two of the four leaves (hereafter referred to as "thrips-exposed"). The other two leaves were not infested ("mock-treated"). After six hours, all four leaves per plant were frozen in liquid nitrogen and stored in $-80^{\circ} \mathrm{C}$ until further use.

\section{Metabolite extraction and profiling using LC-MS}

Every sample consisted of a pool of ten leaves, either infested or mock-treated, taken from five plants of the same QTL genotype, i.e. with either the "R" allele or the " $S$ " allele in homozygous state, or from the resistant or susceptible reference. The leaves were ground into a fine powder in liquid nitrogen and $300 \mathrm{mg}$ fresh weight of leaf powder per sample was used to extract their metabolites. Two technical quality control samples (TC), consisting of mixed powder from different samples, were included. One of the pooled samples of group $R$, mock-treated, dropped out due to a technical problem of the LC-MS. Metabolites were extracted with $900 \mu \mathrm{L} 99.87 \% \mathrm{MeOH}$ containing $0.13 \%$ formic acid. LC-MS was performed using an HPLC system (Waters Acquity) coupled to an LTQ Orbitrap FTMS hybrid mass spectrometer (Thermo Scientific) using a Phenomena Luna C18 column and a gradient of water and acetonitrile. Negative electrospray mode was used at a mass resolution of 60,000 (full width at half maximum) and a mass range of $\mathrm{m} / \mathrm{z}$ 90-1350 D. Metalign software (Lommen, 2009) was used to pick mass peaks in 
an unbiased manner. Mass peaks that were predicted to belong to the same metabolite were determined by MSClust software (Tikunov et al., 2012). From here on, mass peak clusters will be referred to as 'metabolites'.

\section{Data analysis}

In the metabolite data, non-detects were randomized with values between 45000 and 55000 , as this was 45 to $55 \%$ of the local chromatographic noise as calculated by the Metalign software. The data was transformed as $y=\log 10(x)$ and t-tests were carried out in Excel to determine significant differences in metabolites between different groups. False discovery rate correction (FDR) with $a=0.10$ was used to correct for multiple comparisons (Benjamini and Hochberg, 1995). A principal components analysis (PCA) was carried out using SIMCA version 15.02 (Umetrics, Umea, Sweden). Data were normalized across samples with Pareto scaling before carrying out the PCA analysis. As one of the three thrips-exposed samples from group $S$ clearly deviated from the two other biological replicates, this sample was excluded from the analysis.

To find the genes in the QTL region that might play a role in diterpene glycoside biosynthesis, the genome annotation Capsicum annuum Zunla-1 from the Pepper genome database (Release 2.0; http://public.genomics.org.cn/BGI/pepper/) was used. BLAST was used to determine the physical position of two markers flanking the region where six mQTLs that co-localized with the resistance QTL were located on the Zunla-1 assembly (Qin et al., 2014; Maharijaya et al., 2019) (HmpsE088 and HmpsE113; Table 1).

\section{Results}

\section{Larval development in resistant and susceptible F4 plants}

We observed a significant difference in fraction first instar larvae (L1) between the $\mathrm{R}$ and $\mathrm{S}$ group (Figure 1 ). Also, we observed a significant difference between the resistant (CGN16975A) and susceptible (CGN17219A) reference. A high fraction $L 1$ indicates inhibition of larval development, thus resistance. These results indicate that these plant materials are highly contrasting in their resistance levels and thus may have differentially accumulated metabolites linked to their thrips resistance level. 


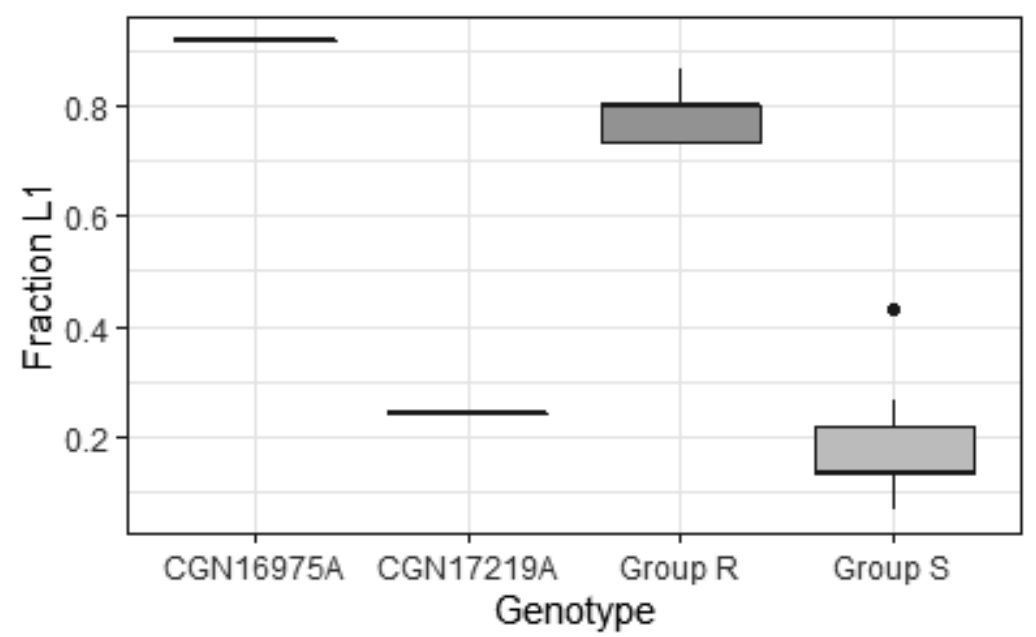

Figure 1: Larval development in Capsicum plants selected for metabolomics. The fraction L1 larvae was determined in plants that had the resistance allele (group R) or susceptibility allele (group S) in homozygous state for the fine-mapped QTL region. A high fraction L1 refers to inhibition of larval development, which was used as a measure for thrips resistance. A significant difference in fraction L1 was found between group R and group $S$, and between the resistant (CGN16975A) and susceptible (CGN17219A) reference ( $<$ < $0.001, n=15)$.

\section{Metabolomics}

Metabolites were extracted from leaves of plants from both the $\mathrm{R}$ and $\mathrm{S}$ group, and the resistant and susceptible references, both exposed to L1 and mocktreated leaves. In negative ionization mode a total of 1363 putative metabolites were detected. Forty of these metabolites contain an in-source fragment of $\mathrm{m} / \mathrm{z}$ 271.24, which is a common fragment of diterpene glycosides (Heiling et al., 2016). Thirty-nine of these compounds are present in all tested samples. One of the putative diterpene glycosides is only detected in the resistant accession, in both thrips-exposed and mock-treated plants.

The PCA plot based on metabolite profiles of all samples, i.e. both group R, group $S$ and the resistant (CGN16975A) and susceptible (CGN17219A) reference, shows a separation of plants into 3 different clusters (Figure 2). A clear separation between the resistant and susceptible references is observed, resulting in two distinct clusters of samples. The third cluster of samples contains the leaf extracts of both group R and group S F4 plants. We did not observe a clear separation between mock-treated and thrips- exposed samples, neither within the clusters for the resistant and susceptible references, nor within the group $\mathrm{R}$ and group $\mathrm{S}$ cluster (Figure 3). 


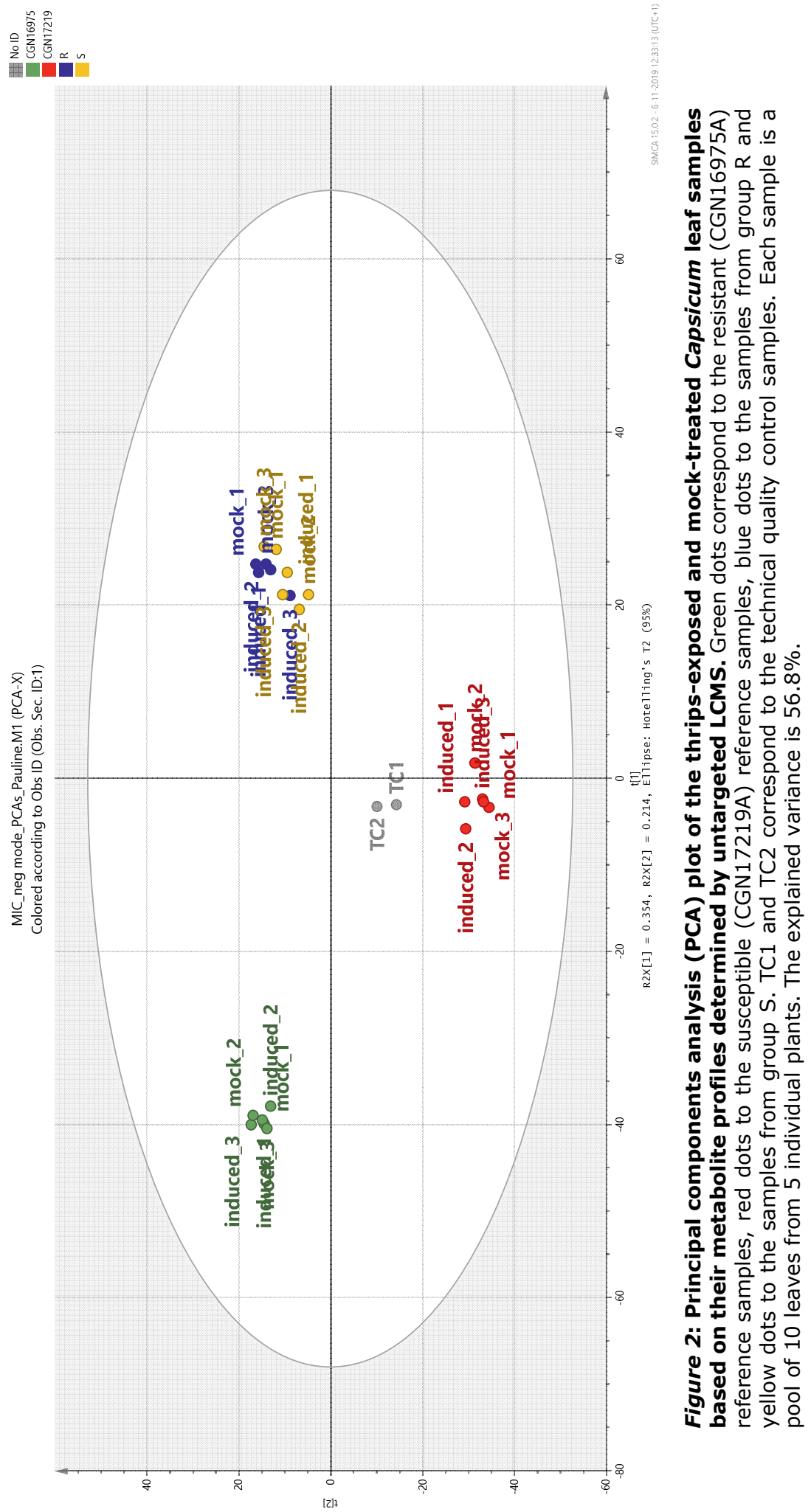


ㅇ

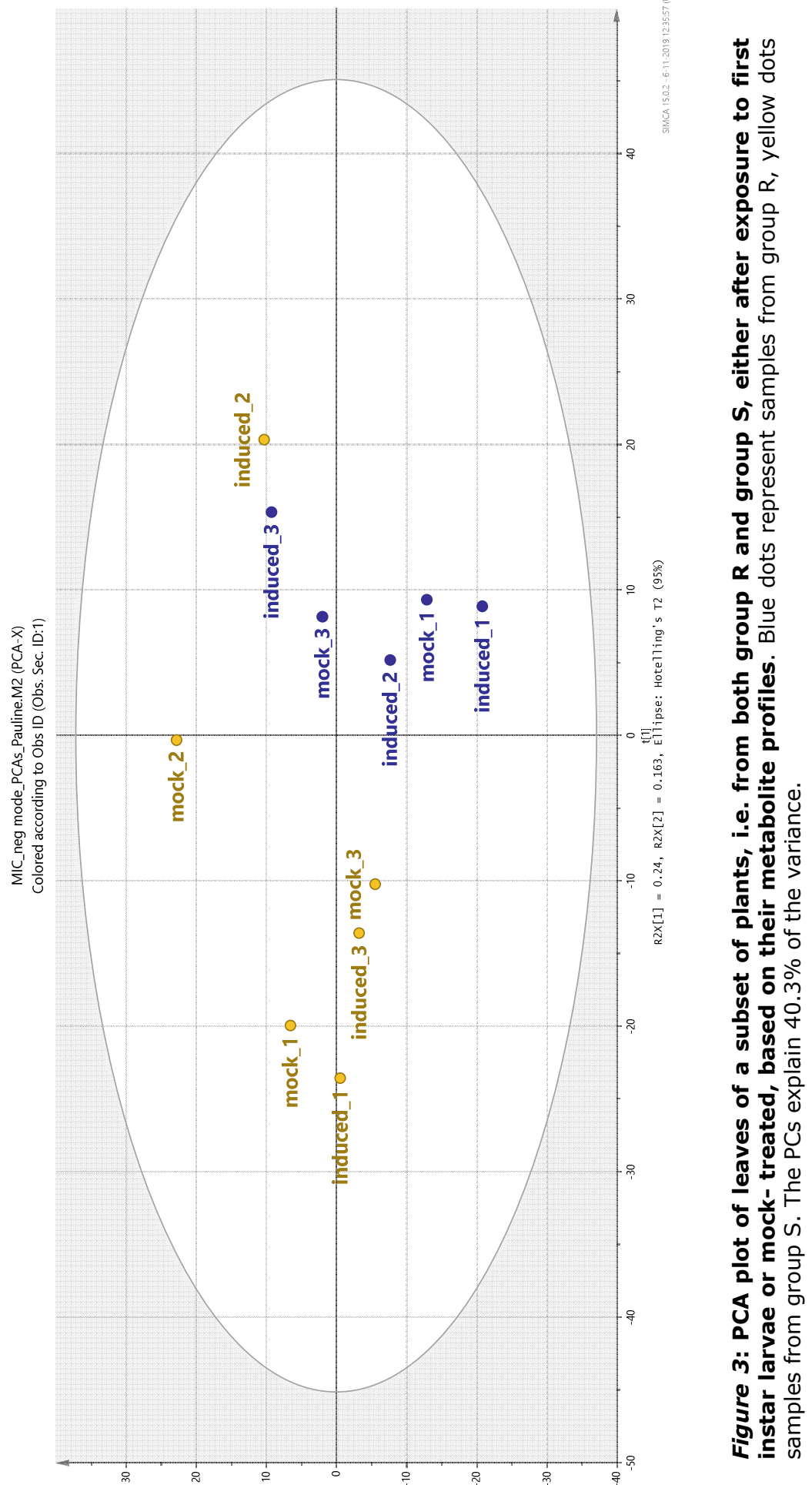


After correcting for multiple testing, 856 metabolites were found to differ significantly in relative intensity between the resistant and susceptible references for both treatments (Figure 4A; Supplementary data Table S2). Thirty-six of these metabolites are putative diterpene glycosides, of which 18 were more abundant in the resistant, and the other 18 more abundant in the susceptible reference accession. When considering the effect of thrips feeding, we found that within the susceptible reference, only two metabolites were significantly different between thrips-exposed and mock-treated samples, while only one metabolite was significantly different within the resistant reference (Supplementary data Table S3). Between group R and S of the F4 lines, 32 metabolites differed significantly in their relative intensity for both treatments (Figure 4B; Supplementary data Table S4). For group $\mathrm{R}$, two metabolites differed significantly in relative intensity between the thrips-exposed and mock-treated groups (Supplementary data Table S5). For group S, four such metabolites were observed. However, none of putative compounds that significantly differed between group $\mathrm{R}$ and group $\mathrm{S}$, or between thrips-exposed and mock-treated within group $\mathrm{R}$ and $\mathrm{S}$ respectively, could be annotated from their clustered mass signals. The most likely explanation for this is that these represented artefacts resulting from false-positive signals picked up by the unbiased processing procedure, which can be due to e.g. local matrixdependent differences in ion suppression of the column eluent (Antignac et al., 2005). None of the mass cluster peaks that differed significantly between thripsexposed and mock-treated leaf samples contained the $\mathrm{m} / \mathrm{z} 271,24$ fragment that is characteristic for diterpene glycosides.
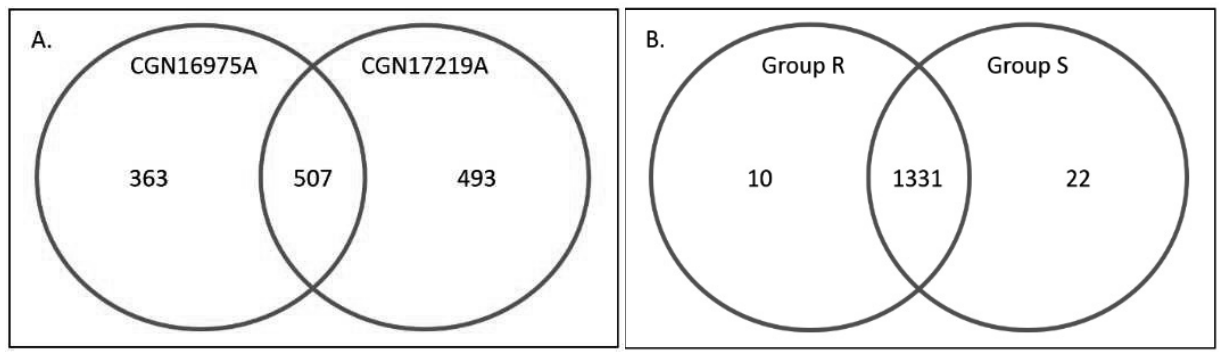

Figure 4: Overview of the number of differentially accumulated metabolites between the resistant (CGN16975A) and susceptible (CGN17219A) references (panel $A$ ), and between group $R$ and group $S$ (panel $B$ ) in negative mode. Numbers indicate the number of metabolites that are significantly more abundant in relative intensity in one of the two plant groups ( $P$ adjusted $<0.05$ ), or that did not significantly differ between these two groups (numbers in common between group circles). These numbers include some artefacts, including the 10 and 22 in panel B. 


\section{Diterpene biosynthesis genes within the resistance-QTL}

The flanking markers of the mQTLs that co-localize with the resistance QTL as defined by Maharijaya et al. (2015) (Table 1) were mapped to the Zunla-1 assembly at physical positions 5.77 Mbp and 43.14 Mbp (Maharijaya et al., 2019). Between these flanking markers, 812 genes were predicted according to the Pepper genome database. Twenty-one of these 812 genes putatively encode enzymes that may be involved in the diterpenoid biosynthesis pathway, of which 15 genes encode a cytochrome P450 (Supplementary Table S6) and six genes encode, respectively, a geranylgeranyl transferase subunit, three gibberellin 20 oxidases, and two gibberellin 3-beta-dioxygenases. Only one of the genes, Capana06g001248 putatively encoding cytochrome P450 94A2, is located in the fine-mapped QTL region (24.43-24.81Mbp; Chapter 3).

\section{Discussion}

In this study, we compared plants within one F4 line that either had the thrips resistance or susceptibility allele in homozygous state for the fine-mapped QTL region on chromosome 6 , and leaves exposed to first instar thrips (L1) or mocktreated. We performed an untargeted LC-MS analysis of water-methanol extractable metabolites, including capsianosides previously reported to be associated with thrips resistance, on young leaves of 12 week old plants in which the effect of the resistance QTL was clearly detectable. No significant differences in the relative abundances of the detected metabolites were found between the two contrasting groups of F4 plants, neither between L1-exposed and mocktreated samples within each sample group, as the detected mass peaks that significantly differ between the treatments are most likely artefacts. This observation suggests that the metabolites that could be detected using our extraction and LC-MS method do not play a role in the resistance mechanism. However, we cannot exclude the possibility that metabolites do play a role in resistance, but the diterpene glycosides detectable with our method do not. This is an unexpected result, as these metabolites were previously shown to be associated with thrips resistance in Capsicum (Macel et al., 2019; Maharijaya et al., 2019). Also, we expected to observe higher levels of diterpene glycosides upon exposure to thrips, as thrips feeding induces a JA-related defence in plants (Abe et al., 2009; Sarde et al., 2019), and levels of diterpene glycoside increase upon JA-application in Nicotiana attenuata (Keinänen et al., 2001; Heiling et al., 2010). We detected 40 putative diterpene glycosides of which 36 significantly differed between the resistant and susceptible references. One of these putative diterpene glycosides was only present in the resistant reference, but neither in the susceptible reference nor in any of the F4 plants. The outcome of this metabolomics analysis shows that, although we observed differences in putative 
diterpene glycosides between the resistant and susceptible reference, these diterpene glycosides do not play a key role in the resistance mechanism encoded by genes located in the QTL on chromosome 6.

Twenty-one genes that might play a role in diterpene glycoside synthesis were found in the previously defined QTL region on chromosome 6. Fifteen of the 21 genes encode cytochrome P450 (CYP), which might play a role in the final steps of the diterpene glycoside pathway. As each CYP catalyses a different reaction, it is unlikely that all CYPs play a role in the diterpene glycoside synthesis. Four CYP families, from which seven members were found in the $\mathrm{mQTL}$ region, were previously described to play a potential role in terpenoid metabolism (Christoffersen Rolf et al., 1995; Schopfer and Ebel, 1998; Ro et al., 2005; Ohnishi et al., 2006; Thornton et al., 2010; Höfer et al., 2013). The CYP89 and CYP94 families, from which eight members were found in the MQTL region, do not seem to play a role in the terpenoid pathway (Kahn et al., 2001; Christ et al., 2013). The six other genes encode geranylgeranyltransferase type-1 subunit alpha, two gibberellin 20 oxidase 1, gibberellin oxidase 3, and gibberellin 3-beta-dioxygenase 1 and 3. Geranylgeranyltransferase type-1 subunit alpha is an essential subunit of the geranylgeranyltransferase complex, which plays a role in the transfer of the precursor of diterpenes, i.e. geranylgeranyl-diphosphate, to the cysteine residue of a protein (Yalovsky et al., 1997). Gibberellin 20 oxidase 1 and 3, and gibberellin 3-beta-dioxygenase are involved in the biosynthesis and activation of gibberellin, which belong to a large family of diterpenoid plant hormones (Williams et al., 1998; Rieu et al., 2008; Zi et al., 2014). However, all these genes, except cytochrome P450 94A2, are located outside of the fine-mapped QTL region conferring thrips resistance, as described in Chapter 3. Maharijaya et al. (2019) identified mQTLs that showed overlap with the resistance QTL on chromosome 6. Therefore, it is conceivable that the genes located in these mQTLs play a role in diterpene glycoside synthesis, but that diterpene glycosides themselves do not play a key role in the differential thrips resistance controlled by genes located in the fine-mapped QTL. This suggestion is supported by the metabolomics analysis, as we did observe significant difference in thrips resistance levels between group $\mathrm{R}$ and $\mathrm{S}$, but did not detect significant differences in abundance of identified diterpene glycosides nor in any other detected metabolite.

We can conclude that the resistance mechanism underlying this specific finemapped QTL most likely works independently from the diterpene glycoside pathway. Using the same analytical platform, it was shown that diterpene glycosides are correlated with thrips resistance in Capsicum (Macel et al., 2019; Maharijaya et al., 2019). However, the role of diterpene glycoside abundance in thrips resistance in Capsicum needs to be re-evaluated, as it is not proven that 
diterpene glycosides cause thrips resistance. The metabolome is the end result of many cellular processes, thus the plant's ultimate response to genetic and environmental factors. Studying the metabolome is an exploratory tool that needs validation of observed correlations with traits before conclusions about the underlying mechanisms can be drawn (Fiehn, 2002; Camacho et al., 2005). Due to the complex connection between metabolites of seemingly unrelated pathways, for instance due to pleiotropic effects, a correlation between metabolites as end products and resistance might not lead to identifying the causal pathway or gene (Fiehn, 2002). In order to confirm that diterpene glycosides do not play a role in thrips resistance in Capsicum, we suggest to knock-out genes that play a role in diterpene glycoside biosynthesis in resistant Capsicum accessions, and determine whether or not this knock-out affected the level of thrips resistance.

\section{Acknowledgement}

The authors thank Betty Henken, Mengjing Sun and Wendy van 't Westende for carrying out the detached leaf assay ( $\mathrm{BH}, \mathrm{MS}$ and WvtW), and for carrying out the metabolite extraction (WvtW). We thank Bert Schipper for operating the LCMS. This research was supported by a grant (TKI-1409-045) from the Ministry of Economic Affairs of The Netherlands, and by breeding companies Nunhems Netherlands B.V. and Bejo Zaden B.V.

\section{Author contributions}

$\mathrm{PvH}, \mathrm{RV}, \mathrm{JVL}$ and $\mathrm{BV}$ conceived and designed the experiments. HvE performed the metabolite extractions, and $\mathrm{HvE}$ and RdV processed and analysed the metabolite data. PvH carried out the experimental work, data analysis and wrote the first draft of the manuscript. PvH, RV, JvL, RdV and BV were involved in revision of the manuscript. 


\section{Supplementary data}

Table S1A and B: Enzymes involved in the synthesis of the diterpenoid precursor geranylgeranyl pyrophosphate (GGPP) in the methylerythritol 4-phosphate (MEP) pathway $(A)$ and the enzymes that use the precursor to synthesize diterpenoids (B). This overview is based on the C5 isoprenoid biosynthesis, non-mevalonate pathway and diterpenoid synthesis pathway as described at the KEGG database entry M00096 and M00904 (Walker et al., 2002; Long and Croteau, 2005).

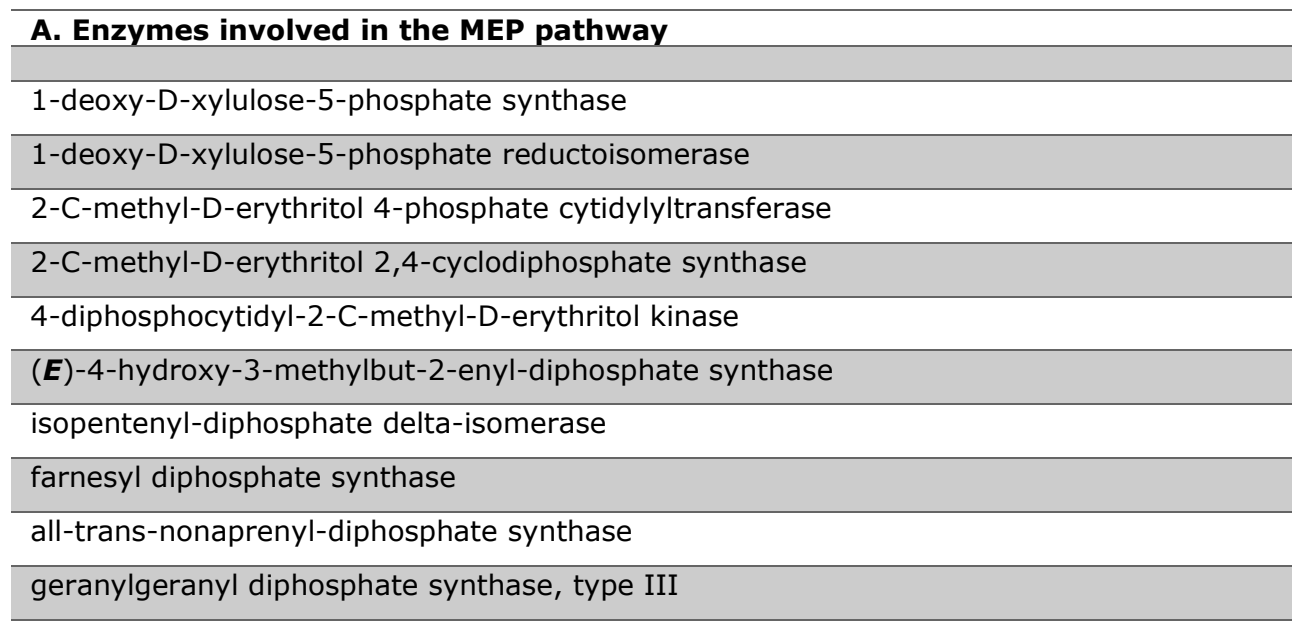

B. Enzymes involved in the diterpenoid synthesis pathway ent-copalyl diphosphate synthase

\begin{tabular}{l}
\hline ent-kaurene synthase \\
\hline ent-kaurene oxidase \\
\hline ent-kaurenoic acid monooxygenase \\
\hline gibberellin 20 oxidase 1-like \\
\hline gibberellin 2-beta-dioxygenase
\end{tabular}

Table S2: Clusters of mass peaks, i.e. putative metabolites, that show a significant difference in abundance between the resistant and susceptible reference. These clusters were significant after correction for multiple comparison (FDR=0.10).

Please find the Supplementary data Table S2 through the following link: https://drive.google.com/open?id=1fZ0NWiXJLS3fMBenjuuU G0ONGIbdpVf 
Table S3: Metabolites (mass cluster ID) that showed a significant difference in relative abundance between thrips-exposed and mock-treated leaf samples. Metabolites showed a significant difference after correction for multiple comparison $(F D R=0.10)$. Columns indicated with "Resistant reference" and "Susceptible reference" indicate the $\log 10$ of the relative abundance of thrips-exposed and mock-treated samples.

\begin{tabular}{|c|c|c|c|c|c|c|c|}
\hline \multirow{3}{*}{$\begin{array}{l}\text { Cluster } \\
\text { ID }\end{array}$} & \multirow{3}{*}{$\begin{array}{l}\text { \#peaks in } \\
\text { cluster }\end{array}$} & \multirow{3}{*}{$\begin{array}{l}\text { Retention } \\
\text { time (min) }\end{array}$} & \multirow{2}{*}{\multicolumn{2}{|c|}{ Resistant reference }} & \multirow{2}{*}{\multicolumn{2}{|c|}{$\begin{array}{l}\text { Susceptible } \\
\text { reference }\end{array}$}} & \multirow[t]{3}{*}{$P$-value } \\
\hline & & & & & & & \\
\hline & & & $\begin{array}{l}\text { Mock- } \\
\text { treated }\end{array}$ & $\begin{array}{l}\text { Thrips- } \\
\text { exposed }\end{array}$ & $\begin{array}{l}\text { Mock- } \\
\text { treated }\end{array}$ & $\begin{array}{l}\text { Thrips- } \\
\text { exposed }\end{array}$ & \\
\hline $313^{1}$ & 3 & 7.47 & 4.99 & 5.04 & 5.54 & 4.67 & $<0.001$ \\
\hline $2057^{1}$ & 3 & 39.31 & 4.69 & 4.77 & 5.63 & 4.68 & $<0.001$ \\
\hline $2226^{2}$ & 16 & 45.66 & 4.70 & 4.67 & 4.68 & 4.78 & $<0.001$ \\
\hline
\end{tabular}

1 indicates clusters that showed significant differences between thrips-exposed and mock-treated samples of the susceptible reference.

2 indicates the cluster that significantly differed in relative abundance between thripsexposed and mock- treated samples of the resistant reference. 
Table S4: Metabolites (mass cluster ID) that showed a significant difference in relative abundance between leaf samples from group $\mathbf{R}$ and group $\mathbf{S}$. Significance of differences was determined after correction for multiple comparison (FDR=0.10). Columns indicated with "Group R" and "Group S" indicate the log10 of the relative abundance of thrips-exposed and mock-treated samples.

\begin{tabular}{|c|c|c|c|c|c|c|c|}
\hline \multirow{2}{*}{$\begin{array}{l}\text { Cluster } \\
\text { ID }\end{array}$} & \multirow{2}{*}{$\begin{array}{l}\text { \#peaks } \\
\text { in } \\
\text { cluster }\end{array}$} & \multirow{2}{*}{$\begin{array}{l}\text { Retention } \\
\text { time } \\
\text { (min) }\end{array}$} & \multicolumn{2}{|l|}{ Group R } & \multicolumn{2}{|l|}{ Group S } & \multirow[t]{2}{*}{ P-value } \\
\hline & & & $\begin{array}{l}\text { Mock- } \\
\text { treated }\end{array}$ & $\begin{array}{l}\text { Thrips- } \\
\text { exposed }\end{array}$ & $\begin{array}{l}\text { Mock- } \\
\text { treated }\end{array}$ & $\begin{array}{l}\text { Thrips- } \\
\text { exposed }\end{array}$ & \\
\hline 2072 & 21 & 39.89 & 7.93 & 7.73 & 5.04 & 4.66 & $<0.001$ \\
\hline 319 & 6 & 8.11 & 4.68 & 4.78 & 5.55 & 5.70 & $<0.001$ \\
\hline 326 & 2 & 8.44 & 4.67 & 4.67 & 5.03 & 5.13 & $<0.001$ \\
\hline 2115 & 6 & 41.34 & 4.86 & 4.68 & 5.42 & 5.36 & $<0.001$ \\
\hline 333 & 5 & 9.01 & 5.06 & 4.94 & 5.75 & 5.83 & $<0.001$ \\
\hline 321 & 5 & 8.32 & 4.77 & 4.83 & 5.83 & 6.02 & $<0.001$ \\
\hline 344 & 8 & 9.39 & 5.30 & 5.13 & 6.20 & 6.33 & $<0.001$ \\
\hline 317 & 9 & 8.05 & 5.76 & 5.89 & 6.96 & 7.12 & $<0.001$ \\
\hline 315 & 8 & 7.74 & 5.60 & 5.92 & 6.80 & 6.94 & $<0.001$ \\
\hline 309 & 8 & 7.51 & 5.01 & 5.00 & 5.56 & 5.62 & $<0.001$ \\
\hline 297 & 5 & 6.82 & 4.67 & 4.67 & 5.49 & 5.62 & $<0.001$ \\
\hline 314 & 9 & 7.71 & 4.81 & 4.98 & 5.53 & 5.69 & $<0.001$ \\
\hline 2146 & 7 & 42.46 & 6.23 & 6.64 & 4.70 & 4.69 & $<0.001$ \\
\hline 329 & 7 & 8.67 & 5.57 & 5.72 & 6.76 & 6.84 & $<0.001$ \\
\hline 302 & 11 & 7.02 & 4.99 & 4.96 & 5.68 & 6.09 & $<0.001$ \\
\hline 303 & 11 & 7.05 & 5.13 & 5.46 & 6.59 & 6.81 & $<0.001$ \\
\hline 68 & 95 & 1.96 & 8.05 & 8.07 & 7.98 & 8.00 & $<0.001$ \\
\hline 2027 & 6 & 38.43 & 5.72 & 5.67 & 5.91 & 5.81 & $<0.001$ \\
\hline 2166 & 4 & 43.20 & 5.13 & 5.34 & 4.74 & 4.69 & $<0.001$ \\
\hline 843 & 37 & 18.85 & 7.53 & 7.53 & 7.30 & 7.28 & $<0.001$ \\
\hline 981 & 45 & 20.80 & 5.33 & 5.28 & 5.51 & 5.46 & 0.001 \\
\hline 951 & 43 & 20.36 & 6.23 & 6.31 & 6.13 & 6.11 & 0.001 \\
\hline 284 & 8 & 6.14 & 4.69 & 4.71 & 5.98 & 5.93 & 0.001 \\
\hline 2174 & 3 & 43.38 & 5.21 & 5.42 & 4.77 & 4.75 & 0.001 \\
\hline 287 & 9 & 6.32 & 4.71 & 4.67 & 5.46 & 5.69 & 0.001 \\
\hline 335 & 7 & 9.12 & 5.31 & 5.62 & 6.62 & 6.71 & 0.001 \\
\hline 328 & 9 & 8.67 & 5.59 & 5.78 & 6.86 & 6.99 & 0.001 \\
\hline 2129 & 9 & 42.09 & 5.94 & 6.75 & 4.68 & 4.78 & 0.001 \\
\hline 2011 & 2 & 37.90 & 5.43 & 5.17 & 4.67 & 4.72 & 0.002 \\
\hline 62 & 120 & 1.90 & 7.27 & 7.29 & 7.17 & 7.18 & 0.002 \\
\hline 355 & 7 & 9.87 & 5.20 & 5.32 & 6.33 & 6.43 & 0.002 \\
\hline 357 & 4 & 10.08 & 5.03 & 4.87 & 5.84 & 5.82 & 0.002 \\
\hline
\end{tabular}


Table S5: Metabolites (mass cluster ID) that showed significant difference in relative abundance between thrips-exposed and mock-treated leaf samples in F4 plants. Metabolites were identified as significantly different after correction for multiple comparison (FDR=0.10). Columns indicated with "Group R" and "Group S" indicate the $\log 10$ of the relative abundance of thrips-exposed and mock-treated samples.

\begin{tabular}{|c|c|c|c|c|c|c|c|}
\hline \multirow{2}{*}{$\begin{array}{l}\text { Cluster } \\
\text { ID }\end{array}$} & \multirow{2}{*}{$\begin{array}{l}\text { \#peaks } \\
\text { in } \\
\text { cluster }\end{array}$} & \multirow{2}{*}{$\begin{array}{l}\text { Retention } \\
\text { time } \\
\text { (min) }\end{array}$} & \multicolumn{2}{|l|}{ Group R } & \multicolumn{3}{|l|}{ Group S } \\
\hline & & & $\begin{array}{l}\text { Mock- } \\
\text { treated }\end{array}$ & $\begin{array}{l}\text { Thrips- } \\
\text { exposed }\end{array}$ & $\begin{array}{l}\text { Mock- } \\
\text { treated }\end{array}$ & $\begin{array}{l}\text { Thrips- } \\
\text { exposed }\end{array}$ & P-value \\
\hline $585^{1}$ & 9 & 14.97 & 5.58 & 4.83 & 5.70 & 4.66 & $<0.001$ \\
\hline $2040^{1}$ & 21 & 38.75 & 6.81 & 6.42 & 6.92 & 6.14 & $<0.001$ \\
\hline $1920^{1}$ & 10 & 35.31 & 6.50 & 6.04 & 6.62 & 5.83 & $<0.001$ \\
\hline $2085^{1}$ & 7 & 40.20 & 5.72 & 4.70 & 5.93 & 4.73 & $<0.001$ \\
\hline $1974^{2}$ & 2 & 36.62 & 5.24 & 4.66 & 5.44 & 5.53 & $<0.001$ \\
\hline $2098^{2}$ & 3 & 40.63 & 5.16 & 4.66 & 5.16 & 4.71 & $<0.001$ \\
\hline
\end{tabular}

${ }^{1}$ indicates metabolites that showed significant differences between thrips-exposed and mock-treated samples of Group S

2 indicates the metabolites that significantly differed in relative abundance between thripsexposed and mock-treated samples of Group R.

Table S6: Overview of the genes in the QTL region on chromosome 6 that encode enzymes which are possibly involved in the diterpenoid biosynthesis pathway

\begin{tabular}{|c|c|c|}
\hline Zunla gene ID & $\begin{array}{l}\text { Gene } \\
\text { start }\end{array}$ & Description \\
\hline Capana06g000416 & 6,12 & cytochrome P450 71A9 \\
\hline Capana06g000592 & 9,10 & cytochrome P450 94A1 \\
\hline Capana06g000623 & 9,57 & cytochrome P450 89A9 \\
\hline Capana06g000624 & 9,57 & cytochrome P450 89A9 \\
\hline Capana06g000715 & 11,33 & $\begin{array}{l}\text { Protein farnesyltransferase/geranylgeranyltransferase type-1 } \\
\text { subunit alpha }\end{array}$ \\
\hline Capana06g000908 & 15,76 & cytochrome P450 94A1 \\
\hline Capana06g000910 & 15,82 & cytochrome P450 94A2 \\
\hline Capana06g000911 & 15,82 & cytochrome P450 94A1 \\
\hline Capana06g001151 & 20,80 & cytochrome P450 734A1 \\
\hline Capana06g001248 & 24,31 & cytochrome P450 94A2 \\
\hline Capana06g001291 & 27,47 & Gibberellin 20 oxidase 1 \\
\hline Capana06g001292 & 27,49 & Gibberellin 3-beta-dioxygenase 3 \\
\hline Capana06g001294 & 27,51 & Gibberellin 20 oxidase 1 \\
\hline Capana06g001302 & 27,71 & Gibberellin 20 oxidase 3 \\
\hline Capana06g001303 & 27,73 & Gibberellin 3-beta-dioxygenase 1 \\
\hline Capana06g001389 & 30,71 & cytochrome P450 716B2 \\
\hline Capana06g001391 & 30,73 & cytochrome P450 716B2 \\
\hline Capana06g001470 & 33,48 & cytochrome P450 94A2 \\
\hline Capana06g001487 & 34,77 & cytochrome P450 76C4 \\
\hline Capana06g001491 & 34,85 & cytochrome P450 76C2 \\
\hline Capana06g001493 & 34,87 & cytochrome P450 76C4 \\
\hline
\end{tabular}





\section{CHAPTER 5}
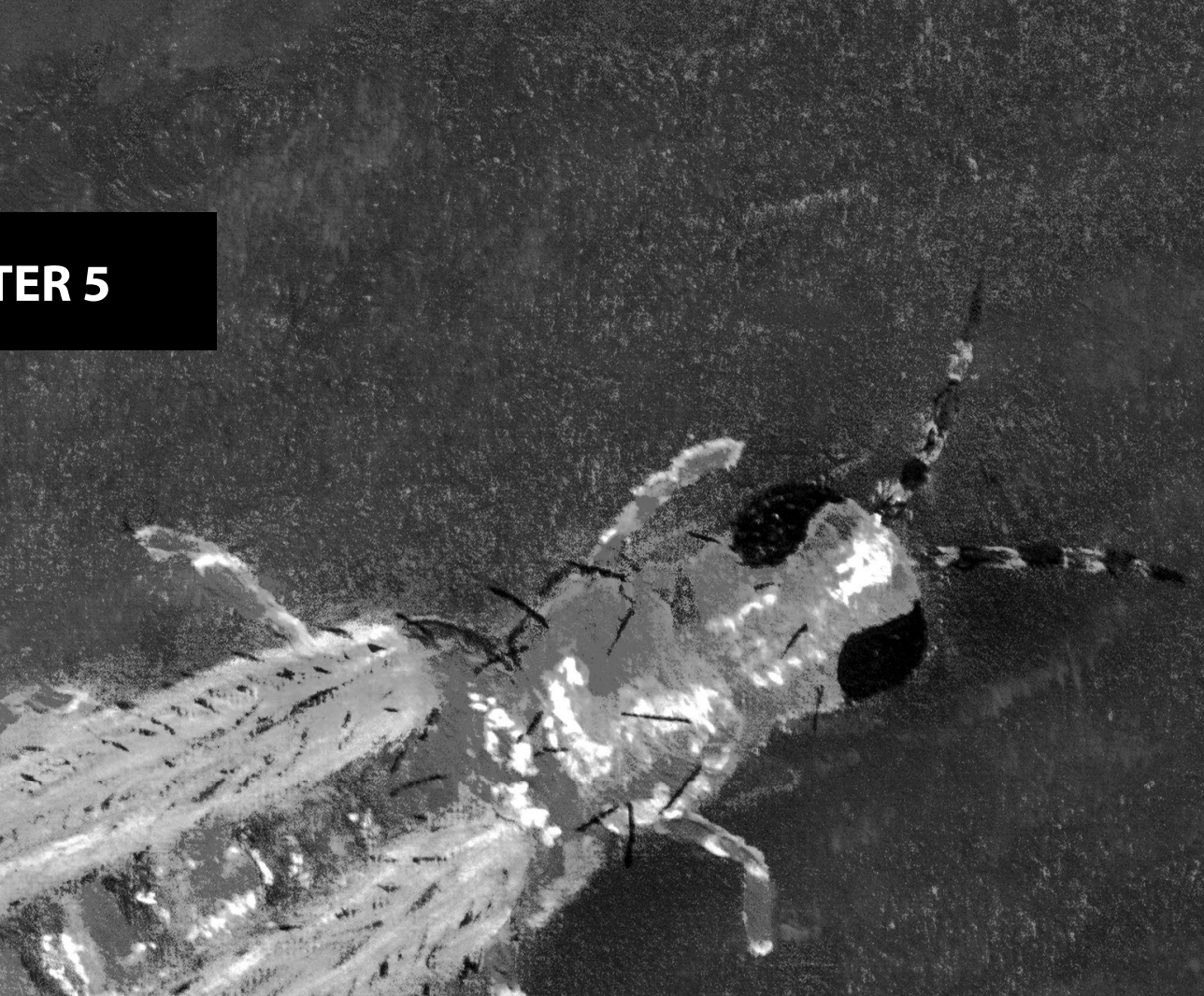

$\operatorname{lin}(1)$

(t)

14.

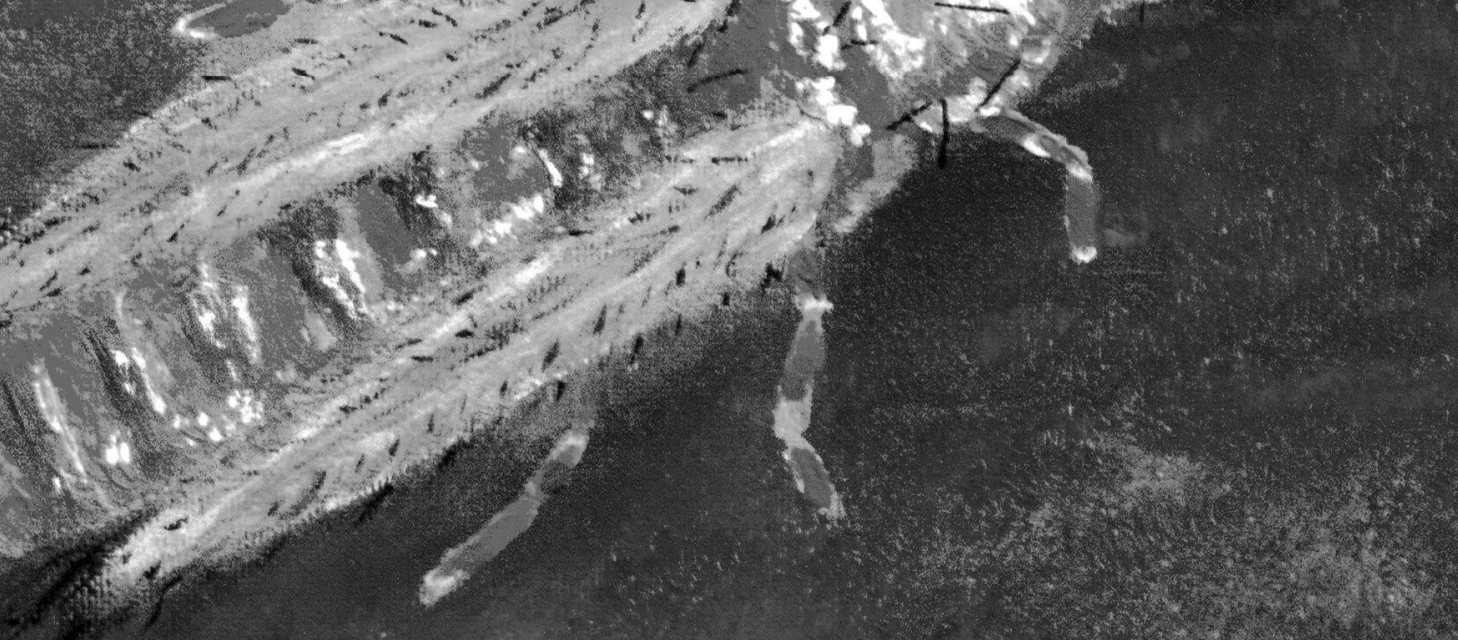

$-3+2 x^{3}$
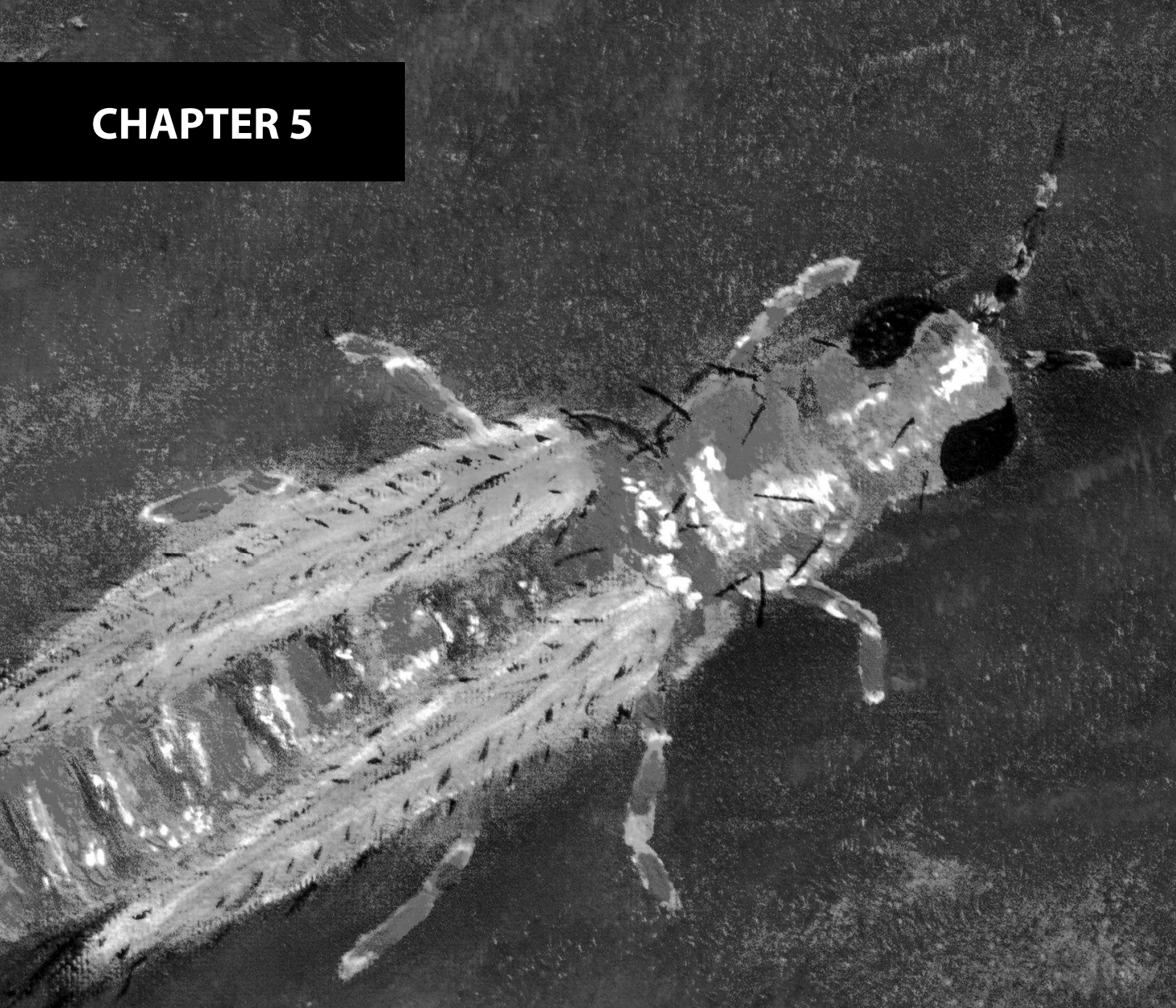

$4 \times 2.85$
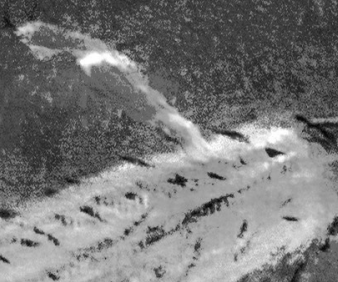

$1 \geq$

1.) 


\section{The effect of a thrips resistance QTL in different genetic backgrounds}

Pauline van Haperen, Roeland E. Voorrips, Alejandro F. Lucatti,

Wijnand Schellart, Joop J.A. van Loon, Ben Vosman 


\begin{abstract}
Thrips are a worldwide threat to Capsicum because they damage fruits, flowers and leaves directly by feeding, and indirectly by vectoring tospoviruses such as tomato spotted wilt virus. Therefore, growers would benefit from thrips-resistant varieties. Previously, a QTL that provides resistance to Frankliniella occidentalis has been identified. Here we explore the potential of this QTL for breeding thripsresistant varieties by studying its effect on two thrips species (i.e. F. occidentalis and Thrips tabaci) in four different Capsicum backgrounds. We observed differences in thrips resistance between different genetic backgrounds, both in plants that have the resistance allele in homozygous state as well as in plants with the susceptibility allele in homozygous state for the QTL region. This finding suggests the presence of factors in these backgrounds that either increase or reduce thrips resistance. Altogether, we confirmed the major effect of the QTL on thrips resistance in all four genetic backgrounds to both $F$. occidentalis and $T$. tabaci, thus showing its general applicability as a source for breeding thripsresistant Capsicum varieties.
\end{abstract}




\section{Introduction}

Thrips are a major pest in vegetable, fruit and ornamental crops worldwide (Kirk and Terry, 2003). Thrips is the common name for insects of the order of Thysanoptera, that includes over 5500 described species. Thrips damage the plant directly by feeding on the flowers, fruits and leaves, leading to silvering damage, deformation, reduced growth, and altered carbon allocation, resulting in reduced marketable yield (Chisholm and Lewis, 1984; Welter et al., 1990; Shipp et al., 1998a). However, the indirect way in which thrips affect their hosts, which is through the transmission of mainly tospoviruses, causes much larger economic losses in many crop plants, making thrips an important threat to the agricultural and horticultural sector (Mumford et al., 1996; Riley et al., 2011).

Only a few thrips species are currently reported to transmit tospoviruses, of which Western flower thrips (Frankliniella occidentalis Pergande) is the most important due to its worldwide occurrence (Ullman et al., 2002; Kirk and Terry, 2003; Riley et al., 2011). Western flower thrips has become the most important pest species in European greenhouses as well as in fields and orchards in the Mediterranean climate since its accidental introduction in 1983 (Van Lenteren and Loomans, 1999).

Before introduction of $F$. occidentalis from western North America, Thrips tabaci was the most prevalent thrips species in Europe (Van Lenteren and Loomans, 1999). Nowadays, T. tabaci is a major pest in onion, leek, cabbage, tobacco, garlic and cotton, but it also infests greenhouses crops such as tomato, pepper and cucumber, and ornamental crops. Thrips tabaci is also an important vector for plant viruses, among which iris yellow spot virus (IYSV) is the most important threat to onion (Kritzman et al., 2001; Gent et al., 2006). Thrips tabaci has also been reported to transmit TSWV, although studies have shown that only malefemale populations can transmit some isolates of TSWV and do so at a low rate (Wijkamp et al., 1995).

Therefore, it is important to explore the use of suitable sources for breeding broad spectrum thrips-resistant varieties. Host plant resistance to thrips has been previously observed and further characterized in Capsicum (Maharijaya et al., 2011; Maharijaya et al., 2012; Van Haperen et al., 2019; Visschers et al.; Visschers et al., 2019a). Maharijaya et al. (2011) identified CGN16975 as a resistant accession, as lower damage scores for both Thrips parvispinus (Karny) and $F$. occidentalis were observed. A significant inhibition of larval development of $F$. occidentalis first instar larvae was observed on leaves of this accession (Maharijaya et al., 2012; Van Haperen et al., 2019). The resistance to $F$. occidentalis was mapped to a single QTL on chromosome 6 that explained about 
$50 \%$ of the variation (Maharijaya et al., 2015). However, it is not known whether the QTL also has an effect on thrips when crossed into other susceptible genetic backgrounds. If the QTL is functional in other backgrounds, the QTL can be used to breed for thrips-resistant varieties.

The goal of this study is to further explore the potential of this QTL in different breeding programs for thrips-resistant varieties. We aim to confirm the effect of the QTL on F. occidentalis larval development on chromosome 6 in four Capsicum annuum backgrounds, obtained from two different breeding companies. In addition, we study the effect of the QTL on T. tabaci larval development in the four genetic backgrounds to determine whether this source of resistance is effective to multiple thrips species.

\section{Material and Methods}

\section{Overview of experiments and plant material}

In this study, larval development of Frankliniella occidentalis and Thrips tabaci were studied in four different genetic backgrounds (background 1 and 2 in experiment $1 A-B$, background 3 and 4 in experiment $2 A-B$ ). Plants with the resistance allele in homozygous state (" $R$ ") and plants with the susceptibility allele in homozygous state ("S") for the QTL region on chromosome 6 were selected for phenotyping with $F$. occidentalis (experiment $1 \mathrm{~A}$ and $2 \mathrm{~A}$ ) and $T$. tabaci (experiment $1 \mathrm{~B}$ and $2 \mathrm{~B}$ ).

An overview of plant material per experiment can be found in Table 1 . BASF Vegetable Seeds Netherlands produced the two BC3S1 introgression lines that resulted from crosses between the resistance donor Capsicum annuum CGN16975 and two susceptible $C$. annuum breeding lines that were used as backcross parents for each population (BP1 with background 1, and BP 2 with background 2, Table 2). Bejo Zaden BV produced two BC2S1 lines that resulted from a cross between resistant F3 plant from the mapping population of Maharijaya et al (2015) with the QTL region on chromosome 6 in homozygous state and two susceptible C. annuum backgrounds (background 3 and 4, Table 2). Markers flanking the QTL on chromosome 6 were used to select for the presence of the resistance allele in the next generations (Table 1). Background selection was applied for backgrounds 3 and 4 using SNP markers evenly distributed over all chromosomes. BC3 plants (background 1 and 2) and BC2 plants (background 3 and 4) that were heterozygous for the QTL region on chromosome 6 were selfed to obtain plants with either the resistance or the susceptibility allele in homozygous state for the QTL region on chromosome 6 in the different backgrounds. 
We grew plants from the BC2S1 seeds with background 3 and 4 , and simultaneously, we grew plants from a first generation inbred line (CGN16975A) of a CGN16975 plant from a CGN16975 seed batch obtained from the Centre for Genetic Resources, The Netherlands, and from the same seed batch (resistant F3 line) that was used as a resistance donor for the BC2S1 as resistant references. A second generation inbred line from susceptible accession CGN17219 (CGN17219A) was used as a susceptible reference. All seeds were sown end of March 2019 in potting compost in a greenhouse of Unifarm, Wageningen University and Research, Wageningen, the Netherlands. The plants were grown at $25^{\circ} \mathrm{C}$, with a photoperiod of L16:D8, and $70 \%$ relative humidity. Orius laevigatus (Fieber) was obtained from Entocare C.V. (Wageningen, the Netherlands) and used to control thrips during culturing. Plants were watered three times a week. Two times per week, nutrients were added. For the BC3S1 background 1 and 2, seeds were sown end of June 2018 together with CGN16975A as resistant reference and backcross parents 1 and 2 (BP1 and BP2) as susceptible references. These plants were grown under the same conditions as the BC2S1 plants.

Table 1: Overview of the experiments and plant material. Larval development of $F$. occidentalis was studied in experiments indicated with A. T. tabaci larval development was studied in experiments indicated with $B$. " $R$ " indicates plants that have the resistance allele in homozygous state for the QTL region on chromosome 6, whereas " $\mathrm{S}$ " indicates plants that the susceptibility allele in homozygous state for QTL region.

\begin{tabular}{|l|l|l|}
\hline Plant line & QTL chromosome 6 & Experiment \\
\hline CGN16975A & R & $1 A-B, 2 A-B$ \\
\hline Backcross parent 1 (BP1) & S & $1 A-B$ \\
\hline Backcross parent 2 (BP2) & S & $1 A-B$ \\
\hline Resistant F3 & R & $2 A-B$ \\
\hline CGN17219A & S & $2 A-B$ \\
\hline BC3S1 background 1 & R or S & $1 A-B$ \\
\hline BC3S1 background 2 & R or $S^{1}$ & $1 A-B$ \\
\hline BC2S1 background 3 & R or S & $2 A-B$ \\
\hline BC2S1 background 4 & R or $S^{1}$ & $2 A-B$ \\
\hline
\end{tabular}

1 " $\mathrm{R}$ or $\mathrm{S}$ " indicates that plants that either have the " $\mathrm{R}$ " or " $\mathrm{S}$ " allele in homozygous state were selected.

Table 2: Background information on the four backcross parents (experiment 1A-B and 2A-B). Information on BP1 and BP2 is kindly provided by BASF Vegetable Seeds Netherlands. Information on BP3 and BP4 is kindly provided by Bejo Zaden BV

\begin{tabular}{|l|l|l|l|l|l|}
\hline $\begin{array}{l}\text { Backcross } \\
\text { parent }\end{array}$ & Type & $\begin{array}{l}\text { Pungent/not } \\
\text { pungent }\end{array}$ & Shape & $\begin{array}{l}\text { Length } \\
\text { Width }\end{array}$ & Other remarks \\
\hline BP1 & Lamuyo & Not pungent & Lobed & $16 \times 5.5 \mathrm{~cm}$ & Red when fully ripened \\
\hline BP2 & Demre & Not pungent & Pointed & $20 \times 2.5 \mathrm{~cm}$ & Red when fully ripened \\
\hline BP3 & $\begin{array}{l}\text { Korean } \\
\text { hot }\end{array}$ & Pungent & Pointed & $\begin{array}{l}10-13 \times \\
2-2.5 \mathrm{~cm}\end{array}$ & $\begin{array}{l}\text { Red when ripened, dried and } \\
\text { processed into powder }\end{array}$ \\
\hline BP4 & Shandong & $\begin{array}{l}\text { Slightly } \\
\text { pungent }\end{array}$ & Pointed & $25-30 \times 5 \mathrm{~cm}$ & Harvested when green \\
\hline
\end{tabular}




\section{DNA extraction and genotyping}

Leaves from the BC3S1 plantlets were sampled at a plant age of 2.5 weeks and sent to BASF Vegetable Seeds (Nunhems) for DNA extraction and genotyping. Leaves from the BC2S1 plantlets were sampled at a plant age of 3 weeks and sent to Bejo Zaden BV for DNA extraction and genotyping. KASP-markers were designed based on SNPs flanking the 2-lod interval of the QTL region as previously defined by Maharijaya et al. (2015) (Table 3) and used for plant selection. Plants with the resistance or susceptibility allele in the QTL-region on chromosome 6 in homozygous state were selected for phenotyping at a plant age of 10 weeks (BC2S1) or 12 weeks (BC3S1).

Table 3: Markers used for genotyping the backcross lines. Shown are the flanking sequences of the QTL-regions on chromosome 6 showing the SNPs between brackets.

\begin{tabular}{|l|l|}
\hline $\begin{array}{l}\text { Marker } \\
\text { ID }\end{array}$ & Flanking region SNP \\
\hline M2 & $\begin{array}{l}\text { GTGAGGAAAAGAAAAGTTTGTCGTATGAGATTACCTTTACTAGTAAGAGCAGGGGT } \\
\text { GGAGTGGAGATGGT[A/G]GAGGGGGTTCAATCTGCATTTGGATCTATTGAGTGGA } \\
\text { GTGATGGGGTTCACAATGTGAGAAGTCCAATTG }\end{array}$ \\
\hline M15 & $\begin{array}{l}\text { CAAGAATTGGATGAATTCTACAGAGACTTGCGCAACAATTGGAATATCG[C/T]GAA } \\
\text { CAAAATGAGGTCCCGGAGAGAAACAACATCGATGAAGAATCATTAAA }\end{array}$ \\
\hline
\end{tabular}

\section{Thrips rearing and synchronization}

A population of Frankliniella occidentalis (Pergande) and a population of Thrips tabaci (Lindeman) were used to phenotype the plants for thrips resistance. The population of $F$. occidentalis originated from Greenhouse Horticulture of Wageningen University and Research, Bleijswijk, the Netherlands and was reared on Phaseolus vulgaris beans. The population of $T$. tabaci originated from Allium ampeloprasum var. porrum plants grown in a tunnel near Unifarm, Wageningen University and Research, Wageningen, the Netherlands and reared on leaf pieces of Allium ampeloprasum var. porrum. Each population was maintained in glass jars covered with a thrips proof gauze at $25^{\circ} \mathrm{C}, \mathrm{L} 16: \mathrm{D} 8$, and $70 \%$ relative humidity.

Synchronized first instar larvae (L1) of both thrips species were obtained by allowing female adults to lay eggs for 24 hours on snack cucumbers, after which the adults were removed and the cucumbers were transferred to a new glass jar and kept at a growth cabinet at $25^{\circ} \mathrm{C}$. After four days for F. occidentalis and five days for $T$. tabaci, the new synchronized first instar larvae emerged.

\section{Detached leaf assay}

Larval development from first into second instar larvae was used as a parameter for thrips resistance. The first four fully opened leaves of each plant were 
collected. Each leaf was placed with the petiole in $1.5 \%$ water agar in a Petri Dish (BD Falcon, tight-fit, $50 \times 9 \mathrm{~mm}$ ). Five first instar larvae were added to each leaf. At 3, 5 and 7 days after infestation, the developmental stage of the larvae was determined. The fraction of first instar larvae that did not develop into the next developmental stage was used as a resistance parameter. This development occurs in 1-2 days ( $F$. occidentalis) and 1-3 days ( $T$. tabaci) under optimal conditions (Lublinkhof and Foster, 1977).

\section{Statistics}

The fraction $L 1$ was transformed as $y=\operatorname{arcsine}(\sqrt{ } x)$. Fractions $L 1$ on BC2S1 plants were analysed separately from fractions L1 on BC3S1 plants, as these experiments were conducted at different time points. The transformed fractions L1 on BC3S1 (experiment 1) and BC2S1 plants (experiment 2) was used in two Three-Way analysis of variance (ANOVAs) to see whether there was an effect of the QTL genotype carrying either the resistance " $R$ " or susceptibility " $S$ " allele for the QTL region on chromosome 6, an effect of background (background 1 and 2, and background 3 and 4 respectively), and an effect of thrips species on larval development. When no significant three-way interaction was observed, the Threeway ANOVAs was repeated excluding the three-way interactions to confirm the significant two-way interactions. Similarly, two-way interactions that were not significant $(P>0.05)$ were excluded. A post hoc Fisher's Protected LSD was used to determine which groups show significant differences in larval development.

\section{Results}

Two Three-Way ANOVAs with QTL genotype ("R" or "S" allele), thrips species ( $F$. occidentalis or T. tabaci) and genetic background (background 1 and 2, and background 3 and 4 respectively) as factors followed by a Fisher's Protected LSD were used to determine the effects of each factor on larval development, possible interactions between these factors, and whether larval development was significantly different between which different groups (Figure 1 and 2; Supplementary data Table S1 and Table S2). No significant three-way interactions were observed in both Three-Way ANOVAs, hence, the Three-Way ANOVAs were repeated excluding the three-way interactions (Table S1 and S2). Also, the twoway interactions that did not have a significant effect were excluded (i.e. the interactions between QTL genotype and background, and between QTL genotype and thrips species in background 1 and 2 (Table S1), and the interactions between thrips species and background, and between thrips species and QTL genotype in background 3 and 4 (Table S2)). We observed a significantly higher fraction of L1 on leaves of plants that have the resistance (" $R$ ") allele in homozygous state for the QTL region on chromosome 6, than on leaves of plants that have the susceptibility ("S") allele for the QTL region, both in the BC3S1 lines (Background 
1 and 2, Figure 1; experiment 1 ) and in the BC2S1 lines (Background 3 and 4, Figure 2; experiment 2). A high fraction of L1 indicates resistance as only few L1s developed into the next larval stage. A significant difference between group " $R$ " and group " $\mathrm{S}$ " was observed for both thrips species $(\mathrm{P}<0.001$ in all cases). In addition, we observed a significant difference between the two thrips species on the different groups of plants. Frankliniella occidentalis shows a significantly lower fractions of L1 compared to T. tabaci. In background 1 and 2 (experiment 1 ) this difference is observed in all QTL genotypes, whereas in background 3 and 4 (experiment 2), this difference is only observed in the plants with the " $\mathrm{S}$ " allele for the QTL region on chromosome 6 and the susceptible reference. We also observed a significant difference in $F$. occidentalis larval development when comparing group " $\mathrm{R}$ " and group " $\mathrm{S}$ " with genetic background 1 to group " $\mathrm{R}$ " and group " $S$ " with genetic background 2 respectively, whereas these groups with different backgrounds do not show a significant difference in $T$. tabaci larval development. This observation resulted in a significant interaction between background and thrips species for background 1 and $2(P=0.004)$. This interaction was not observed in background 3 and $4(P=0.294)$. In genetic background 3 and 4 , we observe a significantly higher fraction L1 for each thrips species in group " $R$ " with background 4, compared to group " $R$ " with background 3, whereas the fractions $L 1$ in group " $S$ " did not significantly differ between backgrounds for each thrips species respectively. This observation resulted in a significant interaction between QTL genotype (group " $R$ " and group " $S$ ") and background $(P<0.001)$. No significant interaction was observed in background 1 and $2(P=0.611)$. 
Larval development in different genetic backgrounds (BC3S1)

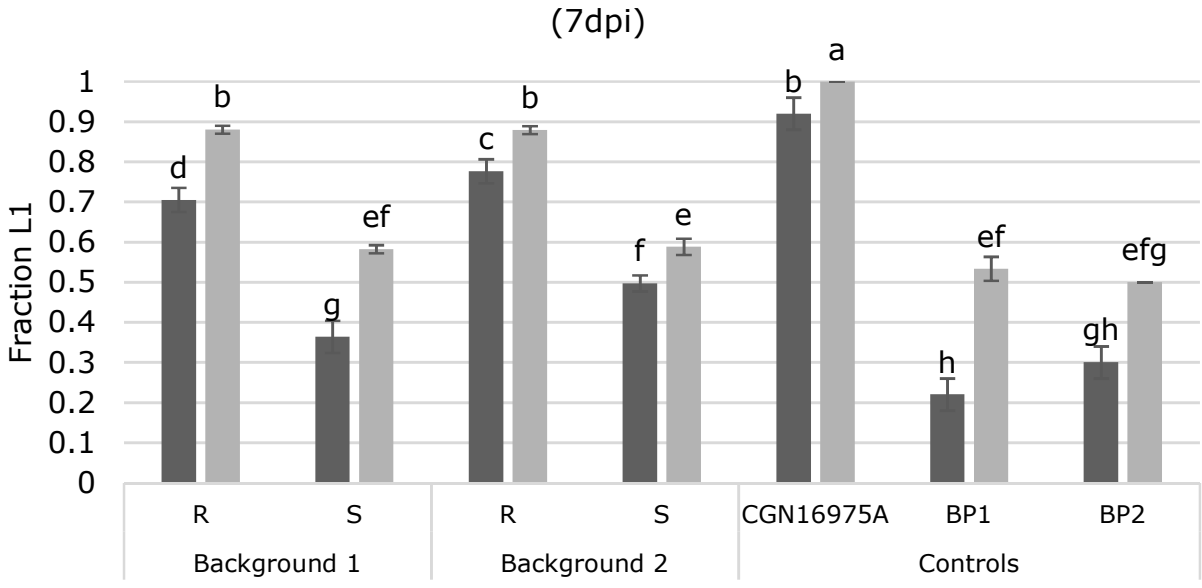

F. occidentalis $\square$ T. tabaci

Figure 1: Larval development on BC3S1 plants with either the resistance allele (R) or the susceptibility allele (S) in homozygous state for the QTL region on chromosome 6 in two different genetic backgrounds. CGN16975A was included as a resistant reference. The two susceptible backcross parents (BP1 and BP2) were included as susceptible references. Bars sharing the same letter do not show a significant difference (Fisher's Protected LSD, P>0.05).

Larval development in different genetic backgrounds (BC2S1) (7dpi)

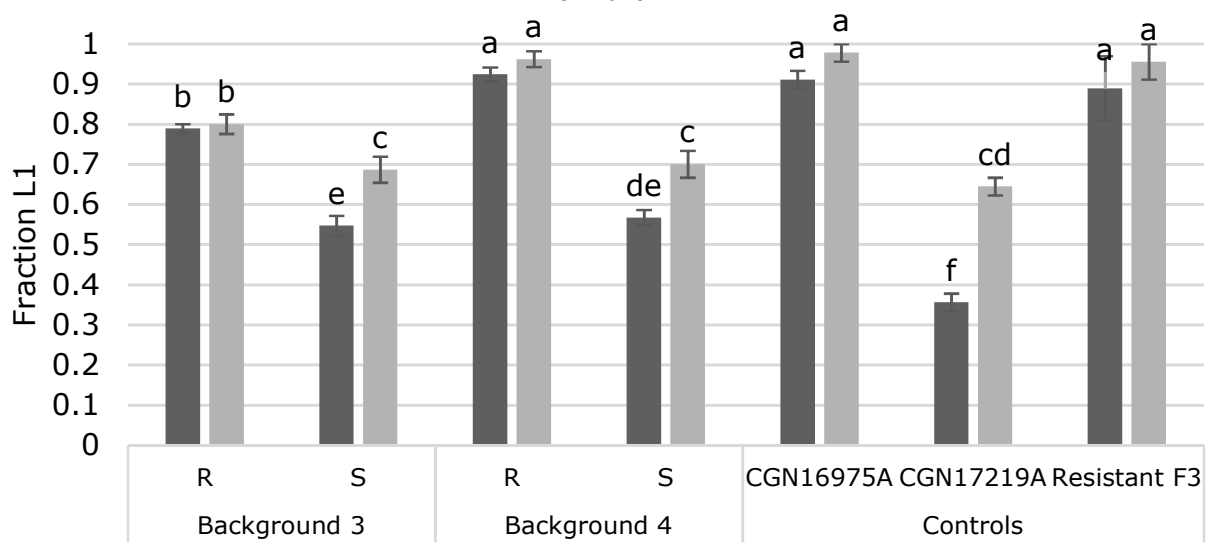

$\square$ F. occidentalis $\square$ T. tabaci

Figure 2: Larval development on BC2S1 plants with either the resistance allele (R) or the susceptibility allele (S) in homozygous state for the QTL region on chromosome 6 in two different genetic backgrounds. CGN16975A and plants from the F3 line that was used as a resistance donor (Resistant F3), were used as resistant controls. CGN17219A was included as a susceptible reference. Bars sharing the same letter do not differ significantly from each other (Fisher's Protected LSD, P>0.05) 


\section{Discussion}

In this study we showed the effect of the QTL on chromosome 6 in different Capsicum annuum backgrounds, using two thrips species. The physical distance between the current flanking markers of this QTL is $0.4 \mathrm{Mbp}$, and fifteen candidate genes are predicted in this region (Chapter 3). Pepper plants carrying the CGN16975 resistance allele in homozygous state for the QTL region on chromosome 6 showed a significantly higher level of thrips resistance to both $F$. occidentalis and T. tabaci in all four backgrounds tested, than plants that carry the susceptibility allele in homozygous state for this QTL region. This indicates that either one of the candidate genes affects larval development in both thrips species, or that two closely linked genes each affect one of the thrips species. We also observed significant differences in thrips resistance between the different backgrounds: $F$. occidentalis resistance in background 2 was significantly higher compared to background 1 , and background 4 had significantly higher levels of resistance to both thrips species compared to background 3 . This indicates that additional factors in the genetic background outside of the QTL region also contribute to thrips resistance, either affecting F. occidentalis, T. tabaci, or both thrips species. Maharijaya et al. (2015) calculated a broad sense heritability of 0.93 to 0.96 for the $F$. occidentalis resistance parameters, which suggests that the phenotypic variation is hardly affected by environmental factors (within the setup used in these experiments). Therefore there must be genetic factors other than the QTL on chromosome 6 that also affect resistance. This is as expected, as the QTL on chromosome 6 explains only 45 to 50 percent of the variance in the F2 population of Maharijaya et al. (2015). The genetic factors determining the unexplained part of the variance were presumably too small to be detected in the original mapping population, as was also suggested by Maharijaya et al. (2015). Also in Arabidopsis thaliana (Méndez-Vigo et al., 2013) and rice (Liao et al., 2001; Cheng et al., 2011) a significant effect of genetic background on QTLs has been shown. Therefore it is important to validate the effect of QTLs of interest in plants that have different alleles for the QTL region, but also show high similarities in the background outside of the QTL region. In addition, it is important to validate the effect of the QTL in different backgrounds, as additional factors that affect thrips resistance might be present or lacking.

We show that the resistance mechanism underlying the QTL, inhibition of larval development, affects both $F$. occidentalis and $T$. tabaci in all backgrounds tested. A significantly higher level of inhibition of larval development was observed for both thrips species in plants that carry the resistance allele for the QTL region, compared to plants that carry the susceptibility allele. In addition, the resistance source CGN16975, from which the QTL is derived, showed a significant effect on thrips damage caused by Thrips parvispinus (Karny) (Maharijaya et al., 2011). 
However, as the mapping of the resistance QTL derived from CGN16975 was done by screening larval development and damage of $F$. occidentalis, we do not know whether our QTL also affects larval development of T. parvispinus. Nevertheless, our study of the QTL effect on two thrips species shows that this QTL is a valuable source for breeding varieties with a broad range of resistance to thrips. Similar findings of QTLs conferring resistance to natural thrips populations consisting of two thrips species, i.e. T. tabaci and Frankliniella schultzei (Trybom), was found in cowpea, although it was not determined whether each QTL contributed to resistance to both thrips species, or only to one of the tested thrips species (Muchero et al., 2010). Contrary to our observations, no correlation was found between resistance to $F$. occidentalis and $T$. tabaci in pepper (Visschers et al., 2019a). However, in that study overall thrips resistance was compared in different pepper accessions, while our study focused on the effect of a QTL on thrips resistance within and between different backgrounds. In a follow up study Visschers et al. (2019) also evaluated other thrips species, in which they found a correlation between resistance to $F$. occidentalis and Scirtothrips dorsalis (Hood), but not to Thrips palmi (Karny) in C. annuum.

In the experiments with lines containing background 1 and 2, as well as with lines containing background 3 and 4 , interactions between different main factors (i.e. genetic background and thrips species, and genetic background and QTL genotype respectively) were observed. These interactions can be explained when further analysing the main observations in the different experiment. When comparing lines with background 1 to lines with background 2, we observe significant differences in plants with the same QTL genotype (group "R" background 1 to group " $\mathrm{R}$ " with background 2, and group " $\mathrm{S}$ " with background 1 and group " $\mathrm{S}$ " with background 2 respectively) when screened with $F$. occidentalis, and not when screened with $T$. tabaci. This might indicate that additional genetic factors that affect $F$. occidentalis, but not $T$. tabaci performance are present, either factors in background 1 that increase $F$. occidentalis performance, or factors in background 2 that decrease $F$. occidentalis performance, and thus explain the significant interaction between these factors. In the experiment in which lines with background 3 and 4 were tested, we observed a significant interaction between QTL genotype (group " $\mathrm{R}$ " and group " $\mathrm{S}$ ") and background (background 3 and background 4). This interaction can be explained, because we observed a significantly higher resistance to both thrips species in background 4 compared to

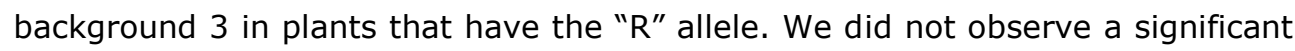
difference in larval development for both thrips species when plants have the " $S$ " allele. These results indicate the presence of a factor or factors in background 4 that only enhance thrips resistance when the " $R$ " allele for the QTL region on chromosome 6 is present. We did not observe a significant interaction between 
QTL genotype and background in the experiment with lines with background 1 and 2. These differences between the group " $R$ " and group " $S$ " with different backgrounds, screened with two thrips species, show that other genetic factors outside of the QTL region contribute to thrips resistance, and that these factors and their interactions should be taken into account in future research.

Altogether, this study shows the importance of studying the effect of a QTL in different genetic backgrounds, as our study indicates that different factors in the tested genetic backgrounds affect thrips resistance. Further elucidation of the resistance mechanism underlying the QTL might help in pinpointing these additional factors and their role in enhancing or impeding thrips resistance. Most importantly, we show that our QTL has a major effect on resistance to two thrips species in all four tested genetic backgrounds; thus we expect that this QTL will also be effective in other genetic backgrounds. Our findings show that this QTL can be used for breeding thrips-resistant Capsicum varieties.

\section{Acknowledgement}

We thank Betty Henken for her assistance with carrying out the detached leaf assay with the BC3S1 material. We thank Nunhems Netherlands B.V. and Bejo Zaden B.V. for providing the BC3S1 and BC2S1 seeds and for their help in genotyping the plants. This research was supported by a grant (TKI 1409-045) from the Ministry of Economic Affairs of the Netherlands and by breeding companies Bejo Zaden B.V. and Nunhems Netherlands B.V.

\section{Author contributions}

$\mathrm{PVH}, \mathrm{BV}, \mathrm{REV}$ and JVL conceived and designed the experiments. AFL and WS produced the BC3S1 and BC2S1 respectively. $\mathrm{PVH}$ carried out the detached leaf assays, data analysis and produced the first draft of the manuscript. BV, REV and JvL were involved in revising the manuscript. 


\section{Supplementary data}

Table S1: Three-way ANOVA summary table for the effect of QTL genotype (group " $R$ " and group " $S$ "), background ( 1 and 2 ) and thrips species (Frankliniella occidentalis and Thrips tabaci) and interactions between these factors on thrips larval development on BC3S1 plants. The three-way interaction $(P=0.883)$ and two-way interactions between QTL genotype and background ( $P=0.612)$, and QTL genotype and thrips species $(P=0.823)$ were excluded from this analysis as they were not significant $(P>0.05)$.

\begin{tabular}{|c|c|c|c|c|c|c|}
\hline \multicolumn{2}{|c|}{ Source of variation } & $\begin{array}{l}\text { Type III } \\
\text { Sum of } \\
\text { Squares }\end{array}$ & df & $\begin{array}{l}\text { Mean } \\
\text { Square }\end{array}$ & $F$ ratio & $\boldsymbol{P}$ \\
\hline \multicolumn{2}{|c|}{ Corrected model } & $5.931^{\mathrm{a}}$ & 4 & 1.483 & 80.398 & 0.000 \\
\hline \multicolumn{2}{|l|}{ Intercept } & 136.589 & 1 & 136.589 & 7406.620 & 0.000 \\
\hline \multirow[t]{3}{*}{$\begin{array}{l}\text { Main } \\
\text { effects }\end{array}$} & $\begin{array}{l}\text { QTL genotype } \\
\text { ("R" and "S") }\end{array}$ & 4.626 & 1 & 4.622 & 250.868 & 0.000 \\
\hline & $\begin{array}{l}\text { Background } \\
\text { (1 and } 2)\end{array}$ & 0.144 & 1 & 0.144 & 7.805 & 0.006 \\
\hline & $\begin{array}{l}\text { Thrips species } \\
\text { (Frankliniella } \\
\text { occidentalis } \\
\text { and Thrips } \\
\text { tabaci) }\end{array}$ & 1.014 & 1 & 1.014 & 54.989 & 0.000 \\
\hline $\begin{array}{l}\text { Interaction } \\
\text { effect }\end{array}$ & $\begin{array}{l}\text { Background } * \\
\text { Thrips species }\end{array}$ & 0.155 & 1 & 0.155 & 8.417 & 0.004 \\
\hline Error & & 2.600 & 141 & 0.018 & & \\
\hline Total & & 149.145 & 146 & & & \\
\hline Corrected T & & 8.531 & 145 & & & \\
\hline
\end{tabular}

a. R Squared $=0.695$ (Adjusted $\mathrm{R}$ Squared $=0.687$ ) 
Table S2: Three-way ANOVA summary for the effect of QTL genotype (group "R" and group " $S$ "), background ( 3 and 4) and thrips species (Frankliniella occidentalis and Thrips tabaci) and interactions between these factors on thrips larval development on BC2S1 plants. The three-way interaction $(P=0.337)$ and two-way interactions between thrips species and background $(P=0.392)$, and $Q T L$ genotype and thrips species $(P=0.248)$ were excluded from this analysis as they were not significant $(P>0.05)$.

\begin{tabular}{|c|c|c|c|c|c|c|}
\hline \multicolumn{2}{|c|}{ Source of variation } & $\begin{array}{l}\text { Type III } \\
\text { Sum of } \\
\text { Squares }\end{array}$ & d.f. & $\begin{array}{l}\text { Mean } \\
\text { Square }\end{array}$ & $F$ ratio & $\boldsymbol{P}$ \\
\hline \multicolumn{2}{|c|}{ Corrected Model } & $1.830^{\mathrm{a}}$ & 4 & 0.457 & 47.049 & 0.000 \\
\hline \multicolumn{2}{|l|}{ Intercept } & 48.900 & 1 & 48.900 & 5029.917 & 0.000 \\
\hline \multirow[t]{3}{*}{$\begin{array}{l}\text { Main } \\
\text { effects }\end{array}$} & $\begin{array}{l}\text { Background } \\
\text { (3 and } 4 \text { ) }\end{array}$ & 0.226 & 1 & 0.226 & 23.293 & 0.000 \\
\hline & $\begin{array}{l}\text { QTL genotype } \\
\text { ("R" and "S") }\end{array}$ & 1.123 & 1 & 1.123 & 115.478 & 0.000 \\
\hline & $\begin{array}{l}\text { Thrips species } \\
\text { (Frankliniella } \\
\text { occidentalis } \\
\text { and Thrips } \\
\text { tabaci) }\end{array}$ & 0.119 & 1 & 0.119 & 12.270 & 0.001 \\
\hline $\begin{array}{l}\text { Interaction } \\
\text { effect }\end{array}$ & $\begin{array}{l}\text { Background * } \\
\text { QTL genotype }\end{array}$ & 0.177 & 1 & 0.177 & 18.245 & 0.000 \\
\hline Error & & 0.379 & 39 & 0.010 & & \\
\hline Total & & 56.706 & 44 & & & \\
\hline Corrected T & & 2.209 & 43 & & & \\
\hline
\end{tabular}

a. $\mathrm{R}$ Squared $=0.828$ (Adjusted $\mathrm{R}$ Squared $=0.811$ ) 



\section{CHAPTER 6}

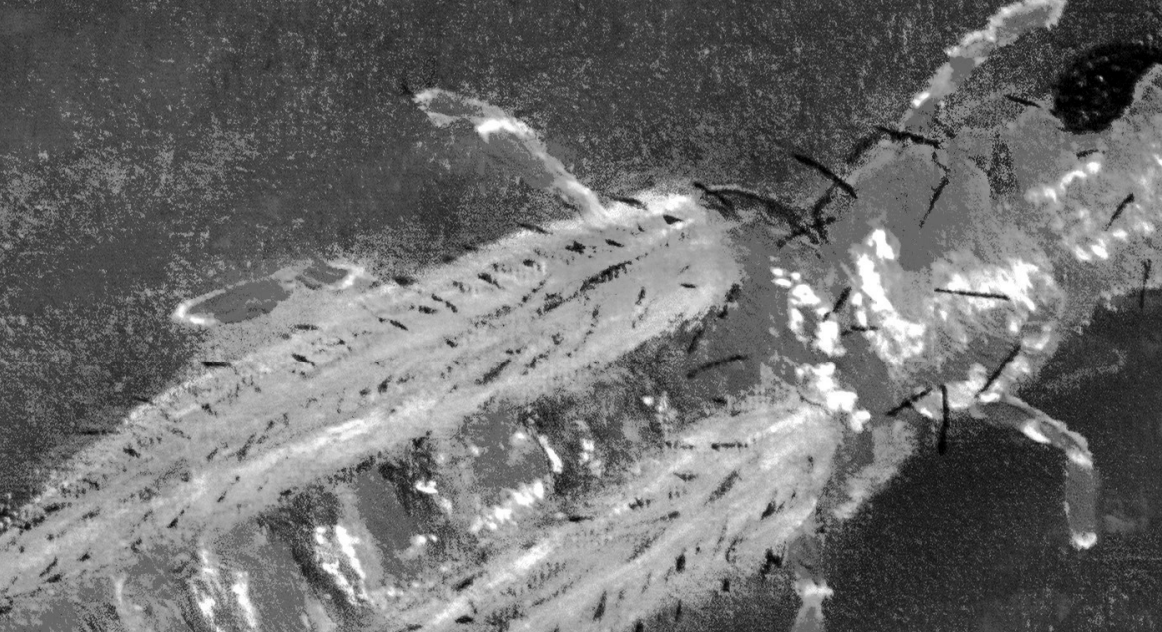

it
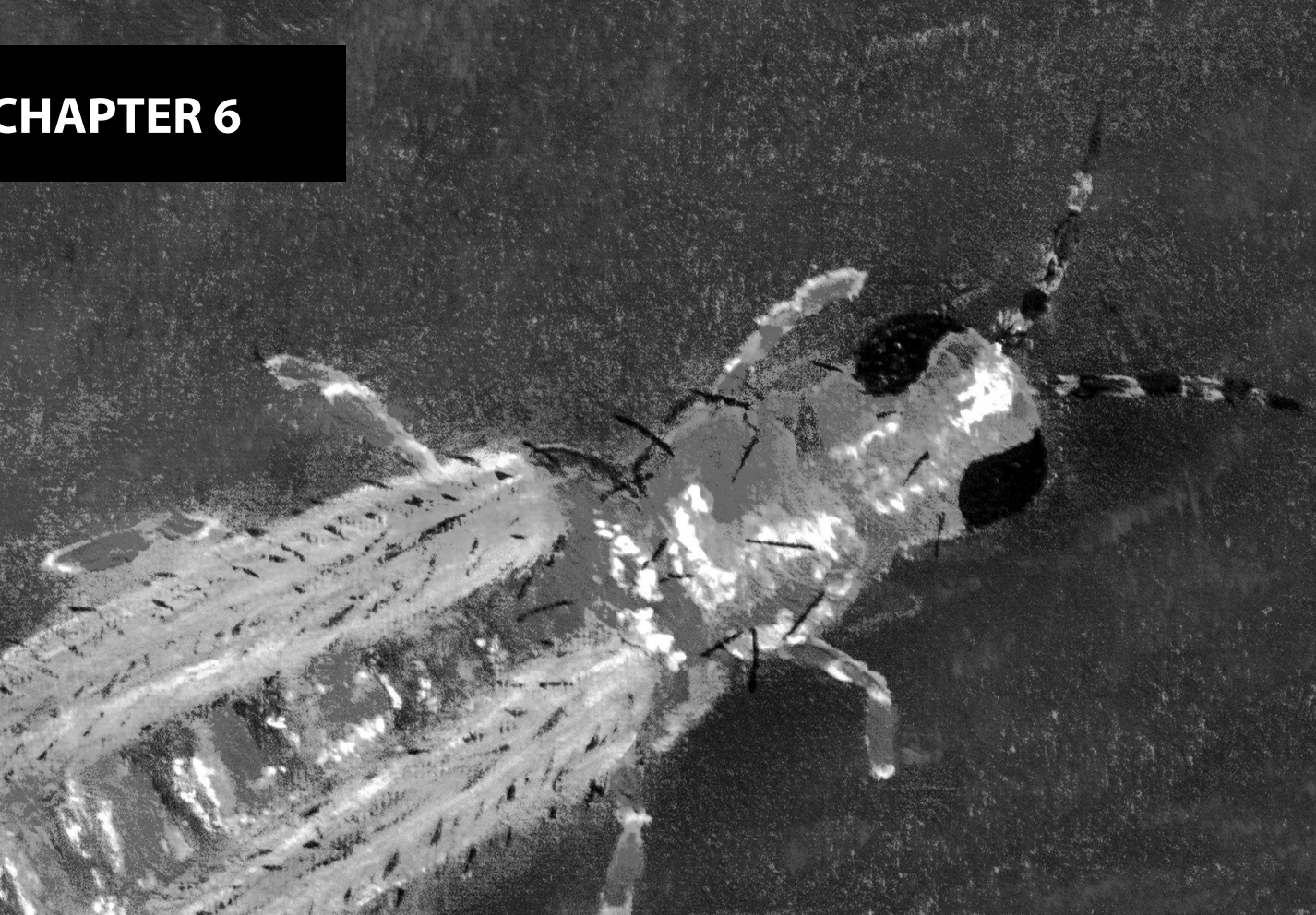


\section{The contribution of two QTLs to thrips resistance in Capsicum}

Paulinevan Haperen, Roeland E. Voorrips, Joop J.A. van Loon, Ben Vosman 


\begin{abstract}
Western flower thrips (Frankliniella occidentalis) is a major pest in pepper cultivation worldwide, causing large economic losses by feeding on leaves, fruits and flowers, and by transmitting tospoviruses. Therefore, host plant resistance against thrips is a desired trait and a target in breeding. Two independent mapping studies that used the same Capsicum annuum accession as a resistance source have identified one quantitative trait locus (QTL) each. One study identified a QTL on chromosome 5 , the other on chromosome 6 . This study aims to determine the contribution of each QTL, or the combination of QTLs, to thrips resistance in a nochoice setting. We did not detect any effect of the QTL on chromosome 5 on thrips resistance, neither on larval development, nor on silvering damage. In contrast to the QTL on chromosome 5, the contribution of the QTL on chromosome 6 was shown in both larval development and silvering damage assays, thus this QTL shows potential in breeding for thrips resistance. Possible reasons for the contrasting results of QTL mapping in the different studies are discussed.
\end{abstract}




\section{Introduction}

Western flower thrips (Frankliniella occidentalis Pergande) is an economically important pest on pepper worldwide (Shipp et al., 1998a). Thrips are very small insects that cause damage to pepper plants both directly and indirectly. The direct damage is mainly caused by thrips feeding on leaves, flowers and fruits, resulting in silvering damage, deformation, reduced plant growth and altered carbon allocation, thus reduced yield (Chisholm and Lewis, 1984; Welter et al., 1990; Shipp et al., 1998a). Indirect damage is caused through the role of thrips in vectoring plant viruses such as tospoviruses (German et al., 1992). Therefore, it is important to prevent thrips outbreaks in crop cultivation. However, it is difficult to control thrips due to their small size, cryptic behaviour, short life cycle and high reproduction capacity (reviewed by Cloyd (2009)). The use of biological control and insecticides can only reduce thrips population growth, but not completely eliminate thrips (reviewed by Mouden et al. (2017)). In addition, thrips can develop resistance to insecticides (reviewed by Jensen (2000)).

Host plant resistance is a desired trait for pepper growers. Resistance may affect the interaction between plant and insect in different ways. Depending on the resistance mechanism, these can be divided in antibiosis, antixenosis and tolerance. Antibiotic mechanisms affect the life history parameters of thrips, for instance by delaying larval development or by affecting their survival (Smith, 2005). Antixenotic mechanisms affect the behaviour of thrips, for instance by producing odours or chemicals that deter thrips from feeding (Smith, 1989). Tolerance is the ability of a plant to limit damage in the presence of thrips, without affecting their behaviour or life history parameters. Tolerance does not prevent thrips from feeding, thus tospoviruses vectored by thrips can still be transmitted. We are mainly interested in antibiotic resistance mechanisms, as thrips do not have a choice to feed on different crops or varieties in greenhouse cultivation.

Previous studies have shown host plant resistance to thrips in Capsicum accessions (Fery and Schalk, 1991; Maris et al., 2003; Maharijaya et al., 2011). One of the Capsicum accessions most resistant to $F$. occidentalis is CGN16975 (Maharijaya et al., 2011). Further characterization of this resistance source showed that larval development is inhibited on young leaves, and that the level of resistance is plant and leaf age dependent (Maharijaya et al., 2012; Van Haperen et al., 2019). Quantitative trait loci (QTL) for resistance to thrips in CGN16975 were mapped using F2 populations in two independent studies. Linders et al. (2015a) chose an unknown Capsicum annuum accession from the Westland area, The Netherlands, as susceptible parent. A single resistance QTL was mapped on chromosome 5 in a choice assay with plant damage as resistance parameter. Maharijaya et al. (2015) used a Capsicum chinense (CGN17219) as a susceptible 
parent and mapped a single thrips resistance QTL on chromosome 6 in a no-choice assay. Larval survival, pupal survival and larval damage were used as resistance parameters. Maharijaya et al. (2015) included four markers described in Linders et al. (2015) near their resistance QTL on chromosome 5 QTL, but did not detect an effect of that QTL on these parameters.

As both mapping studies used CGN16975 as resistance donor but identified different QTLs on different chromosomes, questions were raised about the cause of this difference and about the contribution of the two QTLs to thrips resistance. Therefore, the near isogenic line (NIL) described in the patent application by Linders et al. (2015) was requested and characterized in this study. The aim of this study is to determine the contribution of each QTL or the combination of QTLS to thrips resistance in no-choice assays. We chose to use no-choice assays as under commonly applied greenhouse cultivation thrips will have no choice among varieties.

\section{Material and Methods}

\section{Overview of experiments and plant material}

Plants from the F2 mapping population of Maharijaya et al. (2015) were selfed to obtain seeds of different lines for evaluation. Seeds of the resistant accession of the mapping population (CGN16975) were obtained from the Centre for Genetic Resources, The Netherlands). One CGN16975 plant was selfed, and the inbred line resulting from the selfing was used as a resistant reference (CGN16975A). An inbred line from the original susceptible parent of the mapping population (CGN17219) was used as a susceptible reference (CGN17219A). Seeds from the near isogenic line (NIL), NCIMB41428, that was developed and deposited by Linders et al. (2015) were obtained from NCIMB Ltd, Aberdeen, UK. According to Linders et al. (2015), plants from this NIL contain the CGN16975 allele for the thrips resistance QTL on chromosome 5 in homozygous state in a susceptible $C$. annuum background.

A description of the plant material studied in each experiment can be found in Table 1. In the study of larval development on NCIMB41428 and four F3 lines (experiment 1), we selected 4 F3 lines (Line 1 through 4) originating from F2 plants from the mapping population of Maharijaya et al. (2015). F3 lines 1 and 2 contain the resistance allele ("R") in homozygous state for the QTL region on chromosome 6 , and $\mathrm{F} 3$ lines 3 and 4 contain the susceptibility allele (" $\mathrm{S}$ ") for the same region. We compared larval development on leaves from these plants to larval development on leaves from plants of NCIMB41428, and to leaves from the resistant (CGN16975A) and susceptible (CGN17219A) reference. In the study of silvering damage on NCIMB41428 and four F3 lines (experiment 2), we compared 
the silvering damage in a no-choice assay on plants from F3 lines that originate from F2 plants from the mapping population of Maharijaya et al. (2015) with all four homozygous allele combinations of the QTLs on chromosome 5 and 6 (lines 5 through 8), with plants of NCIMB41428 and the resistant CGN16975A and susceptible CGN17219A references. In the study of larval development in an F2 population obtained from a cross between NCIMB41428 and CGN17219A (experiment 3), we screened F2 plants to see the effect of the QTLs that originate from NCIMB41428 on larval development, and we included NCIMB41428, CGN16975A and CGN17219A as references. To study the effect of the QTLs on chromosome 5 and 6 in different $C$. annuum backgrounds (experiment 4), we reanalysed the previously obtained data on larval development of BC3S1 plants with background 1 and 2 (Lamuyo type and Demre type respectively; Table 2 in Chapter 5), by dividing the plants into four groups with all possible QTL combinations for both QTL regions (i.e. "RR", "RS", "SR" and "SS"), thus studying the effect of the QTL on chromosome 5, on chromosome 6, or a combination of both QTLs. The BC3S1 plants result from crosses between CGN16975 and two fully homozygous susceptible Capsicum annuum lines, the backcross parents (BP1 and $\mathrm{BP} 2$ ). The $\mathrm{BC3}$ plants that were heterozygous for the QTL regions on chromosome 5 and 6 were selfed in order to obtain BC3S1 plants that have the resistance allele or the susceptibility allele in homozygous state for both QTL regions in all four combinations. Selfings from eight BC3S1 plants (BC3S2), that together have the four different QTL combinations in each background, were used in a whole plant damage assay in a no-choice setting (experiment 5).

For each line that was used in experiment 1 and 2, 5 plants were randomly selected. For experiment 3, we sowed 120 F2 seeds and selected plants with the resistance or susceptibility allele in homozygous state for both QTL regions. For experiment 4, we sowed 555 seeds for genetic background 1 and 658 seeds for genetic background 2 . We selected 10 plants for each QTL combination per background. All seeds were planted in potting soil in a greenhouse at Unifarm (Wageningen University and Research, Wageningen, The Netherlands), and all selected plants were grown at $25^{\circ} \mathrm{C}, 70 \% \mathrm{RH}$, and a photoperiod of L16:D8.

\section{Frankliniella occidentalis rearing and synchronization}

Western flower thrips (Frankliniella occidentalis Pergande) were reared on Phaseolus vulgaris pods in glass jars covered with a thrips proof gauze in a growth cabinet $\left(25^{\circ} \mathrm{C}, \mathrm{L} 16: \mathrm{D} 8,70 \% \mathrm{RH}\right)$. The population originated from the Greenhouse Horticulture department of Wageningen University and Research, Bleiswijk, The Netherlands. 
Synchronized first instar larvae (L1) were obtained by allowing female adults to lay eggs on snack cucumbers. After $24 \mathrm{~h}$, the thrips were removed and the snack cucumbers were placed in a new glass jar. After four days at $25^{\circ} \mathrm{C}$, new synchronized L1s which were used in the experiments emerged from the eggs.

Table 1: Overview of the plant material that was examined in different experiments to study the effect of the QTLs on chromosome 5 and 6 on larval development and silvering damage. F3 lines 1-8 were obtained by selfing 8 different F2 plants from the mapping population of Maharijaya et al. (2015). " $\mathrm{R}$ " and " $\mathrm{S}$ " indicate that the plants have the resistance or susceptibility allele in homozygous state for the specified QTL region, respectively. In experiment 1,3 and 4, larval development is studied in a detached leaf assay. In experiment 2 and 5 , silvering damage is scored in a whole plant damage assay.

\begin{tabular}{|c|c|c|c|}
\hline Plant Line & QTL chromosome 5 & QTL chromosome 6 & Experiment \\
\hline CGN16975A & $\mathrm{R}$ & $\mathrm{R}$ & $1-5$ \\
\hline CGN17219A & $\mathrm{S}$ & $\mathrm{S}$ & $1-3$ \\
\hline NCIMB41428 & $\mathrm{R}^{1}$ & Unknown ${ }^{2}$ & $1-3$ \\
\hline F3 Line 1 & Unknown & $\mathrm{R}$ & 1 \\
\hline F3 Line 2 & Unknown & $\mathrm{R}$ & 1 \\
\hline F3 Line 3 & Unknown & $\mathrm{S}$ & 1 \\
\hline F3 Line 4 & Unknown & $\mathrm{S}$ & 1 \\
\hline F3 Line 5 & $\mathrm{R}$ & $\mathrm{R}$ & 2 \\
\hline F3 Line 6 & $\mathrm{~S}$ & $\mathrm{R}$ & 2 \\
\hline F3 Line 7 & $\mathrm{R}$ & $\mathrm{S}$ & 2 \\
\hline F3 Line 8 & $\mathrm{~S}$ & $\mathrm{~S}$ & 2 \\
\hline $\begin{array}{l}\text { F2 population } \\
\text { NCIMB41428 } \\
\text { CGN17219A }\end{array}$ & $\mathrm{R}^{1}$ or $\mathrm{S}^{3}$ & Unknown ${ }^{2}$ or $\mathrm{S}^{3}$ & 3 \\
\hline BC3S1 background 1 & $\mathrm{R}$ or $\mathrm{S}^{3}$ & $\mathrm{R}$ or $\mathrm{S}^{3}$ & 4 \\
\hline BC3S1 background 2 & $\mathrm{R}$ or $\mathrm{S}^{3}$ & $\mathrm{R}$ or $\mathrm{S}^{3}$ & 4 \\
\hline BC3S2 background 1 & $\mathrm{R}$ or $\mathrm{S}^{3}$ & $\mathrm{R}$ or $\mathrm{S}^{3}$ & 5 \\
\hline BC3S2 background 2 & $\mathrm{R}$ or $\mathrm{S}^{3}$ & $\mathrm{R}$ or $\mathrm{S}^{3}$ & 5 \\
\hline $\begin{array}{l}\text { Backcross parent } 1 \\
\text { (BP1) }\end{array}$ & $\mathrm{S}$ & $\mathrm{S}$ & 4 \\
\hline $\begin{array}{l}\text { Backcross parent } 2 \\
(\mathrm{BP} 2)\end{array}$ & $\mathrm{S}$ & $\mathrm{S}$ & 4 \\
\hline \multicolumn{4}{|c|}{${ }^{1}$ according to Linders et al. (2015) } \\
\hline \multicolumn{4}{|c|}{${ }^{2}$ as Linders et al. (2015) did not select for either allele at the QTL position on chromoson } \\
\hline \multicolumn{4}{|c|}{6 it is not known which allele is present } \\
\hline \multicolumn{4}{|c|}{ 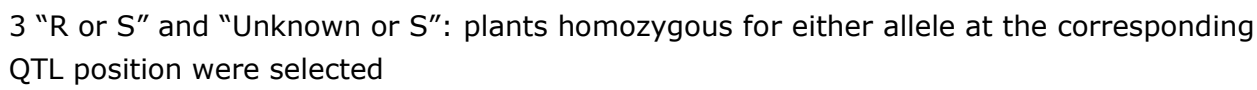 } \\
\hline
\end{tabular}




\section{DNA extraction and genotyping}

At a plant age of 3 weeks DNA was extracted from young leaves of each individual plant from the F2 population that resulted from the cross of CGN17219A as susceptible parent and NCIMB41428 as a resistant parent. The leaves were collected in cluster tubes ( 8 strips) with $2-\mathrm{mm}$ stainless steel beads each and stored at $-80^{\circ} \mathrm{C}$ overnight. Leaf samples were ground with the Tissuelyser II (Qiagen) for $70 \mathrm{~s}$ at $25 \mathrm{~Hz}$. The protocol to extract DNA from the leaf samples was adapted from Fulton et al. (1995). We added $500 \mu \mathrm{L}$ freshly prepared Microprep buffer to all samples, which were then shaken until homogenized. After at least 30 min of incubation in a $65^{\circ} \mathrm{C}$ water bath, samples were cooled down in ice water for 5 minutes. After cooling, $500 \mu \mathrm{L}$ chloroform was added and the samples were turned 40 times. Samples were centrifuged for $15 \mathrm{~min}$ at $3500 \mathrm{rpm}$ and the supernatant transferred to a new tube with 0.8 volume of isopropanol. The samples were turned 40 times and subsequently centrifuged for $15 \mathrm{~min}$ at 3500 rpm. The supernatant was discarded and the pellet was washed with $175 \mu \mathrm{L} 70 \%$ ethanol. After drying the pellets, they were resuspended in $100 \mu \mathrm{L} M Q$ and stored overnight at $4{ }^{\circ} \mathrm{C}$ before genotyping. Two flanking markers for each QTL region on chromosome 5 and 6 (Table 2) were used to determine the QTL genotypes for these regions. The markers were tested using the LightScanner ${ }^{\circledR}$ System (Idaho Technology Inc.) with the small amplicon approach (Liew et al., 2004). Based on the marker scores, plants with the desired homozygous genotype at both QTL positions were selected.

Table 2: Markers used to characterize the QTL regions on Chromosomes 5 and 6. LM2002 and LM2006 were designed to determine the genotype for the QTL region on chromosome 5, and based on variance in simple sequence repeats (SSRs) (Linders et al., 2015). M2 and M15 were used to determine the genotype for the QTL region on chromosome 6 , and based on two single nucleotide polymorphisms (SNPs; Table 2 in Chapter 3).

\begin{tabular}{|c|c|c|c|c|}
\hline $\begin{array}{l}\text { Marker } \\
\text { ID }\end{array}$ & $\begin{array}{l}\text { Marker } \\
\text { type }\end{array}$ & $\begin{array}{l}\text { QTL } \\
\text { chromosome }\end{array}$ & Forward primer $\left(5^{\prime}-3^{\prime}\right)$ & Reverse primer $\left(5^{\prime}-3^{\prime}\right)$ \\
\hline LM2002 & SSR & 5 & CCCGTTTACAAGCAAAGAG & GACCCCTGAAGAACCTCTC \\
\hline LM2006 & SSR & 5 & TCGGCCTGACTAGTATTGAC & CGGGTACCAGATGTAGGG \\
\hline M2 & SNP & 6 & ACTAGTAAGAGCAGGGGTG & $\begin{array}{l}\text { TCAATAGATCCAAATGCAGAT } \\
\text { TGAAC }\end{array}$ \\
\hline M15 & SNP & 6 & $\begin{array}{l}\text { CAAGAATTGGATAATGATTCT } \\
\text { TCAT } \\
\text { CGATGTTGTTGAATTCTACAG } \\
\text { AGA }\end{array}$ & $\begin{array}{l}\text { AATGATTCTTCATCGATGTTG } \\
T T\end{array}$ \\
\hline
\end{tabular}

Young leaves of the BC3S1 plants were sampled at a plant age of 2.5 weeks and sent to BASF Vegetable Seeds (Nunhems) for DNA extraction and genotyping. KASP-markers were designed based on the flanking sequences of the single nucleotide polymorphism (SNP) that flank the QTL region and used for plant 
selection. Plants with the resistance allele or susceptibility allele in homozygous state for the QTL regions on chromosome 5 and 6 were selected for phenotyping.

\section{Larval development in a detached leaf assay}

The youngest three fully opened leaves per plant were used to study larval development from first into second instar larvae (L1 into L2). The leaves were collected at a plant age of 12 weeks and placed with their petiole in $1.5 \%$ water agar in a Petri Dish (BD Falcon, tight-fit, $50 \times 9 \mathrm{~mm}$ ). Five synchronized L1 larvae (L1s) were added to each leaf. The developmental stage of the larvae was scored at 3,5 and 7 days post infestation (dpi). We used the fraction first instar larvae (fraction L1) that did not develop into second instar larvae (L2) at $7 \mathrm{dpi}$ as a resistance parameter.

\section{Whole plant damage assay}

In the first whole plant damage assay, experiment 2, F3 plants from different lines with different QTL combinations for the regions on chromosomes 5 and 6 were included. CGN16975A and CGN17219A were included as resistant and susceptible reference (previously screened in Chapter 2 ). In the second whole plant damage assay, experiment 4, plants with the different QTL combinations in two different susceptible Capsicum backgrounds were tested. Five plants were tested for each group of plants (i.e. plants from lines 5-8 in experiment 2, and BC3S2 plants with different QTL combinations in each background in experiment 4). Only two plants from NCIMB41428 could be included in the second whole plant damage assay due to poor germination. The same method and damage scale as described in Chapter 2 were used. Plants were randomized, covered with a thrips proof gauze, and placed in a water slot. Female adult thrips were collected using an aspirator and carefully anesthetized with carbon dioxide $\left(\mathrm{CO}_{2}\right)$. Twenty female adults were added to each plant at a plant age of 7 weeks. The infestation was repeated after one week to ensure high thrips pressure. For the first whole plant damage assay, the five most damaged leaves were scored for silvering damage three weeks after the first infestation. In the second assay the five most damaged leaves were scored five weeks after the first infestation. The damage was scored according to a scale from 1 (no damage) to 9 (heavy damage) as described in Chapter 2.

\section{Statistical analysis}

The fractions of L1 in the larval development tests were transformed as $y=\operatorname{arcsine}(\sqrt{ } x)$. A Shapiro-Wilk test for Normality was performed. If the data was not normally distributed, a Kruskal-Wallis One-Way Analysis of variance (ANOVA) was used to determine whether there was a significant difference in fractions of L1 between the different groups of plants. Dunn's post-hoc test was used to determine which groups significantly differed. If the data was normally distributed, 
a One-Way ANOVA was used, followed by a Fisher's Protected least-significantdifference (LSD) test, to determine which groups of plants showed a significant difference in fractions L1. A Three-Way ANOVA was used to determine the effect of the combinations of QTLs on chromosome 5 and 6 and the genetic background, the effect of each QTL separately on larval development on the BC3S1 plants and the potential interactions. When no significant interactions were observed, the Three-way ANOVA was repeated excluding possible interaction effects. A Fisher's Protected LSD test was used to determine which groups of plants show significant differences in fractions L1 within the same genetic background. A Mann-Whitney $\mathrm{U}$-test was used to determine the difference in silvering damage between plants with different Capsicum backgrounds.

A Kruskal-Wallis One-Way ANOVA was used to determine whether the silvering damage scores of leaves from plants with different QTL combinations in each background differed significantly. Dunn's post hoc test was used to determine significant differences in thrips resistance between plants with different genotype within each Capsicum background. A Mann-Whitney $U$ test was done to test whether plants with genetic background 1 showed a significant difference in silvering damage compared to plants from background 2 .

The Shapiro-Wilk test for Normality and One-Way ANOVA were carried out using Genstat (VSN International, 2015). The Three-Way ANOVA, Kruskal-Wallis OneWay ANOVA, Dunn's post hoc test, and Mann-Whitney $U$ test were carried out using IBM SPSS Statistics for Windows, Version 23.0 (IBM Corp., 2015).

\section{Results}

\section{Larval development on NCIMB41428 and four F3 lines}

To characterize the plant material that was filed by Linders et al. (2015a), we compared the larval development on NCIMB41428, the resistant reference CGN16975A, the susceptible reference CGN17219A and four F3 lines that either had the resistance (Line 1 and 2, "R") or the susceptibility (Line 3 and 4, "S") allele for the QTL region on chromosome 6 in homozygous state (experiment 1 ). The fraction L1 indicates the fraction of larvae that did not develop beyond the L1 stage, a high fraction signifying resistance. The fraction L1 on NCIMB41428 was significantly higher than the fraction L1 on the susceptible CGN17219, but significantly lower than the fraction L1 on the resistant CGN16975 (Figure 1). In addition, we observed a significant difference in larval development between Line 3 and 4, although both lines carry the " $\mathrm{S}$ " allele in homozygous state in the QTL region on chromosome 6 . Line 2 , which is one of the lines carrying the " $R$ " allele in homozygous state, had a significantly higher fraction L1 than both lines with 
the " $S$ " allele (Line 3 and 4). The difference in larval development between Line 1 , that has the " $\mathrm{R}$ " allele, and Line 4 , that has the " $\mathrm{S}$ " allele, was not significant.

\section{Silvering damage in NCIMB41428 and 4 F3 lines}

Silvering damage on the five most affected leaves was scored in plants with the resistance (" $R$ ") or susceptibility (" $\mathrm{S}$ ") allele in homozygous state for the QTL regions on chromosome 5 and 6 , and plants from NCIMB41428 (experiment 2). The resistant CGN16975A and susceptible CGN17219A were included for reference. Plants from NCIMB41428 received a significantly higher silvering damage score compared to the resistant CGN16975A, but also scored significantly lower on silvering damage compared to the susceptible CGN17219A (Figure 2). NCIMB41428 plants showed similar levels of silvering damage compared to plants from the F3 lines that were homozygous for the resistance allele of the QTL region on chromosome 6 . We observed significantly higher levels of silvering damage on plants that scored " $S$ " for markers in the QTL region on chromosome 6 (Line 7, Line 8 and CGN17219A) than plants that scored " $R$ " for this region (Line 5, Line 6 and CGN16975A). We did not observe significant differences between line 5 and 6 , and between line 7 and 8 , although these lines have different alleles for the QTL region on chromosome 5 (Line 5 and 7: "R" ; Line 6 and 8: "S").

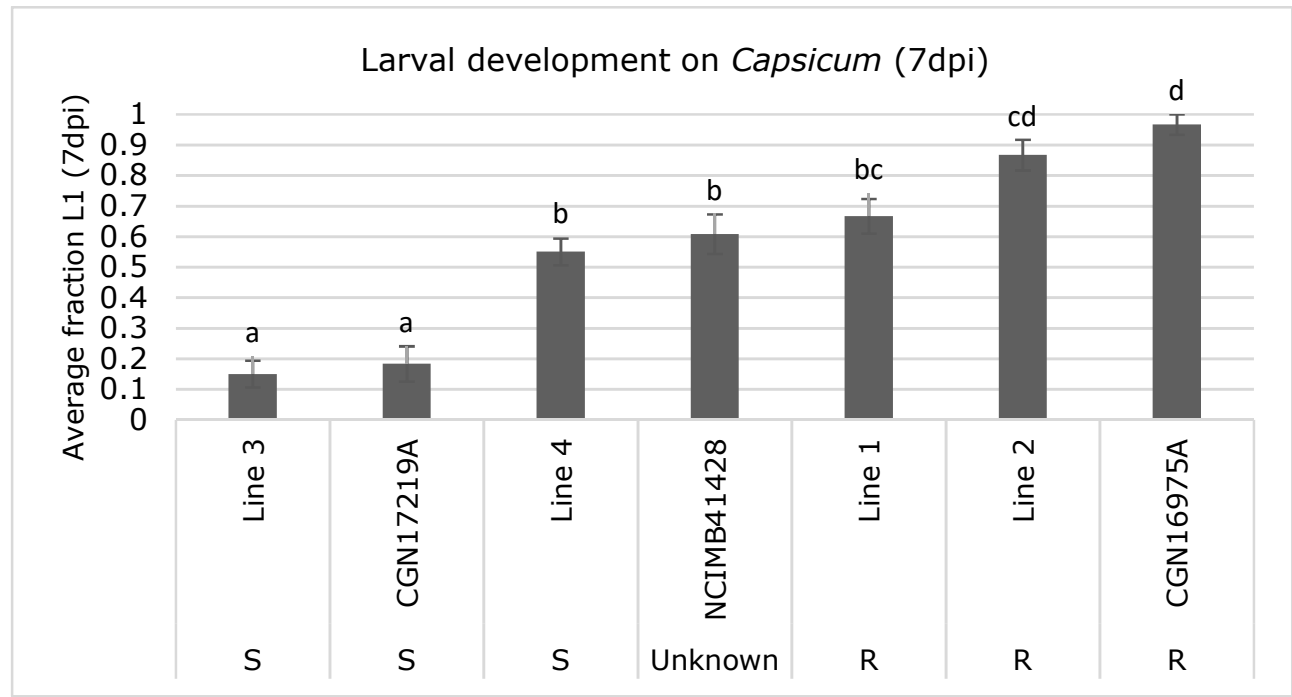

Figure 1: Larval development on NCIMB41428, CGN17219A and CGN16975A and four F3 lines that either have the resistance or susceptibility allele for the QTL region on chromosome 6 in homozygous state. Line 1-4 originate from two F2 plants from the mapping population as described by (Maharijaya et al., 2015). CGN16975A, line 1 and line 2 have the resistance ("R") allele for the QTL region on chromosome 6. CGN17219A, line 3 and line 4 have the susceptibility (" $\mathrm{S"}$ ) allele for the QTL region on chromosome 6. The resistant parent CGN16975A and the susceptible parent CGN17219A are included for reference. Bars sharing the same letter are not significantly different (Dunn's post hoc test, $\mathrm{P}>0.05)$. 


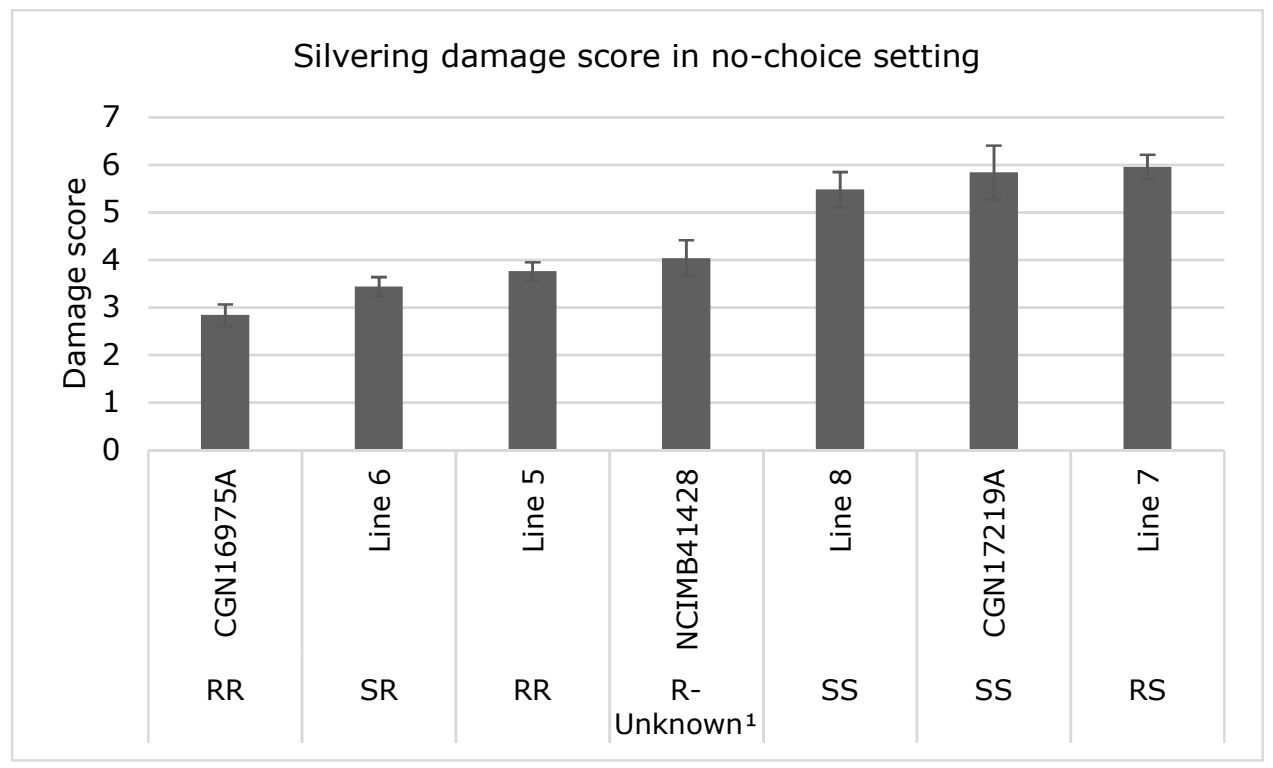

Figure 2: Silvering damage scores for four F3 lines with different QTL combinations for the QTL regions on chromosome 5 and 6, and for NCIMB41428, CGN16975A and CGN17219A. The 2-letter combination indicates whether the lines had the resistance ("R") or susceptibility ("S") allele in homozygous state for chromosome 5 (first letter) and 6 (second letter). Bars sharing the same letter are not significantly different (Dunn's post hoc test, $\mathrm{P}>0.05$ ). See Table 3 for scoring scale.

${ }^{1}$ As Linders et al (2015) did not select for the resistance or susceptibility allele for the QTL region on chromosome 6 , it is not known which allele is present in NCIMB41428

\section{Larval development on F2 plants obtained from a cross between NCIMB41428 and CGN17219A}

Larval development was studied on plants from the $\mathrm{F} 2$ population that resulted from a cross between a plant from NCIMB41428 and a plant from the susceptible CGN17219A (experiment 3). Plants from NCIMB41428, CGN17219A and CGN16975A were included as reference. Plants from NCIMB41428 had a significantly higher fraction L1 compared to plants from susceptible accession CGN17219, but a significantly lower fraction L1 compared to plants from resistant accession CGN16975 (Figure 3). The fraction L1 on plants from NCIMB41428 was similar to all groups of F2 plants with different QTL combinations. We also observed a significantly higher fraction L1 on plants with the resistance allele in homozygous state for both QTL regions on chromosome 5 and 6 ("RR") than on the susceptible CGN17219A, and on plants that have the susceptibility allele in homozygous state for the QTL region on chromosome 6 ("RS" and "SS"). 


\section{Larval development and silvering damage on plants with different QTL combinations in two C. annuum backgrounds}

The fraction L1 larvae that did not develop into instar L2 was determined on leaves from BC3S1 plants that either had the resistance allele or susceptibility allele in homozygous state for each of the QTL regions 5 and 6 (Figure 4; experiment 4). The effect of the QTL on chromosome 6 in these BC3S1 plants was previously evaluated (Chapter 5). In this experiment, we re-analysed the data to determine the effect of the chromosome 5 QTL on larval development of $F$. occidentalis. We divided the plants into four groups with all QTL combinations for both QTL regions on chromosome 5 and 6, i.e. "RR", "RS", "SR" and "SS". Plants with the resistance allele in homozygous state at the chromosome 6 QTL showed a significantly higher fraction of L1 than plants that have the susceptibility allele in homozygous state for this region. A Three-Way ANOVA to determine the effect of each QTL and the effect of the genetic background shows a significant effect of the QTL on chromosome $6(P<0.001)$ and a significant effect of the genetic background on larval development $(P=0.002)$. We did not observe a significant effect of the QTL on chromosome 5 on larval development $(P=0.304)$. All possible interactions between genetic background, effect of QTL on chromosome 5 or effect of QTL on chromosome 6 were not significant $(P>0.05)$.

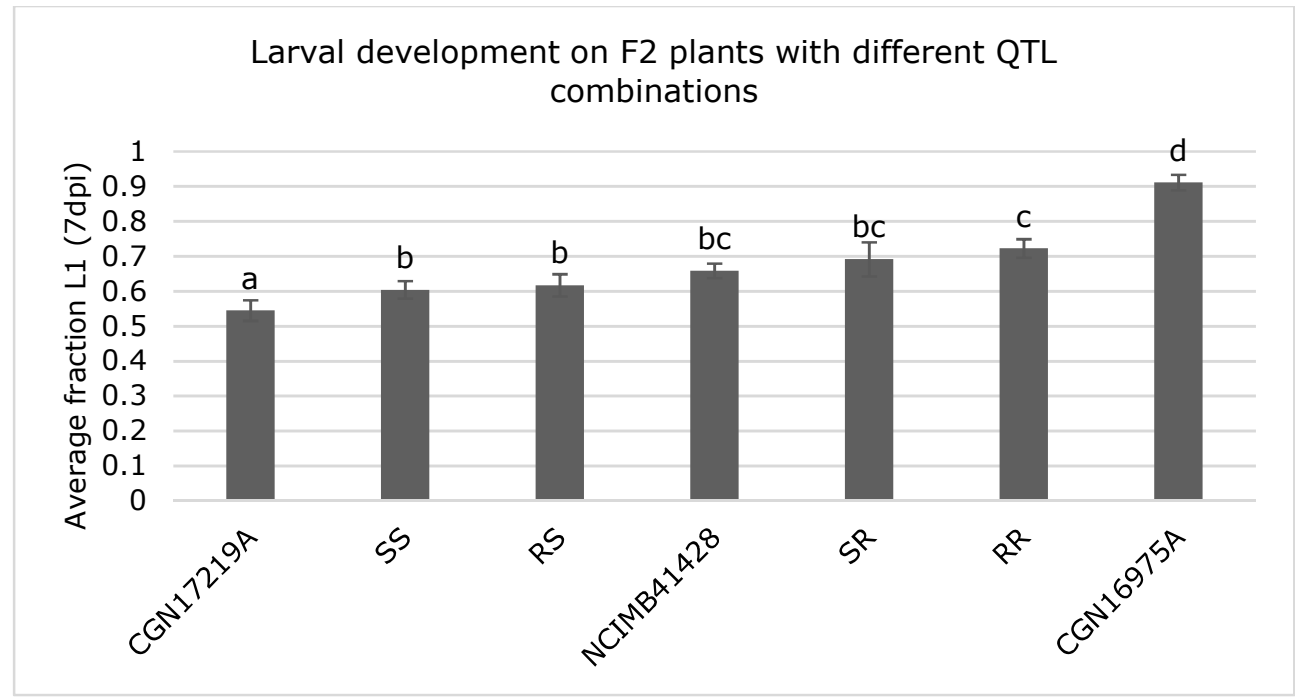

Figure 3: Larval development on $F 2$ plants derived from the cross between CGN17219A and NCIMB41428. The selected F2 plants have the resistance ("R") or susceptibility ("S") allele for the QTL region on chromosome 5 and 6 in homozygous state. The first " $R$ " or " $S$ " indicates the resistance or susceptibility allele for the QTL region on chromosome 5. The second " $\mathrm{R}$ " or " $\mathrm{S}$ " indicates the allele for QTL region on chromosome 6. The average fraction of L1 of plants with the same QTL-combination is shown. Bars sharing the same letter are not significantly different (Fisher's Protected LSD, P > 0.05). 
Larval development in two different Capsicum backgrounds (7dpi)

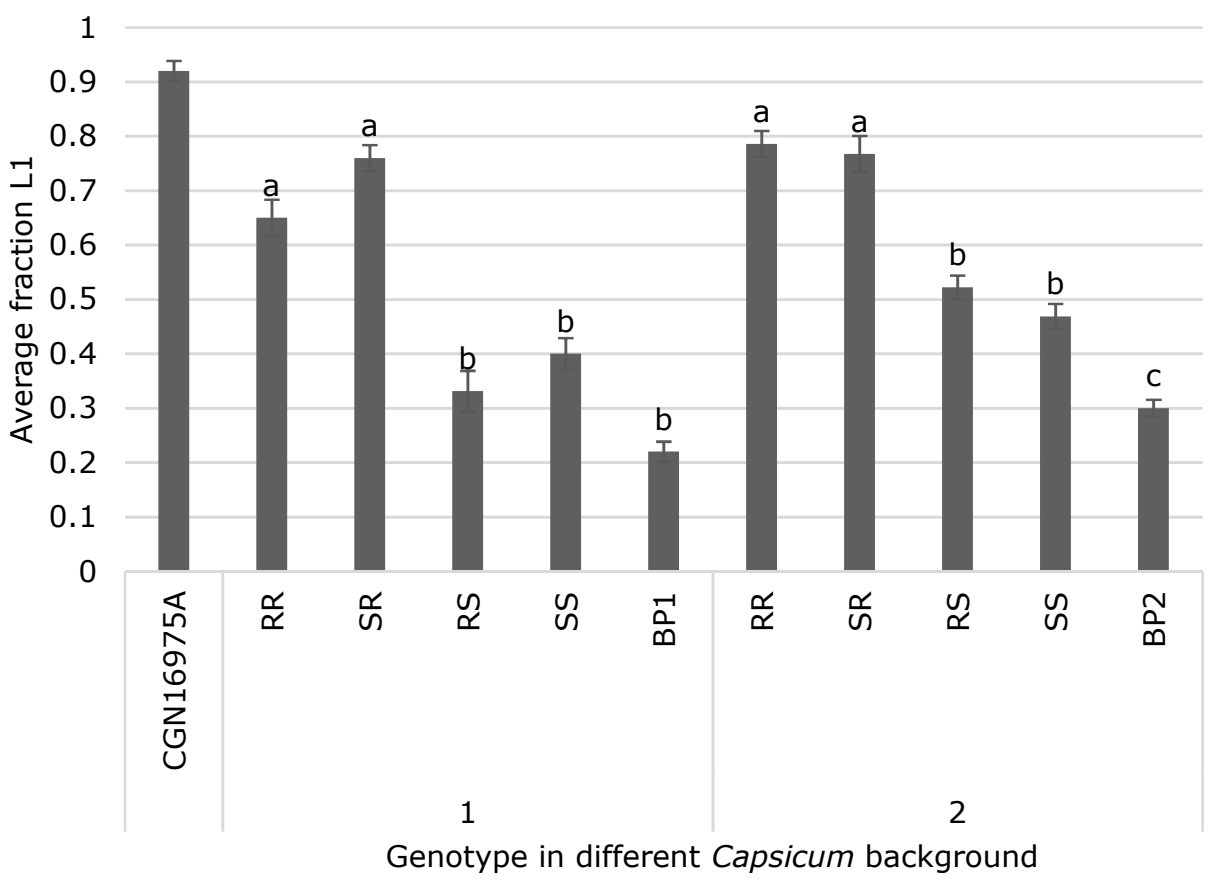

Figure 4: Larval development on plants that show all possible QTL-combinations in homozygous state for chromosome 5 and 6. Plants with two different Capsicum backgrounds were previously tested for $F$. occidentalis larval development in a detached leaf assay (Chapter 5 ). The 2-letter combination indicates whether the plants had the resistance allele ("R") or the susceptibility allele ("S") in homozygous state for the QTL regions on chromosome 5 (first letter) and 6 (second letter). A Fisher's LSD test was done to determine significant differences between plants with different QTL-combinations within the same background. Bars sharing the same letter within the same background are not significantly different (Fisher's Protected LSD, P > 0.05).

Figure 5 shows that a significant difference in silvering damage scores was found between BC3S2 plants with different QTL combinations for QTL regions on chromosome 5 and 6 ( $P<0.001$ ) (experiment 5). Plants with the resistance (" $R$ ") allele in homozygous state for the QTL region on chromosome 6 show a lower silvering damage score than plants that have the susceptibility (" $S$ ") allele in homozygous state for this region (Figure 5). We did not observe a significant difference between plants that have the " $\mathrm{R}$ " or " $\mathrm{S}$ " allele in homozygous state for the QTL region on chromosome 5. Overall, plants with genetic background 1 received a higher damage score than plants with genetic background 2 ( $P<$ $0.001)$. 


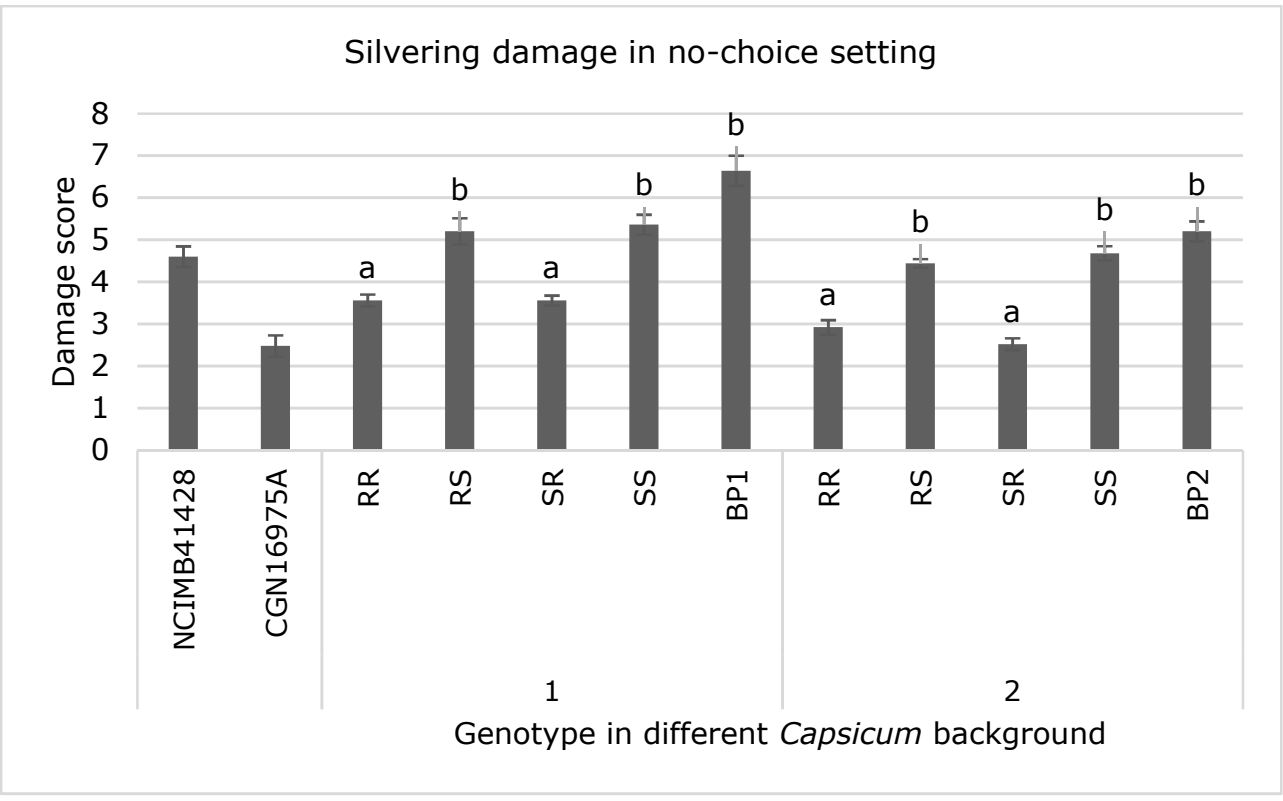

Figure 5: Average silvering damage scores of the five most damaged leaves of plants with different QTL combinations. The 2-letter combination indicates whether the lines had the resistance ("R") or susceptibility ("S") allele in homozygous state for chromosome 5 (first letter) and 6 (second letter). Average silvering damage scores are shown for plants from each Capsicum background separately. Error bars indicate the standard error. Average silvering damage scores followed with the same letter within the same background do not significantly differ (Dunn's post hoc test, $\mathrm{P}>0.05$ )

\section{Discussion}

\section{QTL on chromosome 6 contributes to thrips resistance}

In this study, we confirmed the effect of the QTL on chromosome 6 on pepper resistance to thrips. We have shown that plants with the resistance allele in homozygous state for the QTL region on chromosome 6 show stronger inhibition of larval development from the L1 into the L2 stage and less silvering damage compared to plants with the susceptibility allele in homozygous state. This corroborates the previously observed effect of the QTL on chromosome 6 on larval development and plant damage (Maharijaya et al., 2015). We also observed significant differences in larval development between plants from different F3 lines, that all have the susceptibility allele in homozygous state for the QTL region on chromosome 6. Plants from one of the F3 lines with the resistance allele in homozygous state for the QTL on chromosome 6 (Line 1) did not significantly differ in larval development from plants of another F3 line with the susceptibility allele for the same region (Line 4). Differences in levels of thrips resistance between lines with different genetic backgrounds have previously been observed in four Capsicum backgrounds and are further discussed in Chapter 5. The effect 
of the genetic background on thrips resistance shows the importance of determining the effect of QTLs in highly similar backgrounds, ideally in near isogenic lines (NILs), and also in different backgrounds.

\section{NCIMB41428 plants show intermediate levels of thrips resistance}

Both the larval development assay and the silvering damage assay showed that the plants from NCIMB41428 have a significantly higher level of thrips resistance compared to the susceptible reference CGN17219A, but a significantly lower level of thrips resistance than the resistant reference CGN16975A. Possibly, undetected additional minor QTLs that are present in CGN16975A are lacking in NCIMB41428, which might explain the intermediate level of resistance in NCIMB41428. In this respect it is a pity that we did not have the NCIMB41428 susceptible background to measure the true QTL effect. Also, we do not know whether the resistance in NCIMB41428 is actually caused by mechanisms underlying the QTL on chromosome 5, or whether NCIMB41428 also has the CGN16975 resistance allele for the QTL region on chromosome 6. In plants from the $\mathrm{F} 2$ population that resulted from a cross between CGN17219A and NCIMB41428, we observed a significant effect of the QTL on chromosome 6 on thrips resistance, although the differences in inhibition of larval development between groups of plants with different genotypes were small. Our observation that the QTL on chromosome 6 has a significant effect on thrips resistance suggests that plants from NCIMB41428 have the resistance QTL on chromosome 6 . However, this hypothesis has to be treated with caution as the flanking markers of the QTL on chromosome 6, that we used for the selection of the lines, were designed to distinguish the allele of the resistant $C$. annuum parent of the F2 population of Maharijaya et al. (2015) from the allele of the susceptible $C$. chinense parent. We do not know whether these markers can also distinguish the resistance allele of the $C$. annuum CGN16975 from the allele of the susceptible $C$. annuum parent of the cross from which NCIMB41428 was derived. Therefore, we can only conclude that the $C$. annuum allele in the QTL region on chromosome 6 has a significant effect on thrips resistance, but we cannot conclude whether this allele originated from the resistant parent CGN16975, or from the unknown susceptible C. annuum parent. It can also not be excluded that the CGN16975 accession contains multiple alleles at the chromosome $6 \mathrm{QTL}$, conferring different levels of resistance, and that line NCIMB41428 contains a less favourable allele than the parental plant used by Maharijaya et al (2015). 


\section{Effects of chromosome 5 QTL not confirmed in no-choice setting}

The effect of the QTL on chromosome 5 on thrips resistance could not be confirmed in this study, neither in the larval development assays nor in the silvering damage assays of the $\mathrm{F} 2$ population of the cross between CGN17219A and NCIMB41428, nor in the BC3S1 and BC3S2 plants in different backgrounds. Possible explanations are the use of a different bioassay, the use of different plant material with different genetic backgrounds, possible heterogeneity in the seed bank accession, and the use of different thrips populations, or a combination of these explanations.

Our resistance assay differed from the bioassay applied by Linders et al (2015), as we used a no-choice setting in our larval development and silvering damage assays, whereas Linders et al. (2015a) only used a damage assay in which the thrips could move between plants, thus a choice-setting. Therefore, plants with the NCIMB41428 resistance allele in homozygous state for the QTL on chromosome 5 might deter thrips (antixenosis), instead of affecting larval development or other life cycle parameters (antibiosis). Multiple cases of antixenotic resistance mechanisms against thrips have been reported. For instance, several tomato accessions have a moderate or strong antixenotic effect on 1-day-old $F$. occidentalis larvae, which seems to be related to the presence and density of trichomes (Krishna Kumar et al., 1995). Antixenotic effects on thrips have also been shown to Thrips palmi Karny in Phaseolus vulgaris L. accessions (Frei et al., 2003). In pepper, accessions with antixenotic effects on Aphis gossypii Glover, Myzus persicae Sulzer and Bemisia tabaci Gennadius have been identified (Bosland and Ellington, 1996; Ballina-Gomez et al., 2013; Latournerie-Moreno et al., 2015; Daryanto et al., 2016). This difference in bioassay method is a possible explanation why we could not confirm the effect of the QTL on chromosome 5 in this study, and why the two mapping studies of Maharijaya et al (2015) and Linders et al (2015) resulted in the detection of two different QTLs.

Another explanation why we could not confirm an effect of the QTL on chromosome 5 might be the role of the genetic background. We have shown that the genetic background has a significant effect on the levels of thrips resistance (Chapter 5). Key factors that are present in the initial mapping population of Linders et al. (2015) might be lacking in the backgrounds of the plant material that we used in this study, i.e. in the background of CGN17219A and in the two backgrounds of the $\mathrm{BC} 3 \mathrm{~S} 1$ and $\mathrm{BC} 3 \mathrm{~S} 2$ plants, thus resulting in a non-functional resistance mechanism located at chromosome 5 . If these key factors were not segregating in the mapping population of Linders et al. (2015), they could not have been detected and mapped. However, if the genetic background is to explain 
the difference, we would have expected to observe segregation of the background effects in the F2 population resulting from crosses between NCIMB41428 and CGN17219A, under the assumption that the QTL on chromosome 5 affects larval development in a non-choice setting, which we did not see.

Furthermore, the seed bank material from accession CGN16975 might be heterogenous. It could be that the plant that was used as a resistant parent for the mapping population of Linders et al. (2015) has a different allele for the QTL region on chromosome 5, compared to the plant that was used as a resistant parent for the mapping population of Maharijaya et al. (2015). However, this scenario implies that the QTL on chromosome 5 must have an effect on thrips resistance in the F2 population from a cross between NCIMB41428 and CGN17219A in this study, which we did not observe.

The use of different thrips populations might also explain why we could not confirm the thrips resistance QTL on chromosome 5. Although both studies used $F$. occidentalis, different rearing methods and different genetic backgrounds of the thrips might influence the preference of the insects or their performance on hosts. The rearing history of a thrips population may also affect the response on different genotypes. Karban (1989) observed that Apterothrips secticornis Trybom which had been reared on individual clones of their host seaside daisy (Erigeron glaucus), performed better on their native clone than on other clones. In pepper, Sun et al. (2018) show that 5 accessions that were resistant to Myzus persicae Sulzer reared on chinese cabbage, lacked a resistance response to the same Myzus population when the rearing was transferred to a susceptible pepper accession, thus they adapted to their host. As Linders et al. (2015) did not specify on which plant material the $F$. occidentalis population was reared, we cannot say whether the use of different rearing hosts might explain the different findings. Also, Visschers et al. (2019b) showed different levels of resistance to three $F$. occidentalis populations in pepper, suggesting the occurrence of local $F$. occidentalis biotypes with different genetic backgrounds. Our thrips biotype might have overcome the resistance mechanism underlying the QTL on chromosome 5, whereas the biotype used by Linders et al. (2015) to map this resistance QTL was sensitive to it. Similar observations have previously been made in the response of an aphid resistant Capsicum accession, in which the plant from the resistant accession provided resistance to a Dutch $M$. persicae population, but lacked a resistance response to a Swiss population of the same species (Sun et al., 2020). Also, it was shown that different $F$. occidentalis populations perform differently on resistant cucumber accessions (De Kogel et al., 1997a). Therefore, possible biotypic variation must be taken into account when screening for insect resistance. 


\section{Conclusions}

In this study, we aimed to find an explanation for the detection of different QTLS in two independent Capsicum mapping populations that use the same accession as resistance source. Furthermore, we aimed to determine the contribution of each QTL, or the combination of QTLs, to thrips resistance in Capsicum in nochoice settings, and to characterize the plant material from Linders et al. (2015). Although we did observe an intermediate level of thrips resistance in NCIMB41428, we could not confirm the effect of the QTL on chromosome 5 on thrips resistance in a no-choice setting. We hypothesize that NCIMB41428 has the resistance allele of the QTL on chromosome 6 derived from CGN16975. Our results show that the QTL on chromosome 6 contributes to thrips resistance in all tested Capsicum backgrounds. Therefore, this QTL shows potential for breeding thripsresistant varieties.

\section{Acknowledgement}

We thank Betty Henken for assistance in carrying out the detached leaf assay of the BC3S1 plants, and BASF Vegetable Seeds (Nunhems) for producing the plant material and for genotyping. This research was financially supported by a grant (TKI 1409-045) from the Ministry of Economic Affairs of the Netherlands and the breeding companies Bejo Zaden B.V. and BASF Vegetable Seeds (Nunhems).

\section{Author contributions}

$\mathrm{PvH}, \mathrm{BV}, \mathrm{REV}$ and JvL conceived and designed the experiments. PvH carried out the experiments, analysed the data and wrote the first draft of the manuscript. $\mathrm{BV}, \mathrm{REV}$ and JVL were involved in revision of the manuscript. 



\section{CHAPTER 7}

\section{sin 40}

(t)

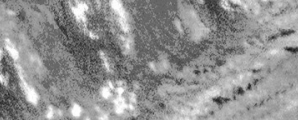

$=-\psi^{2}$
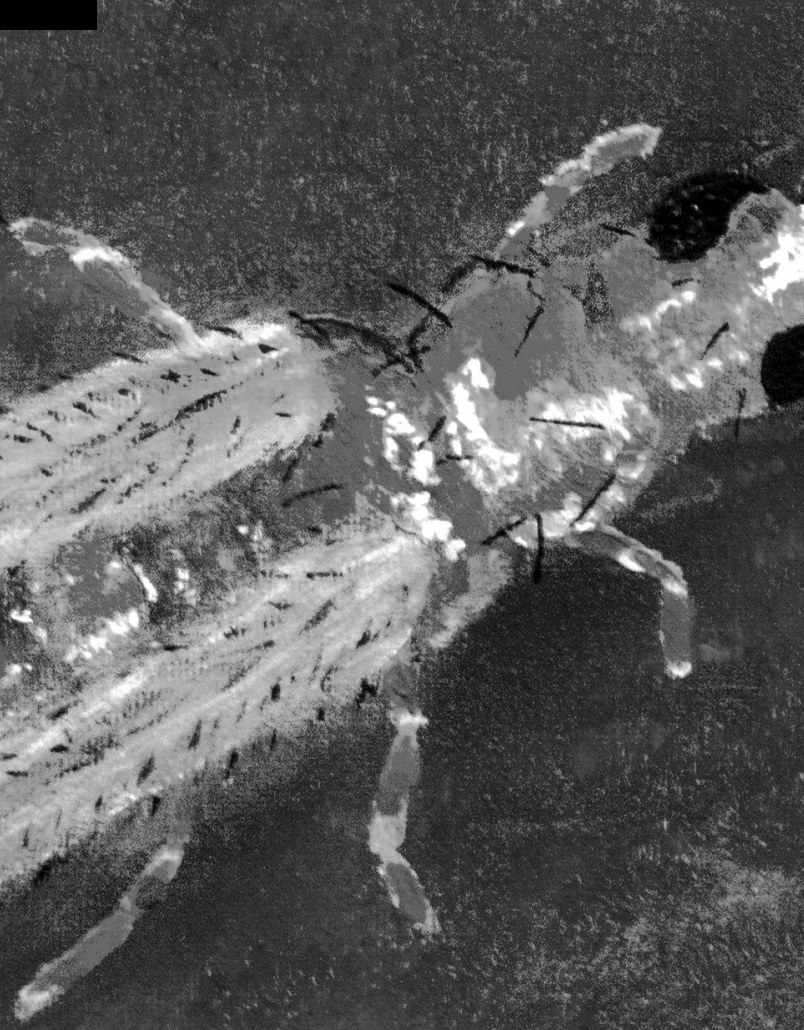

2).

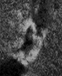

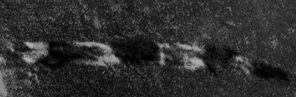


General discussion 
Thrips are a major pest in pepper cultivation, both in the field and in the greenhouse. They are difficult to control because of their high reproductive rate, short life cycle, cryptic behaviour and their wide host range (Hansen et al., 2003; Cloyd, 2009). Nowadays, protective measures have been taken to prevent thrips outbreaks in crops, mainly through applying monitoring, cultural, mechanical, chemical, physical and biological control measures (Mouden et al., 2017). These measures are only partially effective. Therefore, host plant resistance is a desired trait for pepper growers.

My thesis is a continuation of the work of Maharijaya (2013). His work focused on identifying accessions resistant to Frankliniella occidentalis (Pergande) and Thrips parvispinus (Maharijaya et al., 2011), characterizing the resistance factors (Maharijaya et al., 2012), mapping of resistance QTLs in an F2 population resulting from a cross between CGN16975 and CGN17219 (Maharijaya et al., 2015), and studying metabolites potentially involved in thrips resistance using a metabolite QTL (mQTL) approach (Maharijaya et al., 2019). The aims of my thesis were to answer the questions that remained, by focusing on further characterization of the resistance mechanism (Chapter 2), fine-mapping of the resistance QTL, and selecting candidate genes (Chapter $\mathbf{3}$ ), exploring the role of metabolites in the resistance mechanism encoded by genes located in the QTL on chromosome 6 (Chapter 4 ), studying the effect of the resistance QTL in different genetic backgrounds on two thrips species, thus its potential for breeding thripsresistant varieties (Chapter 5), and studying the effect on thrips resistance of the QTL on chromosome 5 that was previously identified by Linders et al. (2015a) (Chapter 6). In this chapter, I will discuss the main findings in the light of elucidating the resistance mechanism, suggestions for improved thrips resistance research, and how my findings contribute to breeding for thrips resistance.

\section{Elucidating the resistance mechanism of CGN16975}

Resistance may affect the interaction between plant and insect in different ways. Depending on the resistance mechanism, these can be divided in antibiosis, antixenosis and tolerance. Antibiotic mechanisms affect the life history parameters of thrips, for instance by delaying larval development or by affecting their survival (Smith, 2005). Antixenotic mechanisms affect the behaviour of thrips, for instance by producing odours or chemicals that deter thrips from feeding (Smith, 1989). The resistance mechanism encoded by genes located in the QTL on chromosome 6 affects larval development from the first instar into the second instar larval stage, therefore this is an example of antibiosis-type resistance (Maharijaya et al., 2012). Studies on the characterization of the resistance mechanism have shown that larval development is inhibited on young leaves of the resistant accession CGN16975 (Chapter 2). My fine-mapping study combined with RNA 
sequencing resulted in the selection of three candidate genes that might play a role in inhibition of larval development, i.e. acid phosphatase 1 (APS1), organic cation transporter 7 (OCT7) and an uncharacterized locus (LOC107874801) (Chapter 3). Acid phosphatase activity has been previously linked to insect resistance (Mason and Mullet, 1990; Berger et al., 1995; McConn et al., 1997; Stotz et al., 2000; Berger et al., 2002; Reymond et al., 2004; Liu et al., 2005). Organic cation transporters might be involved in insect resistance as well, as the concentration of one of the compounds that can be transported by OCT, trigonelline, was significantly lower in thrips-resistant tomato accessions (Mirnezhad et al., 2010). Further fine-mapping of the resistance QTL is difficult due to the small genetic distance and requires the development of additional markers. Which gene or genes is or are conferring thrips resistance is currently unknown. My metabolomics approach showed that diterpene glycosides do not play a role in the resistance mechanism in our plant material (Chapter 4 ). This is an unexpected finding, as two studies have shown that diterpene glycoside abundance is associated with thrips resistance in Capsicum (Macel et al., 2019; Maharijaya et al., 2019). Diterpene glycosides were also associated with resistance to the mirid (Tupiocoris notatus Distant) and to larvae of tobacco hornworm (Manduca sexta Linnaeus) or other specialist herbivore larvae in Nicotiana attenuata (Jassbi et al., 2006; Heiling et al., 2010; Dinh et al., 2013). I did not identify other metabolites that might play a role in the thrips resistance mechanism encoded by one of the fifteen genes located in the resistance QTL; either because my extraction and LC-MS method could not detect the relevant metabolite(s), or because metabolites do not play a role in the resistance mechanism. Next to a further characterization of the mechanism, I have also shown that the resistance QTL in CGN16975 is effective against two thrips species, i.e. F. occidentalis and Thrips tabaci (Lindeman) in four Capsicum backgrounds (Chapter 5), and that the QTL on chromosome 6 works independently from the previously described thrips resistance QTL on chromosome 5 (Chapter 6).

The resistance QTL was fine-mapped to a region harbouring fifteen predicted genes. However, I do not know whether I have identified all genes in the QTL region of the resistant accession CGN16975, as there may be differences between this accession and the accessions that were used to assemble the reference genomes. In my study, I used the Zunla assembly (Qin et al., 2014) and the UCD10X assembly (Hulse-Kemp et al., 2018) to map the RNA reads in order to study differential gene expression and to detect SNPs that are predicted to lead to changes in protein structure that might result in disruption of function or changed affinity to a ligand. I designed additional markers to narrow down the region of interest in order to identify the causal gene. Differences between the Zunla and UCD10X assemblies can be found in the QTL region for thrips resistance 
on chromosome 6, including different predicted genes, different physical distances between markers, and different marker locations. This problem may be addressed by obtaining a de novo long read sequence of the resistant accession (CGN16975) of high coverage and mapping the RNA reads to this sequence. This would provide a more detailed overview of the region under investigation, including potential insertions or deletions that were not present in the currently available reference genomes.

To identify the causal gene, a suitable approach would be to knock out the candidate gene using the CRISPR-Cas technique. If the candidate gene is a susceptibility gene, it is expected that a knock-out of the candidate gene in a susceptible accession will lead to increased thrips resistance in the mutant plants. If the candidate gene confers resistance, a knock-out of the gene in the resistant accession is expected to lead to susceptibility. Transformation and regeneration of Capsicum has been studied intensively the past decades, and it was shown that regeneration of Capsicum is troublesome and accession-specific (Gunay and Rao, 1978; Díaz et al., 1988; Agrawal et al., 1989; Valera-Montero and Ochoa-Alejo, 1992; Peddaboina et al., 2006; Sanatombi and Sharma, 2008; Valadez-Bustos et al., 2009; Kumar et al., 2012). A knock-out of candidate genes can also be obtained through treating seeds with ethyl methanesulfonate (EMS), thus introducing mutations. Multiple studies have shown the possibilities of induced mutations in Capsicum through application of EMS in different concentrations and durations (Alcantara et al., 1996; Subhash et al., 1997; Arisha et al., 2015). By using EMS rather than the CRISPR-Cas approach, regeneration of Capsicum explants is not necessary to obtain mutants. However, CRISPR-Cas has the advantage of targeted mutagenesis, while treatment with EMS also causes mutations in the background outside of the target regions, leading to potential unexpected effects on the phenotype. Through backcrossing, mutations outside of the target region can be limited in next generations, while keeping the mutation(s) in the QTL region that might lead to changes in thrips resistance.

Introduction of a candidate gene into a susceptible Capsicum accession can also be an approach to validate candidate genes, if the candidate gene confers resistance. Agrobacterium tumefaciens-mediated transformation of explants in pepper has been used before in Capsicum. However, efficiencies are low. In order to avoid the recalcitrant regeneration in Capsicum, particle bombardment of pollen may be used to obtain transgenic plants without the need to regenerate plants (Sanford et al., 1987; Klein et al., 1992; Twell et al., 1997; Eapen, 2011; Zhao et al., 2017).

The study of gene expression in thrips-exposed and mock-treated F4 plants showed that some genes are differentially expressed at six hours after thrips 
infestation (Chapter 3). In this study, I only included one time point, thus genes that are differentially expressed before or after this time point were missed in our analysis. Therefore, a thorough time series of thrips resistance response in the F4 plants that either have the resistance or susceptibility allele in the QTL region on chromosome 6 should be made to determine the gene expression in leaves of both groups of plants at different time points after infestation, and compare these to mock-treated leaf samples. A study of the response of a susceptible sweet pepper response to $F$. occidentalis (Sarde, 2019) shows that changes in gene expression may be missed if certain time points are not included in the time series. Comparing gene expression between resistant and susceptible F4 plants for both treatments can give more insight in whether the gene or genes that play a role in the resistance mechanism, and which are located in the resistance QTL, are induced or constitutively expressed, and whether they are resistance or susceptibility genes. This can be used as a starting point to further elucidate the resistance mechanism.

\section{Targets for future thrips resistance research}

In this thesis, I used a multidisciplinary approach to study thrips resistance in Capsicum. RNA sequencing provided not only insight in expression of genes located in the QTL area, it also enabled me to identify SNPs that were predicted to lead to structural changes in proteins (Chapter 3); I used this for the selection of candidate genes. My metabolomics approach showed that diterpene glycosides do not play a role in the resistance mechanism in my plant material (Chapter 4). Combining these different approaches in one study provided a powerful tool to explore the QTL region and select candidate genes, although the candidate genes still need to be validated. Multidisciplinary approaches to study insect resistance have previously resulted in the selection of candidate genes in resistance to cabbage whitefly (Broekgaarden et al., 2018) and in resistance to two whitefly species in tomato (Vosman et al., 2018; Vosman et al., 2019).

In this thesis, I compared gene expression and metabolite profiles in leaves infested with L1 larvae (Chapter 3 and 4). I observed differences in gene expression between infested and mock-treated leaves suggesting that the infestation with L1 larvae leads to a response in plant cells. This could indicate that a resistance mechanism is induced upon thrips feeding, but further analysis of differential gene expression should be done before a conclusion on whether the resistance is induced or constitutively expressed can be drawn. Also, this approach did not allow a conclusion on whether the cells next to cells that have been probed by thrips react differently than cells further away of the feeding site. Therefore, it would be interesting to add a spatial component. As thrips ingest cell content, studying the plant's response in the exact targeted cells seems impossible. 
However, single cell sequencing technology (Tang et al., 2009; Shapiro et al., 2013) could be used to sequence the neighbouring cells of the target cell to study the cell-specific response. The hardest part is to identify and isolate neighbouring cells at different time points after the thrips probe the target cell, in order to capture the resistance response in neighbouring cells to thrips feeding. If the resistance mechanism is induced upon thrips infestation, it would be interesting to identify genes that are differentially expressed to further elucidate the resistance mechanism and the plant's response to thrips.

Also, it has been shown that the saliva of several herbivorous insects contains effector proteins that manipulate the plant's response to an attack (Hogenhout and Bos, 2011; Mugford et al., 2016; Villarroel et al., 2016). However, effector proteins in thrips saliva have not been characterized yet. Therefore, it would be interesting to analyse and identify the different proteins present in thrips saliva, and to study gene expression in the thrips salivary gland, to predict effector proteins, isolate them from the saliva or produce them synthetically, and study their effect on the resistant and susceptible host plant. A similar approach has resulted in the identification, and in some cases validation, of putative effector proteins in aphids (Harmel et al., 2008; Bos et al., 2010; Carolan et al., 2011; Cooper et al., 2011; Elzinga et al., 2014). When these effector proteins can be identified and isolated from thrips saliva, probing can be mimicked mechanically by using a stylet shaped device and simultaneously apply effector proteins. This way, the exact target site can be chosen, thus this could be a first step towards studying the response to (mimicked) thrips probing in neighbouring cells, and the systemic response in cells at a specified distance from the targeted cell.

Studying an interaction between two living systems, i.e. thrips and pepper in this thesis, requires at least two different point of views. In my thesis, I focused on the plant's response to thrips. It was shown that larval development from the first into second instar larval stage (L1 into L2 stage) was impaired (Maharijaya et al., 2012), but it is not known how this developmental process is blocked. There are several possibilities that could explain the lack of larval development on resistant plants. It could be that larval development is blocked because the L1s are not able to obtain essential nutrients or ingest plant compounds that are toxic or become toxic in their digestive system (antibiosis), or that L1s are repelled from the plant and refuse to eat (antixenosis) even in a no-choice situation. One of the candidate genes in this thesis, encoding acid phosphatase, has been shown to cause high mortality and delays in larval development when added to the diet of insects with an acidic gut lumen, like thrips (Annadana et al., 2002; Outchkourov et al., 2004; Liu et al., 2005). The other candidate gene identified in this thesis, which encodes an organic cation transporter, was previously shown to affect expression in 
carnitine-related genes in Arabidopsis. Carnitine is an essential nutrient for larvae from some insect species, e.g. Tenebrio larvae (Fraenkel, 1953; Lelandais-Brière et al., 2007). Metabolomics targeted to compounds that are transported by the organic cation transporter could provide more insight in the potential role of this gene in thrips resistance.

Moreover, in my study, I used thrips that were not infected with tospoviruses such as tomato spotted wilt virus (TSWV). However, it has been shown that feeding behaviour of TSWV-infected thrips differs from uninfected thrips. For instance, a study by Stafford et al. (2011) showed that male thrips infected with TSWV made almost threefold more non-ingestion probes compared to uninfected male thrips. During these probes thrips salivate, but leave the cells mostly intact. TSWV infection requires functioning cells, thus the increase of non-ingestion probes increases virus transmission. Also, it has been shown that thrips are more attracted to virus-infected plants compared to uninfected plants (Maris et al., 2004; Belliure et al., 2005). Female thrips laid more eggs on TSWV-infected compared to uninfected plants, and the time required to develop from egg to pupal stage was significantly decreased in infected plants (Maris et al., 2004). It was hypothesized that viruses can help thrips to overcome plant defences to promote further spread (Belliure et al., 2005). The ability of pathogens to alter insect behaviour has been shown in other pathogen-insect interactions as well (Molyneux and Jefferies, 1986; Friedli and Bacher, 2001; Eigenbrode et al., 2002; Johnson et al., 2003; Schaub, 2006). Therefore, it will be interesting and relevant to confirm the effect of the resistance QTL using thrips infected with TSWV.

\section{Prospects for breeding thrips resistance varieties}

Fine-mapping the resistance QTL and characterizing and elucidating the resistance mechanism are important steps towards breeding for thrips resistance. In order to prevent that the selection of the resistance trait leads to the undesired selection of other traits (linkage drag), the region of interest should be narrowed down as much as possible, preferably to the gene(s) conferring resistance. Further elucidation of the resistance mechanism can give insight on the genetic region that is needed to confer resistance to susceptible varieties, without the risk of selecting undesired traits. By fully understanding the genetic basis of thrips resistance, this information can be used to breed for thrips resistance in pepper. For instance, the resistance mechanism may be stacked with other resistance mechanisms to confer more durable resistance. Knowing their genetic basis enables breeders to combine these mechanisms without the risk that they interfere with each other. Also, identification of genes that play a role in the resistance mechanism can result in a targeted approach to study homologs of the identified genes and their role in thrips resistance in other plant species. If the 
causal gene can be identified, this mechanism may also be transferred to other Solanaceae species. It has been shown that the transfer of a resistance gene to a susceptible related species can complement the susceptible phenotype: for instance, a resistance $(R)$ gene that confers high levels of resistance against late blight in potato was successfully transferred to a susceptible tomato background, and complemented the susceptible phenotype (Van Der Vossen et al., 2003). Another example is the transfer of a pepper gene conferring resistance to root knot nematodes to susceptible tomato plants, which resulted in increased levels of resistance (Chen et al., 2007).

In this thesis, I have shown that the resistance QTL on chromosome 6 is suitable for breeding thrips- resistant varieties. My study showed that the expression of the resistance starts at a plant age between 4 and 8 weeks, and the resistance is expressed to a significantly higher extent in the youngest fully opened leaves compared to older leaves, thus providing information for integrated pest management and crop protection (Chapter 2). My whole plant damage assay and oviposition assay showed that the resistance expression in young leaves has a significant effect on thrips damage, thus showing its potential. However, it is important that extra protective measures are taken until the pepper plants reach the plant age at which the resistance is fully expressed, for instance through application of biological control. I have shown that the QTL affects larval development in both F. occidentalis and T. tabaci (Chapter 5), which indicates that the resistance mechanism is effective against several thrips species. As the resistant accession CGN16975 was initially identified using $T$. parvispinus (Maharijaya et al., 2011), it would be interesting to determine whether the QTL also affects $T$. parvispinus and thrips species other than $F$. occidentalis and $T$. tabaci that are pests in pepper cultivation. Also, I showed that the resistance QTL is effective in different genetic backgrounds (Chapter 5). Significant differences in thrips resistance between different genetic backgrounds were observed, which indicates that other factors in the genetic background enhance or impede thrips resistance. This finding supported the finding of Maharijaya et al. (2015). They observed high heritability for thrips resistance, which indicates that the genetic variance that was not explained by the QTL (around $50 \%$ ) is most likely due to other genetic factors. Further research on these genetic factors could also give more insight in the resistance mechanism, either related or unrelated to the identified QTL. When these factors are identified, their effect could be assessed, and it might be possible to target them in breeding for thrips resistance. Also, I have shown that the resistance QTL on chromosome 6 works independently from the resistance QTL previously mapped to chromosome 5 by Linders et al. (2015) (Chapter 6). Altogether, these findings indicate that the resistance QTL on chromosome 6 can be used for breeding thrips-resistant varieties. 
As mapping of the resistance resulted in one QTL that explained about $50 \%$ of the genetic variance in the $\mathrm{F} 2$, it is not unlikely that only one gene, or a limited number of genes provide the key to acquiring thrips resistance. It is most likely that the other $50 \%$ of variance is explained by other resistance factors in the genetic background that could not be mapped in the population of Maharijaya et al. (2015). These genetic factors may be lost when breeders only target the QTL region. This means that thrips may only need to adapt to one gene in order to overcome the resistance. In order to acquire a more durable resistant variety, it is therefore advised to stack different resistance mechanisms, either mechanisms targeting another developmental stage of the thrips, or mechanisms that affect thrips through different, preferably independent, pathways. In order to find these QTLs, Capsicum accessions should be screened with different phenotyping methods targeting different life stages. The QTL on chromosome 6 specifically targets $L 1$ into $L 2$ development, thus it would be desired to stack this resistance with mechanisms targeting for instance thrips oviposition or adult survival. Even when the QTL has a minor effect on thrips resistance, the combination of different resistance mechanisms can reduce the risk that thrips overcome the resistance. Other resistance mechanisms targeting other thrips life stages than molting from L1 into L2 have been identified in other crops. For instance, it has been shown that acyl sugars have a negative effect on thrips oviposition in tomato (BenMahmoud et al., 2018). Also, it has been suggested that compounds excreted by glandular trichomes (type I and/or IV) in tomato negatively affect adult thrips survival (Vosman et al., 2018). In most studies on thrips resistance, the exact mechanism remains unknown or hypothesized mechanisms need to be validated (Frei et al., 2005; Omo-Ikerodah, 2008; Mirnezhad et al., 2010; Muchero et al., 2010; Maharijaya et al., 2015; Sobda, 2017; Vosman et al., 2018; Macel et al., 2019; Visschers et al., 2019b). Therefore, it would be relevant to further elucidate these mechanisms to determine whether combining these mechanisms can be used to breed durable thrips-resistant Capsicum varieties.

\section{Combining thrips resistance with virus resistance}

Next to stacking different resistance mechanisms to thrips in resistant varieties, it will be beneficial to include resistance to tospoviruses such as tomato spotted wilt virus (TSWV). Thrips larvae acquire the virus from an infected plant in the L1 or early L2 larval stage (Moritz et al., 2004). The virus replicates in the thrips, and can only be reintroduced to uninfected plants when thrips reach the late L2 or adult stage (Whitfield et al., 2005). Adult thrips remain viruliferous for life (Jones, 2005). In the resistant accession CGN16975, or plants that have the resistance allele in homozygous state for the QTL region, L1 larvae might be able to acquire the virus, but as the majority does not develop into the next stage, the risk of virus transmission to other plants is limited. In this way, the resistance QTL is 
very suitable to reduce the pressure on virus resistance mechanisms. In pepper, one resistance gene, TSW, that confers resistance to TSWV has been identified (Boiteux and de Ávila, 1994), but it has been shown that the resistance can be overcome by certain TSWV isolates when temperature rises above $28^{\circ} \mathrm{C}$ (Ronde et al., 2019). The resistance gene has been mapped to chromosome 10 (Jahn et al., 2000). This means Tsw can be combined with the thrips resistance QTL on chromosome 6 , to create varieties that are better protected during TSWV outbreaks.

\section{Conclusion}

In this thesis, I have characterized and fine-mapped the resistance mechanism of a resistant Capsicum annuum accession CGN16975. On this resistant accession, larval development from first into second instar larvae is inhibited. I have shown that the resistance mechanism starts being expressed at a plant age between 4 and 8 weeks. The resistance is expressed to significantly higher extent in the youngest fully opened leaves, but I have shown that this resistance mechanism is relevant as oviposition mainly occurs on the youngest leaves, and whole plant damage assays showed limited thrips damage on the resistant accession. Next, fine-mapping of the resistance QTL combined with a RNAseq approach led to the identification of three candidate genes, i.e. APS1, OCT7 and LOC107874801. The metabolomics approach showed that diterpene glycosides do not play a role in this resistance mechanism. Furthermore, I showed that the resistance QTL is effective against two thrips species in four different Capsicum backgrounds, and that this QTL works independently from the reported thrips resistance QTL on chromosome 5. Approaches to identify the causal gene and suggestions for future directions in thrips resistance breeding have been discussed. Altogether, I have shown that the resistance QTL on chromosome 6 can be used to breed thripsresistant varieties. 




\section{Summary}

Thrips are small insects that are a major pest in Capsicum cultivation. They feed on fruits, leaves and flowers, leaving silvering scars, and leading to plant organ deformation, reduced photosynthetic capacity, altered carbon allocation, reduced fruit set, and thus reduced yield. Thrips also indirectly damage plants through the transmission of tospoviruses such as tomato spotted wilt virus (TSWV). Pepper growers use protective measures such as biological and chemical control, but these measures only limit thrips population growth partially due to their high reproductive rate, short life cycle, cryptic behavior and evolved insensitivity to insecticides. Therefore, growers would benefit from host plant resistance.

Previously, resistant Capsicum accessions have been identified. It has been shown that Frankliniella occidentalis larval development from the first into the second instar larval (L1 into L2) stage was inhibited on the youngest fully opened leaves of 12 week old pepper plants. A resistance quantitative trait locus (QTL) that originated from the resistant accession CGN16975 has been mapped to chromosome 6 in an $\mathrm{F} 2$ population. In an independent study a resistance QTL was mapped to chromosome 5 in a population derived from the same resistant, but a different susceptible parent. Furthermore, six metabolite QTLs have been identified that co-locate with the resistance QTL on chromosome 6, thus these metabolites might play a role in the resistance mechanism encoded by genes located in the resistance QTL. Two of these metabolites are diterpene glycosides, which are compounds that were previously shown to correlate to thrips resistance in Capsicum.

In this thesis, I aimed to further characterize the resistance mechanism in CGN16975 and its potential as a source for breeding for thrips-resistant varieties. The resistance QTL was fine-mapped and a multidisciplinary approach was employed to select candidate genes. Furthermore, the role of metabolites in the thrips resistance mechanism encoded by genes located in the QTL on chromosome 6 was investigated.

The effect of plant development on thrips resistance in Capsicum has been evaluated. The fraction $L 1$ that did not develop into $L 2$ was used as a measure for thrips resistance. The resistance levels in the youngest fully opened leaves of three resistant, three intermediate resistant and three susceptible accessions at a plant age of 4,8 and 12 weeks were assessed. It was shown that thrips resistance starts being expressed at a plant age between 4 and 8 weeks. Furthermore, inhibition of larval development on leaves from 5 different leaf age classes from one resistant (CGN16975) and one susceptible (CGN17219) accession was quantified. In the resistant accession, the level of thrips resistance was 
significantly higher in the youngest fully opened leaves compared to older leaves, while in the susceptible accession, L1s performed significantly better on the youngest leaves compared to older leaves. Although the resistance is not equally expressed in all plant parts, the significantly reduced silvering damage in the whole plant damage assay, and the preference of female adults to lay their eggs in younger compared to older leaves showed that the resistance mechanism in CGN16975 is relevant and effective, thus that this source can be used in breeding thrips-resistant Capsicum varieties.

The resistance QTL on chromosome 6 has been fine mapped to a region of 0.4 Mbp harboring 15 genes. Two of these genes, i.e. organic cation transporter 7 (OCT7) and uncharacterized locus LOC107874801, showed altered expression upon L1 infestation in F4 plants that have the resistance allele for the QTL region in homozygous state, compared to F4 plants that have the susceptibility allele in homozygous state. Three genes, i.e. acid phosphatase 1 (APS1), OCT7 and LOC107874801, had a SNP that was predicted to lead to structural changes in proteins. Therefore, APS1, OCT7 and LOC107874801 are the most likely candidate genes playing a role in the thrips resistance mechanism encoded by genes located in the resistance QTL.

The role of diterpene glycosides and other metabolites in the thrips resistance mechanism underlying the resistance QTL on chromosome 6 was investigated. The relative metabolite abundance in plants from the resistant accession CGN16975 and susceptible accession CGN17219, as well as between F4 plants that have the resistance allele and susceptibility allele in homozygous state for the QTL region, for two treatments (i.e. exposed to L1 and mock-treated), was determined. Thirty-six putative diterpene glycosides were found that occurred in significantly different abundance between the resistant and susceptible accession. Eighteen putative diterpene glycosides were significantly more abundant in the resistant accession, the other eighteen were more abundant in the susceptible accession. No significant differences in putative metabolite abundances were observed between the different groups of F4 plants for both treatments. From this analysis, it can be concluded that diterpene glycosides do not play a role in the resistance mechanism encoded by genes located in the QTL on chromosome 6.

The effect of the resistance QTL on chromosome 6 on resistance to two thrips species, i.e. F. occidentalis and Thrips tabaci, when introgressed into four susceptible Capsicum backgrounds was assessed. Inhibition of larval development was compared in plants that have the resistance allele in homozygous state for the QTL region, to plants that have the susceptibility allele in homozygous state, within the same genetic background. Although significant differences in thrips resistance level between different backgrounds were observed, indicating that 
genetic factors in the different backgrounds affect thrips resistance, I showed that the QTL affected larval development in two thrips species in all tested backgrounds. This finding shows that the QTL can be applied in breeding thripsresistant Capsicum varieties.

The contribution of each of the two thrips resistance QTLS, or a combination of both QTLs, to thrips resistance in a no-choice setting was assessed. I observed an intermediate level of thrips resistance in plants of the near-isogenic line (NIL) that was filed with the patent application in which the QTL on chromosome 5 was described. The QTL on chromosome 5 did not affect thrips resistance in both larval development and silvering damage assays. In contrast, a significant effect of the QTL on chromosome 6 was found on both larval development and on silvering damage. I hypothesize that the NIL that was filed with the patent application has the resistance allele on chromosome 6 that originated from CGN16975. The findings from this study show that the QTL on chromosome 6 works independently from the QTL on chromosome 5 in all tested backgrounds.

In the general discussion I review approaches to identify the causal gene(s) conferring resistance to thrips, and future directions of breeding for thrips resistance in pepper. Altogether, the work in this thesis contributes to protect pepper against thrips; first, by providing insight in the effect of plant- and leaf age on thrips resistance that can be used to improve integrated pest management strategies; second, by fine mapping the resistance QTL to a region of $0.4 \mathrm{Mbp}$, which enables breeders to introgress the resistance QTL with limited risk of linkage drag; third, by selection of candidate genes located in the resistance QTL, and thus providing a good starting point for identification of the causal gene(s), which might enable the transfer of the resistance mechanism to other susceptible Solanaceae; and lastly, by confirming the effect of the QTL in different backgrounds to two thrips species, which shows that this QTL can be used as a resistance source for breeding thrips-resistant Capsicum varieties. 



\section{Samenvatting}

Tripsen zijn kleine insecten die grote problemen veroorzaken in de teelt van Capsicum (paprika en peper). Ze zuigen hun voeding uit de vruchten, bladeren en bloemen, en veroorzaken daardoor een zilverachtige schade, en dit leidt tot misvormde plantorganen, verminderde fotosynthetische capaciteit, een veranderde koolstofallocatie, verminderde vruchtvorming, en dus verminderde opbrengst. Tripsen kunnen ook indirect schade toebrengen aan planten door het overbrengen van tospovirussen zoals tomatenbronsvlekkenvirus (TSWV). Telers gebruiken beschermende maatregelen zoals biologische en chemische bestrijdingsmiddelen, maar deze middelen kunnen de groei van de tripspopulatie slechts gedeeltelijk inperken, door de snelle voortplanting, korte levenscyclus, het verschuilgedrag van tripsen, en doordat ze ongevoeligheid voor pesticiden kunnen ontwikkelen. Daarom zijn telers gebaat bij de ontwikkeling van tripsresistente Capsicum-rassen.

In eerdere onderzoeken zijn Capsicum-accessies gevonden die resistent zijn tegen tripsen. Daarin is aangetoond dat de ontwikkeling van de Californische trips, Frankliniella occidentalis, van het eerste naar het tweede larvale stadium (L1 naar L2) wordt onderbroken op het jongste volledig geopende blad van 12 weken oude resistente Capsicum-planten. Een resistentie-QTL (quantitative trait locus), oorspronkelijk afkomstig uit Capsicum-accessie CGN16975, werd gekarteerd op chromosoom 6 in een $\mathrm{F} 2$ populatie. In een andere onafhankelijke studie werd een resistentie-QTL gekarteerd op chromosoom 5 in een populatie met dezelfde resistente, maar een andere vatbare ouder. Daarnaast zijn er zes metabolietenQTLs geïdentificeerd die samenvielen met het resistentie-QTL op chromosoom 6, dus deze metabolieten zouden een rol kunnen spelen in het resistentiemechanisme dat wordt gecodeerd door de genen in het QTL-gebied. Twee van deze metabolieten zijn diterpeenglycosiden. Dit zijn stoffen die ook al in eerdere onderzoeken een correlatie lieten zien met trips-resistentie in Capsicum.

Het doel in deze thesis is om het resistentiemechanisme uit accessie CGN16975 verder te karakteriseren, en te onderzoeken of het kan worden gebruikt om tripsresistente Capsicum-rassen te ontwikkelen. Het resistentie-QTL is fijngekarteerd en een multidisciplinaire aanpak is gebruikt om kandidaatgenen te selecteren. Daarnaast is de rol van metabolieten in het trips-resistentiemechanisme dat gecodeerd wordt door genen in het QTL-gebied op chromosoom 6 onderzocht.

Het effect van plantontwikkeling op trips-resistentie in Capsicum is getoetst. De fractie L1 die niet doorontwikkelde naar het L2 stadium is gebruikt om het resistentieniveau te bepalen. De resistentieniveaus in de jongste volledig 
geopende bladeren van drie resistente, drie intermediaire en drie vatbare accessies werden bepaald op een plantleeftijd van 4, 8 en 12 weken. Hierbij is aangetoond dat trips-resistentie tot expressie kwam in planten met een leeftijd tussen 4 en 8 weken. Daarnaast zijn de resistentieniveaus van bladeren van de resistente accessie CGN16975 en de vatbare accessie CGN17219 bepaald voor 5 verschillende bladleeftijden. Het resistentieniveau van de bladeren van de resistente accessie was significant hoger op het jongste blad vergeleken met de oudere bladeren, terwijl de L1 op de vatbare accessie juist het best presteerden op de jongste bladeren. Ondanks dat de resistentie dus niet in alle delen van de plant in gelijke mate tot expressie komt, laten de verminderde zilverachtige schade in een schadetoets met de hele plant en de voorkeur van volwassen tripsvrouwtjes om hun eieren op jonge in plaats van oudere bladeren te leggen zien dat het resistentiemechanisme van CGN16975 relevant en effectief is, dus dat deze bron kan worden gebruikt om tripsresistente peperrassen te ontwikkelen.

Het resistentie-QTL op chromosoom 6 is fijngekarteerd tot een gebied van 0.4 Mbp waarin 15 genen liggen. Twee van deze genen, namelijk organic cation transporter 7 (OCT7) en een ongekarakteriseerd locus LOC107874801, verschilden in genexpressie wanneer planten met het resistentie-allel homozygoot aanwezig in het QTL-gebied werden geïnfesteerd met L1s, vergeleken met planten met het vatbaarheidsallel homozygoot aanwezig. Drie genen, namelijk acid phosphatase 1 (APS1), OCT7 en LOC107874801, hadden een single nucleotide polymorphism (SNP) die mogelijk in een verandering in eiwitstructuur resulteren. Om deze redenen zijn APS1, OCT7 en LOC107874801 de meest aannemelijke kandidaatgenen om een rol te spelen in het trips-resistentiemechanisme dat wordt gecodeerd door een gen of genen in het resistentie-QTL.

De rol van diterpeenglycosiden en andere metabolieten in trips-resistentiemechanisme, gecodeerd door genen op in het QTL-gebied, is onderzocht. De relatieve aanwezigheid van metabolieten is bepaald in planten van de resistente accessie CGN16975, de vatbare accessie CGN17219, en in F4 planten met het resistentieallel of het vatbaarheidsallel homozygoot aanwezig in het QTL-gebied, voor twee verschillende behandelingen (wel of niet blootgesteld aan L1s). Zesendertig mogelijke diterpeenglycosiden die verschilden in relatieve aanwezigheid tussen de resistente en vatbare accessie zijn gedetecteerd. Achttien van deze mogelijke diterpeenglycosiden waren meer aanwezig in de resistente accessie, en de andere achttien waren meer aanwezig in de vatbare accessie. $\mathrm{Er}$ zijn geen verschillen in relatieve aanwezigheid van mogelijke metabolieten aangetoond tussen de verschillende groepen F4 planten voor beide behandelingen. Uit deze analyse kan worden geconcludeerd dat diterpeen- 
glycosiden geen rol spelen in het resistentiemechanisme dat wordt gecodeerd door genen in het resistentie-QTL op chromosoom 6.

Het effect van het QTL op chromosoom 6 op de resistentie tegen twee tripssoorten, namelijk Frankliniella occidentalis en tabakstrips, Thrips tabaci, wanneer ingekruist in vier verschillende vatbare Capsicum-achtergronden is bepaald. Het onderbreken van de ontwikkeling van de larven van het L1 naar het L2 stadium is vergeleken tussen planten met het resistentieallel homozygoot aanwezig in het QTL-gebied, en planten met het vatbaarheidsallel homozygoot aanwezig, binnen dezelfde genetische achtergrond. Ondanks dat er verschillende niveaus van resistentie zijn waargenomen tussen de verschillende genetische achtergronden, wat erop wijst dat er andere genetische factoren zijn in de verschillende achtergronden die trips-resistentie beïnvloeden, heb ik aangetoond dat het QTL in alle geteste achtergronden de larvale ontwikkeling van beide tripssoorten beïnvloedt. Deze bevinding laat zien dat het QTL kan worden gebruikt in de veredeling van tripsresistente Capsicum-rassen.

De bijdrage van elk van de twee trips-resistentie-QTLs, of een combinatie van beide QTLs, aan trips-resistentie is getoetst in een geen-keuze experiment. Een intermediair trips-resistentieniveau is waargenomen in planten van de nearisogenic line (NIL), een lijn die alleen voor het QTL-gebied op chromosoom 5 het resistentieallel homozygoot aanwezig heeft, die is toegevoegd bij de patentaanvraag waarin dit QTL is beschreven. Het QTL op chromosoom 5 had geen effect op trips-resistentie in twee verschillende experimenten, waarin de ontwikkeling van de larven en de zilverachtige schade op de hele plant werden bepaald. $\mathrm{Er}$ is in deze experimenten wel een significant effect van het QTL op chromosoom 6 aangetoond. Daarom veronderstel ik dat de bij de patentaanvraag toegevoegde NIL het resistentie-allel van chromosoom 6 heeft, dat afkomstig is van CGN16975. Deze bevindingen laten zien dat het QTL op chromosoom 6 onafhankelijk werkt van het QTL op chromosoom 5 in alle geteste genetische achtergronden.

In de algemene discussie bespreek ik de mogelijke manieren van aanpak om het causale gen (of de causale genen) dat ervoor zorgt dat de plant weerbaar wordt tegen trips, te identificeren, en de toekomstige richtingen van de veredeling van trips-resistentie in peper. Deze thesis draagt bij aan het beschermen van peper en paprika tegen trips: ten eerste, door meer inzicht te geven in het effect van plant- en bladleeftijd op trips-resistentie, wat bijdraagt aan het verbeteren van geïntegreerde gewasbescherming; ten tweede, door het fijnkarteren van het resistentie-QTL tot een gebied van $0.4 \mathrm{Mbp}$, wat kan worden ingekruist door veredelaars met een beperkt risico op het meekruisen van ongewenste eigenschappen; ten derde, door de selectie van kandidaatgenen in het resistentie- 
QTL, en dus door een goed uitgangspunt te creëren om het causale gen te identificeren, wat weer kan bijdragen aan het overbrengen van dit resistentiemechanisme naar andere vatbare Solanaceae; en ten slotte, door het effect van het QTL in verschillende genetische achtergronden op twee tripssoorten aan te tonen, en daarmee te laten zien dat dit QTL kan worden gebruikt als resistentiebron voor het veredelen van tripsresistente Capsicum-rassen. 


\section{References}

Abe, H., T. Shimoda, J. Ohnishi, S. Kugimiya, M. Narusaka, S. Seo, Y. Narusaka, S. Tsuda, and M. Kobayashi, 2009: Jasmonate-dependent plant defense restricts thrips performance and preference. BMC Plant Biology 9, 97.

Agrawal, S., N. Chandra, and S.L. Kothari, 1989: Plant regeneration in tissue cultures of pepper (Capsicum annuum L. cv. mathania). Plant Cell, Tissue and Organ Culture 16, 4755.

Alcantara, T.P., P.W. Bosland, and D.W. Smith, 1996: Ethyl methanesulfonate-induced seed mutagenesis of Capsicum annuum. Journal of Heredity 87, 239-241.

Alvarez, A.E., A.M. Alberti D'Amato, W.F. Tjallingii, M. Dicke, and B. Vosman, 2014: Response of Solanum tuberosum to Myzus persicae infestation at different stages of foliage maturity. Insect Science 21, 727-740.

Amin, P.W., 1979: Leaf curl disease of chilli peppers in Maharashtra, India. International Journal of Pest Management 25, 131-134.

Anaya-López, J.L., I. Torres-Pacheco, M. González-Chavira, J.A. Garzon-Tiznado, J.L. PonsHernandez, R.G. Guevara-González, C.I. Muñoz-Sánchez, L. Guevara-Olvera, R.F. RiveraBustamante, and S. Hernández-Verdugo, 2003: Resistance to geminivirus mixed infections in Mexican wild peppers. American Society for Horticultural Science 38, 251.

Anders, S., P.T. Pyl, and W. Huber, 2014: HTSeq-a Python framework to work with highthroughput sequencing data. Bioinformatics 31, 166-169.

Annadana, S., J. Peters, K. Gruden, A. Schipper, N.S. Outchkourov, M.J. Beekwilder, M. Udayakumar, and M.A. Jongsma, 2002: Effects of cysteine protease inhibitors on oviposition rate of the western flower thrips, Frankliniella occidentalis. Journal of Insect Physiology 48, 701-706.

Antignac, J.-P., K. de Wasch, F. Monteau, H. De Brabander, F. Andre, and B. Le Bizec, 2005: The ion suppression phenomenon in liquid chromatography-mass spectrometry and its consequences in the field of residue analysis. Analytica Chimica Acta 529, 129-136.

Arisha, M.H., S.N.M. Shah, Z.-H. Gong, H. Jing, C. Li, and H.-X. Zhang, 2015: Ethyl methane sulfonate induced mutations in M2 generation and physiological variations in M1 generation of peppers (Capsicum annuum L.). Frontiers in Plant Science 6.

Badenes-Perez, F.R., J. Gershenzon, and D.G. Heckel, 2014: Insect attraction versus plant defense: young leaves high in glucosinolates stimulate oviposition by a specialist herbivore despite poor larval survival due to high saponin content. PLoS ONE 9, e95766.

Ballina-Gomez, H., E. Ruiz-Sanchez, W. Chan-Cupul, L. Latournerie-Moreno, L. HernándezAlvarado, I. Islas-Flores, and J.J. Zuñiga-Aguilar, 2013: Response of Bemisia tabaci Genn. (Hemiptera: Aleyrodidae) biotype B to genotypes of pepper Capsicum annuum (Solanales: Solanaceae). Neotropical Entomology 42, 205-210. 
Belliure, B., A. Janssen, P.C. Maris, D. Peters, and M.W. Sabelis, 2005: Herbivore arthropods benefit from vectoring plant viruses. Ecology Letters 8, 70-79.

Ben-Mahmoud, S., J.R. Smeda, T.M. Chappell, C. Stafford-Banks, C.H. Kaplinsky, T. Anderson, M.A. Mutschler, G.G. Kennedy, and D.E. Ullman, 2018: Acylsugar amount and fatty acid profile differentially suppress oviposition by western flower thrips, Frankliniella occidentalis, on tomato and interspecific hybrid flowers. PLoS ONE 13, e0201583.

Benjamini, Y., and Y. Hochberg, 1995: Controlling the false discovery rate: a practical and powerful approach to multiple testing. Journal of the Royal Statistical Society: Series B (Methodological) 57, 289-300.

Berardini, T.Z., L. Reiser, D. Li, Y. Mezheritsky, R. Muller, E. Strait, and E. Huala, 2015: The arabidopsis information resource: making and mining the "gold standard" annotated reference plant genome. Genesis 53, 474-485.

Berger, S., T. Mitchell-Olds, and H.U. Stotz, 2002: Local and differential control of vegetative storage protein expression in response to herbivore damage in Arabidopsis thaliana. Physiologia Plantarum 114, 85-91.

Berger, S., E. Bell, A. Sadka, and J.E. Mullet, 1995: Arabidopsis thaliana Atvsp is homologous to soybean VspA and VspB, genes encoding vegetative storage protein acid phosphatases, and is regulated similarly by methyl jasmonate, wounding, sugars, light and phosphate. Plant Molecular Biology 27, 933-942.

Bethesda (MD), 2012: NCBI Genome Data Viewer, Vol. 2019. National Library of Medicine (US), National Center for Biotechnology Information.

Bielza, P., 2008: Insecticide resistance management strategies against the western flower thrips, Frankliniella occidentalis. Pest Management Science 64, 1131-1138.

Boege, K., and R.J. Marquis, 2005: Facing herbivory as you grow up: the ontogeny of resistance in plants. Trends in Ecology \& Evolution 20, 441-448.

Bohlmann, J., G. Meyer-Gauen, and R. Croteau, 1998: Plant terpenoid synthases: molecular biology and phylogenetic analysis. Proceedings of the National Academy of Sciences 95, 4126-4133.

Boiteux, L.S., and A.C. de Ávila, 1994: Inheritance of a resistance specific to tomato spotted wilt tospovirus in Capsicum chinense 'PI 159236'. Euphytica 75, 139-142.

Boiteux, L.S., T. Nagata, W.P. Dutra, and M.E.N. Fonseca, 1993: Sources of resistance to tomato spotted wilt virus (TSWV) in cultivated and wild species of Capsicum. Euphytica 67, 89-94.

Bos, J.I.B., D. Prince, M. Pitino, M.E. Maffei, J. Win, and S.A. Hogenhout, 2010: A functional genomics approach identifies candidate effectors from the aphid species Myzus persicae (green peach aphid). PLoS Genetics 6, e1001216.

Bosland, P.W., and J.J. Ellington, 1996: Comparison of Capsicum annuum and C. pubescens for antixenosis as a means of aphid resistance. HortScience 31, 1017. 
Brødsgaard, H.F., 1994: Effect of photoperiod on the bionomics of Frankliniella occidentalis (Pergande) (Thysanoptera, Thripidae). Journal of Applied Entomology 117, 498-507.

Broekgaarden, C., T.A.L. Snoeren, M. Dicke, and B. Vosman, 2011: Exploiting natural variation to identify insect-resistance genes. Plant Biotechnology Journal 9, 819-825.

Broekgaarden, C., P. Riviere, G. Steenhuis, M. Del sol Cuenca, M. Kos, and B. Vosman, 2012: Phloem-specific resistance in Brassica oleracea against the whitefly Aleyrodes proletella. Entomologia Experimentalis et Applicata 142, 153-164.

Broekgaarden, C., K.T.B. Pelgrom, J. Bucher, N.M. van Dam, K. Grosser, C.M.J. Pieterse, M. van Kaauwen, G. Steenhuis, R.E. Voorrips, M. de Vos, B. Vosman, A. Worrich, and S.C.M. van Wees, 2018: Combining QTL mapping with transcriptome and metabolome profiling reveals a possible role for $A B A$ signaling in resistance against the cabbage whitefly in cabbage. PLoS ONE 13, e0206103.

Broughton, S., and G.A. Herron, 2009: Potential new insecticides for the control of western flower thrips (Thysanoptera: Thripidae) on sweet pepper, tomato, and lettuce. Journal of Economic Entomology 102, 646-651.

Calvo, F.J., K. Bolckmans, and J.E. Belda, 2012: Biological control-based IPM in sweet pepper greenhouses using Amblyseius swirskii (Acari: Phytoseiidae). Biocontrol Science and Technology 22, 1398-1416.

Camacho, D., A. de la Fuente, and P. Mendes, 2005: The origin of correlations in metabolomics data. Metabolomics 1, 53-63.

Cannon, R.J.C., L. Matthews, and D.W. Collins, 2007: A review of the pest status and control options for Thrips palmi. Crop Protection 26, 1089-1098.

Capinera, J., 2001: Handbook of vegetable pests Elsevier, San Diego, California.

Carolan, J.C., D. Caragea, K.T. Reardon, N.S. Mutti, N. Dittmer, K. Pappan, F. Cui, M. Castaneto, J. Poulain, C. Dossat, D. Tagu, J.C. Reese, G.R. Reeck, T.L. Wilkinson, and O.R. Edwards, 2011: Predicted effector molecules in the salivary secretome of the pea aphid (Acyrthosiphon pisum): a dual transcriptomic/proteomic approach. Journal of Proteome Research 10, 1505-1518.

Chen, F., D. Tholl, J. Bohlmann, and E. Pichersky, 2011: The family of terpene synthases in plants: a mid-size family of genes for specialized metabolism that is highly diversified throughout the kingdom. The Plant Journal 66, 212-229.

Chen, R., H. Li, L. Zhang, J. Zhang, J. Xiao, and Z. Ye, 2007: CaMi, a root-knot nematode resistance gene from hot pepper (Capsium annuum L.) confers nematode resistance in tomato. Plant Cell Reports 26, 895-905.

Chen, Y.H., R. Gols, and B. Benrey, 2015: Crop domestication and its impact on naturally selected trophic interactions. Annual Review of Entomology 60, 35-58.

Cheng, L., Y. Wang, L. Meng, X. Hu, Y. Cui, Y. Sun, L. Zhu, J. Ali, J. Xu, and Z. Li, 2011: Identification of salt-tolerant QTLs with strong genetic background effect using two sets of reciprocal introgression lines in rice. Genome 55, 45-55. 
Chisholm, I.F., and T. Lewis, 1984: A new look at thrips (Thysanoptera) mouthparts, their action and effects of feeding on plant tissue. Bulletin of Entomological Research 74, 663675.

Choi, Y., G.E. Sims, S. Murphy, J.R. Miller, and A.P. Chan, 2012: Predicting the functional effect of amino acid substitutions and indels. PLoS ONE 7, e46688.

Christ, B., I. Süssenbacher, S. Moser, N. Bichsel, A. Egert, T. Müller, B. Kräutler, and S. Hörtensteiner, 2013: Cytochrome P450 CYP89A9 is involved in the formation of major chlorophyll catabolites during leaf senescence in arabidopsis. The Plant Cell 25, 1868-1880.

Christoffersen Rolf, E., W. Percival Frank, and R. Bozak Kristin, 1995: Functional and DNA sequence divergence of the CYP71 gene family in higher plants. Drug Metabolism and Drug Interactions 12, 207.

Cloyd, R.A., 2009: Western flower thrips (Frankliniella occidentalis) management on ornamental crops grown in greenhouses: have we reached an impasse. Pest Technology 3, $1-9$.

Collu, G., N. Unver, A.M.G. Peltenburg-Looman, R. van der Heijden, R. Verpoorte, and J. Memelink, 2001: Geraniol 10-hydroxylase11, a cytochrome P450 enzyme involved in terpenoid indole alkaloid biosynthesis. FEBS Letters 508, 215-220.

Cooper, W.R., J.W. Dillwith, and G.J. Puterka, 2011: Comparisons of salivary proteins from five aphid (Hemiptera: Aphididae) species. Environmental Entomology 40, 151-156.

Daryanto, A., M. Syukur, P. Hidayat, and A. Maharijaya, 2016: Antixenosis and antibiosis based resistance of chilli pepper to melon aphid. Journal of Applied Horticulture 19, 147151.

De Kogel, W.J., M. van der Hoek, and C. Mollema, 1997a: Variation in performance of western flower thrips populations on susceptible and partially resistant cucumber. Entomologia Experimentalis et Applicata 83, 73-80.

De Kogel, W.J., A. Balkema-Boomstra, M. Van der Hoek, S. Zijlstra, and C. Mollema, 1997b: Resistance to western flower thrips in greenhouse cucumber: effect of leaf position and plant age on thrips reproduction. Euphytica 94, 63-67.

De Lange, E.S., D. Balmer, B. Mauch-Mani, and T.C.J. Turlings, 2014: Insect and pathogen attack and resistance in maize and its wild ancestors, the teosintes. New Phytologist 204, 329-341.

Diamond, J., 2002: Evolution, consequences and future of plant and animal domestication. Nature 418, 700-707.

Díaz, I., R. Moreno, and J.B. Power, 1988: Plant regeneration from protoplasts of Capsicum annuum. Plant Cell Reports 7, 210-212.

Ding, T., H. Chi, A. Gökçe, Y. Gao, and B. Zhang, 2018: Demographic analysis of arrhenotokous parthenogenesis and bisexual reproduction of Frankliniella occidentalis (Pergande) (Thysanoptera: Thripidae). Scientific Reports 8, 3346. 
Dinh, S.T., I. Gális, and I.T. Baldwin, 2013: UVB radiation and 17-hydroxygeranyllinalool diterpene glycosides provide durable resistance against mirid (Tupiocoris notatus) attack in field-grown Nicotiana attenuata plants. Plant, Cell \& Environment 36, 590-606.

Dobin, A., C.A. Davis, F. Schlesinger, J. Drenkow, C. Zaleski, S. Jha, P. Batut, M. Chaisson, and T.R. Gingeras, 2012: STAR: ultrafast universal RNA-seq aligner. Bioinformatics 29, 1521.

Doebley, J.F., B.S. Gaut, and B.D. Smith, 2006: The molecular genetics of crop domestication. Cell 127, 1309-1321.

Eapen, S., 2011: Pollen grains as a target for introduction of foreign genes into plants: an assessment. Physiology and molecular biology of plants : an international journal of functional plant biology 17, 1-8.

Eigenbrode, S.D., and K.E. Espelie, 1995: Effects of plant epicuticular lipids on insect herbivores. Annual review of entomology 40, 171-194.

Eigenbrode, S.D., H. Ding, P. Shiel, and P.H. Berger, 2002: Volatiles from potato plants infected with potato leafroll virus attract and arrest the virus vector, Myzus persicae (Homoptera: Aphididae). Proceedings of the Royal Society of London. Series B: Biological Sciences 269, 455-460.

Elzinga, D.A., M. De Vos, and G. Jander, 2014: Suppression of plant defenses by a Myzus persicae (green peach aphid) salivary effector protein. Molecular Plant-Microbe Interactions 27, 747-756.

Fery, R.L., and J.M. Schalk, 1991: Resistance in pepper (Capsicum annuum L.) to western flower thrips [Frankliniella occidentalis (Pergande)]. HortScience 26, 1073.

Fiehn, O., 2002: Metabolomics - the link between genotypes and phenotypes, In: C. Town, (ed.) Functional Genomics, 155-171. Springer Netherlands, Dordrecht.

Firdaus, S., A. Van Heusden, A. Harpenas, E.D.J. Supena, R.G.F. Visser, and B. Vosman, 2011: Identification of silverleaf whitefly resistance in pepper. Plant Breeding 130, 708-714.

Fraenkel, G., 1953: Studies on the distribution of vitamin BT (carnitine). The Biological Bulletin 104, 359-371.

Frantz, J.D., J. Gardner, M.P. Hoffmann, and M.M. Jahn, 2004: Greenhouse screening of Capsicum accessions for resistance to green peach aphid (Myzus persicae). HortScience 39, 1332-1335.

Frei, A., C. Cardona, H. Gu, J.M. Bueno, and S. Dorn, 2003: Antixenosis and antibiosis of common beans to Thrips palmi Karny (Thysanoptera: Thripidae). Journal of Economic Entomology 96, 1577-1584.

Frei, A., M.W. Blair, C. Cardona, S.E. Beebe, H. Gu, and S. Dorn, 2005: QTL mapping of resistance to Thrips palmi Karny in common bean. Crop Science 45, 379-387.

Friedli, J., and S. Bacher, 2001: Mutualistic interaction between a weevil and a rust fungus, two parasites of the weed Cirsium arvense. Oecologia 129, 571-576. 
Fulton, T.M., J. Chunwongse, and S.D. Tanksley, 1995: Microprep protocol for extraction of DNA from tomato and other herbaceous plants. Plant Molecular Biology Reporter 13, 207209.

Gasteiger, E., A. Gattiker, C. Hoogland, I. Ivanyi, R.D. Appel, and A. Bairoch, 2003: ExPASy: the proteomics server for in-depth protein knowledge and analysis. Nucleic Acids Research 31, 3784-3788.

Gent, D.H., L.J. du Toit, S.F. Fichtner, S.K. Mohan, H.R. Pappu, and H.F. Schwartz, 2006: Iris yellow spot virus: an emerging threat to onion bulb and seed production. Plant Disease 90, 1468-1480.

German, T.L., D.E. Ullman, and J.W. Moyer, 1992: Tospoviruses: diagnosis, molecular biology, phylogeny, and vector relationships. Annual Review of Phytopathology 30, 315348.

Glas, J., B. Schimmel, J. Alba, R. Escobar-Bravo, R. Schuurink, and M. Kant, 2012: Plant glandular trichomes as targets for breeding or engineering of resistance to herbivores. International Journal of Molecular Sciences 13, 17077.

Gols, R., T. Bukovinszky, N.M. van Dam, M. Dicke, J.M. Bullock, and J.A. Harvey, 2008: Performance of generalist and specialist herbivores and their endoparasitoids differs on cultivated and wild Brassica populations. Journal of Chemical Ecology 34, 132-143.

Gonçalves, L.S., V.M. Gomes, R.R. Robaina, R.H. Valim, R. Rodrigues, and F.M. Aranha, 2014: Resistance to root-knot nematode (Meloidogyne enterolobii) in Capsicum spp. accessions. Revista Brasileira de Ciências Agrárias 9, 49-52.

Grube, R.C., Y. Zhang, J.F. Murphy, F. Loaiza-Figueroa, V.K. Lackney, R. Provvidenti, and M.K. Jahn, 2000: New source of resistance to cucumber mosaic virus in Capsicum frutescens. Plant Disease 84, 885-891.

Gunay, A.L., and P.S. Rao, 1978: In vitro plant regeneration from hypocotyl and cotyledon explants of red pepper (Capsicum). Plant Science Letters 11, 365-372.

Hansen, E.A., J.E. Funderburk, S.R. Reitz, S. Ramachandran, J.E. Eger, and H. McAuslane, 2003: Within-plant distribution of Frankliniella species (Thysanoptera: Thripidae) and Orius insidiosus (Heteroptera: Anthocoridae) in field pepper. Environmental Entomology 32, 1035-1044.

Harmel, N., E. Létocart, A. Cherqui, P. Giordanengo, G. Mazzucchelli, F. Guillonneau, E. De Pauw, E. Haubruge, and F. Francis, 2008: Identification of aphid salivary proteins: a proteomic investigation of Myzus persicae. Insect Molecular Biology 17, 165-174.

Heiling, S., S. Khanal, A. Barsch, G. Zurek, I.T. Baldwin, and E. Gaquerel, 2016: Using the knowns to discover the unknowns: MS-based dereplication uncovers structural diversity in 17-hydroxygeranyllinalool diterpene glycoside production in the Solanaceae. The Plant Journal 85, 561-577.

Heiling, S., M.C. Schuman, M. Schoettner, P. Mukerjee, B. Berger, B. Schneider, A.R. Jassbi, and I.T. Baldwin, 2010: Jasmonate and ppHsystemin regulate key malonylation steps in the 
biosynthesis of 17-hydroxygeranyllinalool diterpene glycosides, an abundant and effective direct defense against herbivores in Nicotiana attenuata. The Plant Cell 22, 273-292.

Heitor Valim, R., L. Simoes Azeredo Goncalves, V. Martins Gomes, R. Rodrigues Robaina, C. Dos Santos Bento, and R. Rodrigues, 2013: Resistance to root-knot nematode (Meloidogyne enterolobii) in Capsicum spp. accessions Breakthroughs in the genetics and breeding of capsicum and eggplant; Proceedings of the XV EUCARPIA meeting. Comitato per I'organizzazione degli eventi (COE) DISAFA, Università degli ....

Herms, D.A., and W.J. Mattson, 1992: The dilemma of plants: to grow or defend. The Quarterly Review of Biology 67, 283-335.

Hernández-Verdugo, S., R.G. Guevara-González, R.F. Rivera-Bustamante, and K. Oyama, 2001: Screening wild plants of Capsicum annuum for resistance to pepper huasteco virus (PHV): presence of viral DNA and differentiation among populations. Euphytica 122, 31-36.

Hidayat, S.H., and E. Rahmayani, 2007: Transmission of tomato leaf curl begomovirus by two different species of whitefly (Hemiptera: Aleyrodidae). The Plant Pathology Journal 23, 57-61.

Höfer, R., L. Dong, F. André, J.-F. Ginglinger, R. Lugan, C. Gavira, S. Grec, G. Lang, J. Memelink, S. Van Der Krol, H. Bouwmeester, and D. Werck-Reichhart, 2013: Geraniol hydroxylase and hydroxygeraniol oxidase activities of the CYP76 family of cytochrome P450 enzymes and potential for engineering the early steps of the (seco)iridoid pathway. Metabolic Engineering 20, 221-232.

Hogenhout, S.A., and J.I.B. Bos, 2011: Effector proteins that modulate plant-insect interactions. Current Opinion in Plant Biology 14, 422-428.

Hulse-Kemp, A.M., S. Maheshwari, K. Stoffel, T.A. Hill, D. Jaffe, S.R. Williams, N. Weisenfeld, S. Ramakrishnan, V. Kumar, P. Shah, M.C. Schatz, D.M. Church, and A. Van Deynze, 2018: Reference quality assembly of the 3.5-Gb genome of Capsicum annuum from a single linkedread library. Horticulture Research 5, 4.

Hulshof, J., and I. Vanninen, 2002: Western flower thrips feeding on pollen, and its implications for control Thrips and tospoviruses: proceedings of the 7 th international symposium on Thysanoptera, 173-179, Vol. 7.

IBM Corp., 2015: IBM SPSS Statistics for Windows 23.0 ed, Armonk, NY.

Ishida, H., T. Murai, S. Sonoda, H. Yoshida, Y. Izumi, and H. Tsumuki, 2003: Effects of temperature and photoperiod on development and oviposition of Frankliniella occidentalis (Pergande)(Thysanoptera: Thripidae). Applied entomology and zoology 38, 65-68.

Jahn, M., I. Paran, K. Hoffmann, E.R. Radwanski, K.D. Livingstone, R.C. Grube, E. Aftergoot, M. Lapidot, and J. Moyer, 2000: Genetic mapping of the Tsw locus for resistance to the tospovirus tomato spotted wilt virus in Capsicum spp. and its relationship to the $S w-5$ gene for resistance to the same pathogen in tomato. Molecular Plant-Microbe Interactions 13, 673-682. 
Jassbi, A.R., S. Zamanizadehnajari, D. Kessler, and I.T. Baldwin, 2006: A new acyclic diterpene glycoside from Nicotiana attenuata with a mild deterrent effect on feeding Manduca sexta larvae. Zeitschrift für Naturforschung B 61, 1138.

Jensen, S.E., 2000: Insecticide resistance in the western flower thrips, Frankliniella occidentalis. Integrated Pest Management Reviews 5, 131-146.

Jenser, G., and Á. Szénási, 2004: Review of the biology and vector capability of Thrips tabaci Lindeman (Thysanoptera: Thripidae). Acta Phytopathologica et Entomologica Hungarica 39, 137-155.

Johari, A., S. Herlinda, Y. Pujiastuti, C. Irsan, and D. Sartiami, 2014: Morphological and genetic variation of Thrips parvispinus (Thysanoptera: Thripidae) in chili plantation (Capsicum annuum L.) in the lowland and highland of Jambi province, Indonesia. American Journal of BioScience 2, 17-21.

Johnson, S.N., A.E. Douglas, S. Woodward, and S.E. Hartley, 2003: Microbial impacts on plant-herbivore interactions: the indirect effects of a birch pathogen on a birch aphid. Oecologia 134, 388-396.

Jones, D.R., 2005: Plant viruses transmitted by thrips. European Journal of Plant Pathology 113, 119-157.

Kahn, R.A., R.L. Bouquin, F. Pinot, I. Benveniste, and F. Durst, 2001: A conservative amino acid substitution alters the regiospecificity of CYP94A2, a fatty acid hydroxylase from the plant Vicia sativa. Archives of Biochemistry and Biophysics 391, 180-187.

Karban, R., 1989: Fine-scale adaptation of herbivorous thrips to individual host plants. Nature 340, 60-61.

Keinänen, M., N.J. Oldham, and I.T. Baldwin, 2001: Rapid HPLC screening of jasmonateinduced increases in tobacco alkaloids, phenolics, and diterpene glycosides in Nicotiana attenuata. Journal of Agricultural and Food Chemistry 49, 3553-3558.

Kenyon, L., S. Kumar, W.-S. Tsai, J.d.A. Hughes, G. Loebenstein, and N. Katis, 2014: Chapter six - Virus diseases of peppers (Capsicum spp.) and their control. Advances in Virus Research 90, 297-354.

Kimble, K.A., and R.G. Grogan, 1960: Resistance to phytophthora root rot in pepper. Phytopathology 50, $642 \mathrm{p}$.

Kindt, F., N.N. Joosten, D. Peters, and W.F. Tjallingii, 2003: Characterisation of the feeding behaviour of western flower thrips in terms of electrical penetration graph (EPG) waveforms. Journal of Insect Physiology 49, 183-191.

Kirk, W.D., 2002: The pest and vector from the West: Frankliniella occidentalis Thrips and Tospoviruses: Proceedings of the 7th international symposium on thysanoptera, 33-42, Vol. 2. Australian National Insect Collection Canberra, Australia.

Kirk, W.D.J., 1985: Pollen-feeding and the host specificity and fecundity of flower thrips (Thysanoptera). Ecological Entomology 10, 281-289. 
Kirk, W.D.J., and L.I. Terry, 2003: The spread of the western flower thrips Frankliniella occidentalis (Pergande). Agricultural and Forest Entomology 5, 301-310.

Klein, T.M., R. Arentzen, P.A. Lewis, and S. Fitzpatrick-McElligott, 1992: Transformation of microbes, plants and animals by particle bombardment. Bio/Technology 10, 286-291.

Koch, K.G., K. Chapman, J. Louis, T. Heng-Moss, and G. Sarath, 2016: Plant tolerance: a unique approach to control hemipteran pests. Frontiers in Plant Science $\mathbf{7}$.

Kogan, M., and E.F. Ortman, 1978: Antixenosis-a new term proposed to define Painter's "Nonpreference" modality of resistance. Bulletin of the Entomological Society of America 24, 175-176.

Koschier, E.H., W.J. De Kogel, and J.H. Visser, 2000: Assessing the attractiveness of volatile plant compounds to western flower thrips Frankliniella occidentalis. Journal of Chemical Ecology 26, 2643-2655.

Krishna Kumar, N.K., D.E. Ullman, and J.J. Cho, 1995: Resistance among Lycopersicon species to Frankliniella occidentalis (Thysanoptera: Thripidae). Journal of Economic Entomology 88, 1057-1065.

Krishna Kumar, N.K., M. Aradya, A.A. Deshpande, N. Anand, and P.R. Ramachandar, 1996: Initial screening of chili and sweet pepper germplasm for resistance to chili thrips, Scirtothrips dorsalis Hood. Euphytica 89, 319-324.

Kritzman, A., M. Lampel, B. Raccah, and A. Gera, 2001: Distribution and transmission of iris yellow spot virus. Plant Disease 85, 838-842.

Küfner, I., and W. Koch, 2008: Stress regulated members of the plant organic cation transporter family are localized to the vacuolar membrane. BMC Research Notes 1, 43.

Kumar, R.V., V.K. Sharma, B. Chattopadhyay, and S. Chakraborty, 2012: An improved plant regeneration and Agrobacterium - mediated transformation of red pepper (Capsicum annuum L.). Physiology and Molecular Biology of Plants 18, 357-364.

Kumar, S., S. Kumar, M. Singh, A.K. Singh, and M. Rai, 2006: Identification of host plant resistance to pepper leaf curl virus in chilli (Capsicum species). Scientia Horticulturae 110, 359-361.

Lange, B.M., T. Rujan, W. Martin, and R. Croteau, 2000: Isoprenoid biosynthesis: the evolution of two ancient and distinct pathways across genomes. Proceedings of the National Academy of Sciences 97, 13172-13177.

Langmead, B., and S.L. Salzberg, 2012: Fast gapped-read alignment with Bowtie 2. Nature Methods 9, 357.

Láska, P., J. Betlach, and M. Havránková, 1982: Resistance to the glasshouse whitefly (Trialeurodes vaporariorum Westw.) in sweet pepper (Capsicum annuum L.). Euphytica 31, 977-980.

Latournerie-Moreno, L., A. Ic-Caamal, E. Ruiz-Sánchez, H. Ballina-Gómez, I. Islas-Flores, W. Chan-Cupul, and D. González-Mendoza, 2015: Survival of Bemisia tabaci and activity of 
plant defense-related enzymes in genotypes of Capsicum annuum L. Chilean journal of agricultural research $75,71-77$.

Lebeau, A., M.-C. Daunay, A. Frary, A. Palloix, J.-F. Wang, J. Dintinger, F. Chiroleu, E. Wicker, and P. Prior, 2011: Bacterial wilt resistance in tomato, pepper, and eggplant: genetic resources respond to diverse strains in the Ralstonia solanacearum species complex. Phytopathology 101, 154-165.

Lee, H.-R., I.-H. Bae, S.-W. Park, H.-J. Kim, W.-K. Min, J.-H. Han, K.-T. Kim, and B.-D. Kim, 2009: Construction of an integrated pepper map using RFLP, SSR, CAPS, AFLP, WRKY, rRAMP, and BAC end sequences. Molecules and Cells 27, 21-37.

Leiss, K.A., Y.H. Choi, I.B. Abdel-Farid, R. Verpoorte, and P.G.L. Klinkhamer, 2009a: NMR Metabolomics of thrips (Frankliniella occidentalis) resistance in Senecio hybrids. Journal of Chemical Ecology 35, 219-229.

Leiss, K.A., F. Maltese, Y.H. Choi, R. Verpoorte, and P.G.L. Klinkhamer, 2009b: Identification of chlorogenic acid as a resistance factor for thrips in chrysanthemum. Plant Physiology 150, 1567-1575.

Leiss, K.A., G. Cristofori, R. van Steenis, R. Verpoorte, and P.G.L. Klinkhamer, 2013: An eco-metabolomic study of host plant resistance to Western flower thrips in cultivated, biofortified and wild carrots. Phytochemistry 93, 63-70.

Leite, G.L.D., M. Picanço, R.N.C. Guedes, and J.C. Zanuncio, 2001: Role of plant age in the resistance of Lycopersicon hirsutum f. glabratum to the tomato leafminer Tuta absoluta (Lepidoptera: Gelechiidae). Scientia Horticulturae 89, 103-113.

Lelandais-Brière, C., M. Jovanovic, G.A.M. Torres, Y. Perrin, R. Lemoine, F. Corre-Menguy, and C. Hartmann, 2007: Disruption of AtOCT1, an organic cation transporter gene, affects root development and carnitine-related responses in Arabidopsis. The Plant Journal 51, 154164.

Lewis, T., 1973: Thrips, their biology, ecology and economic importance, $x v+349 \mathrm{pp}$. Academic Press, London.

Li, H., 2013: Aligning sequence reads, clone sequences and assembly contigs with BWAMEM. arXiv:1303.3997.

Liao, C.Y., P. Wu, B. Hu, and K.K. Yi, 2001: Effects of genetic background and environment on QTLs and epistasis for rice (Oryza sativa L.) panicle number. Theoretical and Applied Genetics 103, 104-111.

Liew, M., R. Pryor, R. Palais, C. Meadows, M. Erali, E. Lyon, and C. Wittwer, 2004: Genotyping of single-nucleotide polymorphisms by high-resolution melting of small amplicons. Clinical Chemistry 50, 1156-1164.

Linders, E.G.A., J.L.M.E. Nicolet, and H.J. Van Wijk, 2015: Insect resistant plant. Vol. PCT/EP2008/055374: Syngenta Participations AG Unites States. 
Liu, Y., J.-E. Ahn, S. Datta, R.A. Salzman, J. Moon, B. Huyghues-Despointes, B. Pittendrigh, L.L. Murdock, H. Koiwa, and K. Zhu-Salzman, 2005: Arabidopsis vegetative storage protein is an anti-insect acid phosphatase. Plant Physiology 139, 1545.

Lommen, A., 2009: MetAlign: Interface-driven, versatile metabolomics tool for hyphenated full-scan mass spectrometry data preprocessing. Analytical Chemistry 81, 3079-3086.

Long, R.M., and R. Croteau, 2005: Preliminary assessment of the C13-side chain 2'hydroxylase involved in taxol biosynthesis. Biochemical and Biophysical Research Communications 338, 410-417.

Lublinkhof, J., and D.E. Foster, 1977: Development and reproductive capacity of Frankliniella occidentalis (Thysanoptera: Thripidae) reared at three temperatures. Journal of the Kansas Entomological Society 50, 313-316.

Luo, R., B. Liu, Y. Xie, Z. Li, W. Huang, J. Yuan, G. He, Y. Chen, Q. Pan, Y. Liu, J. Tang, G. Wu, H. Zhang, Y. Shi, Y. Liu, C. Yu, B. Wang, Y. Lu, C. Han, D.W. Cheung, S.-M. Yiu, S. Peng, Z. Xiaoqian, G. Liu, X. Liao, Y. Li, H. Yang, J. Wang, T.-W. Lam, and J. Wang, 2012: SOAPdenovo2: an empirically improved memory-efficient short-read de novo assembler. GigaScience 1, 18.

Macel, M., I.G.S. Visschers, J.L. Peters, I.F. Kappers, R.C.H. de Vos, and N.M. van Dam, 2019: Metabolomics of thrips resistance in pepper (Capsicum spp.) reveals monomer and dimer acyclic diterpene glycosides as potential chemical defenses. Journal of Chemical Ecology 45, 490-501.

Maharijaya, A., 2013: Resistance to thrips in pepper. Doctoral thesis, Wageningen University, Wageningen.

Maharijaya, A., B. Vosman, K. Pelgrom, Y. Wahyuni, R.C.H. de Vos, and R.E. Voorrips, 2019: Genetic variation in phytochemicals in leaves of pepper (Capsicum) in relation to thrips resistance. Arthropod-Plant Interactions 13, 1-9.

Maharijaya, A., B. Vosman, G. Steenhuis-Broers, A. Harpenas, A. Purwito, R.G.F. Visser, and R.E. Voorrips, 2011: Screening of pepper accessions for resistance against two thrips species (Frankliniella occidentalis and Thrips parvispinus). Euphytica 177, 401-410.

Maharijaya, A., B. Vosman, G. Steenhuis-Broers, K. Pelgrom, A. Purwito, R.G.F. Visser, and R.E. Voorrips, 2015: QTL mapping of thrips resistance in pepper. Theoretical and Applied Genetics 128, 1945-1956.

Maharijaya, A., B. Vosman, F. Verstappen, G. Steenhuis-Broers, R. Mumm, A. Purwito, R.G. Visser, and R.E. Voorrips, 2012: Resistance factors in pepper inhibit larval development of thrips (Frankliniella occidentalis). Entomologia Experimentalis et Applicata 145, 62-71.

Mandal, B., H.R. Pappu, A.S. Csinos, and A.K. Culbreath, 2006: Response of peanut, pepper, tobacco, and tomato cultivars to two biologically distinct isolates of tomato spotted wilt virus. Plant Disease 90, 1150-1155.

Mandal, B., M.L. Wells, N. Martinez-Ochoa, A.S. Csinos, and H.R. Pappu, 2007: Symptom development and distribution of tomato spotted wilt virus in flue-cured tobacco. Annals of Applied Biology 151, 67-75. 
Maris, P.C., N.N. Joosten, D. Peters, and R.W. Goldbach, 2003: Thrips resistance in pepper and its consequences for the acquisition and inoculation of tomato spotted wilt virus by the western flower thrips. Phytopathology 93, 96-101.

Maris, P.C., N.N. Joosten, R.W. Goldbach, and D. Peters, 2004: Tomato spotted wilt virus infection improves host suitability for its vector Frankliniella occidentalis. Phytopathology 94, 706-711.

Mason, H.S., and J.E. Mullet, 1990: Expression of two soybean vegetative storage protein genes during development and in response to water deficit, wounding, and jasmonic acid. The Plant Cell 2, 569-579.

Matsunaga, H., and S. Monma, 1999: Sources of resistance to bacterial wilt in Capsicum. Journal of the Japanese Society for Horticultural Science 68, 753-761.

Mattson, W.J., 1980: Herbivory in relation to plant nitrogen content. Annual Review of Ecology and Systematics 11, 119-161.

McCall, A.C., and J.A. Fordyce, 2010: Can optimal defence theory be used to predict the distribution of plant chemical defences? Journal of Ecology 98, 985-992.

McConn, M., R.A. Creelman, E. Bell, J.E. Mullet, and J. Browse, 1997: Jasmonate is essential for insect defense in Arabidopsis. Proceedings of the National Academy of Sciences 94, 54735477.

McDonald, J.R., J.S. Bale, and K.F. Walters, 1998: Effect of temperature on development of the western flower thrips, Frankliniella occidentalis (Thysanoptera: Thripidae). European Journal of Entomology 95, 301-306.

Méndez-Vigo, B., J.M. Martínez-Zapater, and C. Alonso-Blanco, 2013: The flowering repressor SVP underlies a novel Arabidopsis thaliana QTL interacting with the genetic background. PLoS Genetics 9, e1003289.

Meyer, R.S., A.E. DuVal, and H.R. Jensen, 2012: Patterns and processes in crop domestication: an historical review and quantitative analysis of 203 global food crops. New Phytologist 196, 29-48.

Mirnezhad, M., R.R. Romero-González, K.A. Leiss, Y.H. Choi, R. Verpoorte, and P.G.L. Klinkhamer, 2010: Metabolomic analysis of host plant resistance to thrips in wild and cultivated tomatoes. Phytochemical Analysis 21, 110-117.

Mollema, C., M. Steenhuis, H. Inggamer, and C. Soria, 1993: Evaluating the resistance to Frankliniella occidentalis in cucumber: methods, genotypic variation and effects upon thrips biology. Bulletin OILB SROP (France).

Molyneux, D., and D. Jefferies, 1986: Feeding behaviour of pathogen-infected vectors. Parasitology 92, 721-736.

Morales, F.J., 2006: Tropical Whitefly IPM Project. Advances in Virus Research 69, 249-311.

Morales, F.J., and P.G. Jones, 2004: The ecology and epidemiology of whitefly-transmitted viruses in Latin America. Virus Research 100, 57-65. 
Moritz, G., S. Kumm, and L. Mound, 2004: Tospovirus transmission depends on thrips ontogeny. Virus Research 100, 143-149.

Morse, J.G., and M.S. Hoddle, 2006: Invasion biology of thrips. Annual Review of Entomology 51, 67-89.

Mouden, S., K.F. Sarmiento, P.G. Klinkhamer, and K.A. Leiss, 2017: Integrated pest management in western flower thrips: past, present and future. Pest Management Science 73, 813-822.

Mound, L.A., and A.K. Walker, 1982: Terebrantia (Insecta: Thysanoptera). Fauna of New Zealand $\mathbf{1}$.

Muchero, W., J.D. Ehlers, and P.A. Roberts, 2010: QTL analysis for resistance to foliar damage caused by Thrips tabaci and Frankliniella schultzei (Thysanoptera: Thripidae) feeding in cowpea [Vigna unguiculata (L.) Walp.]. Molecular Breeding 25, 47-56.

Mugford, S.T., E. Barclay, C. Drurey, K.C. Findlay, and S.A. Hogenhout, 2016: An immunosuppressive aphid saliva protein is delivered into the cytosol of plant mesophyll cells during feeding. Molecular Plant-Microbe Interactions 29, 854-861.

Mumford, R.A., I. Barker, and K.R. Wood, 1996: The biology of the tospoviruses. Annals of Applied Biology 128, 159-183.

Murai, T., 2000: Effect of temperature on development and reproduction of the onion thrips, Thrips tabaci Lindeman (Thysanoptera: Thripidae), on pollen and honey solution. Applied Entomology and Zoology 35, 499-504.

Ng, J.C.K., and K.L. Perry, 2004: Transmission of plant viruses by aphid vectors. Molecular Plant Pathology 5, 505-511.

Nijveen, H., M. van Kaauwen, D.G. Esselink, B. Hoegen, and B. Vosman, 2013: QualitySNPng: a user-friendly SNP detection and visualization tool. Nucleic Acids Research 41, W587-W590.

Nuessly, G.S., and R.T. Nagata, 1995: Pepper varietal response to thrips feeding, In: B. L. Parker, M. Skinner and T. Lewis, (eds.) Thrips Biology and Management, 115-118. Springer US, Boston, MA.

Ohnishi, T., T. Nomura, B. Watanabe, D. Ohta, T. Yokota, H. Miyagawa, K. Sakata, and M. Mizutani, 2006: Tomato cytochrome P450 CYP734A7 functions in brassinosteroid catabolism. Phytochemistry 67, 1895-1906.

Omo-Ikerodah, E.E., I. Fawole and C. A. Fatokun, 2008: Genetic mapping of quantitative trait loci (QTLs) with effects on resistance to flower bud thrips (Megalurothrips sjostedti) identified in recombinant inbred lines of cowpea (Vigna unguiculata (L.) Walp). African Journal of Biotechnology 7, 263-270.

Outchkourov, N.S., W.J. De Kogel, A. Schuurman-de Bruin, M. Abrahamson, and M.A. Jongsma, 2004: Specific cysteine protease inhibitors act as deterrents of western flower thrips, Frankliniella occidentalis (Pergande), in transgenic potato. Plant Biotechnology Journal 2, 439-448. 
Painter, R., 1951: The mechanisms of resistance Insect resistance in crop plants, 23-83. Macmillan, New York.

Park, H.-H., J.-H. Lee, and K.-B. Uhm, 2007: Economic thresholds of western flower thrips (Thysanoptera: Thripidae) for unripe red pepper in greenhouse. Journal of Asia-Pacific Entomology 10, 45-53.

Peddaboina, V., C. Thamidala, and S. Karampuri, 2006: In vitro shoot multiplication and plant regeneration in four Capsicum species using thidiazuron. Scientia Horticulturae 107, 117-122.

Peter, K., R. Goth, and R. Webb, 1984: Indian hot peppers as new sources of resistance to bacterial wilt, Phytophthora root rot, and root-knot nematode. HortScience 19, 277-278.

Qin, C., C. Yu, Y. Shen, X. Fang, L. Chen, J. Min, J. Cheng, S. Zhao, M. Xu, Y. Luo, Y. Yang, Z. Wu, L. Mao, H. Wu, C. Ling-Hu, H. Zhou, H. Lin, S. González-Morales, D.L. TrejoSaavedra, H. Tian, X. Tang, M. Zhao, Z. Huang, A. Zhou, X. Yao, J. Cui, W. Li, Z. Chen, Y. Feng, Y. Niu, S. Bi, X. Yang, W. Li, H. Cai, X. Luo, S. Montes-Hernández, M.A. LeyvaGonzález, Z. Xiong, X. He, L. Bai, S. Tan, X. Tang, D. Liu, J. Liu, S. Zhang, M. Chen, L. Zhang, L. Zhang, Y. Zhang, W. Liao, Y. Zhang, M. Wang, X. Lv, B. Wen, H. Liu, H. Luan, Y. Zhang, S. Yang, X. Wang, J. Xu, X. Li, S. Li, J. Wang, A. Palloix, P.W. Bosland, Y. Li, A. Krogh, R.F. Rivera-Bustamante, L. Herrera-Estrella, Y. Yin, J. Yu, K. Hu, and Z. Zhang, 2014: Whole-genome sequencing of cultivated and wild peppers provides insights into Capsicum domestication and specialization. Proceedings of the National Academy of Sciences 111, 5135-5140.

R Core Team, 2018: R: a language and environment for statistical computing, Vienna, Austria.

Ramachandran, S., J. Funderburk, J. Stavisky, and S. Olson, 2001: Population abundance and movement of Frankliniella species and Orius insidiosus in field pepper. Agricultural and Forest Entomology 3, 129-137.

Reitz, S.R., E.L. Yearby, J.E. Funderburk, J. Stavisky, M.T. Momol, and S.M. Olson, 2003a: Integrated management tactics for Frankliniella thrips (Thysanoptera: Thripidae) in fieldgrown pepper. Journal of Economic Entomology 96, 1201-1214.

Reymond, P., N. Bodenhausen, R.M.P. Van Poecke, V. Krishnamurthy, M. Dicke, and E.E. Farmer, 2004: A conserved transcript pattern in response to a specialist and a generalist herbivore. The Plant Cell 16, 3132-3147.

Richman, A., A. Swanson, T. Humphrey, R. Chapman, B. McGarvey, R. Pocs, and J. Brandle, 2005: Functional genomics uncovers three glucosyltransferases involved in the synthesis of the major sweet glucosides of Stevia rebaudiana. The Plant Journal 41, 56-67.

Rieu, I., O. Ruiz-Rivero, N. Fernandez-Garcia, J. Griffiths, S.J. Powers, F. Gong, T. Linhartova, S. Eriksson, O. Nilsson, S.G. Thomas, A.L. Phillips, and P. Hedden, 2008: The gibberellin biosynthetic genes AtGA200x1 and AtGA200x2 act, partially redundantly, to promote growth and development throughout the Arabidopsis life cycle. The Plant Journal 53, 488-504. 
Riley, D.G., S.V. Joseph, R. Srinivasan, and S. Diffie, 2011: Thrips vectors of tospoviruses. Journal of Integrated Pest Management 2, I1-I10.

Ro, D.-K., G.-I. Arimura, S.Y.W. Lau, E. Piers, and J. Bohlmann, 2005: Loblolly pine abietadienol/abietadienal oxidase PtAO (CYP720B1) is a multifunctional, multisubstrate cytochrome P450 monooxygenase. Proceedings of the National Academy of Sciences of the United States of America 102, 8060-8065.

Robinson, J.T., H. Thorvaldsdóttir, W. Winckler, M. Guttman, E.S. Lander, G. Getz, and J.P. Mesirov, 2011: Integrative genomics viewer. Nature Biotechnology 29, 24.

Ronde, D.d., D. Lohuis, and R. Kormelink, 2019: Identification and characterization of a new class of tomato spotted wilt virus isolates that break $T s w$-based resistance in a temperaturedependent manner. Plant Pathology 68, 60-71.

Rosenheim, J.A., S.C. Welter, M.W. Johnson, R.F.L. Mau, and L.R. Gusukuma-Minuto, 1990: Direct feeding damage on cucumber by mixed-species infestations of Thrips palmi and Frankliniella occidentalis (Thysanoptera: Thripidae). Journal of Economic Entomology 83, 1519-1525.

Rosenthal, J.P., and R. Dirzo, 1997: Effects of life history, domestication and agronomic selection on plant defence against insects: evidence from maizes and wild relatives. Evolutionary Ecology 11, 337-355.

Salamon, P., and J. Szabó, 2016: Symptoms caused by tomato spotted wilt virus (TSWV) in pepper (Capsicum spp.) and marker assisted selection of TSWV resistant pepper lines for hybrid constructions.

Sanatombi, K., and G.J. Sharma, 2008: In vitro plant regeneration in six cultivars of Capsicum spp. using different explants. Biologia Plantarum 52, 141-145.

Sanchez, J.A., and A. Lacasa, 2002: Modelling population dynamics of Orius laevigatus and O. albidipennis (Hemiptera: Anthocoridae) to optimize their use as biological control agents of Frankliniella occidentalis (Thysanoptera: Thripidae). Bulletin of Entomological Research 92, 77-88.

Sanford, J.C., T.M. Klein, E.D. Wolf, and N. Allen, 1987: Delivery of substances into cells and tissues using a particle bombardment process. Particulate Science and Technology 5, 27-37.

Sarde, S.J., 2019: Dynamics of transcriptional responses of plants to thrips feeding. PhD thesis, Wageningen University.

Sarde, S.J., K. Bouwmeester, J. Venegas-Molina, A. David, W. Boland, and M. Dicke, 2019: Involvement of sweet pepper CaLOX2 in jasmonate-dependent induced defence against Western flower thrips. Journal of Integrative Plant Biology 61, 1085-1098.

Schaub, G.A., 2006: Parasitogenic alterations of vector behaviour. International Journal of Medical Microbiology 296, 37-40.

Schie, C.C.N.v., and F.L.W. Takken, 2014: Susceptibility genes 101: how to be a good host. Annual Review of Phytopathology 52, 551-581. 
Schneider, C.A., W.S. Rasband, and K.W. Eliceiri, 2012: NIH Image to ImageJ: 25 years of image analysis. 9, 671.

Schopfer, C.R., and J. Ebel, 1998: Identification of elicitor-induced cytochrome P450s of soybean (Glycine max L.) using differential display of mRNA. Molecular and General Genetics 258, 315-322.

Semagn, K., R. Babu, S. Hearne, and M. Olsen, 2014: Single nucleotide polymorphism genotyping using Kompetitive Allele Specific PCR (KASP): overview of the technology and its application in crop improvement. Molecular Breeding 33, 1-14.

Shapiro, E., T. Biezuner, and S. Linnarsson, 2013: Single-cell sequencing-based technologies will revolutionize whole-organism science. Nature Reviews Genetics 14, 618.

Shipp, J.L., X. Hao, A.P. Papadopoulos, and M.R. Binns, 1998a: Impact of western flower thrips (Thysanoptera: Thripidae) on growth, photosynthesis and productivity of greenhouse sweet pepper. Scientia Horticulturae 72, 87-102.

Shipp, J.L., M.R. Binns, X. Hao, and K. Wang, 1998b: Economic injury levels for western flower thrips (Thysanoptera: Thripidae) on greenhouse sweet pepper. Journal of Economic Entomology 91, 671-677.

Siemonsma, J., and P. Kasem, 1994: Vegetables; plant resources of the South east Asia (PROSEA). Journal of Ethanopharmacology 46, 130-156.

Smith, C.M., 1989: Plant resistance to insects, 286. Wiley, New York.

Smith, C.M., 2005: Plant resistance to arthropods : molecular and conventional approaches Springer, Dordrecht, the Netherlands :.

Sobda, G., Boukar, O., Tongoona, P.B., Ayertey, J. \& Offei, K.S., 2017: Quantitative trait loci (QTL) for cowpea resistance to flower bud thrips (Megalurothrips sjostedti Trybom). International Journal of Plant Breeding and Genetics 4, 292-299.

Stafford, C.A., G.P. Walker, and D.E. Ullman, 2011: Infection with a plant virus modifies vector feeding behavior. Proceedings of the National Academy of Sciences 108, 9350-9355.

Stotz, H.U., B.R. Pittendrigh, J. Kroymann, K. Weniger, J. Fritsche, A. Bauke, and T. MitchellOlds, 2000: Induced plant defense responses against chewing insects. Ethylene signaling reduces resistance of Arabidopsis against egyptian cotton worm but not diamondback moth. Plant Physiology 124, 1007-1018.

Subhash, K., P. Venkataiah, and P. Bhaskar, 1997: EMS-induced lincomycin resistance in red pepper (Capsicum annuum L.). In Vitro Cellular \& Developmental Biology - Plant 33, 285-287.

Sun, M., R.E. Voorrips, G. Steenhuis-Broers, W. van't Westende, and B. Vosman, 2018: Reduced phloem uptake of Myzus persicae on an aphid resistant pepper accession. BMC Plant Biology 18, 138.

Sun, M., R.E. Voorrips, and B. Vosman, 2020: Aphid populations showing differential levels of virulence on Capsicum accessions. Insect Science 27, 336-348 
Takahashi, S., and T. Koyama, 2006: Structure and function of cis-prenyl chain elongating enzymes. The Chemical Record 6, 194-205.

Tang, F., C. Barbacioru, Y. Wang, E. Nordman, C. Lee, N. Xu, X. Wang, J. Bodeau, B.B. Tuch, A. Siddiqui, K. Lao, and M.A. Surani, 2009: mRNA-Seq whole-transcriptome analysis of a single cell. Nature Methods 6, 377-382.

Tang, J., B. Vosman, R.E. Voorrips, C.G. van der Linden, and J.A. Leunissen, 2006: QualitySNP: a pipeline for detecting single nucleotide polymorphisms and insertions/deletions in EST data from diploid and polyploid species. BMC Bioinformatics 7, 438.

Tang, L.D., H.Y. Zhao, B.L. Fu, Y. Han, K. Liu, and J.H. Wu, 2016: Colored sticky traps to selectively survey thrips in cowpea ecosystem. Neotropical Entomology 45, 96-101.

Teulon, D.A.J., D.R. Penman, and P.M.J. Ramakers, 1993: Volatile chemicals for thrips (Thysanoptera: Thripidae) host finding and applications for thrips pest management. Journal of Economic Entomology 86, 1405-1415.

Thornton, L.E., S.G. Rupasinghe, H. Peng, M.A. Schuler, and M.M. Neff, 2010: Arabidopsis CYP72C1 is an atypical cytochrome P450 that inactivates brassinosteroids. Plant Molecular Biology 74, 167-181.

Tikunov, Y.M., S. Laptenok, R.D. Hall, A. Bovy, and R.C.H. de Vos, 2012: MSClust: a tool for unsupervised mass spectra extraction of chromatography-mass spectrometry ion-wise aligned data. Metabolomics : Official journal of the Metabolomic Society 8, 714-718.

Tommasini, M., and S. Maini, 1995: Frankliniella occidentalis and other thrips harmful to vegetable and ornamental crops in Europe. Wageningen Agricultural University Papers.

Tommasini, M., and S. Maini, 2002: Thrips control on protected sweet pepper crops: enhancement by means of Orius laevigatus releases Thrips and Tospoviruses. Proceeding 7th International Symposium on Thysanoptera, 249-256.

Tommasini, M.G., J.C.v. Lenteren, and G. Burgio, 2004: Biological traits and predation capacity of four Orius species on two prey species. Bulletin of Insectology 57, 79-93.

Tsao, R., C.H. Marvin, A.B. Broadbent, M. Friesen, W.R. Allen, and B.D. McGarvey, 2005: Evidence for an isobutylamide associated with host-plant resistance to western flower thrips, Frankliniella occidentalis, in chrysanthemum. Journal of Chemical Ecology 31, 103-110.

Twell, D., T.M. Klein, and S. McCormick, 1997: Transformation of pollen by particle bombardment, In: K. Lindsey, (ed.) Plant Tissue Culture Manual: Supplement 7, 631-644. Springer Netherlands, Dordrecht.

Ullman, D.E., R. Meideros, L.R. Campbell, A.E. Whitfield, J.L. Sherwood, and T.L. German, 2002: Thrips as vectors of tospoviruses. Advances in Botanical Research 36, 113-140.

Untergasser, A., H. Nijveen, X. Rao, T. Bisseling, R. Geurts, and J.A.M. Leunissen, 2007: Primer3Plus, an enhanced web interface to Primer3. Nucleic Acids Research 35: W71-W74. 
Valadez-Bustos, M.G., G.A. Aguado-Santacruz, G. Carrillo-Castañeda, V.H. Aguilar-Rincón, E. Espitia-Rangel, S. Montes-Hernández, and A. Robledo-Paz, 2009: In vitro propagation and agronomic performance of regenerated chili pepper (Capsicum spp.) plants from commercially important genotypes. In Vitro Cellular \& Developmental Biology - Plant 45, 650.

Valera-Montero, L.L., and N. Ochoa-Alejo, 1992: A novel approach for chili pepper (Capsicum annuum L.) plant regeneration: shoot induction in rooted hypocotyls. Plant Science 84, 215-219.

Van den Oever-Van den Elsen, F., A.F. Lucatti, S. Van Heusden, C. Broekgaarden, R. Mumm, M. Dicke, and B. Vosman, 2016: Quantitative resistance against Bemisia tabaci in Solanum pennellii: genetics and metabolomics. Journal of Integrative Plant Biology 58, 397-412.

Van Der Vossen, E., A. Sikkema, B.t.L. Hekkert, J. Gros, P. Stevens, M. Muskens, D. Wouters, A. Pereira, W. Stiekema, and S. Allefs, 2003: An ancient R gene from the wild potato species Solanum bulbocastanum confers broad-spectrum resistance to Phytophthora infestans in cultivated potato and tomato. The Plant Journal 36, 867-882.

Van Haperen, P., R.E. Voorrips, J.J.A. van Loon, and B. Vosman, 2019: The effect of plant development on thrips resistance in Capsicum. Arthropod-Plant Interactions 13, 11-18.

Van Lenteren, J.C., and A.J. Loomans, 1999: Biological control of thrips: how far are we. Bulletin IOBC 22, 141-44.

Van Rijn, P.C., C. Mollema, and G.M. Steenhuis-Broers, 1995: Comparative life history studies of Frankliniella occidentalis and Thrips tabaci (Thysanoptera: Thripidae) on cucumber. Bulletin of Entomological Research 85, 285-297.

Varet, H., L. Brillet-Guéguen, J.-Y. Coppée, and M.-A. Dillies, 2016: SARTools: a DESeq2and EdgeR-Based R Pipeline for Comprehensive Differential Analysis of RNA-Seq Data. PLoS ONE 11, e0157022.

Villarroel, C.A., W. Jonckheere, J.M. Alba, J.J. Glas, W. Dermauw, M.A. Haring, T. Van Leeuwen, R.C. Schuurink, and M.R. Kant, 2016: Salivary proteins of spider mites suppress defenses in Nicotiana benthamiana and promote mite reproduction. The Plant Journal 86, 119-131.

Visschers, I.G.S., J.L. Peters, J.A.H. van de Vondervoort, R.H.M. Hoogveld, and N.M. van Dam, 2019a: Thrips resistance screening is coming of age: Leaf position and ontogeny are important determinants of leaf-based resistance in pepper. Frontiers in Plant Science $\mathbf{1 0 .}$

Visschers, I.G.S., J.L. Peters, L.L.H. Timmermans, E. Edwards, J.B. Ferrater, C.H. Balatero, M. Stratongjun, P.M. Bleeker, Z. van Herwijnen, G.A. Glawe, J. Bruin, N.M. van Dam, and M. Macel, 2019b: Resistance to three thrips species in Capsicum spp. depends on site conditions and geographic regions. Journal of Applied Entomology.

Voorrips, R.E., R. Finkers, L. Sanjaya, and R. Groenwold, 2004: QTL mapping of anthracnose (Colletotrichum spp.) resistance in a cross between Capsicum annuum and $C$. chinense. Theoretical and Applied Genetics 109, 1275-1282. 
Voorrips, R.E., G. Steenhuis-Broers, M. Tiemens-Hulscher, and E.T. Lammerts van Bueren, 2008: Plant traits associated with resistance to Thrips tabaci in cabbage (Brassica oleracea var capitata). Euphytica 163, 409.

Vosman, B., W.P.C. van't Westende, B. Henken, H.D.L.M. van Eekelen, R.C.H. de Vos, and R.E. Voorrips, 2018: Broad spectrum insect resistance and metabolites in close relatives of the cultivated tomato. Euphytica 214, 46.

Vosman, B., A. Kashaninia, W. van't Westende, F. Meijer-Dekens, H. van Eekelen, R.G.F. Visser, R.C.H. de Vos, and R.E. Voorrips, 2019: QTL mapping of insect resistance components of Solanum galapagense. Theoretical and Applied Genetics 132, 531-541.

VSN International, 2015: Genstat for Windows 18th Edition. VSN International, Hemel Hempstead, UK.

Walker, K., R. Long, and R. Croteau, 2002: The final acylation step in Taxol biosynthesis: cloning of the taxoid $\mathrm{C} 13$-side-chain $\mathrm{N}$-benzoyltransferase from Taxus. Proceedings of the National Academy of Sciences 99, 9166-9171.

Wang, J.C., B. Zhang, H.G. Li, J.P. Wang, and C.Y. Zheng, 2014: Effects of exposure to high temperature on Frankliniella occidentalis (Thysanoptera: Thripidae), under arrhenotoky and sexual reproduction conditions. Florida entomologist 97, 504-511.

Weintraub, P.G., 2007: Integrated control of pests in tropical and subtropical sweet pepper production. Pest Management Science 63, 753-760.

Welter, S.C., J.A. Rosenheim, M.W. Johnson, R.F.L. Mau, and L.R. Gusukuma-Minuto, 1990: Effects of Thrips palmi and western flower thrips (Thysanoptera: Thripidae) on the yield, growth, and carbon allocation pattern in cucumbers. Journal of Economic Entomology 83, 2092-2101.

Whitfield, A.E., D.E. Ullman, and T.L. German, 2005: Tospovirus-thrips interactions. Annual Review of Phytopathology 43, 459-489.

Wijkamp, I., N. Almarza, R. Goldbach, and D. Peters, 1995: Distinct levels of specificity in thrips transmission of tospoviruses. Phytopathology 85, 1069-1074.

Williams, J., A.L. Phillips, P. Gaskin, and P. Hedden, 1998: Function and substrate specificity of the gibberellin 3 $\beta$-hydroxylase encoded by the Arabidopsis GA4 Gene. Plant Physiology 117, 559-563.

Yalovsky, S., C.E. Trueblood, K.L. Callan, J.O. Narita, S.M. Jenkins, J. Rine, and W. Gruissem, 1997: Plant farnesyltransferase can restore yeast Ras signaling and mating. Molecular and Cellular Biology 17, 1986-1994.

Yi, G., J.M. Lee, S. Lee, D. Choi, and B.-D. Kim, 2006: Exploitation of pepper EST-SSRs and an SSR-based linkage map. Theoretical and Applied Genetics 114, 113-130.

Zhang, Z.J., Q.J. Wu, X.F. Li, Y.J. Zhang, B.Y. Xu, and G.R. Zhu, 2007: Life history of western flower thrips, Frankliniella occidentalis (Thysan., Thripae), on five different vegetable leaves. Journal of Applied Entomology 131, 347-354. 
Zhao, X., Z. Meng, Y. Wang, W. Chen, C. Sun, B. Cui, J. Cui, M. Yu, Z. Zeng, S. Guo, D. Luo, J.Q. Cheng, R. Zhang, and H. Cui, 2017: Pollen magnetofection for genetic modification with magnetic nanoparticles as gene carriers. Nature Plants 3, 956-964.

Zi, J., S. Mafu, and R.J. Peters, 2014: To gibberellins and beyond! Surveying the evolution of (di)terpenoid metabolism. Annual Review of Plant Biology 65, 259-286.

Znidarcic, D., N. Valic, and S. Trdan, 2008: Epicuticular wax content in the leaves of cabbage (Brassica oleracea L. var. capitata) as a mechanical barrier against three insect pests. Acta Agriculturae Slovenica 91, 361. 


\section{Acknowledgement}

The past 4.5 years have been an important chapter in my life. Although, like every $\mathrm{PhD}$, I have had ups and downs during this journey, I never regretted the moment I decided to accept this challenge. I could not have done this without a few people that I would like to thank in this most read part of the thesis (except, maybe, the separate sheet in the front of this book).

First of all, I would like to thank my promotor, Joop, and my co-promotors, Ben and Roeland. I am very grateful that I got the opportunity to start this project, and your trust that together we would make it happen! Joop, thank you for your contributions to our meetings, and especially for keeping the insect-side of the story in mind as well. Your knowledge of everything related to plant-insect interactions, and many other topics, is admirable. Ben, thank you for your input in the project as well. I learned a lot from you on how to design the experiments and how to use the potential of the plant material to the fullest. Even during your holidays, you were always eager to know whether I got interesting results. Roeland, I would also like to thank you for your input in our meetings. Especially your expertise in experimental design and statistics helped to make this part of the thesis less complicated for me.

I would like to thank Nunhems Netherlands BV and Bejo Zaden BV for their contributions, in particular Alejandro and Wijnand. Alejandro, thank you for convincing me that I should do a PhD during one of our many car rides, and thank you for your helpful remarks during the committee meetings. Wijnand, thank you for helping me with the DNA isolation and marker analysis, your high throughput methods saved me literally a few weeks time. Also your contribution during the consortium meetings was highly appreciated. Also thanks to Nelson and Henk, who also contributed to the project.

Many thanks go to Betty and Wendy, the technicians who supported me a lot during the big experiments. Betty, you learned me how to deal with these little tiny creatures. Sometimes it really makes you wonder why these pests are actually a problem, seeing how easy it is to kill them with a tiny push of your almost hairless brush. Thank you for your help over the last years, and thank you for the nice conversations and coffee breaks! Wendy, thank you for helping me during the big experiments, when even Betty and I could not handle all things that needed to be done. I always liked to work together, finding the most optimal way to complete all the tasks, and also the nice conversations that we had about anything. Without the two of you, this thesis would not have been here! 
I would also like to thank my dear colleague and fellow PhD-er, Mengjing. Starting almost at the same time, it was nice to have a friend that knows exactly what you're going through! I always enjoyed talking about science to you, you always had nice ideas on how to tackle problems and what would be interesting to do next. I am glad we started, and now finished our PhDs together, literally side by side.

Furthermore, I would like to emphasize the importance of having lunch. During $\mathrm{PhD}$, I considered lunch as the most important meal of the day, not only because this was the only way to silence my belly, but also because of the amazing people and their even more amazing stories that we shared during the lunch break. I would like to especially thank the most dedicated lunch-buddies Mas, Jasper, Charlotte, Jeroen and Johan. Mas, your laugh always warned me that it was time to have a break, even though the secretary of the other department did not agree. Thank you for being there, enlightening my day, and thank you for being my paranymph! Jasper, thank you for sharing your stories, and thank you for being my office mate! Charlotte, every time Jarst told a joke, I was usually laughing more about you trying not to laugh than about the joke itself. Jeroen and Johan, your stories always made me laugh, and this way you really helped me to reset my brain for the last part of the day!

Also many thanks to my other WUR-colleagues. Nicole, Daniëlle and Letty, thank you for taking care of many, many things, your support is very much appreciated. Martijn, thank you for helping me with the RNA-seq data. Thanks to all colleagues at plant breeding for showing your interest in my project, for asking how I was doing, and for giving valuable feedback during the presentations. Especially thanks to Aina, Anne, Behzad, Bettina, Carolina, Cynara, Eleni, Geert, Johan, Kaile, Mathilde, Michiel, Miguel, Peter, Peter, Sri, Viviana, Xiao, Xuexue and Yan. Also many thanks to Gurnoor and Jaspinder, who invited me to meet their family and friends during one of the most impressive trips in my life. Many thanks to the hardworking people at Unifarm, especially Sean, who were taking excellent care of my plants. I would also like to thank Ric and Henriëtte for their help with the metabolomics data analysis. Also thanks to Gerrie and Sandeep, for sharing your experiences with thrips. It was always nice to know there was a back-up thrips rearing in case I needed more!

Also, I would like to thank my new colleagues for their support. Thanks René for giving me the opportunity to take some days off to finish the thesis. Also thanks to the other KeyGeners for the warm welcome and your interest in my project. I would also like to thank my other colleagues from the Bongerd, especially Tijmen, for your interest in how I was dealing with the PhD. 
Of course there is many people outside work atmosphere that also supported me along the past few years. I would like to thank Jaap, Matthijs and Susanne for escaping so many times. Furthermore, thanks to my third-Monday-of-the-month friends, Sabine, Rosaline and Ruben. Although our average beer-intake has significantly decreased over the last few years, probably correlated to the increase of cars and traveling distance, I still enjoy those moments we share! Also thanks to all the board gamers, Jaap, Kim, Nicole, Suraj, Viet. Special thanks to Lisette, Carolien, Marieke and Remke, for the nice trips we had and your successful attempts to make me relax during the writing phase. Thanks to my other fellow (former) badminton players, from BC Barneveld and from BC de Lobbers, especially to Annemieke, Jochem, Julia, Niels, Ollie and Stephanie. Thanks to my fellow Bioloogjes, Christel, Dylan, Esther, Hetty, Janna, Jeanine, Joyce, Margriet and Ramona.

Natuurlijk heb ik ook veel steun gehad aan mijn familie. Schoonmoeder Kune, bedankt voor je interesse in mijn project, en de leuke gesprekken. Bart en Mylène, ook jullie hebben me veel gesteund, en de leuke filmpjes en foto's van de nieuwste fratsen van mijn favoriete neefje en nichtje waren zeer welkom. Oma en opa, als ik weer eens in de buurt was, waren jullie altijd benieuwd naar mijn onderzoek en wanneer dat boekje nou eens klaar was. Bedankt voor de goede zorgen! Renée, ook al was ik afgelopen periode niet de meest gezellige zus, toch zijn we er zonder al te veel kleerscheuren doorheen gekomen. Misschien wel door de noeste nacho's? Ik beloof dat ik de komende periode weer een leukere zus ga zijn! Papa en mama, ook jullie hebben me enorm gesteund in de afgelopen periode, ook altijd geïnteresseerd in mijn onderzoek, mijn plannen, hoe het met schrijven ging, in alles! Bedankt voor jullie steun, niet alleen de afgelopen jaren, maar ook alle jaren daarvoor. Zonder jullie was ik niet de persoon die ik nu ben.

Lieve Jarst, ik ben enorm dankbaar en blij dat onze PhD's en onze liefde voor muziek ons bij elkaar hebben gebracht. Je maakt me elke dag weer aan het lachen. Bedankt voor je steun, liefde en goede zorgen. Deze reis was een stuk minder leuk geweest zonder jou. Blijf wie je bent, en op naar een nieuw avontuur! 


\section{About the author}

Pauline van Haperen (17 October, 1990) was born in Breda and raised nearby in Prinsenbeek in Noord-Brabant, where she also spent her childhood. She initially started a sports career in gymnastics and received every gold medal that could be won. Her success continued until the referees decided that gymnastics involved more than just putting on a nice show. Nevertheless, she managed to continue her success by making an early career switch to badminton. Besides her talent in badminton and a (healthy) competitive spirit, she was also very good at doing the exact opposite of what people told her to do. Only after she had received her Gymnasium diploma and had left Prinsenbeek to study Biology in

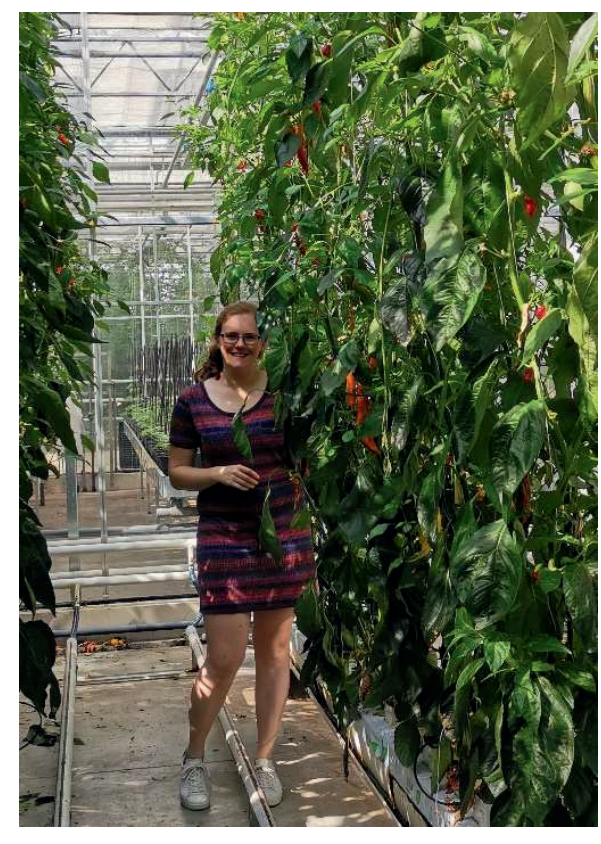
Wageningen, she decided to let her hair grow longer again, because there was no point if there was nobody to rebel against anymore.

It was the year 2008 that started a new chapter in Pauline's life: a new city, a new study, longer hair, and of course more badminton. She switched to badminton club BC De Lobbers, of which its name is derived from the lob shot. Although she despises this technique, she has trained at De Lobbers for many years. She became one of the best players around and was also an active (board) member of the association. She took additional trainings and played competition in Barneveld and this way improved her badminton skills even further, in order to take part of the Second Division in the Netherlands. Nowadays, she is a trainer/coach of the advanced group of competition players of BC De Lobbers.

Soon after finishing her MSc theses called 'Role of auxin in root organ development in Parasponia andersonii' and 'The effects of long-term mild heat stress on male gametes in tomato' and receiving her MSc degree in biology in 2014, Pauline had a temporary position at Nunhems Zaden in Limburg. She worked as a research assistant in marker-assisted breeding in leafy vegetables to already get a grasp of what it is like to work at a breeding company. Regardless of the distance she had to travel between Wageningen and Nunhems every day, she came to the conclusion that she wanted to be more involved in research projects. Therefore, 
before applying for a permanent position in a breeding company, she was determined to do a PhD first. And so it happened.

In 2015 Pauline started her PhD project at Plant Breeding and Entomology about 'Towards breeding thrips-resistant varieties in Capsicum' under the supervision of prof.dr. Joop J.A. van Loon, dr. Ben Vosman and dr. Roeland E. Voorrips. Throughout her PhD, Pauline learned a lot about interactions between thrips and plants and managing long-term projects in general. Further, no matter what she was working on during the day, she always enjoyed the many lunch breaks together with her fellow colleagues. This way, she would not have to think about work only, but she could also talk about a lot of interesting nonsense topics. Also outside the department she enjoyed her PhD, especially a conference in Berlin in 2018 which she attended together with her close colleague and friend Mengjing Sun.

She is currently working at KeyGene N.V. in Wageningen as a researcher on, amongst others, banana. Yes, you have read it correctly. Banana. She personally hates everything about this delicious curved and yellow fruit, but she is still very eager to research this crop in the name of science. This is the kind of sacrifice for science that one can expect from someone with a PhD.

Nevertheless, Pauline's thesis is not about banana, but about thrips resistance in pepper. Hopefully you have enjoyed reading her exciting and useful research towards improved pepper cultivars!

Written by Jarst van Belle 


\section{Educational statement}

\section{Education Statement of the Graduate School \\ Experimental Plant Sciences}

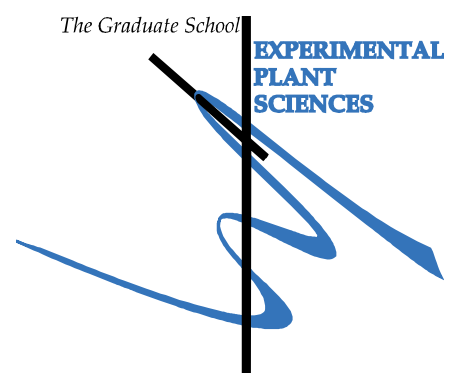

Issued to: Pauline van Haperen

Date: 24 March 2020

Group: $\quad$ Plant Breeding \& Entomology

University: Wageningen University \& Research

\section{$\underline{\text { Date }}$}

- First presentation of your project

Thrips resistance in Capsicum - Proposal

21 Jan 2016

\section{Subtotal Start-Up Phase}

1.5 credits*

\section{2) Scientific Exposure}

\section{- EPS PhD student days}

EPS PhD student days "Get2Gether", Soest

EPS PhD student days "Get2Gether", Soest

EPS PhD student days "Get2Gether", Soest

\section{- EPS theme symposia}

EPS Theme 2 symposium \& Willie Commelin Scholten Day, Leiden

EPS Theme 2 symposium \& Willie Commelin Scholten Day, Wageningen

EPS Theme 3 symposium, Wageningen

EPS Theme 1 symposium, Wageningen

EPS Theme 3 symposium, Wageningen

EPS Theme 2 symposium \& Willie Commelin Scholten Day, Wageningen

\section{- Lunteren Days and other national platforms}

Annual meeting 'Experimental Plant Sciences', Lunteren

Annual meeting 'Experimental Plant Sciences', Lunteren

Annual meeting 'Experimental Plant Sciences', Lunteren

Networking Event TKI Horticulture \& Propagation Materials, Nieuwegein

\section{Date}

Jan 28-29, 2016

Feb 09, 2017

Feb 15-16, 2018

$\operatorname{Jan} 22,2016$

Jan 23, 2017

Mar 14, 2017

Jan 30, 2018

Mar 13, 2018

Feb 01, 2019

Apr 11-12, 2016

Apr 10-11, 2017

Apr 09-10 2019

Apr 03, 2018 
Start-up Consortium meeting " Thrips resistance in pepper"

Consortium meeting "Thrips resistance in pepper"

Consortium meeting "Thrips resistance in pepper"

Consortium meeting "Thrips resistance in pepper"

Consortium meeting "Thrips resistance in pepper"

Consortium meeting "Thrips resistance in pepper"

Consortium meeting "Thrips resistance in pepper"

Consortium meeting "Thrips resistance in pepper"
Sep 14, 2015

Mar 14, 2016

Oct 14, 2016

May 09, 2017

Nov 07, 2017

Jul 02, 2018

Nov 05, 2018

Jun 24, 2019

Sep 29, 2015

Dec 15, 2016

Mar 08, 2018

Oct 16, 2018

Feb 08, 2017

Dec 08, 2015

$\operatorname{Jan} 21,2016$

Mar 16, 2016

May 23, 2016

Nov 16, 2016

Dec 07, 2017

Apr 25, 2019

May 23, 2019

Aug 29, 2019

\section{- Seminar plus}

\section{- International symposia and congresses}

International CRC 973 Symposium 'Bridging Ecology and Molecular Biology: Organic responses to recurring stress', Berlin, Germany

Apr 09-11, 2018

10th European Plant Science Retreat, Utrecht

Jul 03-06, 2018 
- Presentations

Presentation at Consortium meeting - Proposal

Mar 14, 2016

Presentation at Consortium meeting - End presentation

June 24, 2019

Presentation EPS Theme 2 Symposium \& Willie Commelin Scholten Day

Feb 01, 2019

Presentation CEPLAS Transatlantic Summer School

May 28, 2019

Presentation SLU-WUR Plant Breeding and Biotechnology

Jun 12, 2019

Poster "Thrips resistance in Capsicum changes during plant development" Network Event TKI Horticulture \& Propagation Materials

Apr 03, 2018

Poster "The effect of plant development on thrips resistance in Capsicum" 10th European Plant Science Retreat

Jul 03-06, 2018

Poster "The effect of three QTLs and two backgrounds on thrips resistance in Capsicum" Annual Meeting Experimental Plant Sciences

Apr 09, 2019

- IAB interview

- Excursions

Company visit KeyGene

Oct 12, 2017

Company visit Koppert

Oct 26, 2018

Subtotal Scientific Exposure

18.5 credits*

\section{3) In-Depth Studies}

\section{Date}

\section{- Advanced scientific courses \& workshops}

Data analysis and visualizations in $\mathrm{R}$

May $11-12,2017$

Introduction to $\mathrm{R}$ for statistical analysis

May $17-18,2018$

The power of RNAseq

Jun $11-13,2018$

CEPLAS Transatlantic Summer School Frontiers in Plant Sciences

May 27-31, 2019

SLU-WUR Plant Breeding and Biotechnology

Jun 11-13, 2019

\section{- Journal club}

\section{- Individual research training}


4) Personal Development

\section{- General skill training courses}

EPS introduction course

PhD Competence assessment

Project and time management

Wageningen Graduate Schools (WGS) PhD Workshop

Carousel

Scientific writing

\section{$\underline{\text { Date }}$}

Feb 11, 2016

Nov 10, 2016

Jan-Mar 2017

Apr 07, 2017

May-Jul, 2017

\section{Organisation of meetings, PhD courses or outreach}

\section{- activities}

Chairing and organisation of group meetings from PBR

Insect and Nonhost Resistance Group

Aug-Jul, 2016-2017

Chairing and organisation of group meetings from PBR

Insect and Nonhost Resistance Group

Aug-Jul, 2017-2018

Chairing and organisation of group meetings from PBR

Insect and Nonhost Resistance Group

Aug-Jul, 2018-2019

- Membership of EPS PhD Council

\section{TOTAL NUMBER OF CREDIT POINTS*}

32.4

Herewith the Graduate School declares that the PhD candidate has complied with the educational requirements set by the Educational Committee of EPS with a minimum total of 30 ECTS credits.

* A credit represents a normative study load of 28 hours of study. 
The research described in this thesis was financially supported by a grant (TKI 1409-045) from the Ministry of Economic Affairs of the Netherlands and the breeding companies Nunhems Netherlands B.V. and Bejo Zaden B.V.

Financial support from Wageningen University \& Research for printing this thesis is gratefully acknowledged.

Cover design by Renée van Haperen

Printed by ProefschriftMaken | Digiforce 
